\title{
Exploiting a Difference in Leaving Group Ability: An Approach to $\beta$-Substituted Monofluoroalkenes Using gem-Chlorofluoropropenes
}

\author{
Jean-Denys Hamel, Mélissa Cloutier and Jean-François Paquin* \\ CCVC, PROTEO, Département de chimie, 1045 avenue de la Médecine, \\ Université Laval, Québec, Québec, Canada, GIV 0A6. \\ E-mail: jean-francois.paquin@chm.ulaval.ca
}

\section{Table of Contents}

1. General information

2. Synthesis of 3-chloro-3-fluoropropenes

3. Synthesis of monofluoroalkenes

4. Synthetic transformations of monofluoroalkenes

5. References

6. ${ }^{1} \mathrm{H},{ }^{19} \mathrm{~F}$ and ${ }^{13} \mathrm{C}$ spectra

7. Crystal structure of $(\boldsymbol{E}) \mathbf{- 1 2 a} \cdot \mathbf{H C l}$

\section{General information}

The following includes general experimental procedures, specific details for representative reactions, and isolation and spectroscopic information for the new compounds prepared. All reactions were carried out under an argon atmosphere with dry solvents. $\mathrm{Et}_{2} \mathrm{O}, \mathrm{THF}, \mathrm{CH}_{3} \mathrm{CN}, \mathrm{CH}_{2} \mathrm{Cl}_{2}$ and toluene were purified using a Vacuum Atmospheres Inc. Solvent Purification System. All other commercially available compounds were used as received. Thin-layer chromatography (TLC) analysis of reaction mixtures was performed using Silicycle silica gel 60 A F254 TLC plates, and visualized under UV or by staining with either potassium permanganate or phosphomolybdic acid. Flash column chromatography was carried out on Silicycle silica gel $60 \AA$, 230-400 mesh. ${ }^{1} \mathrm{H},{ }^{13} \mathrm{C}$ and ${ }^{19} \mathrm{~F}$ NMR spectra were recorded in $\mathrm{CDCl}_{3}$ or DMSO- $\mathrm{d}_{6}$ at ambient temperature using Agilent DD2 500 and Varian Inova 400 spectrometers. ${ }^{1} \mathrm{H}$ and ${ }^{13} \mathrm{C}$ 
NMR chemical shifts are reported in ppm downfield of tetramethylsilane and are respectively referenced to tetramethylsilane $(\delta=0.00 \mathrm{ppm})$ and residual solvent $(\delta=$ $77.16 \mathrm{ppm}$ for $\mathrm{CDCl}_{3} ; \delta=39.52$ for $\left.\mathrm{DMSO}_{-} \mathrm{d}_{6}\right)$. For ${ }^{19} \mathrm{~F} \mathrm{NMR}, \mathrm{CFCl}_{3}$ is used as the external standard. High-resolution mass spectra were obtained on a LC/MS-TOF Agilent 6210 using electrospray ionization (ESI). Infrared spectra were recorded using a Thermo Scientific Nicolet 380 FT-IR spectrometer. Melting points were recorded on a Stanford Research System OptiMelt capillary melting point apparatus and are uncorrected.

\section{Synthesis of 3-chloro-3-fluoropropenes}

Typical procedure for the electrophilic chlorination of $\alpha$-fluoroketones ${ }^{1}$ : A solution of the $\alpha$-fluoroketone in $\mathrm{CH}_{2} \mathrm{Cl}_{2}(0.4 \mathrm{M})$ was brought to $0{ }^{\circ} \mathrm{C}$ under $\operatorname{argon} . \mathrm{Et}_{3} \mathrm{~N}$ (1.1 equiv) was added, followed by TMSOTf (1 equiv). The resulting solution was allowed to reach ambient temperature over 1 hour, and then brought back to $0{ }^{\circ} \mathrm{C}$. NCS (1.1 equiv) was added, and the mixture was allowed to reach ambient temperature over 18 hours. Water was added and the mixture was extracted with $\mathrm{Et}_{2} \mathrm{O}(3 \mathrm{x})$. The combined organic layers were washed with brine (1x), dried over $\mathrm{Na}_{2} \mathrm{SO}_{4}$, and concentrated in vacuo.

\section{Typical procedure for the Peterson olefination of $\alpha$-chloro- $\alpha$-fluoroketones: A} solution of (trimethylsilyl)methyl magnesium chloride (1.2 equiv) in $\mathrm{Et}_{2} \mathrm{O}(1.0 \mathrm{M})$ was brought to $0{ }^{\circ} \mathrm{C}$ under argon. A solution of the $\alpha$-chloro- $\alpha$-fluoroketone (1 equiv) in $\mathrm{Et}_{2} \mathrm{O}$ $(0.4 \mathrm{M})$ was then added dropwise. The resulting solution was allowed to reach ambient temperature over 2 hours. A saturated aqueous solution of ammonium chloride was added to the reaction mixture. The layers were separated and the aqueous layer was extracted with $\mathrm{CH}_{2} \mathrm{Cl}_{2}$ (2x). The combined organic layers were dried over $\mathrm{Na}_{2} \mathrm{SO}_{4}$ and concentrated in vacuo. The resulting oil was taken up in $\mathrm{CH}_{2} \mathrm{Cl}_{2}(0.2 \mathrm{M})$ and $\mathrm{BF}_{3} \cdot \mathrm{Et}_{2} \mathrm{O}$ (0.4 equiv) was added. The resulting solution was stirred at ambient temperature for 2 hours. The reaction mixture was diluted with hexanes, washed with saturated aqueous sodium bicarbonate $(1 \mathrm{x})$ and brine $(1 \mathrm{x})$, then dried over $\mathrm{MgSO}_{4}$ and concentrated in vасио. 

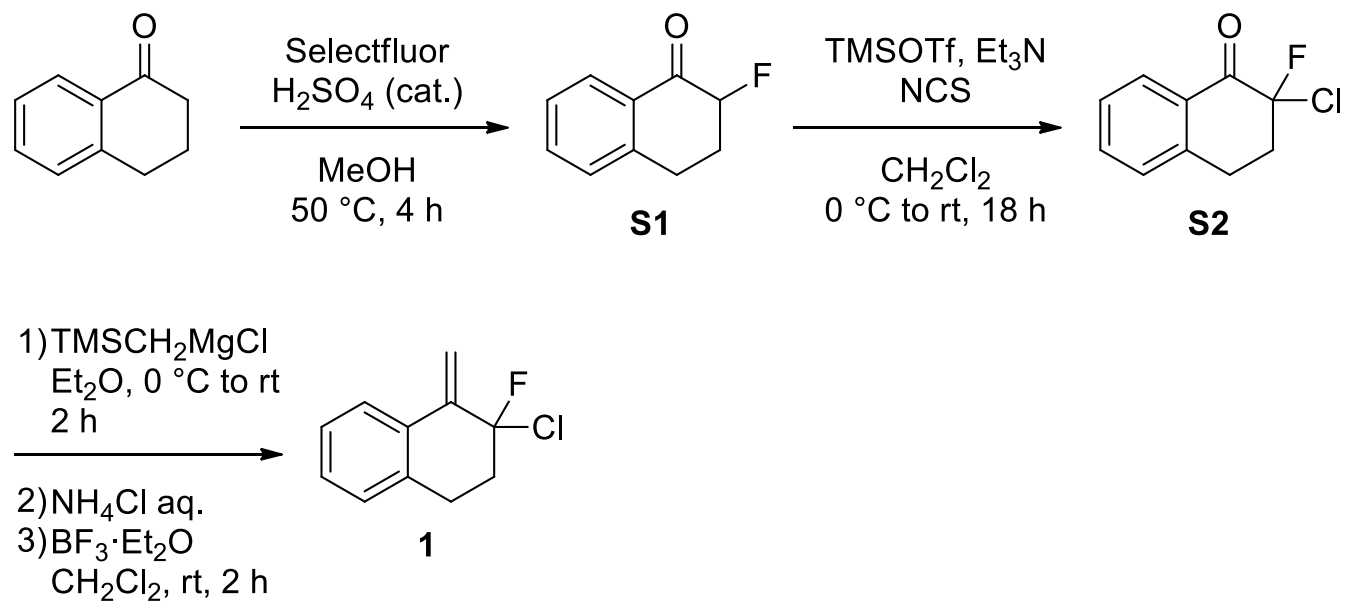

2-Fluoro-1-tetralone (S1). The $\alpha$-fluoroketone S1 was prepared from 1-tetralone following a literature procedure. ${ }^{2}$ Spectroscopic data was in agreement with the literature. $^{2}$

2-Chloro-2-fluoro-1-tetralone (S2). The $\alpha$-chloro- $\alpha$-fluoroketone $\mathbf{S 2}$ was prepared following the general procedure for the electrophilic chlorination of S1. On a $18.1 \mathrm{mmol}$ scale, the desired product (3.38 $\mathrm{g} \mathrm{mg}$, 94\%) was isolated as a colorless oil by flash chromatography using 10\% EtOAc/hexanes. Spectroscopic data was in agreement with the literature. ${ }^{1}$

2-Chloro-2-fluoro-1-methylene-1,2,3,4-tetrahydronaphthalene (1). The 3-chloro-3fluoropropene 1 was prepared following the general procedure for the Peterson olefination of S2. On a $9.20 \mathrm{mmol}$ scale, the desired product (1.33 g, 73\%) was isolated as a colorless oil by flash chromatography using hexanes. Spectroscopic data was in agreement with the literature. ${ }^{3}$ 

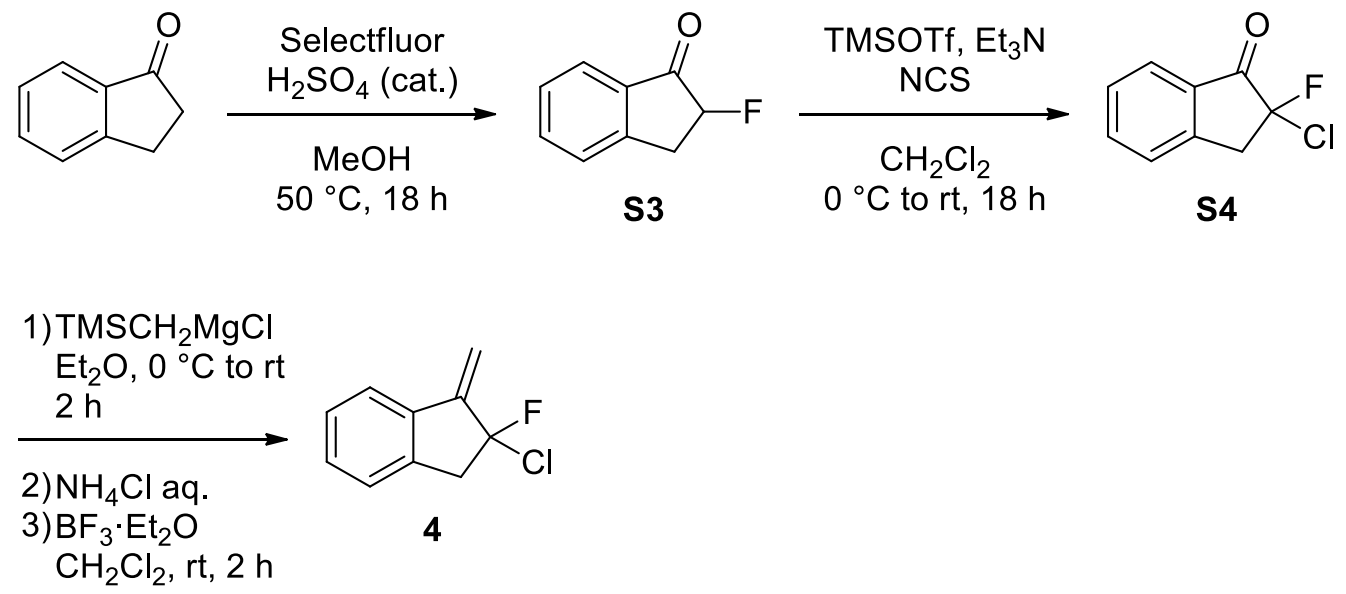

2-Fluoro-1-indanone (S3). The $\alpha$-fluoroketone S3 was prepared from 1-indanone following a literature procedure. ${ }^{2}$ Spectroscopic data was in agreement with the literature. $^{4}$

2-Chloro-2-fluoro-1-indanone (S4). The $\alpha$-chloro- $\alpha$-fluoroketone $\mathbf{S 4}$ was prepared following the general procedure for the electrophilic chlorination of S3. On a $6.96 \mathrm{mmol}$ scale, the desired product $(1.11 \mathrm{~g}, 87 \%)$ was isolated as a yellow oil by flash chromatography using 5\% EtOAc/hexanes. IR (ATR, ZnSe) $v=3078,2936,1735,1609$, 1468, 1217, 1102, 998, 918, $732 \mathrm{~cm}^{-1} ;{ }^{1} \mathrm{H}$ NMR (500 MHz, $\left.\mathrm{CDCl}_{3}\right) \delta 7.89$ (d, 1H, J = 7.6 $\mathrm{Hz}), 7.74(\mathrm{t}, 1 \mathrm{H}, J=7.5 \mathrm{~Hz}), 7.52-7.46(\mathrm{~m}, 2 \mathrm{H}), 3.90-3.79(\mathrm{~m}, 2 \mathrm{H}) ;{ }^{19} \mathrm{~F}$ NMR $(470 \mathrm{MHz}$, $\left.\mathrm{CDCl}_{3}\right) \delta-120.4(\mathrm{~m}, 1 \mathrm{~F}) ;{ }^{13} \mathrm{C} \mathrm{NMR}\left(126 \mathrm{MHz}, \mathrm{CDCl}_{3}\right) \delta 191.7\left(\mathrm{~d}, J_{\mathrm{C}-\mathrm{F}}=22.4 \mathrm{~Hz}\right), 147.1$ $\left(\mathrm{d}, J_{\mathrm{C}-\mathrm{F}}=5.5 \mathrm{~Hz}\right), 137.4,131.2\left(\mathrm{~d}, J_{\mathrm{C}-\mathrm{F}}=1.8 \mathrm{~Hz}\right), 129.2,126.7\left(\mathrm{~d}, J_{\mathrm{C}-\mathrm{F}}=1.7 \mathrm{~Hz}\right), 126.3$ $\left(\mathrm{d}, J_{\mathrm{C}-\mathrm{F}}=0.6 \mathrm{~Hz}\right), 106.1\left(\mathrm{~d}, J_{\mathrm{C}-\mathrm{F}}=262 \mathrm{~Hz}\right), 44.0\left(\mathrm{~d}, J_{\mathrm{C}-\mathrm{F}}=23.8 \mathrm{~Hz}\right)$; HRMS-ESI calcd for $\mathrm{C}_{9} \mathrm{H}_{7} \mathrm{ClFO}[\mathrm{M}+\mathrm{H}]^{+}$185.0164; found 185.0158.

\section{2-Chloro-2-fluoro-1-methylene-2,3-dihydro-1H-indene (4). The 3-chloro-3-}

fluoropropene 4 was prepared following the general procedure for the Peterson olefination of $\mathbf{S 4}$. On a $5.21 \mathrm{mmol}$ scale, the desired product $(648 \mathrm{mg}, 68 \%)$ was isolated as a colorless oil by flash chromatography using pentane. IR (ATR, ZnSe) $v=3029$, 2915, 1651, 1425, 1281, 1089, 976, 775, 727, $707 \mathrm{~cm}^{-1} ;{ }^{1} \mathrm{H}$ NMR $\left(500 \mathrm{MHz}, \mathrm{CDCl}_{3}\right) \delta$ $7.51(\mathrm{~d}, 1 \mathrm{H}, J=7.1 \mathrm{~Hz}), 7.33-7.23(\mathrm{~m}, 3 \mathrm{H}), 5.78(\mathrm{~d}, 1 \mathrm{H}, J=2.9 \mathrm{~Hz}), 5.72(\mathrm{~d}, 1 \mathrm{H}, J=2.7$ $\mathrm{Hz}), 3.74-3.72(\mathrm{~m}, 2 \mathrm{H}) ;{ }^{19} \mathrm{~F}$ NMR $\left(470 \mathrm{MHz}, \mathrm{CDCl}_{3}\right) \delta-95.7(\mathrm{~m}, 1 \mathrm{~F}) ;{ }^{13} \mathrm{C}$ NMR $(126$ $\left.\mathrm{MHz} \mathrm{CDCl}_{3}\right) \delta 149.5\left(\mathrm{~d}, J_{\mathrm{C}-\mathrm{F}}=19.3 \mathrm{~Hz}\right), 138.2\left(\mathrm{~d}, J_{\mathrm{C}-\mathrm{F}}=4.0 \mathrm{~Hz}\right), 136.1\left(\mathrm{~d}, J_{\mathrm{C}-\mathrm{F}}=2.4\right.$ $\mathrm{Hz}), 130.0,128.0,125.1\left(\mathrm{~d}, J_{\mathrm{C}-\mathrm{F}}=1.8 \mathrm{~Hz}\right), 121.5,113.8\left(\mathrm{~d}, J_{\mathrm{C}-\mathrm{F}}=253 \mathrm{~Hz}\right), 109.1\left(\mathrm{~d}, J_{\mathrm{C}-\mathrm{F}}\right.$ 
$=4.0 \mathrm{~Hz}), 48.5 \mathrm{~Hz}\left(\mathrm{~d}, J_{\mathrm{C}-\mathrm{F}}=24.1 \mathrm{~Hz}\right) ; \mathrm{HRMS}-\mathrm{ESI}$ calcd for $\mathrm{C}_{10} \mathrm{H}_{9} \mathrm{ClF}[\mathrm{M}+\mathrm{H}]^{+} 183.0371$; found 183.0381.
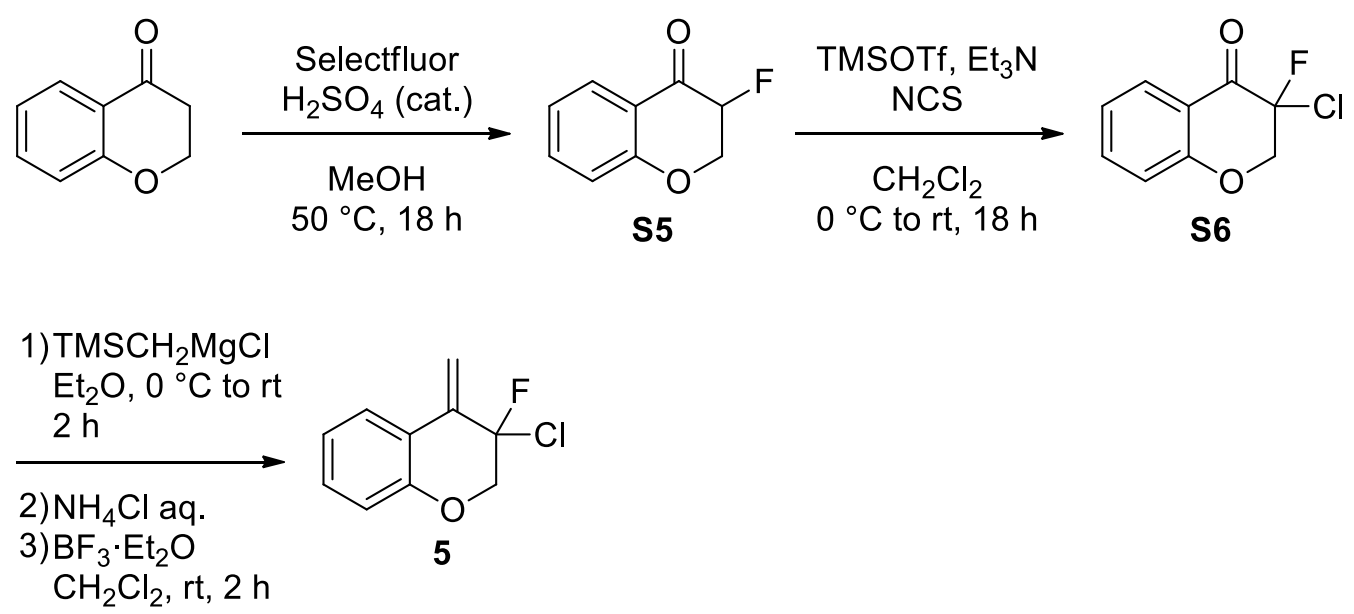

3-Fluorochroman-4-one (S5). To a solution of 4-chromanone (1.12 g, $7.57 \mathrm{mmol})$ in $\mathrm{MeOH}(15 \mathrm{~mL})$ were successively added SelectFluor $(3.22 \mathrm{~g}, 9.08 \mathrm{mmol})$ and $\mathrm{H}_{2} \mathrm{SO}_{4}(40$ $\mu \mathrm{L}, 0.757 \mathrm{mmol}$ ). The resulting mixture was stirred under argon at $50{ }^{\circ} \mathrm{C}$ for 16 hours. $\mathrm{Et}_{2} \mathrm{O}$ was added and the mixture was successively washed with water (1x) and brine (1x), then dried over $\mathrm{MgSO}_{4}$ and concentrated in vacuo. The desired product (1.22 g, 97\%) was isolated as a white solid by flash chromatography using 10\% EtOAc/hexanes. Spectroscopic data was in agreement with the literature. ${ }^{5}$

3-Chloro-3-fluorochroman-4-one (S6). The $\alpha$-chloro- $\alpha$-fluoroketone $\mathbf{S 6}$ was prepared following the general procedure for the electrophilic chlorination of S5. On a $7.07 \mathrm{mmol}$ scale, the desired product $(1.25 \mathrm{~g}, 88 \%)$ was isolated as a white solid by flash chromatography using 5\% EtOAc/hexanes. mp 52-54 ${ }^{\circ} \mathrm{C}$; IR (ATR, ZnSe) $v=3073$, 2920, 1705, 1606, 1467, 1259, 1148, 1055, 840, $752 \mathrm{~cm}^{-1} ;{ }^{1} \mathrm{H}$ NMR $\left(500 \mathrm{MHz}, \mathrm{CDCl}_{3}\right) \delta$ 7.99 (dd, 1H, $J=7.8,0.9 \mathrm{~Hz}$ ), 7.60 (m, 1H), 7.16 (t, 1H, $J=7.6 \mathrm{~Hz}$ ), 7.07 (d, $1 \mathrm{H} J=8.4$ $\mathrm{Hz})$, 4.66-4.65 (m, 2H); ${ }^{19} \mathrm{~F}$ NMR $\left(470 \mathrm{MHz}, \mathrm{CDCl}_{3}\right) \delta-135.0(\mathrm{t}, 1 \mathrm{~F}, J=6.0 \mathrm{~Hz}) ;{ }^{13} \mathrm{C}$ NMR $\left(126 \mathrm{MHz}, \mathrm{CDCl}_{3}\right) \delta 180.6\left(\mathrm{~d}, J_{\mathrm{C}-\mathrm{F}}=21.0 \mathrm{~Hz}\right), 160.4,137.6,128.8\left(\mathrm{~d}, J_{\mathrm{C}-\mathrm{F}}=1.0\right.$ $\mathrm{Hz}), 123.2,118.3,117.8,100.5\left(\mathrm{~d}, J_{\mathrm{C}-\mathrm{F}}=256 \mathrm{~Hz}\right), 73.0\left(\mathrm{~d}, J_{\mathrm{C}-\mathrm{F}}=31.1 \mathrm{~Hz}\right)$; HRMS-ESI calcd for $\mathrm{C}_{9} \mathrm{H}_{7} \mathrm{ClFO}_{2}[\mathrm{M}+\mathrm{H}]^{+}$201.0113; found 201.0106. 
3-Chloro-3-fluoro-4-methylenechroman (5). The 3-chloro-3-fluoropropene 5 was prepared following the general procedure for the Peterson olefination of S6. On a 6.04 mmol scale, the desired product (699 $\mathrm{mg}, 58 \%$ ) was isolated as a colorless oil by flash chromatography using pentane. IR (ATR, ZnSe) $v=3068,2915,1608,1482,1266,1225$, 1063, 912, 834, $749 \mathrm{~cm}^{-1} ;{ }^{1} \mathrm{H}$ NMR $\left(500 \mathrm{MHz}, \mathrm{CDCl}_{3}\right) \delta 7.57(\mathrm{~d}, 1 \mathrm{H}, J=7.9 \mathrm{~Hz}), 7.26(\mathrm{t}$, $1 \mathrm{H}, J=7.4 \mathrm{~Hz}), 7.02(\mathrm{t}, 1 \mathrm{H}, J=7.5 \mathrm{~Hz}), 6.95(\mathrm{~d}, 1 \mathrm{H}, J=8.2 \mathrm{~Hz}), 5.81(\mathrm{~s}, 1 \mathrm{H}), 5.77(\mathrm{~s}$, 1H), 4.39-4.31 (m, 2H); ${ }^{19} \mathrm{~F}$ NMR (470 MHz, $\left.\mathrm{CDCl}_{3}\right) \delta-116.6(\mathrm{~m}, 1 \mathrm{~F}) ;{ }^{13} \mathrm{C} \mathrm{NMR}(126$ $\left.\mathrm{MHz} \mathrm{CDCl}_{3}\right) \delta 152.4,138.5\left(\mathrm{~d}, J_{\mathrm{C}-\mathrm{F}}=18.5 \mathrm{~Hz}\right), 130.6,124.9\left(\mathrm{~d}, J_{\mathrm{C}-\mathrm{F}}=1.3 \mathrm{~Hz}\right), 122.4$, $118.4\left(\mathrm{~d}, J_{\mathrm{C}-\mathrm{F}}=2.3 \mathrm{~Hz}\right), 117.8,108.9\left(\mathrm{~d}, J_{\mathrm{C}-\mathrm{F}}=8.0 \mathrm{~Hz}\right), 104.5\left(\mathrm{~d}, J_{\mathrm{C}-\mathrm{F}}=248 \mathrm{~Hz}\right), 72.1(\mathrm{~d}$, $J_{\mathrm{C}-\mathrm{F}}=31.0 \mathrm{~Hz}$ ); HRMS-ESI calcd for $\mathrm{C}_{11} \mathrm{H}_{11} \mathrm{ClF}[\mathrm{M}+\mathrm{H}]^{+}$199.0320; found 199.0303.<smiles>CC1(C)C=CC(=O)CC1</smiles>

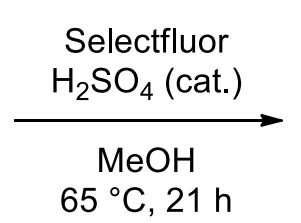

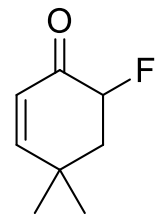

S7

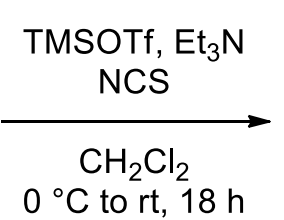

$0{ }^{\circ} \mathrm{C}$ to $\mathrm{rt}, 18 \mathrm{~h}$

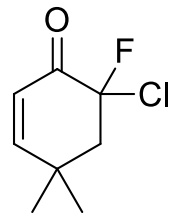

S8
1) $\mathrm{TMSCH}_{2} \mathrm{MgCl}$

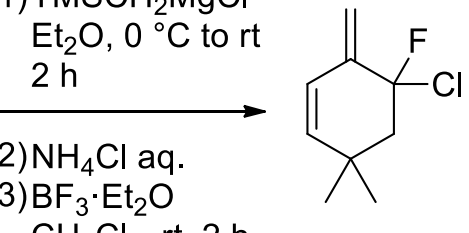

$\mathrm{CH}_{2} \mathrm{Cl}_{2}, \mathrm{rt}, 2 \mathrm{~h} \quad 6$

6-Fluoro-4,4-dimethylcyclohex-2-enone (S7). To a solution of 4,4-dimethyl-2cyclohexen-1-one $(1.0 \mathrm{~mL}, 7.60 \mathrm{mmol})$ in $\mathrm{MeOH}(4 \mathrm{~mL})$ were successively added SelectFluor $(3.63 \mathrm{~g}, 10.2 \mathrm{mmol})$ and $\mathrm{H}_{2} \mathrm{SO}_{4}(40 \mu \mathrm{L}, 0.760 \mathrm{mmol})$. The resulting mixture was stirred under argon at $65{ }^{\circ} \mathrm{C}$ for 21 hours. The mixture was cooled to ambient temperature, filtered to remove the solids, and then concentrated in vacuo. $\mathrm{Et}_{2} \mathrm{O}$ was added and the resulting solution was successively washed with water $(1 \mathrm{x})$ and brine $(1 \mathrm{x})$, then dried over $\mathrm{MgSO}_{4}$ and concentrated in vacuo. The $\alpha$-fluoroketone $\mathbf{S 7}$ (312 mg, 26\%) was isolated as a colorless solid by flash chromatography using 10\% EtOAc/hexanes. Spectroscopic data was in agreement with the literature. ${ }^{6}$

6-Chloro-6-fluoro-4,4-dimethylcyclohex-2-enone (S8). The $\alpha$-chloro- $\alpha$-fluoroketone S8 was prepared following the general procedure for the electrophilic chlorination of S7. On a $2.31 \mathrm{mmol}$ scale, the desired product $(301 \mathrm{mg}, 74 \%$ ) was isolated as a colorless oil by flash chromatography using $10 \%$ EtOAc/hexanes. mp 56-58 ${ }^{\circ} \mathrm{C}$; IR (ATR, ZnSe) $v=$ 2965, 2875, 1700, 1613, 1118, 1071, 964, 817, 778, $724 \mathrm{~cm}^{-1} ;{ }^{1} \mathrm{H}$ NMR (500 MHz, $\left.\mathrm{CDCl}_{3}\right) \delta 6.73(\mathrm{~d}, 1 \mathrm{H}, J=10.3 \mathrm{~Hz}), 6.01(\mathrm{dd}, 1 \mathrm{H}, J=10.3,3.0 \mathrm{~Hz}), 2.61(\mathrm{~d}, J=13.3 \mathrm{~Hz})$, 
$1.36(\mathrm{~s}, 3 \mathrm{H}), 1.27(\mathrm{~s}, 3 \mathrm{H}) ;{ }^{19} \mathrm{~F} \mathrm{NMR}\left(470 \mathrm{MHz}, \mathrm{CDCl}_{3}\right) \delta-109.5(\mathrm{t}, 1 \mathrm{~F}, J=13.2 \mathrm{~Hz}) ;{ }^{13} \mathrm{C}$ NMR $\left(126 \mathrm{MHz}, \mathrm{CDCl}_{3}\right) \delta 185.4\left(\mathrm{~d}, J_{\mathrm{C}-\mathrm{F}}=21.7 \mathrm{~Hz}\right), 160.2\left(\mathrm{~d}, J_{\mathrm{C}-\mathrm{F}}=0.7 \mathrm{~Hz}\right), 122.9(\mathrm{~d}$, $\left.J_{\mathrm{C}-\mathrm{F}}=0.6 \mathrm{~Hz}\right), 104.0\left(\mathrm{~d}, J_{\mathrm{C}-\mathrm{F}}=252 \mathrm{~Hz}\right), 49.4\left(\mathrm{~d}, J_{\mathrm{C}-\mathrm{F}}=19.7 \mathrm{~Hz}\right), 35.4\left(\mathrm{~d}, J_{\mathrm{C}-\mathrm{F}}=5.7 \mathrm{~Hz}\right)$, $30.7\left(\mathrm{~d}, J_{\mathrm{C}-\mathrm{F}}=1.7 \mathrm{~Hz}\right), 27.7\left(\mathrm{~d}, J_{\mathrm{C}-\mathrm{F}}=0.6 \mathrm{~Hz}\right)$; HRMS-ESI calcd for $\mathrm{C}_{8} \mathrm{H}_{11} \mathrm{ClFO}[\mathrm{M}+\mathrm{H}]^{+}$ 177.0477; found 177.0475.

5-Chloro-5-fluoro-3,3-dimethyl-6-methylenecyclohex-1-ene (6). The 3-chloro-3fluoropropene 5 was prepared following the general procedure for the Peterson olefination of S8. On a $1.24 \mathrm{mmol}$ scale, the desired product ( $48 \mathrm{mg}, 22 \%$ ) was isolated as a colorless oil by flash chromatography using pentane. IR (ATR, ZnSe) $v=3025$, 2960, 2870, 1472, 1364, 1138, 1065, 901, 791, $692 \mathrm{~cm}^{-1} ;{ }^{1} \mathrm{H}$ NMR $\left(500 \mathrm{MHz}, \mathrm{CDCl}_{3}\right) \delta$ 6.01 (dd, 1H, $J=9.9,2.5 \mathrm{~Hz}$ ), 5.69 (s, 1H), 5.62 (d, 1H, $J=9.9 \mathrm{~Hz}), 5.19$ (s, 1H), 2.39$2.28(\mathrm{~m}, 2 \mathrm{H}), 1.21(\mathrm{~s}, 3 \mathrm{H}), 1.13(\mathrm{~s}, 3 \mathrm{H}) ;{ }^{19} \mathrm{~F}$ NMR $\left(470 \mathrm{MHz}, \mathrm{CDCl}_{3}\right) \delta-91.1(\mathrm{dd}, 1 \mathrm{~F}, J=$ 19.0, $11.4 \mathrm{~Hz}) ;{ }^{13} \mathrm{C} \mathrm{NMR}\left(126 \mathrm{MHz}, \mathrm{CDCl}_{3}\right) \delta 141.9\left(\mathrm{~d}, J_{\mathrm{C}-\mathrm{F}}=19.0 \mathrm{~Hz}\right), 139.3\left(\mathrm{~d}, J_{\mathrm{C}-\mathrm{F}}=\right.$ $2.0 \mathrm{~Hz}), 122.9\left(\mathrm{~d}, J_{\mathrm{C}-\mathrm{F}}=2.2 \mathrm{~Hz}\right), 114.7\left(\mathrm{~d}, J_{\mathrm{C}-\mathrm{F}}=6.7 \mathrm{~Hz}\right), 109.8\left(\mathrm{~d}, J_{\mathrm{C}-\mathrm{F}}=244 \mathrm{~Hz}\right), 50.6$ $\left(\mathrm{d}, J_{\mathrm{C}-\mathrm{F}}=20.2 \mathrm{~Hz}\right), 35.0\left(\mathrm{~d}, J_{\mathrm{C}-\mathrm{F}}=4.4 \mathrm{~Hz}\right), 30.4\left(\mathrm{~d}, J_{\mathrm{C}-\mathrm{F}}=2.7 \mathrm{~Hz}\right), 29.1$; HRMS-ESI calcd for $\mathrm{C}_{9} \mathrm{H}_{13} \mathrm{ClF}[\mathrm{M}+\mathrm{H}]^{+}$175.0684; found 175.0655.
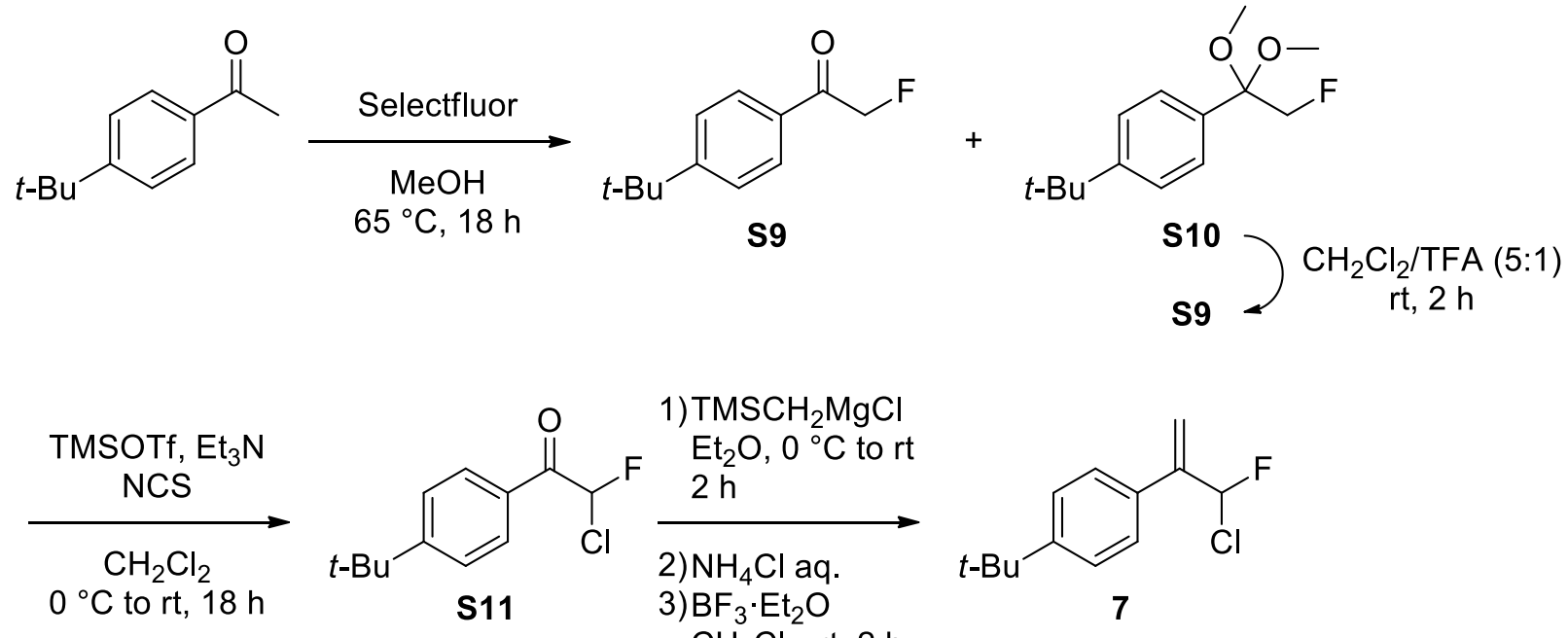

1-(4-(tert-Butyl)phenyl)-2-fluoroethanone (S9). To a solution of 4'-tertbutylacetophenone $(2.34 \mathrm{~g}, 13.1 \mathrm{mmol})$ in $\mathrm{MeOH}(130 \mathrm{~mL})$ was added SelectFluor (4.88 $\mathrm{g}, 13.8 \mathrm{mmol})$. The resulting mixture was stirred under argon at $65^{\circ} \mathrm{C}$ for 18 hours. $\mathrm{Et}_{2} \mathrm{O}$ 
was added and the mixture was successively washed with water $(1 \mathrm{x})$ and brine $(1 \mathrm{x})$, then dried over $\mathrm{MgSO}_{4}$ and concentrated. The $\alpha$-fluoroketone $\mathbf{S 9}$ and the ( $\alpha$ fluoro)dimethylacetal S10 side-product were then separated and isolated by flash chromatography using 3\% EtOAc/hexanes. S11 was subsequently taken up in a 5:1 $\mathrm{CH}_{2} \mathrm{Cl}_{2} / \mathrm{TFA}$ mixture and stirred at ambient temperature for two hours. The solution was washed with water $(3 \mathrm{x})$, dried over $\mathrm{MgSO}_{4}$ and concentrated. The resulting $\alpha$ fluoroketone S9 was isolated by flash chromatography using 3\% EtOAc/hexanes. Overall, the desired product $(1.94 \mathrm{~g}, 76 \%)$ was isolated as a colorless solid. Spectroscopic data was in agreement with the literature. ${ }^{7}$

1-(tert-Butyl)-4-(2-fluoro-1,1-dimethoxyethyl)benzene (S10). Colorless solid; mp 41$44{ }^{\circ} \mathrm{C}$; IR (ATR, ZnSe) $v=2953,2838,1460,1293,1113,1090,1063,1033,972,674$ $\mathrm{cm}^{-1} ;{ }^{1} \mathrm{H}$ NMR $\left(500 \mathrm{MHz}, \mathrm{CDCl}_{3}\right) \delta 7,43(\mathrm{~d}, 2 \mathrm{H}, J=8.2 \mathrm{~Hz}), 7.39(\mathrm{~d}, 2 \mathrm{H}, J=8.3 \mathrm{~Hz})$, $4.49(\mathrm{~d}, 2 \mathrm{H}, J=47.2 \mathrm{~Hz}), 3.29(\mathrm{~s}, 6 \mathrm{H}), 1.32(\mathrm{~s}, 9 \mathrm{H}) ;{ }^{19} \mathrm{~F} \mathrm{NMR}\left(376 \mathrm{MHz}, \mathrm{CDCl}_{3}\right)$ $\delta-228.1(\mathrm{t}, 1 \mathrm{~F}, J=47.1 \mathrm{~Hz}) ;{ }^{13} \mathrm{C}$ NMR $\left(126 \mathrm{MHz}, \mathrm{CDCl}_{3}\right) \delta 151.5,135.1,127.0,125.3$, $100.6\left(\mathrm{~d}, J_{\mathrm{C}-\mathrm{F}}=19.8 \mathrm{~Hz}\right), 83.8\left(\mathrm{~d}, J_{\mathrm{C}-\mathrm{F}}=178 \mathrm{~Hz}\right), 49.3\left(\mathrm{~d}, J_{\mathrm{C}-\mathrm{F}}=1.6 \mathrm{~Hz}\right), 34.7,31.5$; HRMS-ESI calcd for $\mathrm{C}_{13} \mathrm{H}_{19} \mathrm{FO}[\mathrm{M}-\mathrm{MeO}]^{+}$209.1342; found 209.1350.

1-(4-(tert-Butyl)phenyl)-2-chloro-2-fluoroethanone (S11). The $\alpha$-chloro- $\alpha$-fluoroketone S11 was prepared following the general procedure for the electrophilic chlorination of S9. On a $5.10 \mathrm{mmol}$ scale, the desired product $(1.06 \mathrm{~g}, 91 \%)$ was isolated as a colorless oil by flash chromatography using 3\% EtOAc/hexanes. IR (ATR, ZnSe) $v=2965,2870$, 1706, 1605, 1412, 1283, 1101, 973, 856, $735 \mathrm{~cm}^{-1}$; ${ }^{1} \mathrm{H}$ NMR $\left(500 \mathrm{MHz}, \mathrm{CDCl}_{3}\right) \delta 8.01$ $(\mathrm{d}, 2 \mathrm{H}, J=8.1 \mathrm{~Hz}), 7.54(\mathrm{~d}, 2 \mathrm{H}, J=8.1 \mathrm{~Hz}), 6.82(\mathrm{~d}, 1 \mathrm{H}, J=50.7 \mathrm{~Hz}), 1.36(\mathrm{~s}, 9 \mathrm{H}) ;{ }^{19} \mathrm{~F}$ NMR (470 MHz, $\left.\mathrm{CDCl}_{3}\right) \delta-146.3(\mathrm{~d}, 1 \mathrm{~F}, J=50.8 \mathrm{~Hz}) ;{ }^{13} \mathrm{C} \mathrm{NMR}\left(126 \mathrm{MHz}, \mathrm{CDCl}_{3}\right) \delta$ $187.1\left(\mathrm{~d}, J_{\mathrm{C}-\mathrm{F}}=22.5 \mathrm{~Hz}\right), 159.0,129.8\left(\mathrm{~d}, J_{\mathrm{C}-\mathrm{F}}=2.6 \mathrm{~Hz}\right), 128.7\left(\mathrm{~d}, J_{\mathrm{C}-\mathrm{F}}=1.4 \mathrm{~Hz}\right), 126.1$, $95.4\left(\mathrm{~d}, J_{\mathrm{C}-\mathrm{F}}=257 \mathrm{~Hz}\right), 35.5,31.1$; HRMS-ESI calcd for $\mathrm{C}_{12} \mathrm{H}_{15} \mathrm{ClFO}[\mathrm{M}+\mathrm{H}]^{+} 229.0790$; found 229.0798 .

1-(tert-Butyl)-4-(3-chloro-3-fluoroprop-1-en-2-yl)benzene (7). The 3-chloro-3fluoropropene 7 was prepared following the general procedure for the Peterson olefination of S11. On a $6.63 \mathrm{mmol}$ scale, the desired product $(1.24 \mathrm{~g}, 83 \%)$ was isolated as a colorless oil by flash chromatography using hexanes. IR (ATR, ZnSe) $v=3090$, $2963,2869,1514,1364,1270,1049,939,838,783 \mathrm{~cm}^{-1} ;{ }^{1} \mathrm{H}$ NMR $\left(500 \mathrm{MHz}, \mathrm{CDCl}_{3}\right) \delta$ 
$7.41(\mathrm{~m}, 4 \mathrm{H}), 6.88(\mathrm{~d}, 1 \mathrm{H}, J=49.7 \mathrm{~Hz}), 5.64(\mathrm{~s}, 1 \mathrm{H}), 5.61(\mathrm{~d}, 1 \mathrm{H}, J=2.0 \mathrm{~Hz}), 1.33(\mathrm{~s}$, $9 \mathrm{H}) ;{ }^{19} \mathrm{~F}$ NMR $\left(470 \mathrm{MHz}, \mathrm{CDCl}_{3}\right) \delta-130.6(\mathrm{~d}, 1 \mathrm{~F}, J=49.7 \mathrm{~Hz}) ;{ }^{13} \mathrm{C}$ NMR $(126 \mathrm{MHz}$, $\left.\mathrm{CDCl}_{3}\right) \delta 151.9,144.7\left(\mathrm{~d}, J_{\mathrm{C}-\mathrm{F}}=17.7 \mathrm{~Hz}\right), 132.5\left(\mathrm{~d}, J_{\mathrm{C}-\mathrm{F}}=1.6 \mathrm{~Hz}\right), 126.8,125.6,116.6$ $\left(\mathrm{d}, J_{\mathrm{C}-\mathrm{F}}=9.5 \mathrm{~Hz}\right), 100.7\left(\mathrm{~d}, J_{\mathrm{C}-\mathrm{F}}=245 \mathrm{~Hz}\right), 34.8,31.4$; HRMS-ESI calcd for $\mathrm{C}_{13} \mathrm{H}_{17} \mathrm{ClF}[\mathrm{M}+\mathrm{H}]^{+}$227.0997; found 227.0989.

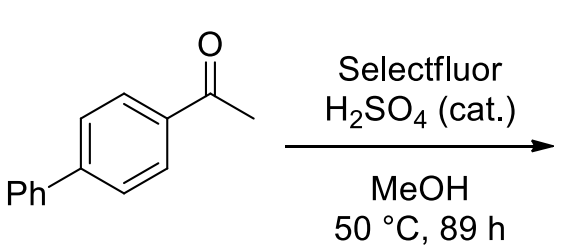

$50{ }^{\circ} \mathrm{C}, 89 \mathrm{~h}$

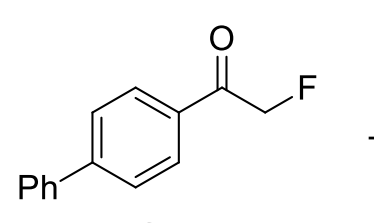

S12<smiles>COC(CF)(OC)c1ccc(-c2ccccc2)cc1</smiles>

$\mathrm{S} 13$
$\mathbf{s 1 2}$$\underset{\mathrm{rt}, 2 \mathrm{~h}}{\mathrm{CH}_{2} \mathrm{Cl}_{2} / \mathrm{TFA}(5: 1)}$

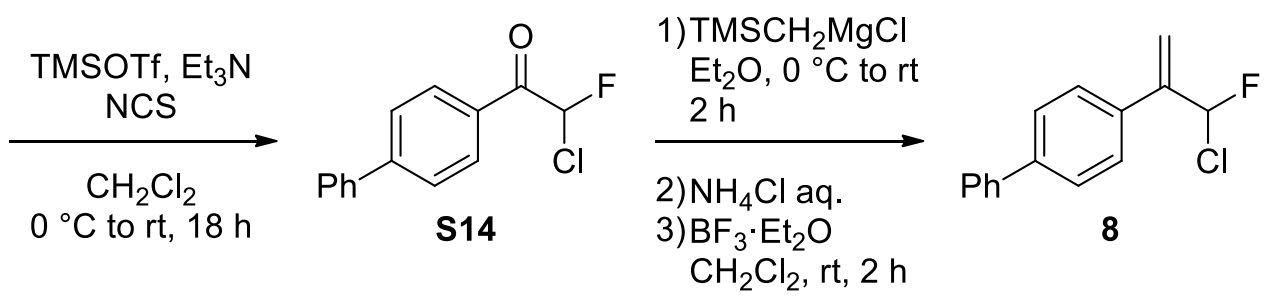

1-([1,1'-Biphenyl]-4-yl)-2-fluoroethanone (S12). To a solution of 4-acetylbiphenyl (1.49 g, $7.57 \mathrm{mmol})$ in $\mathrm{MeOH}(50 \mathrm{~mL})$ were successively added SelectFluor $(3.22 \mathrm{~g}, 9.08$ mmoL) and $\mathrm{H}_{2} \mathrm{SO}_{4}(40 \mu \mathrm{L}, 0.757 \mathrm{mmoL})$. The resulting mixture was stirred under argon at $50{ }^{\circ} \mathrm{C}$ for 89 hours. $\mathrm{Et}_{2} \mathrm{O}$ was added and the mixture was successively washed with water $(1 \mathrm{x})$ and brine $(1 \mathrm{x})$, then dried over $\mathrm{MgSO}_{4}$ and concentrated. The $\alpha$-fluoroketone S12 and the ( $\alpha$-fluoro)dimethylacetal S13 side-product were then separated and isolated by flash chromatography using 5\% EtOAc/hexanes. S13 was subsequently taken up in a 5:1 $\mathrm{CH}_{2} \mathrm{Cl}_{2} / \mathrm{TFA}$ mixture and stirred at ambient temperature for two hours. The solution was washed with water $(3 \mathrm{x})$, dried over $\mathrm{MgSO}_{4}$ and concentrated. The resulting $\alpha$ fluoroketone S12 was isolated by flash chromatography using 5\% EtOAc/hexanes. Overall, the desired product (432 $\mathrm{mg}, 27 \%$ ) was isolated as an off-white solid. Spectroscopic data was in agreement with the literature. ${ }^{8}$ 
4-(2-Fluoro-1,1-dimethoxyethyl)-1,1'-biphenyl (S13). White solid; mp 79-81 ${ }^{\circ} \mathrm{C}$; IR $(\mathrm{ATR}, \mathrm{ZnSe}) v=3064,2940,2836,1485,1294,1146,1058,971,739,695 \mathrm{~cm}^{-1} ;{ }^{1} \mathrm{H}$ NMR (500 MHz, $\left.\mathrm{CDCl}_{3}\right) \delta 7.63-7.58(\mathrm{~m}, 6 \mathrm{H}), 7.44(\mathrm{t}, 2 \mathrm{H}, J=7.5 \mathrm{~Hz}), 7.35(\mathrm{t}, 2 \mathrm{H}, J=$ $7.3 \mathrm{~Hz}), 4.54(\mathrm{~d}, 1 \mathrm{H}, J=47.2 \mathrm{~Hz}), 3.32(\mathrm{~s}, 6 \mathrm{H}) ;{ }^{19} \mathrm{~F} \mathrm{NMR}\left(470 \mathrm{MHz}, \mathrm{CDCl}_{3}\right) \delta-228.6(\mathrm{t}$, $1 \mathrm{~F}, J=47.2 \mathrm{~Hz}) ;{ }^{13} \mathrm{C} \mathrm{NMR}\left(126 \mathrm{MHz}, \mathrm{CDCl}_{3}\right) \delta 141.5,140.8,137.2,128.9,127.8$, 127.6, 127.3, 127.1, $100.6\left(\mathrm{~d}, J_{\mathrm{C}-\mathrm{F}}=20.0 \mathrm{~Hz}\right), 83.5\left(\mathrm{~d}, J_{\mathrm{C}-\mathrm{F}}=178 \mathrm{~Hz}\right), 49.4$; HRMS-ESI calcd for $\mathrm{C}_{15} \mathrm{H}_{14} \mathrm{FO}[\mathrm{M}-\mathrm{MeO}]^{+} 229.1023$; found 229.0998 .

1-([1,1'-Biphenyl]-4-yl)-2-chloro-2-fluoroethanone (S14). The $\alpha$-chloro- $\alpha$-fluoroketone S14 was prepared following the general procedure for the electrophilic chlorination of S12. On a $1.99 \mathrm{mmol}$ scale, the desired product (396 $\mathrm{mg}, 80 \%$ ) was isolated as an offwhite solid by flash chromatography using $5 \%$ EtOAc/hexanes. mp $84-87{ }^{\circ} \mathrm{C}$; IR (ATR, $\mathrm{ZnSe}) v=3066,2957,1692,1600,1408,1197,1090,965,859,689 \mathrm{~cm}^{-1} ;{ }^{1} \mathrm{H}$ NMR (500 $\left.\mathrm{MHz}, \mathrm{CDCl}_{3}\right) \delta 8.15(\mathrm{~d}, 2 \mathrm{H}, J=8.2 \mathrm{~Hz}), 7.74(\mathrm{~d}, 2 \mathrm{H}, J=8.3 \mathrm{~Hz}), 7.64(\mathrm{~d}, 2 \mathrm{H}, J=7.5$ $\mathrm{Hz}), 7.49(\mathrm{t}, 2 \mathrm{H}, J=7.5 \mathrm{~Hz}), 7.43(\mathrm{t}, 1 \mathrm{H}, J=7.3 \mathrm{~Hz}), 6.85(\mathrm{~d}, 1 \mathrm{H}, J=50.8 \mathrm{~Hz}) ;{ }^{19} \mathrm{~F}$ NMR $\left(470 \mathrm{MHz}, \mathrm{CDCl}_{3}\right) \delta-146.1(\mathrm{~d}, 1 \mathrm{~F}, J=50.8 \mathrm{~Hz}) ;{ }^{13} \mathrm{C} \mathrm{NMR}\left(126 \mathrm{MHz}, \mathrm{CDCl}_{3}\right) \delta$ $187.1\left(\mathrm{~d}, J_{\mathrm{C}-\mathrm{F}}=22.9 \mathrm{~Hz}\right), 147.6,139.6,130.45,130.43,129.2,128.8,127.6,127.5,95.5$ $\left(\mathrm{d}, J_{\mathrm{C}-\mathrm{F}}=257 \mathrm{~Hz}\right)$; HRMS-ESI calcd for $\mathrm{C}_{14} \mathrm{H}_{10} \mathrm{ClFNaO}[\mathrm{M}+\mathrm{Na}]^{+} 271.0296$; found 271.0275 .

4-(3-Chloro-3-fluoroprop-1-en-2-yl)-1,1'-biphenyl (8). The 3-chloro-3-fluoropropene 8 was prepared following the general procedure for the Peterson olefination of S14. On a $1.50 \mathrm{mmol}$ scale, the desired product $(301 \mathrm{mg}, 82 \%$ ) was isolated as a white solid by flash chromatography using pentane. mp 50-51 ${ }^{\circ} \mathrm{C}$; IR (ATR, ZnSe) $v=3034,2979$, $1487,1406,1261,1108,1048,924,843,771 \mathrm{~cm}^{-1} ;{ }^{1} \mathrm{H}$ NMR $\left(500 \mathrm{MHz}, \mathrm{CDCl}_{3}\right) \delta 7.63-$ $7.60(\mathrm{~m}, 4 \mathrm{H}), 7.56(\mathrm{~d}, 2 \mathrm{H}, J=8.0 \mathrm{~Hz}), 7.45(\mathrm{t}, 2 \mathrm{H}, J=7.5 \mathrm{~Hz}), 7.37(\mathrm{t}, 1 \mathrm{H}, J=7.2 \mathrm{~Hz})$, $6.92(\mathrm{~d}, 1 \mathrm{H}, J=49.6 \mathrm{~Hz}), 5.70(\mathrm{~s}, 1 \mathrm{H}), 5.68(\mathrm{~d}, 1 \mathrm{H}, J=2.4 \mathrm{~Hz}) ;{ }^{19} \mathrm{~F}$ NMR $(470 \mathrm{MHz}$, $\left.\mathrm{CDCl}_{3}\right) \delta-130.4(\mathrm{~d}, 1 \mathrm{~F}, J=49.6 \mathrm{~Hz}) ;{ }^{13} \mathrm{C} \mathrm{NMR}\left(126 \mathrm{MHz}, \mathrm{CDCl}_{3}\right) \delta 144.6\left(\mathrm{~d}, J_{\mathrm{C}-\mathrm{F}}=\right.$ $17.9 \mathrm{~Hz}), 141.7,140.5,134.4\left(\mathrm{~d}, J_{\mathrm{C}-\mathrm{F}}=1.4 \mathrm{~Hz}\right), 129.0,127.7,127.6\left(\mathrm{~d}, J_{\mathrm{C}-\mathrm{F}}=0.7 \mathrm{~Hz}\right)$, 127.4, 127.2, $117.3\left(\mathrm{~d}, J_{\mathrm{C}-\mathrm{F}}=9.3 \mathrm{~Hz}\right), 100.7\left(\mathrm{~d}, J_{\mathrm{C}-\mathrm{F}}=232 \mathrm{~Hz}\right)$; HRMS-ESI calcd for $\mathrm{C}_{15} \mathrm{H}_{13} \mathrm{ClF}[\mathrm{M}+\mathrm{H}]^{+}$247.0684; found 247.0679. 


\section{Synthesis of monofluoroalkenes}

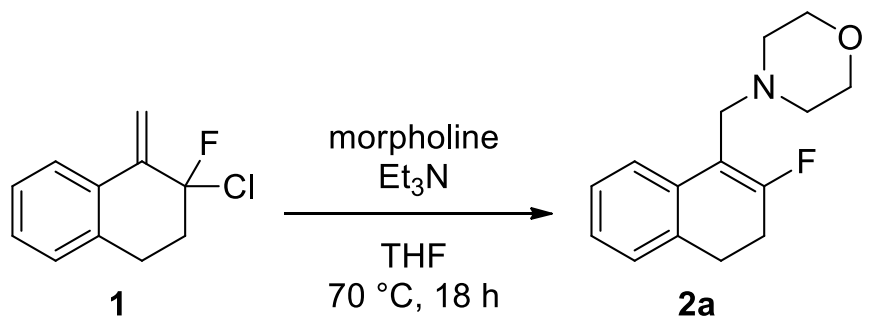

4-((2-Fluoro-3,4-dihydronaphthalen-1-yl)methyl)morpholine (2a). To an argonflushed vial was added a solution of $1(50 \mathrm{mg}, 0.254 \mathrm{mmol})$ in THF (2.5 mL), followed by morpholine $(44 \mu \mathrm{L}, 0.509 \mathrm{mmol})$ ) and $\mathrm{Et}_{3} \mathrm{~N}(35 \mu \mathrm{L}, 0.254 \mathrm{mmol})$. The resulting mixture was stirred at $70{ }^{\circ} \mathrm{C}$ for 18 hours. Water was added and the mixture was extracted with $\mathrm{Et}_{2} \mathrm{O}(3 \mathrm{x})$. The organic layers were combined, washed with brine $(1 \mathrm{x})$, dried over $\mathrm{Na}_{2} \mathrm{SO}_{4}$ and concentrated in vacuo. The desired product (60 mg, 96\%) was isolated as a colorless oil by flash chromatography using $15 \% \mathrm{Et}_{2} \mathrm{O} / \mathrm{hexanes}$. Spectroscopic data was in agreement with the literature. ${ }^{9}{ }^{1} \mathrm{H}$ NMR $\left(500 \mathrm{MHz}, \mathrm{CDCl}_{3}\right) \delta$ $7.56(\mathrm{~d}, 1 \mathrm{H}, J=7.7 \mathrm{~Hz}), 7.20(\mathrm{~m}, 1 \mathrm{H}), 7.13-7.10(\mathrm{~m}, 2 \mathrm{H}), 3.68(\mathrm{t}, 4 \mathrm{H}, J=4.4 \mathrm{~Hz}), 3.41$ (s, 2H), $2.97(\mathrm{td}, 2 \mathrm{H}, J=8.2,2.4 \mathrm{~Hz}), 2.57(\mathrm{~m}, 2 \mathrm{H}), 2.50(\mathrm{~m}, 4 \mathrm{H})$.

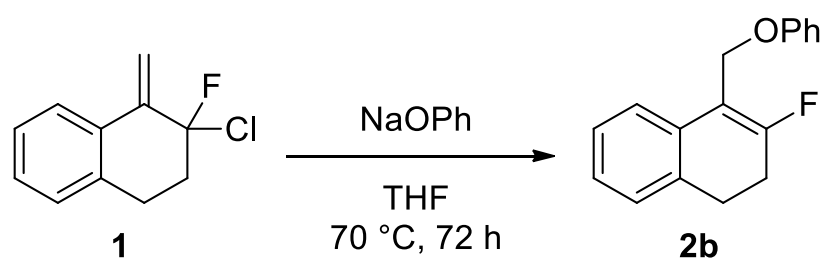

3-Fluoro-4-(phenoxymethyl)-1,2-dihydronaphthalene (2b). An argon-flushed vial was charged with $\mathrm{NaH}(60 \%$ in mineral oil, $20 \mathrm{mg}, 0.509 \mathrm{mmol})$ and THF $(0.5 \mathrm{~mL})$. A solution of phenol (48 mg, $0.509 \mathrm{mmol})$ and THF (1 mL) was added slowly, and the resulting mixture was stirred at ambient temperature for 15 minutes. A solution of 1 (50 $\mathrm{mg}, 0.254 \mathrm{mmol})$ in THF ( $1 \mathrm{ml})$ was added. The resulting mixture was stirred at $70{ }^{\circ} \mathrm{C}$ for 72 hours. Water was added and the mixture was extracted with $\mathrm{Et}_{2} \mathrm{O}(3 \mathrm{x})$. The organic layers were combined, washed with brine $(1 \mathrm{x})$, dried over $\mathrm{Na}_{2} \mathrm{SO}_{4}$ and concentrated in 
vасио. The desired product $(52 \mathrm{mg}, 80 \%)$ was isolated as a colorless oil by flash chromatography using hexanes. IR (ATR, ZnSe) $v=3061,2944,1683,1597,1493,1225$, 1007, 833, 750, $689 \mathrm{~cm}^{-1} ;{ }^{1} \mathrm{H}$ NMR (500 MHz, $\mathrm{CDCl}_{3}$ ) $\delta$ 7.35-7.29 (m, 3H), 7.20-7.12 (m, 3H), 7.02-6.96 (m, 3H), 4.99 (d, 2H, $J=1.9 \mathrm{~Hz}), 3.01(\mathrm{td}, 2 \mathrm{H}, J=8.2,2.3 \mathrm{~Hz}), 2.63$ (m, 2H); ${ }^{19} \mathrm{~F}$ NMR $\left(470 \mathrm{MHz}, \mathrm{CDCl}_{3}\right) \delta-100,0(\mathrm{~s}, 1 \mathrm{~F}) ;{ }^{13} \mathrm{C} \mathrm{NMR}\left(126 \mathrm{MHz}, \mathrm{CDCl}_{3}\right) \delta$ $161.8\left(\mathrm{~d}, J_{\mathrm{C}-\mathrm{F}}=273 \mathrm{~Hz}\right), 158.7,133.1\left(\mathrm{~d}, J_{\mathrm{C}-\mathrm{F}}=5.4 \mathrm{~Hz}\right), 132.7,129.6,127.5,127.0$, $126.6\left(\mathrm{~d}, J_{\mathrm{C}-\mathrm{F}}=2.2 \mathrm{~Hz}\right), 124.0\left(\mathrm{~d}, J_{\mathrm{C}-\mathrm{F}}=6.6 \mathrm{~Hz}\right), 121.1,115.0,110.9\left(\mathrm{~d}, J_{\mathrm{C}-\mathrm{F}}=10.9 \mathrm{~Hz}\right)$, $60.0\left(\mathrm{~d}, J_{\mathrm{C}-\mathrm{F}}=7.5 \mathrm{~Hz}\right), 28.5\left(\mathrm{~d}, J_{\mathrm{C}-\mathrm{F}}=7.3 \mathrm{~Hz}\right), 25.1\left(\mathrm{~d}, J_{\mathrm{C}-\mathrm{F}}=24.0 \mathrm{~Hz}\right)$; HRMS-ESI calcd for $\mathrm{C}_{17} \mathrm{H}_{16} \mathrm{FO}[\mathrm{M}+\mathrm{H}]^{+}$255.1180; found 255.1183.

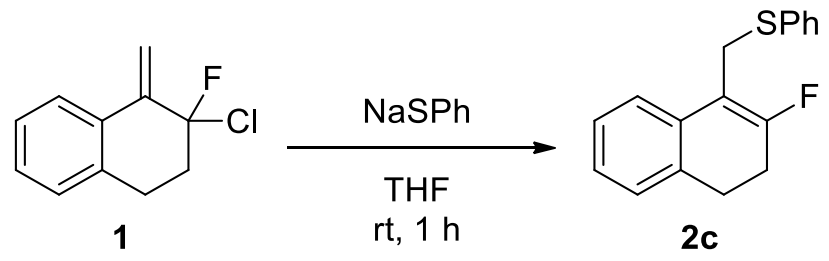

((2-Fluoro-3,4-dihydronaphthalen-1-yl)methyl)(phenyl)sulfane (2c). An argonflushed vial was charged with $\mathrm{NaH}(60 \%$ in mineral oil, $20 \mathrm{mg}, 0.509 \mathrm{mmol})$ and THF $(1.5 \mathrm{~mL})$. Thiophenol $(52 \mu \mathrm{L}, 0.509 \mathrm{mmol})$ was added slowly, and the resulting mixture was stirred at ambient temperature for 5 minutes. A solution of $1(50 \mathrm{mg}, 0.254 \mathrm{mmol})$ in THF ( $1 \mathrm{ml})$ was added. The resulting mixture was stirred at ambient temperature for 1 hour. $\mathrm{NaOH}(0.1 \mathrm{M})$ was added and the mixture was extracted with $\mathrm{Et}_{2} \mathrm{O}(2 \mathrm{x})$. The organic layers were combined, washed with brine (1x), dried over $\mathrm{MgSO}_{4}$ and concentrated in vacuo. The desired product (67 mg, 97\%) was isolated as a colorless oil by flash chromatography using a solvent gradient ranging from $100 \%$ hexanes to $5 \%$ EtOAc/hexanes. Spectroscopic data was in agreement with the literature. ${ }^{10}{ }^{1} \mathrm{H}$ NMR (500 $\left.\mathrm{MHz}, \mathrm{CDCl}_{3}\right) \delta$ 7.40-7.35 (m, 3H), 7.28 (t, $\left.2 \mathrm{H}, J=7.5 \mathrm{~Hz}\right), 7.25-7.21(\mathrm{~m}, 2 \mathrm{H}), 7.14-7.10$ (m, 2H), 4.03 (s, 2H), $2.90(\mathrm{td}, 2 \mathrm{H}, J=8.2,2.3 \mathrm{~Hz}), 2.49(\mathrm{~m}, 2 \mathrm{H})$. 


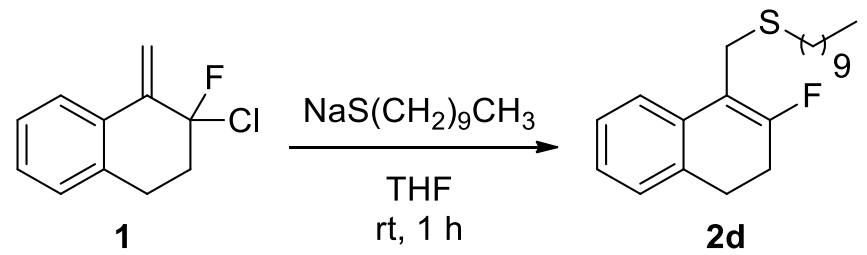

Decyl((2-fluoro-3,4-dihydronaphthalen-1-yl)methyl)sulfane (2d). An argon-flushed vial was charged with $\mathrm{NaH}(60 \%$ in mineral oil, $20 \mathrm{mg}, 0.509 \mathrm{mmol})$ and THF (1.5 mL). 1-Decanethiol $(108 \mu \mathrm{L}, 0.509 \mathrm{mmol})$ was added slowly, and the resulting mixture was stirred at ambient temperature for 5 minutes. A solution of 1 (50 mg, $0.254 \mathrm{mmol})$ in THF (1 ml) was added. The resulting mixture was stirred at ambient temperature for 1 hour. $\mathrm{NaOH}(0.1 \mathrm{M})$ was added and the mixture was extracted with $\mathrm{Et}_{2} \mathrm{O}(2 \mathrm{x})$. The organic layers were combined, washed with brine $(1 \mathrm{x})$, dried over $\mathrm{MgSO}_{4}$ and concentrated in vacuo. The desired product ( $77 \mathrm{mg}, 90 \%)$ was isolated as a colorless oil by flash chromatography using $2 \% \mathrm{Et}_{2} \mathrm{O} /$ hexanes. IR (ATR, ZnSe) $v=3061,2922,2851$, 1676, 1452, 1365, 1149, 956, 761, $726 \mathrm{~cm}^{-1} ;{ }^{1} \mathrm{H}$ NMR $\left(500 \mathrm{MHz}, \mathrm{CDCl}_{3}\right) \delta 7.34(\mathrm{~d}, 1 \mathrm{H}, J$ = 7.7 Hz), $7.22(\mathrm{~m}, 1 \mathrm{H}), 7.14-7.11(\mathrm{~m}, 2 \mathrm{H}), 3.64(\mathrm{~s}, 2 \mathrm{H}), 2.98(\mathrm{td}, 2 \mathrm{H}, J=8.2,2.3 \mathrm{~Hz})$, $2.59(\mathrm{~m}, 2 \mathrm{H}), 2.52(\mathrm{t}, 2 \mathrm{H}, J=7.4 \mathrm{~Hz}), 1.61$ (quint, $2 \mathrm{H}, J=7.4 \mathrm{~Hz}), 1.37-1.26(\mathrm{~m}, 14 \mathrm{H})$, $0.88(\mathrm{t}, 3 \mathrm{H}, J=6.9 \mathrm{~Hz}) ;{ }^{19} \mathrm{~F}$ NMR $\left(470 \mathrm{MHz}, \mathrm{CDCl}_{3}\right) \delta-102.1(\mathrm{~s}, 1 \mathrm{~F}) ;{ }^{13} \mathrm{C} \mathrm{NMR}(126$ $\left.\mathrm{MHz}, \mathrm{CDCl}_{3}\right) \delta 159.9\left(\mathrm{~d}, J_{\mathrm{C}-\mathrm{F}}=269 \mathrm{~Hz}\right), 133.2,133.0\left(\mathrm{~d}, J_{\mathrm{C}-\mathrm{F}}=5.8 \mathrm{~Hz}\right), 127.6,126.8$, $126.4\left(\mathrm{~d}, J_{\mathrm{C}-\mathrm{F}}=2.1 \mathrm{~Hz}\right), 123.6\left(\mathrm{~d}, J_{\mathrm{C}-\mathrm{F}}=6.5 \mathrm{~Hz}\right), 111.3\left(\mathrm{~d}, J_{\mathrm{C}-\mathrm{F}}=12.5 \mathrm{~Hz}\right), 32.1\left(\mathrm{~d}, J_{\mathrm{C}-\mathrm{F}}=\right.$ 7.7 Hz), 29.70, 29.67, 29.6, 29.5, 29.4, 29.2, 28.7 (d, $\left.J_{\mathrm{C}-\mathrm{F}}=7.3 \mathrm{~Hz}\right), 25.1,24.9,24.8$ (d, $J_{\mathrm{C}-\mathrm{F}}=5.4 \mathrm{~Hz}$ ), 22.8, 14.3; HRMS-ESI calcd for $\mathrm{C}_{8} \mathrm{H}_{11} \mathrm{ClFO}[\mathrm{M}+\mathrm{H}]+335.2203$; found 335.2190 .

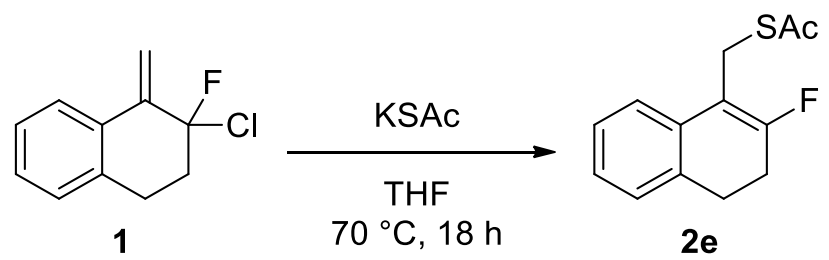

$S$-((2-Fluoro-3,4-dihydronaphthalen-1-yl)methyl) ethanethioate (2e). An argonflushed vial was charged with potassium thioacetate $(58 \mathrm{mg}, 0.509 \mathrm{mmol})$ and THF (1.5 
$\mathrm{mL})$. A solution of $1(50 \mathrm{mg}, 0.254 \mathrm{mmol})$ in THF (1 ml) was next added. The resulting mixture was stirred at $70{ }^{\circ} \mathrm{C}$ for 18 hours. Water was added and the mixture was extracted with $\mathrm{Et}_{2} \mathrm{O}(3 \mathrm{x})$. The organic layers were combined, washed with brine (1x), dried over $\mathrm{Na}_{2} \mathrm{SO}_{4}$ and concentrated in vacuo. The desired product (50 mg, 84\%) was isolated as a pale yellow oil by flash chromatography using $20 \% \mathrm{CH}_{2} \mathrm{Cl}_{2} /$ hexanes. IR $(\mathrm{ATR}, \mathrm{ZnSe}) v=3067,2944,2836,1677,1488,1426,1366,1129,962,759 \mathrm{~cm}^{-1} ;{ }^{1} \mathrm{H}$ NMR (500 MHz, CDCl $) \delta 7.20(\mathrm{~m}, 1 \mathrm{H}), 7.14-7.12(\mathrm{~m}, 3 \mathrm{H}), 4.11(\mathrm{~s}, 2 \mathrm{H}), 2.96$ (td, 2H, $J$ $=8.2,2.2 \mathrm{~Hz}), 2.57(\mathrm{~m}, 2 \mathrm{H}), 2.34(\mathrm{~s}, 3 \mathrm{H}) ;{ }^{19} \mathrm{~F}$ NMR $\left(470 \mathrm{MHz}, \mathrm{CDCl}_{3}\right) \delta-98.9(\mathrm{~s}, 1 \mathrm{~F})$; ${ }^{13} \mathrm{C}$ NMR $\left(126 \mathrm{MHz}, \mathrm{CDCl}_{3}\right) \delta 195.8,161.0\left(\mathrm{~d}, J_{\mathrm{C}-\mathrm{F}}=272 \mathrm{~Hz}\right), 132.9,132.6\left(\mathrm{~d}, J_{\mathrm{C}-\mathrm{F}}=5.4\right.$ $\mathrm{Hz}), 127.7,127.0,126.6\left(\mathrm{~d}, J_{\mathrm{C}-\mathrm{F}}=2.0 \mathrm{~Hz}\right), 123.0\left(\mathrm{~d}, J_{\mathrm{C}-\mathrm{F}}=6.7 \mathrm{~Hz}\right), 110.1\left(\mathrm{~d}, J_{\mathrm{C}-\mathrm{F}}=12.5\right.$ $\mathrm{Hz}), 30.5,28.5\left(\mathrm{~d}, J_{\mathrm{C}-\mathrm{F}}=7.2 \mathrm{~Hz}\right), 25.0\left(\mathrm{~d}, J_{\mathrm{C}-\mathrm{F}}=23.4 \mathrm{~Hz}\right), 22.5\left(\mathrm{~d}, J_{\mathrm{C}-\mathrm{F}}=6.5 \mathrm{~Hz}\right)$; HRMS-ESI calcd for $\mathrm{C}_{13} \mathrm{H}_{14} \mathrm{FOS}[\mathrm{M}+\mathrm{H}]^{+}$237.0744; found 237.0719.

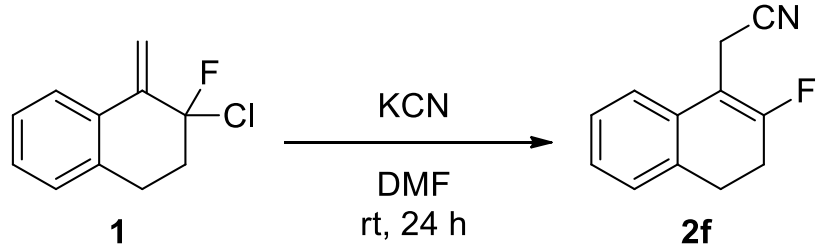

2-(2-Fluoro-3,4-dihydronaphthalen-1-yl)acetonitrile (2f). An argon-flushed vial was charged with potassium cyanide $(33 \mathrm{mg}, 0.509 \mathrm{mmol})$ and DMF $(1.5 \mathrm{~mL})$. A solution of $1(50 \mathrm{mg}, 0.254 \mathrm{mmol})$ in DMF (1 ml) was next added. The resulting mixture was stirred at ambient temperature for 24 hours. Water was added and the mixture was extracted with $\mathrm{Et}_{2} \mathrm{O}(3 \mathrm{x})$. The organic layers were combined, washed with brine (1x), dried over $\mathrm{MgSO}_{4}$ and concentrated in vacuo. The desired product (39 $\mathrm{mg}, 83 \%$ ) was isolated as a colorless oil by flash chromatography using $10 \% \mathrm{Et}_{2} \mathrm{O} /$ hexanes. IR (ATR, ZnSe) $v=$ 3063, 2947, 2252, 1686, 1488, 1368, 1155, 995, 759, $728 \mathrm{~cm}^{-1} ;{ }^{1} \mathrm{H}$ NMR $(500 \mathrm{MHz}$, $\left.\mathrm{CDCl}_{3}\right) \delta$ 7.28-7.14 (m, 4H), $3.55(\mathrm{~s}, 2 \mathrm{H}), 3.00(\mathrm{td}, 2 \mathrm{H}, J=8.3,2.4 \mathrm{~Hz}), 2.61(\mathrm{~m}, 2 \mathrm{H})$; ${ }^{19} \mathrm{~F}$ NMR $\left(470 \mathrm{MHz}, \mathrm{CDCl}_{3}\right) \delta-98.7(\mathrm{~s}, 1 \mathrm{~F}) ;{ }^{13} \mathrm{C} \mathrm{NMR}\left(126 \mathrm{MHz}, \mathrm{CDCl}_{3}\right) \delta 161.2(\mathrm{~d}$, $\left.J_{\mathrm{C}-\mathrm{F}}=273 \mathrm{~Hz}\right), 132.7,131.7\left(\mathrm{~d}, J_{\mathrm{C}-\mathrm{F}}=4.5 \mathrm{~Hz}\right), 127.9,127.22,127.21,122.6\left(\mathrm{~d}, J_{\mathrm{C}-\mathrm{F}}=6.4\right.$ $\mathrm{Hz}), 117.2\left(\mathrm{~d}, J_{\mathrm{C}-\mathrm{F}}=2.8 \mathrm{~Hz}\right), 105.1\left(\mathrm{~d}, J_{\mathrm{C}-\mathrm{F}}=12.4 \mathrm{~Hz}\right), 28.2\left(\mathrm{~d}, J_{\mathrm{C}-\mathrm{F}}=7.2 \mathrm{~Hz}\right), 24.9(\mathrm{~d}$, 
$\left.J_{\mathrm{C}-\mathrm{F}}=23.2 \mathrm{~Hz}\right), 12.7\left(\mathrm{~d}, J_{\mathrm{C}-\mathrm{F}}=7.9 \mathrm{~Hz}\right)$; HRMS-ESI calcd for $\mathrm{C}_{12} \mathrm{H}_{11} \mathrm{FN}[\mathrm{M}+\mathrm{H}]^{+}$ 188.0870; found 188.0867 .

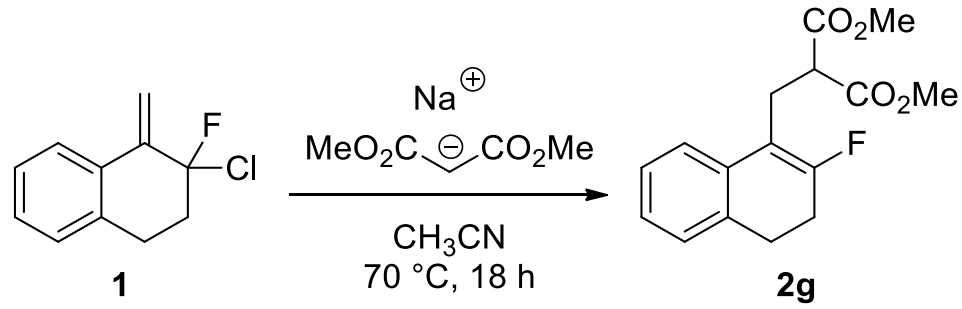

Dimethyl 2-((2-fluoro-3,4-dihydronaphthalen-1-yl)methyl)malonate (2g). An argonflushed vial was charged with $\mathrm{NaH}\left(60 \%\right.$ in mineral oil, $20 \mathrm{mg}, 0.509 \mathrm{mmol}$ ) and $\mathrm{CH}_{3} \mathrm{CN}$ $(1.5 \mathrm{~mL})$. Dimethyl malonate $(52 \mu \mathrm{L}, 0.509 \mathrm{mmol})$ was added slowly, and the resulting mixture was stirred at ambient temperature for 10 minutes. A solution of $1(50 \mathrm{mg}, 0.254$ mmol) in $\mathrm{CH}_{3} \mathrm{CN}(1 \mathrm{ml})$ was added, after which the solution was stirred at $70{ }^{\circ} \mathrm{C}$ for 18 hours. Water was added and the mixture was extracted with $\mathrm{Et}_{2} \mathrm{O}(3 \mathrm{x})$. The organic layers were combined, washed with brine (1x), dried over $\mathrm{MgSO}_{4}$ and concentrated in vacuo. The desired product $(62 \mathrm{mg}, 84 \%)$ was isolated as a colorless oil by flash chromatography using $20 \% \mathrm{Et}_{2} \mathrm{O} /$ hexanes. IR (ATR, ZnSe) $v=2952$, 2841, 1732, 1681, 1434, 1275, 1191, 1149, 1032, $760 \mathrm{~cm}^{-1} ;{ }^{1} \mathrm{H}$ NMR $\left(500 \mathrm{MHz}, \mathrm{CDCl}_{3}\right) \delta$ 7.22-7.09 (m, $4 \mathrm{H}), 3.69(\mathrm{~s}, 6 \mathrm{H}), 3.61(\mathrm{t}, 1 \mathrm{H}, J=7.7 \mathrm{~Hz}), 3.17(\mathrm{~d}, 2 \mathrm{H}, J=7.6 \mathrm{~Hz}), 2.92(\mathrm{td}, 2 \mathrm{H}, J=8.1$, $2.2 \mathrm{~Hz}), 2.52(\mathrm{~m}, 2 \mathrm{H}) ;{ }^{19} \mathrm{~F}$ NMR $\left(470 \mathrm{MHz}, \mathrm{CDCl}_{3}\right) \delta-100.7$ (s, 1F); ${ }^{13} \mathrm{C}$ NMR $(126$ $\left.\mathrm{MHz}, \mathrm{CDCl}_{3}\right) \delta 169.5,160.4\left(\mathrm{~d}, J_{\mathrm{C}-\mathrm{F}}=269 \mathrm{~Hz}\right), 133.4,133.2\left(\mathrm{~d}, J_{\mathrm{C}-\mathrm{F}}=6.3 \mathrm{~Hz}\right), 127.7$, $126.9,126.4\left(\mathrm{~d}, J_{\mathrm{C}-\mathrm{F}}=2.2 \mathrm{~Hz}\right), 122.5\left(\mathrm{~d}, J_{\mathrm{C}-\mathrm{F}}=6.6 \mathrm{~Hz}\right), 110.6\left(\mathrm{~d}, J_{\mathrm{C}-\mathrm{F}}=13.2 \mathrm{~Hz}\right), 52.7$, $50.5\left(\mathrm{~d}, J_{\mathrm{C}-\mathrm{F}}=2.3 \mathrm{~Hz}\right), 28.8\left(\mathrm{~d}, J_{\mathrm{C}-\mathrm{F}}=7.1 \mathrm{~Hz}\right), 24.9\left(\mathrm{~d}, J_{\mathrm{C}-\mathrm{F}}=24.6 \mathrm{~Hz}\right), 23.1\left(\mathrm{~d}, J_{\mathrm{C}-\mathrm{F}}=4.8\right.$ $\mathrm{Hz}$ ); HRMS-ESI calcd for $\mathrm{C}_{16} \mathrm{H}_{18} \mathrm{FO}_{4}[\mathrm{M}+\mathrm{H}]^{+}$293.1184; found 293.1179 .

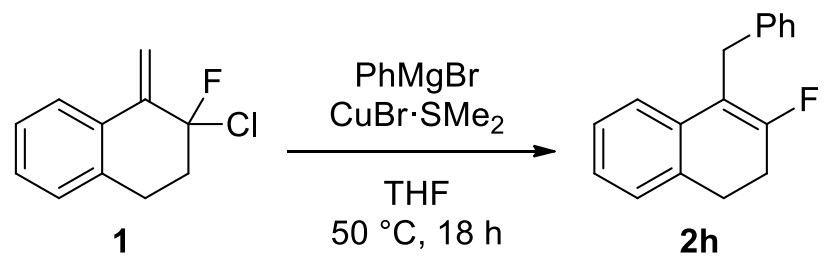


4-Benzyl-3-fluoro-1,2-dihydronaphthalene (2h). An argon-flushed vial was charged with $\mathrm{CuBr} \cdot \mathrm{SMe}_{2}(78 \mathrm{mg}, 0.381 \mathrm{mmol})$ and $\mathrm{THF}(1.5 \mathrm{~mL})$, then cooled to $0{ }^{\circ} \mathrm{C}$. Phenylmagnesium bromide ( $3 \mathrm{M}$ in $\mathrm{Et}_{2} \mathrm{O}, 127 \mu \mathrm{L}, 0.381 \mathrm{mmol}$ ) was added slowly, and the resulting mixture was stirred at $0{ }^{\circ} \mathrm{C}$ for 15 minutes. A solution of 1 (50 mg, 0.254 mmol) in THF (1 ml) was added, after which the solution was brought to $50{ }^{\circ} \mathrm{C}$ and stirred for 18 hours. Water was added and the mixture was extracted with $\mathrm{Et}_{2} \mathrm{O}(3 \mathrm{x})$. The organic layers were combined, washed with brine $(1 \mathrm{x})$, dried over $\mathrm{MgSO}_{4}$ and concentrated in vacuo. The desired product (34 mg, 75\%) was isolated as a colorless oil by flash chromatography using hexanes. Spectroscopic data was in agreement with the literature. ${ }^{3}{ }^{1} \mathrm{H}$ NMR (400 MHz, $\left.\mathrm{CDCl}_{3}\right) \delta$ 7.26-7.25 (m, 4H), $7.16(\mathrm{~m}, 1 \mathrm{H}), 7.10-7.03$ (m, 4H), 3.89 (s, 2H), $3.01(\mathrm{td}, 2 \mathrm{H}, J=8.1,2.0 \mathrm{~Hz}), 2.65(\mathrm{~m}, 2 \mathrm{H})$.

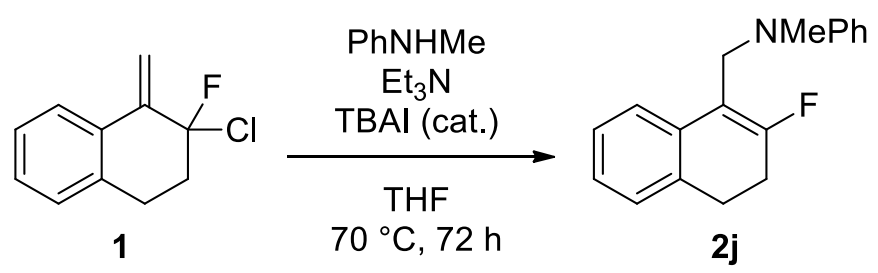

$N$-((2-Fluoro-3,4-dihydronaphthalen-1-yl)methyl)- $N$-methylaniline (2j). To an argonflushed vial charged with tetra- $n$-butylammonium iodide $(9.4 \mathrm{mg}, 25.4 \mu \mathrm{mol})$ were sequentially added THF (1.5 mL), $N$-methylaniline $(55 \mu \mathrm{L}, 0.509 \mathrm{mmol})), \mathrm{Et}_{3} \mathrm{~N}(35 \mu \mathrm{L}$, $0.254 \mathrm{mmol})$ and a solution of $1(50 \mathrm{mg}, 0.254 \mathrm{mmol})$ in THF $(1 \mathrm{~mL})$. The resulting mixture was stirred at $70{ }^{\circ} \mathrm{C}$ for 72 hours. Water was added and the mixture was extracted with $\mathrm{Et}_{2} \mathrm{O}(3 \mathrm{x})$. The organic layers were combined, washed with brine $(1 \mathrm{x})$, dried over $\mathrm{Na}_{2} \mathrm{SO}_{4}$ and concentrated in vacuo. The desired product (62 mg, 91\%) was isolated as a colorless oil by flash chromatography using hexanes. Spectroscopic data was in agreement with the literature. ${ }^{10}{ }^{1} \mathrm{H}$ NMR $\left(400 \mathrm{MHz}, \mathrm{CDCl}_{3}\right) \delta 7.32-7.25(\mathrm{~m}, 3 \mathrm{H}), 7.14-$ $7.08(\mathrm{~m}, 3 \mathrm{H}), 6.94(\mathrm{~m}, 2 \mathrm{H}), 6.79(\mathrm{~m}, 1 \mathrm{H}), 4.28(\mathrm{~d}, 2 \mathrm{H}, J=2.4 \mathrm{~Hz}), 3.01(\mathrm{td}, 2 \mathrm{H}, J=8.3$, $2.7 \mathrm{~Hz}), 2.80$ (s, 3H), 2.63 (m, 2H). 


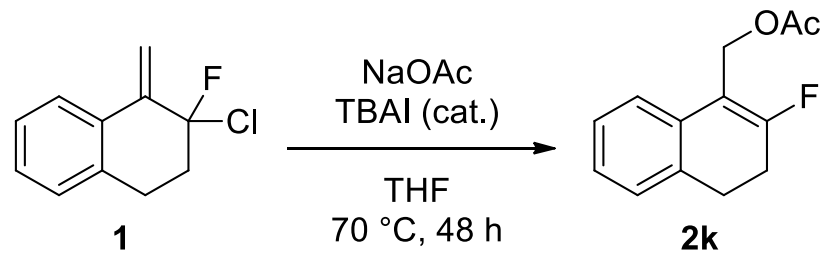

(2-Fluoro-3,4-dihydronaphthalen-1-yl)methyl acetate (2k). An argon-flushed vial was charged with sodium acetate $(42 \mathrm{mg}, 0.509 \mathrm{mmol})$, tetra- $n$-butylammonium iodide $(9.4$ $\mathrm{mg}, 25.4 \mu \mathrm{mol})$ and THF $(1.5 \mathrm{~mL})$. A solution of $1(50 \mathrm{mg}, 0.254 \mathrm{mmol})$ in THF $(1 \mathrm{ml})$ was next added. The resulting mixture was stirred at $70{ }^{\circ} \mathrm{C}$ for 48 hours. Water was added and the mixture was extracted with $\mathrm{Et}_{2} \mathrm{O}(3 \mathrm{x})$. The organic layers were combined, washed with brine $(1 \mathrm{x})$, dried over $\mathrm{MgSO}_{4}$ and concentrated in vacuo. The desired product (48 mg, 86\%) was isolated as a colorless oil using 10\% $\mathrm{Et}_{2} \mathrm{O} /$ hexanes. IR (ATR, $\mathrm{ZnSe}) v=2947,2255,1735,1682,1369,1222,1018,961,908,727 \mathrm{~cm}^{-1} ;{ }^{1} \mathrm{H}$ NMR (500 $\left.\mathrm{MHz} \mathrm{CDCl}_{3}\right) \delta$ 7.23-7.18 (m, 2H), 7.16-7.13 (m, 2H), $5.08(\mathrm{~d}, 2 \mathrm{H}, J=1.7 \mathrm{~Hz}), 3.00(\mathrm{td}$, $2 \mathrm{H}, J=8.3,2.4 \mathrm{~Hz}), 2.61(\mathrm{~m}, 2 \mathrm{H}), 2.07(\mathrm{~s}, 3 \mathrm{H}) ;{ }^{19} \mathrm{~F}$ NMR $\left(470 \mathrm{MHz}, \mathrm{CDCl}_{3}\right) \delta-98,9(\mathrm{~s}$, $1 \mathrm{~F}) ;{ }^{13} \mathrm{C} \mathrm{NMR}\left(126 \mathrm{MHz}, \mathrm{CDCl}_{3}\right) \delta 171.3,162.4\left(\mathrm{~d}, J_{\mathrm{C}-\mathrm{F}}=275 \mathrm{~Hz}\right), 132.71\left(\mathrm{~d}, J_{\mathrm{C}-\mathrm{F}}=5.2\right.$ $\mathrm{Hz}), 132.67,127.7,127.0,126.7\left(\mathrm{~d}, J_{\mathrm{C}-\mathrm{F}}=2.2 \mathrm{~Hz}\right), 123.2\left(\mathrm{~d}, J_{\mathrm{C}-\mathrm{F}}=6.7 \mathrm{~Hz}\right), 110.1\left(\mathrm{~d}, J_{\mathrm{C}-\mathrm{F}}\right.$ $=11.0 \mathrm{~Hz}), 56.3\left(\mathrm{~d}, J_{\mathrm{C}-\mathrm{F}}=8.0 \mathrm{~Hz}\right), 28.4\left(\mathrm{~d}, J_{\mathrm{C}-\mathrm{F}}=7.2 \mathrm{~Hz}\right), 25.0\left(\mathrm{~d}, J_{\mathrm{C}-\mathrm{F}}=23.9 \mathrm{~Hz}\right), 21.1$; HRMS-ESI calcd for $\mathrm{C}_{13} \mathrm{H}_{13} \mathrm{FNaO}[\mathrm{M}+\mathrm{Na}]^{+}$243.0792; found 243.0790.

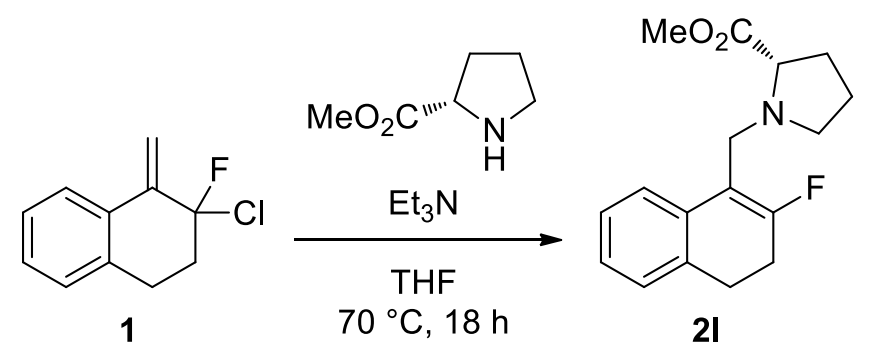

(S)-Methyl 1-((2-fluoro-3,4-dihydronaphthalen-1-yl)methyl)pyrrolidine-2carboxylate (2l). To an argon-flushed vial was added a solution of 1 (50 mg, 0.254 $\mathrm{mmol}$ ) in THF (2.5 mL), followed by L-proline methyl ester (164 mg, $1.27 \mathrm{mmol})$ ) and $\mathrm{Et}_{3} \mathrm{~N}(35 \mu \mathrm{L}, 0.254 \mathrm{mmol})$. The resulting mixture was stirred at $70{ }^{\circ} \mathrm{C}$ for 18 hours. Water was added and the mixture was extracted with $\mathrm{Et}_{2} \mathrm{O}(3 \mathrm{x})$. The organic layers were 
combined, washed with brine (1x), dried over $\mathrm{Na}_{2} \mathrm{SO}_{4}$ and concentrated in vacuo. The desired product (63 $\mathrm{mg}, 85 \%)$ was isolated as a white solid by flash chromatography using $10 \% \mathrm{Et}_{2} \mathrm{O} /$ hexanes. Spectroscopic data was in agreement with the literature. ${ }^{9}{ }^{1} \mathrm{H}$ NMR (500 MHz, $\left.\mathrm{CDCl}_{3}\right) \delta 7.67(\mathrm{~d}, 1 \mathrm{H}, J=7.7 \mathrm{~Hz}), 7.22(\mathrm{~m}, 1 \mathrm{H}), 7.11-7.07(\mathrm{~m}, 2 \mathrm{H})$, 3.72-3.59 (m, 5H), 3.24 (dd, 1H, $J=8.6,6.6 \mathrm{~Hz}), 3.10(\mathrm{~m}, 1 \mathrm{H}), 2.95$ (td, $2 \mathrm{H}, J=8.1,2.0$ $\mathrm{Hz}), 2.55(\mathrm{~m}, 2 \mathrm{H}), 2.45$ (q, 1H, $J=8.4 \mathrm{~Hz}), 2.17-2.09(\mathrm{~m}, 1 \mathrm{H}), 1.97-1.73(\mathrm{~m}, 3 \mathrm{H})$.

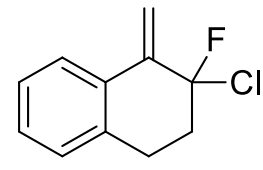

1

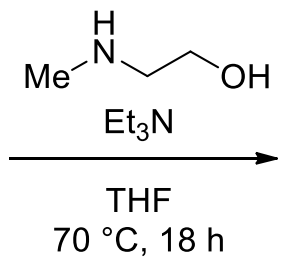

$70{ }^{\circ} \mathrm{C}, 18 \mathrm{~h}$

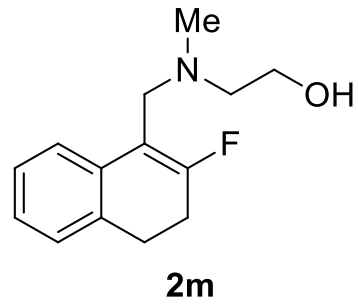

2-(((2-Fluoro-3,4-dihydronaphthalen-1-yl)methyl)(methyl)amino)ethanol (2m). To an argon-flushed vial was added a solution of 1 (50 mg, $0.254 \mathrm{mmol})$ in THF $(2.5 \mathrm{~mL})$, followed by 2-(methylamino)ethanol $\left(41 \mu \mathrm{L}, 0.509 \mathrm{mmol}^{)}\right)$and $\mathrm{Et}_{3} \mathrm{~N}(35 \mu \mathrm{L}, 0.254$ mmol). The resulting mixture was stirred at $70{ }^{\circ} \mathrm{C}$ for 18 hours. Water was added and the mixture was extracted with $\mathrm{Et}_{2} \mathrm{O}(3 \mathrm{x})$. The organic layers were combined, washed with brine (1x), dried over $\mathrm{Na}_{2} \mathrm{SO}_{4}$ and concentrated in vacuo. The desired product $(38 \mathrm{mg}$, $64 \%$ ) was isolated as a pale yellow oil by flash chromatography using $10 \%$ $\mathrm{MeOH} / \mathrm{CH}_{2} \mathrm{Cl}_{2}$. Spectroscopic data was in agreement with the literature. ${ }^{9}{ }^{1} \mathrm{H}$ NMR (400 $\left.\mathrm{MHz}, \mathrm{CDCl}_{3}\right) \delta 7.38(\mathrm{~d}, 1 \mathrm{H}, J=7.6 \mathrm{~Hz}), 7.20(\mathrm{~m}, 1 \mathrm{H}), 7.11-7.08(\mathrm{~m}, 2 \mathrm{H}), 3.61(\mathrm{t}, 2 \mathrm{H}, J$ $=5.2 \mathrm{~Hz}), 3.53(\mathrm{~d}, 2 \mathrm{H}, J=1.2 \mathrm{~Hz}), 2.98(\mathrm{td}, 2 \mathrm{H}, J=8.2,2.5 \mathrm{~Hz}), 2.89(\mathrm{bs}, 1 \mathrm{H}), 2.65-$ $2.56(\mathrm{~m}, 4 \mathrm{H}), 2.30(\mathrm{~m}, 3 \mathrm{H})$.

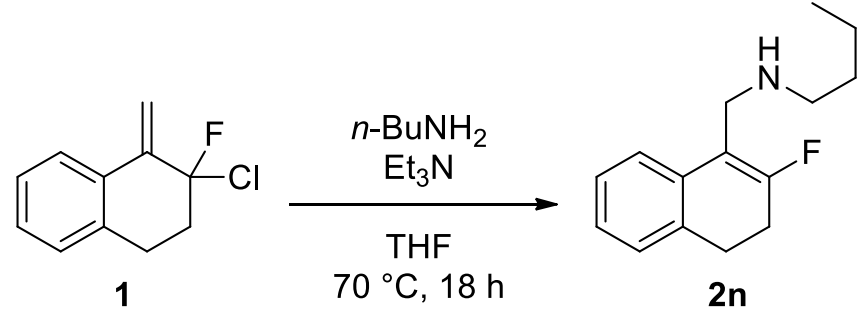


$N$-((2-Fluoro-3,4-dihydronaphthalen-1-yl)methyl)butan-1-amine (2n). To an argonflushed vial was added a solution of 1 (50 mg, $0.254 \mathrm{mmol})$ in THF (2.5 mL), followed by $n$-butylamine $(251 \mu \mathrm{L}, 2.54 \mathrm{mmol}))$ and $\mathrm{Et}_{3} \mathrm{~N}(35 \mu \mathrm{L}, 0.254 \mathrm{mmol})$. The resulting mixture was stirred at $70{ }^{\circ} \mathrm{C}$ for 18 hours. Water was added and the mixture was extracted with $\mathrm{Et}_{2} \mathrm{O}(3 \mathrm{x})$. The organic layers were combined, washed with brine $(1 \mathrm{x})$, dried over $\mathrm{Na}_{2} \mathrm{SO}_{4}$ and concentrated in vacuo. The desired product (58 mg, 97\%) was isolated as a pale yellow oil by flash chromatography using $10 \% \mathrm{MeOH} / \mathrm{CH}_{2} \mathrm{Cl}_{2}$. Spectroscopic data was in agreement with the literature. ${ }^{9}{ }^{1} \mathrm{H}$ NMR $\left(500 \mathrm{MHz}, \mathrm{CDCl}_{3}\right) \delta$ $7.30(\mathrm{~d}, 1 \mathrm{H}, J=7.7 \mathrm{~Hz}), 7.21(\mathrm{~m}, 1 \mathrm{H}), 7.11-7.09$ (m, 2H), 3.71 (d, 2H, J = 1.6 Hz), 2.96 $(\mathrm{td}, 2 \mathrm{H}, J=8.2,2.3 \mathrm{~Hz}), 2.65(\mathrm{t}, 2 \mathrm{H}, J=7.2 \mathrm{~Hz}), 2.56(\mathrm{~m}, 2 \mathrm{H}), 1.48$ (quint, $2 \mathrm{H}, J=7.3$ $\mathrm{Hz}), 1.34$ (sext, 2H, $J=7.4 \mathrm{~Hz}), 0.90$ (t, 3H, $J=7.3 \mathrm{~Hz})$.

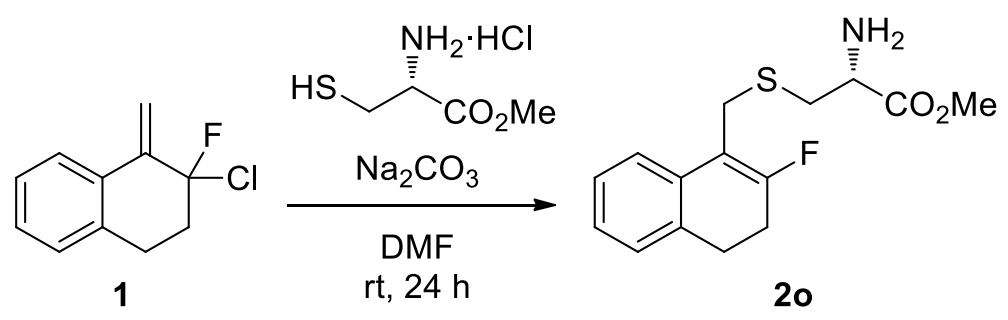

\section{(R)-Methyl}

2-amino-3-(((2-fluoro-3,4-dihydronaphthalen-1-

yl)methyl)thio)propanoate (2o). To an argon-flushed vial charged with sodium carbonate (108 mg, $1.02 \mathrm{mmol})$ was added a solution of L-cysteine methyl ester hydrochloride $(87 \mathrm{mg}, 0.509 \mathrm{mmol})$ in DMF $(1.5 \mathrm{~mL})$, and the solution was stirred at ambient temperature for 1 hour. A solution of $1(50 \mathrm{mg}, 0.254 \mathrm{mmol})$ in DMF $(1 \mathrm{~mL})$ was next added, and stirring was continued at room temperature for 24 hours. Saturated sodium carbonate was added and the mixture was extracted with $\mathrm{Et}_{2} \mathrm{O}(3 \mathrm{x})$. The organic layers were combined, washed with brine (3x), dried over $\mathrm{Na}_{2} \mathrm{SO}_{4}$ and concentrated in vacuo. The desired product $(53 \mathrm{mg}, 70 \%)$ was isolated as a yellow oil by flash chromatography using 5\% $\mathrm{MeOH} / \mathrm{CH}_{2} \mathrm{Cl}_{2}$. IR (ATR, ZnSe) $v=3375,2948,2836,1733$, 1674, 1435, 1178, 959, 828, $761 \mathrm{~cm}^{-1} ;{ }^{1} \mathrm{H} \mathrm{NMR}\left(500 \mathrm{MHz}, \mathrm{CDCl}_{3}\right) \delta 7.31$ (d, 1H, J = 7.6 Hz), 7.22 (m, 1H), 7.14-7.12 (m, 2H), 3.71-3.69 (m, 6H), 3.00-2.93 (m, 3H), 2.74 (dd, $1 \mathrm{H}, J=13.5,7.6 \mathrm{~Hz}), 2.61(\mathrm{~m}, 2 \mathrm{H}), 1.81-1.78(\mathrm{~m}, 2 \mathrm{H}) ;{ }^{19} \mathrm{~F}$ NMR $\left(470 \mathrm{MHz}, \mathrm{CDCl}_{3}\right)$ 
$\delta-101.3(\mathrm{~s}, 1 \mathrm{~F}) ;{ }^{13} \mathrm{C} \mathrm{NMR}\left(126 \mathrm{MHz}, \mathrm{CDCl}_{3}\right) \delta 174.6,160.4\left(\mathrm{~d}, J_{\mathrm{C}-\mathrm{F}}=269 \mathrm{~Hz}\right), 133.1$, $132.6\left(\mathrm{~d}, J_{\mathrm{C}-\mathrm{F}}=5.7 \mathrm{~Hz}\right), 127.6,126.8,126.6\left(\mathrm{~d}, J_{\mathrm{C}-\mathrm{F}}=2.1 \mathrm{~Hz}\right), 123.5\left(\mathrm{~d}, J_{\mathrm{C}-\mathrm{F}}=6.5 \mathrm{~Hz}\right)$, $110.7\left(\mathrm{~d}, J_{\mathrm{C}-\mathrm{F}}=12.1 \mathrm{~Hz}\right), 54.3,52.3,37.1,28.6\left(\mathrm{~d}, J_{\mathrm{C}-\mathrm{F}}=7.3 \mathrm{~Hz}\right), 25.2\left(\mathrm{~d}, J_{\mathrm{C}-\mathrm{F}}=5.6 \mathrm{~Hz}\right)$, $25.0\left(\mathrm{~d}, J_{\mathrm{C}-\mathrm{F}}=24.4 \mathrm{~Hz}\right)$; HRMS-ESI calcd for $\mathrm{C}_{15} \mathrm{H}_{19} \mathrm{FNO}_{2} \mathrm{~S}[\mathrm{M}+\mathrm{H}]^{+} 296.1115$; found 296.1127.

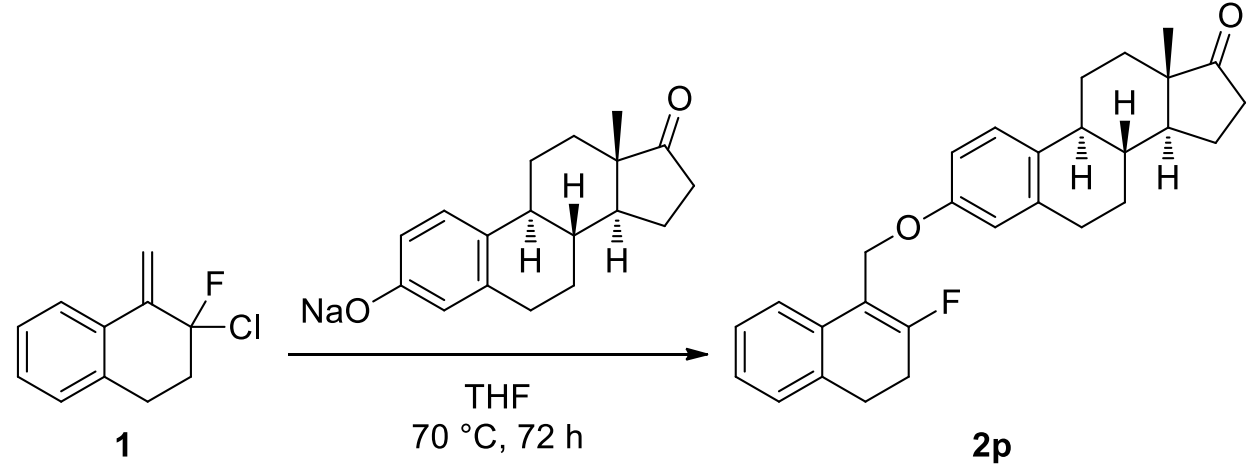

$(8 R, 9 S, 13 S, 14 S)$-3-((2-Fluoro-3,4-dihydronaphthalen-1-yl)methoxy)-13-methyl7,8,9,11,12,13,15,16-octahydro-6H-cyclopenta[a]phenanthren-17(14H)-one (2p). An argon-flushed vial was charged with $\mathrm{NaH}(60 \%$ in mineral oil, $20 \mathrm{mg}, 0.509 \mathrm{mmol})$ and THF $(0.5 \mathrm{~mL})$. A solution of estrone $(137 \mathrm{mg}, 0.509 \mathrm{mmol})$ and THF $(1 \mathrm{~mL})$ was added slowly, and the resulting mixture was stirred at ambient temperature for 20 minutes. A solution of 1 (50 mg, $0.254 \mathrm{mmol})$ in THF (1 ml) was added. The resulting mixture was stirred at $70{ }^{\circ} \mathrm{C}$ for 72 hours. Water was added and the mixture was extracted with $\mathrm{CH}_{2} \mathrm{Cl}_{2}$ (3x). The organic layers were combined, washed with brine (1x), dried over $\mathrm{Na}_{2} \mathrm{SO}_{4}$ and concentrated in vacuo. The desired product ( $85 \mathrm{mg}, 78 \%$ ) was isolated as a white solid by flash chromatography using $80 \% \mathrm{CH}_{2} \mathrm{Cl}_{2} /$ hexanes. mp $160-162{ }^{\circ} \mathrm{C}$; IR $\left(\right.$ ATR, ZnSe) $v=2923,2860,1731,1492,1388,1246,1150,1004,873,771 \mathrm{~cm}^{-1} ;{ }^{1} \mathrm{H}$ NMR $\left(500 \mathrm{MHz}, \mathrm{CDCl}_{3}\right) \delta 7.32(\mathrm{~d}, 1 \mathrm{H}, J=7.5 \mathrm{~Hz}), 7.23(\mathrm{~d}, 1 \mathrm{H}, J=8.6 \mathrm{~Hz}), 7.18-7.11$ (m, 3H), $6.83(\mathrm{dd}, 1 \mathrm{H}, J=8.5,2.4 \mathrm{~Hz}), 6.76(\mathrm{~d}, 1 \mathrm{H}, J=2.1 \mathrm{~Hz}), 4.95(\mathrm{dd}, 2 \mathrm{H}, J=1.0$ $\mathrm{Hz}), 3.01(\mathrm{td}, 2 \mathrm{H}, J=8.2,2.1 \mathrm{~Hz}), 2.63(\mathrm{~m}, 2 \mathrm{H}), 2.51(\mathrm{dd}, 1 \mathrm{H}, J=19.1,8.7 \mathrm{~Hz}), 2.41(\mathrm{~m}$, $1 \mathrm{H}), 2.27(\mathrm{~m}, 1 \mathrm{H}), 2.18-1.95(\mathrm{~m}, 4 \mathrm{H}), 1.67-1.40(\mathrm{~m}, 6 \mathrm{H}), 0.91(\mathrm{~s}, 3 \mathrm{H}) ;{ }^{19} \mathrm{~F}$ NMR $(470$ $\left.\mathrm{MHz}, \mathrm{CDCl}_{3}\right) \delta-100.1(\mathrm{~s}, 1 \mathrm{~F}) ;{ }^{13} \mathrm{C} \mathrm{NMR}\left(126 \mathrm{MHz}, \mathrm{CDCl}_{3}\right) \delta 221.1,161.7\left(\mathrm{~d}, J_{\mathrm{C}-\mathrm{F}}=273\right.$ $\mathrm{Hz}), 156.8,137.9,133.1\left(\mathrm{~d}, J_{\mathrm{C}-\mathrm{F}}=5.4 \mathrm{~Hz}\right), 132.7,132.5,127.5,127.0,126.6\left(\mathrm{~d}, J_{\mathrm{C}-\mathrm{F}}=\right.$ 
$2.0 \mathrm{~Hz}), 126.5,124.0\left(\mathrm{~d}, J_{\mathrm{C}-\mathrm{F}}=6.6 \mathrm{~Hz}\right), 115.0,112.4,111.0\left(\mathrm{~d}, J_{\mathrm{C}-\mathrm{F}}=10.8 \mathrm{~Hz}\right), 60.0(\mathrm{~d}$, $\left.J_{\mathrm{C}-\mathrm{F}}=7.5 \mathrm{~Hz}\right), 50.5,48.2,44.2,38.5,36.0,31.7,29.8,28.5\left(\mathrm{~d}, J_{\mathrm{C}-\mathrm{F}}=7.3 \mathrm{~Hz}\right), 26.7,26.1$, $25.1\left(\mathrm{~d}, J_{\mathrm{C}-\mathrm{F}}=24.1 \mathrm{~Hz}\right), 21.7,14.0$; HRMS-ESI calcd for $\mathrm{C}_{29} \mathrm{H}_{32} \mathrm{FO}_{2}[\mathrm{M}+\mathrm{H}]^{+}$431.2381; found 431.2397 .

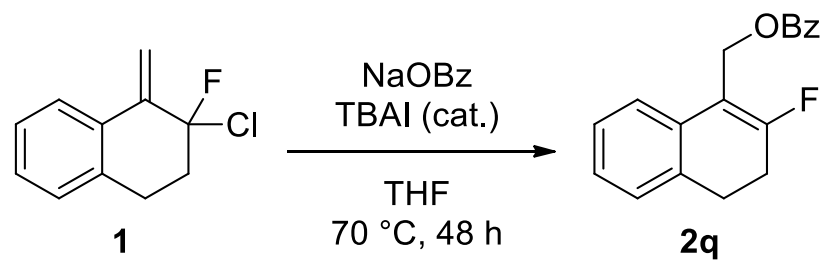

(2-Fluoro-3,4-dihydronaphthalen-1-yl)methyl benzoate (2q). An argon-flushed vial was charged with sodium benzoate $(73 \mathrm{mg}, 0.509 \mathrm{mmol})$, tetra- $n$-butylammonium iodide (9.4 mg, $25.4 \mu \mathrm{mol})$ and THF (1.5 mL). A solution of 1 (50 mg, $0.254 \mathrm{mmol})$ in THF (1 ml) was next added. The resulting mixture was stirred at $70{ }^{\circ} \mathrm{C}$ for 48 hours. Water was added and the mixture was extracted with $\mathrm{Et}_{2} \mathrm{O}(3 \mathrm{x})$. The organic layers were combined, washed with brine $(1 \mathrm{x})$, dried over $\mathrm{MgSO}_{4}$ and concentrated in vacuo. The desired product (63 mg, 88\%) was isolated as a colorless oil using 10\% $\mathrm{Et}_{2} \mathrm{O} /$ hexanes. mp 79-81 ${ }^{\circ} \mathrm{C}$; IR (ATR, ZnSe) v = 3057, 2949, 1716, 1680, 1450, 1264, 1097, 1024, 943, 769, 705 $\mathrm{cm}^{-1} ;{ }^{1} \mathrm{H}$ NMR $\left(500 \mathrm{MHz}, \mathrm{CDCl}_{3}\right) \delta 8.02(\mathrm{~d}, 2 \mathrm{H}, J=7.4 \mathrm{~Hz}), 7.53(\mathrm{t}, 1 \mathrm{H}, J=7.4 \mathrm{~Hz})$, $7.40(\mathrm{t}, 2 \mathrm{H}, J=7.7 \mathrm{~Hz}), 7.32(\mathrm{~d}, 1 \mathrm{H}, J=7.6 \mathrm{~Hz}), 7.20(\mathrm{~m}, 1 \mathrm{H}), 7.14-7.13(\mathrm{~m}, 2 \mathrm{H}), 5.33$ $(\mathrm{s}, 2 \mathrm{H}), 3.03(\mathrm{td}, 2 \mathrm{H}, J=8.2,2.1 \mathrm{~Hz}), 2.64(\mathrm{~m}, 2 \mathrm{H}) ;{ }^{19} \mathrm{~F}$ NMR $\left(470 \mathrm{MHz}, \mathrm{CDCl}_{3}\right) \delta-98.7$ $(\mathrm{s}, 1 \mathrm{~F}) ;{ }^{13} \mathrm{C} \mathrm{NMR}\left(126 \mathrm{MHz}, \mathrm{CDCl}_{3}\right) \delta 166.7,162.5\left(\mathrm{~d}, J_{\mathrm{C}-\mathrm{F}}=275 \mathrm{~Hz}\right), 133.1,132.9(\mathrm{~d}$, $\left.J_{\mathrm{C}-\mathrm{F}}=5.2 \mathrm{~Hz}\right), 132.7,130.2,129.9,128.5,127.7,127.1,126.7\left(\mathrm{~d}, J_{\mathrm{C}-\mathrm{F}}=2.1 \mathrm{~Hz}\right), 123.4$ $\left(\mathrm{d}, J_{\mathrm{C}-\mathrm{F}}=6.6 \mathrm{~Hz}\right), 110.3\left(\mathrm{~d}, J_{\mathrm{C}-\mathrm{F}}=10.9 \mathrm{~Hz}\right), 56.9\left(\mathrm{~d}, J_{\mathrm{C}-\mathrm{F}}=8.0 \mathrm{~Hz}\right), 28.4\left(\mathrm{~d}, J_{\mathrm{C}-\mathrm{F}}=7.2\right.$ $\mathrm{Hz}), 25.1\left(\mathrm{~d}, J_{\mathrm{C}-\mathrm{F}}=23.9 \mathrm{~Hz}\right)$; HRMS-ESI calcd for $\mathrm{C}_{18} \mathrm{H}_{15} \mathrm{FNaO}_{2}[\mathrm{M}+\mathrm{H}]^{+} 305.0948$; found 305.0953 .

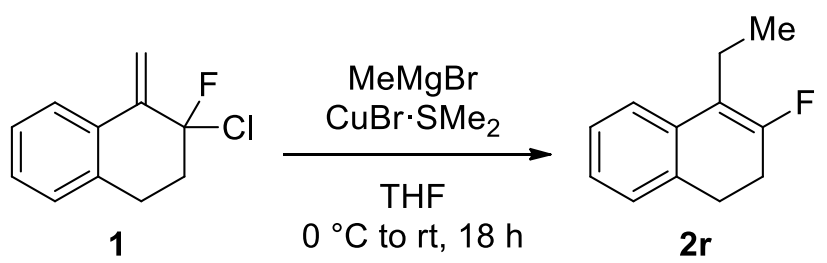


4-Ethyl-3-fluoro-1,2-dihydronaphthalene (2r). An argon-flushed vial was charged with $\mathrm{CuBr} \cdot \mathrm{SMe}_{2}(78 \mathrm{mg}, 0.381 \mathrm{mmol})$ and $\mathrm{THF}(1.5 \mathrm{~mL})$, then cooled to $0{ }^{\circ} \mathrm{C}$. Methylmagnesium bromide ( $3 \mathrm{M}$ in $\mathrm{Et}_{2} \mathrm{O}, 127 \mu \mathrm{L}, 0.381 \mathrm{mmol}$ ) was added slowly, and the resulting mixture was stirred at $0{ }^{\circ} \mathrm{C}$ for 15 minutes. A solution of $\mathbf{1}(50 \mathrm{mg}, 0.254$ mmol) in THF ( $1 \mathrm{ml})$ was added, after which the solution was brought to ambient temperature over 18 hours. Water was added and the mixture was extracted with $\mathrm{Et}_{2} \mathrm{O}$ (3x). The organic layers were combined, washed with brine (1x), dried over $\mathrm{MgSO}_{4}$ and concentrated in vacuo. The desired product (34 $\mathrm{mg}, 75 \%$ ) was isolated as a colorless oil by flash chromatography using hexanes. Spectroscopic data was in agreement with the literature. ${ }^{3}{ }^{1} \mathrm{H}$ NMR $\left(500 \mathrm{MHz}, \mathrm{CDCl}_{3}\right) \delta$ 7.22-7.17 (m, 2H), 7.11-7.08 (m, 2H), 2.94 (td, $2 \mathrm{H}, J=8.2,2.4 \mathrm{~Hz}), 2.55-2.51(\mathrm{~m}, 4 \mathrm{H}), 1.12(\mathrm{t}, 3 \mathrm{H}, J=7.5 \mathrm{~Hz})$.

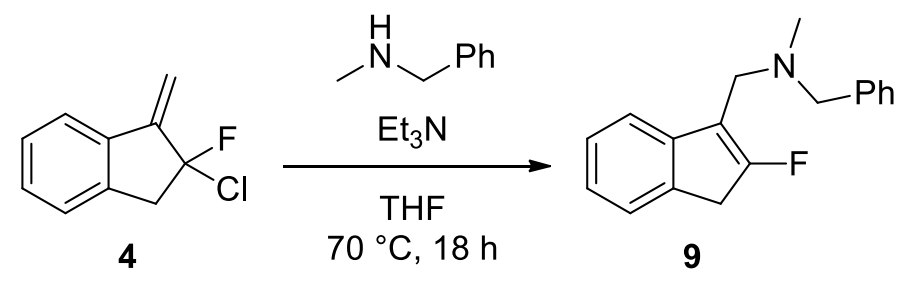

$N$-Benzyl-1-(2-fluoro-1H-inden-3-yl)- $N$-methylmethanamine (9). To an argon-flushed vial was added a solution of $4(47 \mathrm{mg}, 0.257 \mathrm{mmol})$ in THF $(2.6 \mathrm{~mL})$, followed by $N$ benzylmethylamine $(66 \mu \mathrm{L}, 0.515 \mathrm{mmol}))$ and $\mathrm{Et}_{3} \mathrm{~N}(36 \mu \mathrm{L}, 0.257 \mathrm{mmol})$. The resulting mixture was stirred at $70{ }^{\circ} \mathrm{C}$ for 18 hours. Water was added and the mixture was extracted with $\mathrm{Et}_{2} \mathrm{O}$ (3x). The organic layers were combined, washed with brine (1x), dried over $\mathrm{MgSO}_{4}$ and concentrated in vacuo. The desired product (54 $\mathrm{mg}, 78 \%$ ) was isolated as a yellow oil by flash chromatography using $5 \% \mathrm{Et}_{2} \mathrm{O} /$ hexanes. Spectroscopic data was in agreement with the literature. ${ }^{9}{ }^{1} \mathrm{H}$ NMR $\left(500 \mathrm{MHz}, \mathrm{CDCl}_{3}\right) \delta 7.44(\mathrm{~d}, 1 \mathrm{H}, J=$ $7.5 \mathrm{~Hz}$ ), 7.35-7.23 (m, 7H), 7.15 (t, 1H, $J=7.2 \mathrm{~Hz}), 3.55$ (s, 2H), 3.48 (s, 2H), 3.45 (s, $2 \mathrm{H}), 2.21(\mathrm{~s}, 3 \mathrm{H})$. 


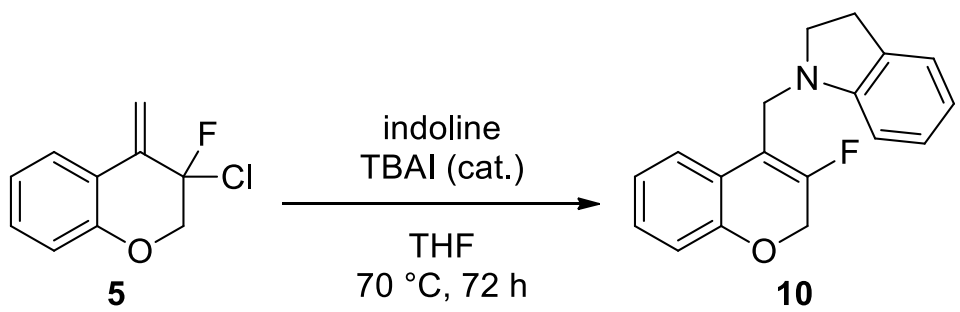

1-((3-Fluoro-2H-chromen-4-yl)methyl)indoline (10). To an argon-flushed vial charged with tetra- $n$-butylammonium iodide $(9.3 \mathrm{mg}, 25.2 \mu \mathrm{mol})$ was added THF $(1.5 \mathrm{~mL})$ and a solution of $5(50 \mathrm{mg}, 0.252 \mathrm{mmol})$ in THF $(1 \mathrm{~mL})$. The resulting mixture was stirred at $70{ }^{\circ} \mathrm{C}$ for 72 hours. Water was added and the mixture was extracted with $\mathrm{Et}_{2} \mathrm{O}(3 \mathrm{x})$. The organic layers were combined, washed with brine $(1 \mathrm{x})$, dried over $\mathrm{Na}_{2} \mathrm{SO}_{4}$ and concentrated in vacuo. The desired product (43 mg, 61\%) was isolated as a colorless oil sensitive to light and air by flash chromatography using $2 \% \mathrm{Et}_{2} \mathrm{O} /$ hexanes. IR (ATR, $\mathrm{ZnSe}) v=3042,2922,2845,1702,1605,1485,1235,1154,1041,743 \mathrm{~cm}^{-1} ;{ }^{1} \mathrm{H}$ NMR $\left(500 \mathrm{MHz} \mathrm{CDCl}_{3}\right) \delta 7.38(\mathrm{dd}, 1 \mathrm{H}, J=7.6,0.9 \mathrm{~Hz}), 7.15-7.09(\mathrm{~m}, 3 \mathrm{H}), 6.91(\mathrm{t}, 1 \mathrm{H}, J=$ $7.5 \mathrm{~Hz}), 6.85(\mathrm{~d}, 1 \mathrm{H}, J=8.0 \mathrm{~Hz}), 6.72(\mathrm{t}, 1 \mathrm{H}, J=7.4 \mathrm{~Hz}), 6.68(\mathrm{~d}, 1 \mathrm{H}, J=7.8 \mathrm{~Hz}), 4.84$ $(\mathrm{s}, 2 \mathrm{H}), 4.07(\mathrm{~s}, 2 \mathrm{H}), 3.28(\mathrm{t}, 2 \mathrm{H}, J=8.2 \mathrm{~Hz}), 2.90(\mathrm{t}, 2 \mathrm{H}, J=8.2 \mathrm{~Hz}) ;{ }^{19} \mathrm{~F}$ NMR (470 $\left.\mathrm{MHz} \mathrm{CDCl}_{3}\right) \delta-118.7$ (s, 1F); ${ }^{13} \mathrm{C} \mathrm{NMR}\left(126 \mathrm{MHz}, \mathrm{CDCl}_{3}\right) \delta 152.30\left(\mathrm{~d}, J_{\mathrm{C}-\mathrm{F}}=271 \mathrm{~Hz}\right)$, 152.27, 151.7, 130.7, $128.3\left(\mathrm{~d}, J_{\mathrm{C}-\mathrm{F}}=2.2 \mathrm{~Hz}\right), 127.5,124.8,124.5\left(\mathrm{~d}, J_{\mathrm{C}-\mathrm{F}}=6.4 \mathrm{~Hz}\right)$, $122.3,121.7\left(\mathrm{~d}, J_{\mathrm{C}-\mathrm{F}}=3.9 \mathrm{~Hz}\right), 118.4,115.8,108.8\left(\mathrm{~d}, J_{\mathrm{C}-\mathrm{F}}=8.3 \mathrm{~Hz}\right), 107.2,63.6\left(\mathrm{~d}, J_{\mathrm{C}-\mathrm{F}}\right.$ $=37.2 \mathrm{~Hz}), 53.3,42.1\left(\mathrm{~d}, J_{\mathrm{C}-\mathrm{F}}=3.3 \mathrm{~Hz}\right), 28.5$; HRMS-ESI calcd for $\mathrm{C}_{18} \mathrm{H}_{19} \mathrm{KNO}[\mathrm{M}+\mathrm{K}]^{+}$ 304.1098; found 304.1103.

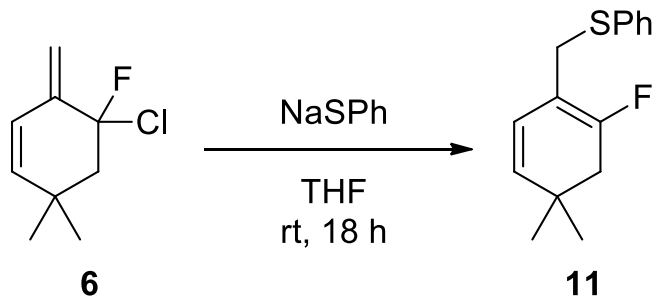

((2-Fluoro-4,4-dimethylcyclohexa-1,5-dien-1-yl)methyl)(phenyl)sulfane (11). An argon-flushed vial was charged with $\mathrm{NaH}(60 \%$ in mineral oil, $22 \mathrm{mg}, 0.538 \mathrm{mmol})$ and THF (1.6 mL). Thiophenol $(55 \mu \mathrm{L}, 0.538 \mathrm{mmol})$ was added slowly, and the resulting 
mixture was stirred at ambient temperature for 5 minutes. A solution of $\mathbf{1}$ (50 mg, 0.254 mmol) in THF $(1.1 \mathrm{ml})$ was added. The resulting mixture was stirred at ambient temperature for 18 hours. $\mathrm{NaOH}(0.1 \mathrm{M})$ was added and the mixture was extracted with $\mathrm{Et}_{2} \mathrm{O}(2 \mathrm{x})$. The organic layers were combined, washed with brine (1x), dried over $\mathrm{MgSO}_{4}$ and concentrated in vacuo. The desired product (41 $\mathrm{mg}, 61 \%$ ) was isolated as a colorless oil by flash chromatography using hexanes. IR (ATR, ZnSe) $v=3060,2957,2886,1685$, 1582, 1480, 1337, 892, 735, $687 \mathrm{~cm}^{-1} ;{ }^{1} \mathrm{H}$ NMR (500 MHz, $\left.\mathrm{CDCl}_{3}\right) \delta 7.36(\mathrm{~d}, 2 \mathrm{H}, J=7.8$ $\mathrm{Hz}), 7.26(\mathrm{t}, 2 \mathrm{H}, J=7.5 \mathrm{~Hz}), 7.18(\mathrm{t}, 2 \mathrm{H}, J=7.2 \mathrm{~Hz}), 5.76(\mathrm{t}, 1 \mathrm{H}, J=8.6 \mathrm{~Hz}), 5.34(\mathrm{~d}$, $1 \mathrm{H}, J=9.5 \mathrm{~Hz}), 3.64(\mathrm{~s}, 2 \mathrm{H}), 2.21(\mathrm{~d}, 2 \mathrm{H}, J=3.4 \mathrm{~Hz}), 0.96(\mathrm{~s}, 6 \mathrm{H}) ;{ }^{19} \mathrm{~F}$ NMR $(470 \mathrm{MHz}$, $\left.\mathrm{CDCl}_{3}\right) \delta-108.3(\mathrm{~s}, 1 \mathrm{~F}) ;{ }^{13} \mathrm{C} \mathrm{NMR}\left(126 \mathrm{MHz}, \mathrm{CDCl}_{3}\right) \delta 157.5\left(\mathrm{~d}, J_{\mathrm{C}-\mathrm{F}}=270 \mathrm{~Hz}\right), 135.6$, $133.9\left(\mathrm{~d}, J_{\mathrm{C}-\mathrm{F}}=3.4 \mathrm{~Hz}\right), 130.7,128.8,126.6,122.1\left(\mathrm{~d}, J_{\mathrm{C}-\mathrm{F}}=3.8 \mathrm{~Hz}\right), 107.5\left(\mathrm{~d}, J_{\mathrm{C}-\mathrm{F}}=\right.$ $11.7 \mathrm{~Hz}), 39.1\left(\mathrm{~d}, J_{\mathrm{C}-\mathrm{F}}=19.9 \mathrm{~Hz}\right), 34.7\left(\mathrm{~d}, J_{\mathrm{C}-\mathrm{F}}=6.5 \mathrm{~Hz}\right), 30.5\left(\mathrm{~d}, J_{\mathrm{C}-\mathrm{F}}=3.6 \mathrm{~Hz}\right), 28.3(\mathrm{~d}$, $J_{\mathrm{C}-\mathrm{F}}=1.5 \mathrm{~Hz}$ ); HRMS-ESI calcd for $\mathrm{C}_{30} \mathrm{H}_{34} \mathrm{~F}_{2} \mathrm{NaS}_{2}[2 \mathrm{M}+\mathrm{Na}]^{+}$519.1962; found 519.1935.

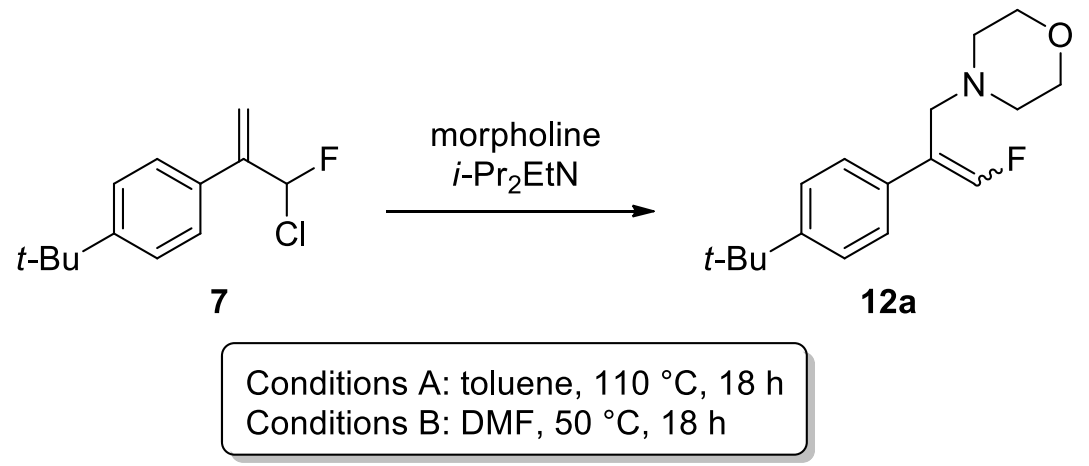

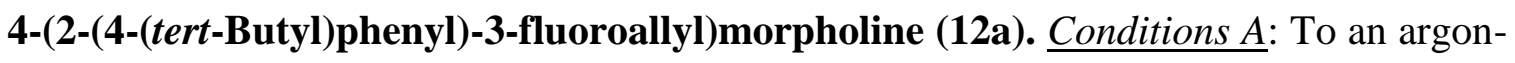
flushed vial was added a solution of $7(50 \mathrm{mg}, 0.221 \mathrm{mmol})$ in toluene $(1.1 \mathrm{~mL})$, followed by morpholine $(38 \mu \mathrm{L}, 0.441 \mathrm{mmol}))$ and diisopropylethylamine $(58 \mu \mathrm{L}, 0.331 \mathrm{mmol})$. The resulting mixture was stirred at $110{ }^{\circ} \mathrm{C}$ for 18 hours. Water was added and the mixture was extracted with $\mathrm{Et}_{2} \mathrm{O}(3 \mathrm{x})$. The organic layers were combined, washed with brine (1x), dried over $\mathrm{Na}_{2} \mathrm{SO}_{4}$ and concentrated in vacuo. The desired product $(55 \mathrm{mg}$, $89 \%, E / Z=29: 71)$ was isolated as a white solid by flash chromatography using $20 \%$ $\mathrm{Et}_{2} \mathrm{O} /$ hexanes. Conditions B: To an argon-flushed vial was added a solution of 7 (50 $\mathrm{mg}$, $0.221 \mathrm{mmol})$ in DMF $(1.1 \mathrm{~mL})$, followed by morpholine $(38 \mu \mathrm{L}, 0.441 \mathrm{mmol}))$ and 
diisopropylethylamine $(58 \mu \mathrm{L}, 0.331 \mathrm{mmol})$. The resulting mixture was stirred at $50{ }^{\circ} \mathrm{C}$ for 18 hours. Water was added and the mixture was extracted with $\mathrm{Et}_{2} \mathrm{O}(3 \mathrm{x})$. The organic layers were combined, washed with brine $(1 \mathrm{x})$, dried over $\mathrm{Na}_{2} \mathrm{SO}_{4}$ and concentrated in vacuo. The desired product $(55 \mathrm{mg}, 90 \%, E / Z=61: 39)$ was isolated as a white solid by flash chromatography using $20 \% \mathrm{Et}_{2} \mathrm{O} /$ hexanes. (E)-12a: mp 66-69 ${ }^{\circ} \mathrm{C}$; IR (ATR, ZnSe) $v=2953,2842,1654,1452,1267,1115,1085,1070,1000,850 \mathrm{~cm}^{-1} ;{ }^{1} \mathrm{H}$ NMR (500 $\left.\mathrm{MHz} \mathrm{CDCl}_{3}\right) \delta 7.56(\mathrm{~d}, 2 \mathrm{H}, J=8.1 \mathrm{~Hz}), 7.38(\mathrm{~d}, 2 \mathrm{H}, J=8.2 \mathrm{~Hz}), 6.71(\mathrm{~d}, 1 \mathrm{H}, J=83.8$ $\mathrm{Hz}), 3.68(\mathrm{t}, 4 \mathrm{H}, 4.4 \mathrm{~Hz}), 3.13(\mathrm{~d}, 2 \mathrm{H}, J=3.6 \mathrm{~Hz}), 2.46(\mathrm{~m}, 4 \mathrm{H}), 1.33(\mathrm{~s}, 9 \mathrm{H}) ;{ }^{19} \mathrm{~F} \mathrm{NMR}$ $\left(470 \mathrm{MHz}, \mathrm{CDCl}_{3}\right) \delta-127.2(\mathrm{~d}, J=83.9 \mathrm{~Hz}) ;{ }^{13} \mathrm{C} \mathrm{NMR}\left(126 \mathrm{MHz}, \mathrm{CDCl}_{3}\right) \delta 150.6$, $147.3\left(\mathrm{~d}, J_{\mathrm{C}-\mathrm{F}}=268 \mathrm{~Hz}\right), 131.6,128.0\left(\mathrm{~d}, J_{\mathrm{C}-\mathrm{F}}=5.2 \mathrm{~Hz}\right), 125.2,117.7,67.1,59.3\left(\mathrm{~d}, J_{\mathrm{C}-\mathrm{F}}\right.$ $=8.5 \mathrm{~Hz}$ ), 53.4, 34.7, 31.4; HRMS-ESI calcd for $\mathrm{C}_{17} \mathrm{H}_{25} \mathrm{FNO}[\mathrm{M}+\mathrm{H}]^{+}$278.1915; found 278.1908. (Z)-12a: $\mathrm{mp} 61-64{ }^{\circ} \mathrm{C}$; IR (ATR, ZnSe) $v=2952,2843,2807,1650,1456$, 1366, 1265, 1108, 1007, $832 \mathrm{~cm}^{-1} ;{ }^{1} \mathrm{H}$ NMR (500 MHz, $\left.\mathrm{CDCl}_{3}\right) \delta 7.40$ (d, 2H, J=8.3 $\mathrm{Hz}), 7.35(\mathrm{~d}, 2 \mathrm{H}, J=8.3 \mathrm{~Hz}), 6.95(\mathrm{~d}, 1 \mathrm{H}, \mathrm{J}=85.1 \mathrm{~Hz}), 3.67$ (t, 4H, J=4.4 Hz), 3.41 (d, $2 \mathrm{H}, J=3.3 \mathrm{~Hz}), 2.49(\mathrm{~m}, 4 \mathrm{H}), 1.32(\mathrm{~s}, 9 \mathrm{H}) ;{ }^{19} \mathrm{~F}$ NMR $\left(470 \mathrm{MHz}, \mathrm{CDCl}_{3}\right) \delta-128.6(\mathrm{~d}, 1 \mathrm{~F}$, $J=85.1 \mathrm{~Hz}) ;{ }^{13} \mathrm{C}$ NMR $\left(126 \mathrm{MHz}, \mathrm{CDCl}_{3}\right) \delta 150.7,148.3\left(\mathrm{~d}, J_{\mathrm{C}-\mathrm{F}}=263 \mathrm{~Hz}\right), 133.5(\mathrm{~d}$, $\left.J_{\mathrm{C}-\mathrm{F}}=8.0 \mathrm{~Hz}\right), 126.4\left(\mathrm{~d}, J_{\mathrm{C}-\mathrm{F}}=3.0 \mathrm{~Hz}\right), 125.6,120.3\left(\mathrm{~d}, J_{\mathrm{C}-\mathrm{F}}=6.5 \mathrm{~Hz}\right), 67.2,54.1\left(\mathrm{~d}, J_{\mathrm{C}-\mathrm{F}}\right.$ $=3.5 \mathrm{~Hz}$ ), 53.4, 34.6, 31.4; HRMS-ESI calcd for $\mathrm{C}_{17} \mathrm{H}_{25} \mathrm{FNO}[\mathrm{M}+\mathrm{H}]^{+}$278.1915; found 278.1913 .

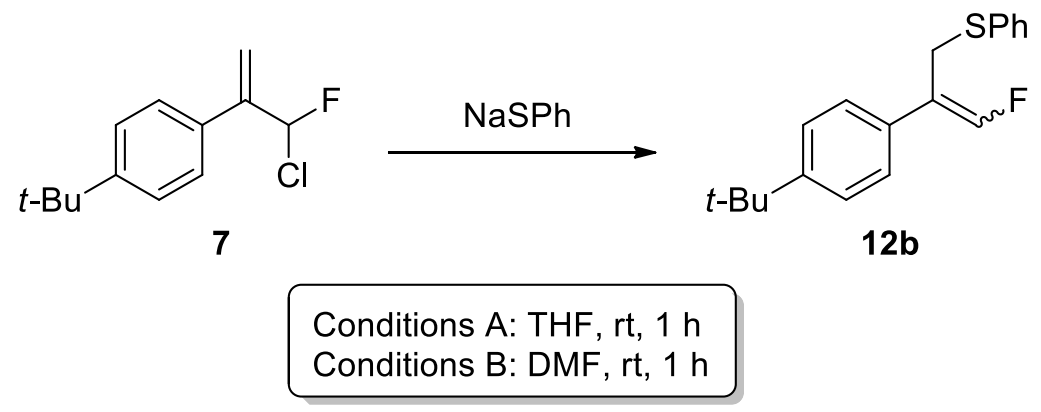

(2-(4-(tert-Butyl)phenyl)-3-fluoroallyl)(phenyl)sulfane (12b). Conditions A: An argonflushed vial was charged with $\mathrm{NaH}(60 \%$ in mineral oil, $18 \mathrm{mg}, 0.441 \mathrm{mmol})$ and THF (1.2 mL). Thiophenol (45 $\mu \mathrm{L}, 0.441 \mathrm{mmol})$ was added slowly, and the resulting mixture was stirred at ambient temperature for 5 minutes. A solution of 7 (50 mg, $0.221 \mathrm{mmol})$ in 
THF (1 ml) was added. The resulting mixture was stirred at ambient temperature for 1 hour. $\mathrm{NaOH}(0.1 \mathrm{M})$ was added and the mixture was extracted with $\mathrm{Et}_{2} \mathrm{O}(3 \mathrm{x})$. The organic layers were combined, washed with brine $(1 \mathrm{x})$, dried over $\mathrm{MgSO}_{4}$ and concentrated in vacuo. The desired product $(62 \mathrm{mg}, 94 \%, E / Z=36: 64)$ was isolated as a colorless oil by flash chromatography using $2 \% \mathrm{Et}_{2} \mathrm{O} /$ hexanes. Conditions B: An argonflushed vial was charged with $\mathrm{NaH}(60 \%$ in mineral oil, $18 \mathrm{mg}, 0.441 \mathrm{mmol})$ and DMF $(1.2 \mathrm{~mL})$. Thiophenol (45 $\mu \mathrm{L}, 0.441 \mathrm{mmol})$ was added slowly, and the resulting mixture was stirred at ambient temperature for 5 minutes. A solution of $7(50 \mathrm{mg}, 0.221 \mathrm{mmol})$ in THF (1 ml) was added. The resulting mixture was stirred at ambient temperature for 1 hour. $\mathrm{NaOH}(0.1 \mathrm{M})$ was added and the mixture was extracted with $\mathrm{Et}_{2} \mathrm{O}(3 \mathrm{x})$. The organic layers were combined, washed with brine $(1 \mathrm{x})$, dried over $\mathrm{MgSO}_{4}$ and concentrated in vacuo. The desired product (61 mg, 92\%, E/Z =62:38) was isolated as a colorless oil by flash chromatography using $2 \% \mathrm{Et}_{2} \mathrm{O} /$ hexanes. IR (ATR, ZnSe) $v=3077$, 2961, 2867, 1652, 1479, 1105, 986, 821, 736, $689 \mathrm{~cm}^{-1} ;{ }^{1} \mathrm{H}$ NMR (500 MHz, $\left.\mathrm{CDCl}_{3}\right) \delta$ 7.44-7.19 (m, 9H), 6.80 (d, 1H ( $Z$ isomer), $J=84.1 \mathrm{~Hz}), 6.54$ (d, $1 \mathrm{H}$ ( $E$ isomer), $J=82.8$ $\mathrm{Hz}), 4.01(\mathrm{~s}, 2 \mathrm{H}(\mathrm{Z}$ isomer $)), 3.74(\mathrm{~d}, 2 \mathrm{H}$ ( $E$ isomer $), J=3.4 \mathrm{~Hz}), 1.33-1.32(\mathrm{~m}, 9 \mathrm{H}) ;{ }^{19} \mathrm{~F}$ NMR (470 MHz, $\left.\mathrm{CDCl}_{3}\right) \delta-127.3-127.5(\mathrm{~m}, 1 \mathrm{~F}) ;{ }^{13} \mathrm{C} \mathrm{NMR}\left(126 \mathrm{MHz}, \mathrm{CDCl}_{3}\right) \delta 151.1$ $\left(\mathrm{d}, J_{\mathrm{C}-\mathrm{F}}=0.7 \mathrm{~Hz}\right), 151.0\left(\mathrm{~d}, J_{\mathrm{C}-\mathrm{F}}=0.6 \mathrm{~Hz}\right), 147.3\left(\mathrm{~d}, J_{\mathrm{C}-\mathrm{F}}=265 \mathrm{~Hz}\right), 146.4\left(\mathrm{~d}, J_{\mathrm{C}-\mathrm{F}}=268\right.$ $\mathrm{Hz}), 135.8,135.5,131.9\left(\mathrm{~d}, J_{\mathrm{C}-\mathrm{F}}=7.5 \mathrm{~Hz}\right), 131.7,131.0,130.7,129.1,128.9,128.1(\mathrm{~d}$, $\left.J_{\mathrm{C}-\mathrm{F}}=4.7 \mathrm{~Hz}\right), 127.2,126.8,126.7\left(\mathrm{~d}, J_{\mathrm{C}-\mathrm{F}}=3.0 \mathrm{~Hz}\right), 125.7,125.5,120.9\left(\mathrm{~d}, J_{\mathrm{C}-\mathrm{F}}=7.7\right.$ $\mathrm{Hz}), 118.3\left(\mathrm{~d}, J_{\mathrm{C}-\mathrm{F}}=3.6 \mathrm{~Hz}\right), 36.3\left(\mathrm{~d}, J_{\mathrm{C}-\mathrm{F}}=8.1 \mathrm{~Hz}\right), 34.75,34.70,31.4,31.3\left(\mathrm{~d}, J_{\mathrm{C}-\mathrm{F}}=\right.$ $4.8 \mathrm{~Hz}$ ); HRMS-ESI calcd for $\mathrm{C}_{19} \mathrm{H}_{22} \mathrm{FS}[\mathrm{M}+\mathrm{H}]^{+} 301.1421$; found 301.1425 .
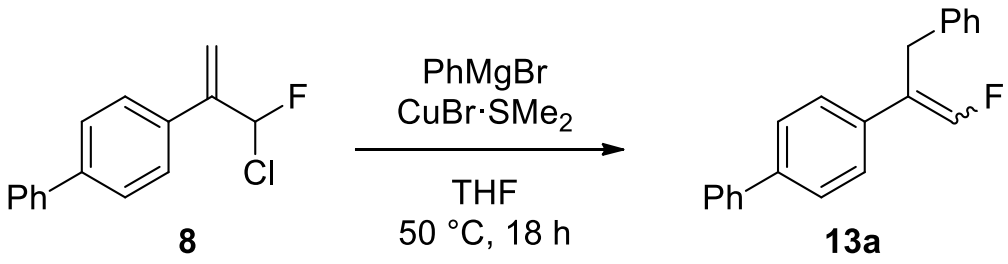

4-(1-Fluoro-3-phenylprop-1-en-2-yl)-1,1'-biphenyl (13a). An argon-flushed vial was charged with $\mathrm{CuBr} \cdot \mathrm{SMe}_{2}(62 \mathrm{mg}, 0.304 \mathrm{mmol})$ and $\mathrm{THF}(1.0 \mathrm{~mL})$, then cooled to $0{ }^{\circ} \mathrm{C}$. Phenylmagnesium bromide ( $3 \mathrm{M}$ in $\left.\mathrm{Et}_{2} \mathrm{O}, 101 \mu \mathrm{L}, 0.304 \mathrm{mmol}\right)$ was added slowly, and 
the resulting mixture was stirred at $0{ }^{\circ} \mathrm{C}$ for 15 minutes. A solution of $\mathbf{8}(50 \mathrm{mg}, 0.203$ mmol) in THF (1 ml) was added, after which the solution was brought to $50{ }^{\circ} \mathrm{C}$ and stirred for 18 hours. Water was added and the mixture was extracted with $\mathrm{Et}_{2} \mathrm{O}(3 \mathrm{x})$. The organic layers were combined, washed with brine $(1 \mathrm{x})$, dried over $\mathrm{MgSO}_{4}$ and concentrated in vacuo. The desired product (41 mg, 69\%, $E / Z=50: 50$ ) was isolated as a white solid by flash chromatography using hexanes. mp 69-72 ${ }^{\circ} \mathrm{C}$; IR (ATR, ZnSe) $v=$ 3030, 2920, 2852, 1643, 1486, 1121, 1087, 999, 830, $689 \mathrm{~cm}^{-1} ;{ }^{1} \mathrm{H}$ NMR (500 MHz, $\left.\mathrm{CDCl}_{3}\right) \delta 7.57-7.17(\mathrm{~m}, 14 \mathrm{H}), 7.07(\mathrm{~d}, 1 \mathrm{H}(E$ isomer $), J=85.0 \mathrm{~Hz}), 6.71(\mathrm{~d}, 1 \mathrm{H},(Z$ isomer), $J=83.9 \mathrm{~Hz}), 3.92(\mathrm{~d}, 2 \mathrm{H}(E$ isomer $), J=2.3 \mathrm{~Hz}), 3.66(\mathrm{~d}, 2 \mathrm{H}(Z$ isomer $), J=3.5$ $\mathrm{Hz}) ;{ }^{19} \mathrm{~F}$ NMR $\left(470 \mathrm{MHz}, \mathrm{CDCl}_{3}\right) \delta-128.4(\mathrm{dm}, 1 \mathrm{~F}(\mathrm{Z}$ isomer $), J=84.1 \mathrm{~Hz}),-129.8(\mathrm{dm}$, $1 \mathrm{~F}$ ( $E$ isomer $), J=84.9 \mathrm{~Hz}) ;{ }^{13} \mathrm{C} \mathrm{NMR}\left(126 \mathrm{MHz}, \mathrm{CDCl}_{3}\right) \delta 147.7\left(\mathrm{~d}, J_{\mathrm{C}-\mathrm{F}}=46.3 \mathrm{~Hz}\right)$, $145.6\left(\mathrm{~d}, J_{\mathrm{C}-\mathrm{F}}=50.6 \mathrm{~Hz}\right), 140.8,140.6,140.5\left(\mathrm{~d}, J_{\mathrm{C}-\mathrm{F}}=0.9 \mathrm{~Hz}\right), 140.3\left(\mathrm{~d}, J_{\mathrm{C}-\mathrm{F}}=0.9 \mathrm{~Hz}\right)$, $138.9\left(\mathrm{~d}, J_{\mathrm{C}-\mathrm{F}}=2.4 \mathrm{~Hz}\right), 138.8\left(\mathrm{~d}, J_{\mathrm{C}-\mathrm{F}}=2.8 \mathrm{~Hz}\right), 135.3,135.2,133.7,128.92,128.89$, $128.81,128.77,128.7,128.62,128.60,128.58,127.49,127.46,127.4,127.18,127.16$, 127.13, 127.06, 127.0, 126.6, 126.3, $123.3\left(\mathrm{~d}, J_{\mathrm{C}-\mathrm{F}}=9.2 \mathrm{~Hz}\right), 120.9\left(\mathrm{~d}, J_{\mathrm{C}-\mathrm{F}}=1.3 \mathrm{~Hz}\right)$, $36.6\left(\mathrm{~d}, J_{\mathrm{C}-\mathrm{F}}=6.5 \mathrm{~Hz}\right), 32.8\left(\mathrm{~d}, J_{\mathrm{C}-\mathrm{F}}=4.8 \mathrm{~Hz}\right)$; HRMS-ESI calcd for $\mathrm{C}_{21} \mathrm{H}_{28} \mathrm{~F}[\mathrm{M}+\mathrm{H}]^{+}$ 289.1387; found 289.1395.
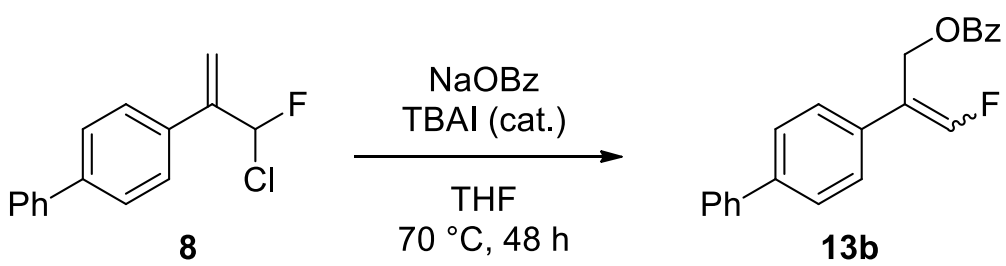

2-([1,1'-Biphenyl]-4-yl)-3-fluoroallyl benzoate (13b). An argon-flushed vial was charged with sodium benzoate $(58 \mathrm{mg}, 0.405 \mathrm{mmol})$, tetra- $n$-butylammonium iodide $(7.5$ $\mathrm{mg}, 20.3 \mu \mathrm{mol})$ and THF $(1.0 \mathrm{~mL})$. A solution of $1(50 \mathrm{mg}, 0.203 \mathrm{mmol})$ in THF $(1 \mathrm{ml})$ was next added. The resulting mixture was stirred at $70{ }^{\circ} \mathrm{C}$ for 48 hours. Water was added and the mixture was extracted with $\mathrm{Et}_{2} \mathrm{O}(3 \mathrm{x})$. The organic layers were combined, washed with brine $(1 \mathrm{x})$, dried over $\mathrm{MgSO}_{4}$ and concentrated in vacuo. The desired product $(47 \mathrm{mg}, 70 \%, E / Z=31: 69)$ was isolated as a colorless oil using 10\% $\mathrm{Et}_{2} \mathrm{O} /$ hexanes. IR $(\mathrm{ATR}, \mathrm{ZnSe}) v=3030,2922,1714,1653,1487,1450,1263$, 1094, 958, 
833, 763, $708 \mathrm{~cm}^{-1} ;{ }^{1} \mathrm{H}$ NMR (500 MHz, $\left.\mathrm{CDCl}_{3}\right) \delta 8.01-7.97(\mathrm{~m}, 2 \mathrm{H}), 7.67-7.51(\mathrm{~m}, 6 \mathrm{H})$, 7.47-7.33 (m, 6H), 7.07 (d, $1 \mathrm{H}$ ( $E$ isomer), $J=81.3 \mathrm{~Hz}$ ), 7.06 (d, $1 \mathrm{H}$ ( $Z$ isomer), $J=82.7$ $\mathrm{Hz}), 5.35$ (d, $2 \mathrm{H}(Z$ isomer), $J=2.8 \mathrm{~Hz}), 5.11(\mathrm{~d}, 2 \mathrm{H}(E$ isomer $), J=3.9 \mathrm{~Hz}) ;{ }^{19} \mathrm{~F}$ NMR $\left(470 \mathrm{MHz}, \mathrm{CDCl}_{3}\right) \delta-122.0$ (dt, $1 \mathrm{~F}$ ( $E$ isomer), $\left.J=81.4,3.7 \mathrm{~Hz}\right),-124.6(\mathrm{~d}, 1 \mathrm{~F}(Z$ isomer), $J=82.7 \mathrm{~Hz}) ;{ }^{13} \mathrm{C}$ NMR $\left(126 \mathrm{MHz}, \mathrm{CDCl}_{3}\right) \delta 166.54,166.50,149.7\left(\mathrm{~d}, J_{\mathrm{C}-\mathrm{F}}=\right.$ $273 \mathrm{~Hz}), 149.2\left(\mathrm{~d}, J_{\mathrm{C}-\mathrm{F}}=269 \mathrm{~Hz}\right), 141.0\left(\mathrm{~d}, J_{\mathrm{C}-\mathrm{F}}=0.8 \mathrm{~Hz}\right), 140.9\left(\mathrm{~d}, J_{\mathrm{C}-\mathrm{F}}=0.9 \mathrm{~Hz}\right)$, 140.6, 140.5, 133.3, 133.2, $133.1\left(\mathrm{~d}, J_{\mathrm{C}-\mathrm{F}}=7.2 \mathrm{~Hz}\right), 131.8\left(\mathrm{~d}, J_{\mathrm{C}-\mathrm{F}}=1.4 \mathrm{~Hz}\right), 130.03$, $130.00,129.81,129.80,128.98,128.96,128.6,128.53,128.51,127.65,127.59,127.3$, $127.2,127.13,127.11,120.1\left(\mathrm{~d}, J_{\mathrm{C}-\mathrm{F}}=6.6 \mathrm{~Hz}\right), 117.8\left(\mathrm{~d}, J_{\mathrm{C}-\mathrm{F}}=2.9 \mathrm{~Hz}\right), 63.6\left(\mathrm{~d}, J_{\mathrm{C}-\mathrm{F}}=\right.$ $12.3 \mathrm{~Hz}), 58.8\left(\mathrm{~d}, J_{\mathrm{C}-\mathrm{F}}=7.8 \mathrm{~Hz}\right)$; HRMS-ESI calcd for $\mathrm{C}_{22} \mathrm{H}_{18} \mathrm{FO}_{2}[\mathrm{M}+\mathrm{H}]^{+} 333.1285$; found 333.1297 .

\section{Synthetic transformations of monofluoroalkenes}

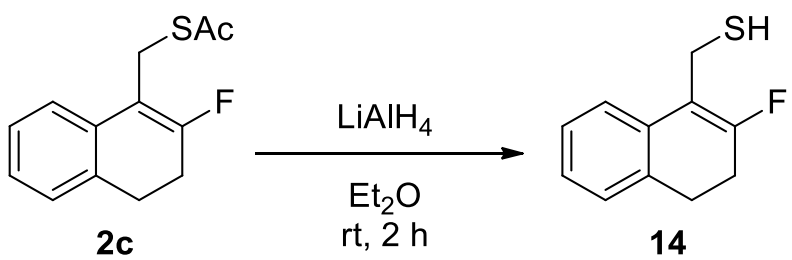

(2-Fluoro-3,4-dihydronaphthalen-1-yl)methanethiol (14). A solution of $2 \mathrm{c}(50 \mathrm{mg}$, $0.212 \mathrm{mmol})$ in $\mathrm{Et}_{2} \mathrm{O}(1.1 \mathrm{~mL})$ was added dropwise at ambient temperature to a suspension of $\mathrm{LiAlH}_{4}(16 \mathrm{mg}, 0.425 \mathrm{mmol})$ in $\mathrm{Et}_{2} \mathrm{O}(1 \mathrm{~mL})$. The resulting mixture was stirred at ambient temperature for 2 hours. A saturated solution of $\mathrm{NH}_{4} \mathrm{Cl}$ was added slowly, followed by $\mathrm{HCl}(3 \mathrm{M})$ to dissolve the solids. The aqueous mixture was extracted with $\mathrm{Et}_{2} \mathrm{O}(3 \mathrm{x})$. The organic layers were combined, washed with $\mathrm{Na}_{2} \mathrm{CO}_{3}$ sat. (1x) water (1x), brine (1x), dried over $\mathrm{Na}_{2} \mathrm{SO}_{4}$ and concentrated in vacuo. The desired product (39 $\mathrm{mg}, 94 \%$ ) was directly obtained as a pale yellow oil. IR (ATR, ZnSe) $v=3018,2942$, 2834, 1675, 1487, 1436, 1334, 1149, 1002, $754 \mathrm{~cm}^{-1} ;{ }^{1} \mathrm{H}$ NMR (500 MHz, $\left.\mathrm{CDCl}_{3}\right) \delta$ 7.26-7.22 (m, 2H), 7.16-7.13 (m, 2H), 3.65 (d, 2H, $J=7.0 \mathrm{~Hz}$ ), 2.97 (td, $2 \mathrm{H}, J=7.7,2.5$ $\mathrm{Hz}), 2.57(\mathrm{~m}, 2 \mathrm{H}), 1.78(\mathrm{t}, 1 \mathrm{H}, J=7.4 \mathrm{~Hz}) ;{ }^{19} \mathrm{~F} \mathrm{NMR}\left(470 \mathrm{MHz}, \mathrm{CDCl}_{3}\right) \delta-101.4(\mathrm{~s}, 1 \mathrm{~F})$; ${ }^{13} \mathrm{C} \mathrm{NMR}\left(126 \mathrm{MHz}, \mathrm{CDCl}_{3}\right) \delta 159.2\left(\mathrm{~d}, J_{\mathrm{C}-\mathrm{F}}=270 \mathrm{~Hz}\right), 133.2,132.4\left(\mathrm{~d}, J_{\mathrm{C}-\mathrm{F}}=5.6 \mathrm{~Hz}\right)$, 
127.8, 127.0, $126.6\left(\mathrm{~d}, J_{\mathrm{C}-\mathrm{F}}=2.2 \mathrm{~Hz}\right), 122.9\left(\mathrm{~d}, J_{\mathrm{C}-\mathrm{F}}=6.8 \mathrm{~Hz}\right), 114.4\left(\mathrm{~d}, J_{\mathrm{C}-\mathrm{F}}=12.5 \mathrm{~Hz}\right)$, $28.5\left(\mathrm{~d}, J_{\mathrm{C}-\mathrm{F}}=7.3 \mathrm{~Hz}\right), 24.9\left(\mathrm{~d}, J_{\mathrm{C}-\mathrm{F}}=24.0 \mathrm{~Hz}\right), 17.4\left(\mathrm{~d}, J_{\mathrm{C}-\mathrm{F}}=7.0 \mathrm{~Hz}\right) ;$ HRMS-ESI calcd for $\mathrm{C}_{11} \mathrm{H}_{10} \mathrm{~F}[\mathrm{M}+\mathrm{H}]^{+}-\left[\mathrm{H}_{2} \mathrm{~S}\right]$ 161.0761; found 161.0759.

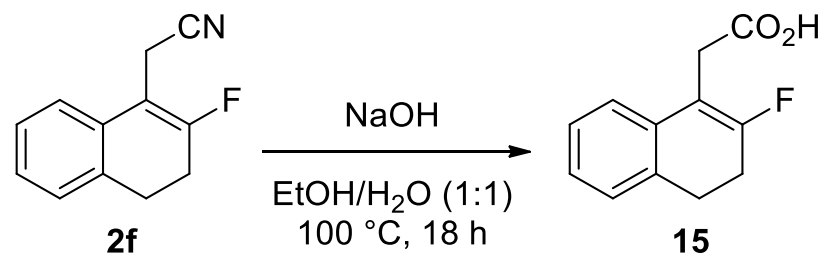

2-(2-Fluoro-3,4-dihydronaphthalen-1-yl)acetic acid (15). To a vial charged with a solution of $\mathrm{NaOH}(46 \mathrm{mg}, 1.15 \mathrm{mmol})$ in water $(1.15 \mathrm{~mL})$ was added a solution of $2 \mathbf{f}$ (43 $\mathrm{mg}, 0.230 \mathrm{mmol})$ in EtOH $(1.15 \mathrm{~mL})$. The resulting mixture was stirred at $100{ }^{\circ} \mathrm{C}$ for 18 hours. The mixture was cooled and diluted with $\mathrm{NaOH}(0.1 \mathrm{M})$. The aqueous mixture was washed with $\mathrm{CH}_{2} \mathrm{Cl}_{2}(2 \mathrm{x}) . \mathrm{HCl}(3 \mathrm{M})$ was added until $\mathrm{pH}=1$. The aqueous mixture was extracted with $\mathrm{CH}_{2} \mathrm{Cl}_{2}(3 \mathrm{x})$. The organic layers were combined, dried over $\mathrm{Na}_{2} \mathrm{SO}_{4}$ and concentrated in vacuo. The desired product $(35 \mathrm{mg}, 74 \%)$ was isolated as a graygreen solid by flash chromatography using $3 \% \mathrm{MeOH} / \mathrm{CH}_{2} \mathrm{Cl}_{2}$. mp $94-97{ }^{\circ} \mathrm{C}$; IR (ATR, $\mathrm{ZnSe}) v=3019,2922,2613,1705,1686,1414,1234,1000,922,767 \mathrm{~cm}^{-1} ;{ }^{1} \mathrm{H}$ NMR (500 MHz, DMSO-d $\left.{ }_{6}\right) \delta 12.42$ (bs, 1H), 7.20-7.15 (m, 2H), 7.13-7.10 (m, 2H), $3.43(\mathrm{~s}, 2 \mathrm{H})$, $2.95(\mathrm{td}, 2 \mathrm{H}, J=8.2,1.8 \mathrm{~Hz}), 2.57(\mathrm{~m}, 2 \mathrm{H}) ;{ }^{19} \mathrm{~F}$ NMR $(470 \mathrm{MHz}$, DMSO-d 6$) \delta-101.5(\mathrm{~s}$, 1F); ${ }^{13} \mathrm{C}$ NMR (126 MHz, DMSO-d 6$) \delta 171.9\left(\mathrm{~d}, J_{\mathrm{C}-\mathrm{F}}=2.6 \mathrm{~Hz}\right), 159.9\left(\mathrm{~d}, J_{\mathrm{C}-\mathrm{F}}=267 \mathrm{~Hz}\right)$, $133.5\left(\mathrm{~d}, J_{\mathrm{C}-\mathrm{F}}=6.2 \mathrm{~Hz}\right), 132.5,127.4,126.6,126.2\left(\mathrm{~d}, J_{\mathrm{C}-\mathrm{F}}=2.1 \mathrm{~Hz}\right), 122.8\left(\mathrm{~d}, J_{\mathrm{C}-\mathrm{F}}=6.6\right.$ $\mathrm{Hz}), 108.7\left(\mathrm{~d}, J_{\mathrm{C}-\mathrm{F}}=13.6 \mathrm{~Hz}\right), 29.4\left(\mathrm{~d}, J_{\mathrm{C}-\mathrm{F}}=5.2 \mathrm{~Hz}\right), 27.8\left(\mathrm{~d}, J_{\mathrm{C}-\mathrm{F}}=7.6 \mathrm{~Hz}\right), 24.1\left(\mathrm{~d}, J_{\mathrm{C}-\mathrm{F}}\right.$ $=24.0 \mathrm{~Hz}$ ); HRMS-ESI calcd for $\mathrm{C}_{12} \mathrm{H}_{12} \mathrm{FO}_{2}[\mathrm{M}+\mathrm{H}]^{+}$207.0816; found 207.0832.

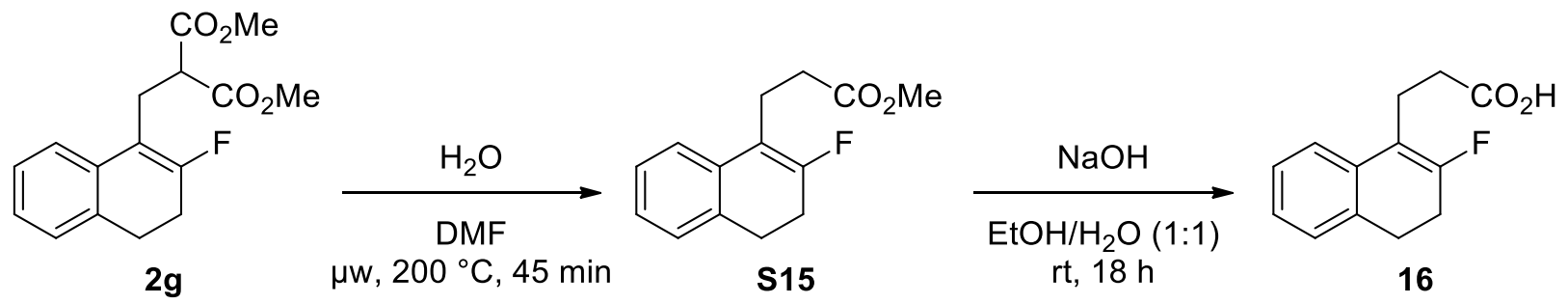


Methyl 3-(2-fluoro-3,4-dihydronaphthalen-1-yl)propanoate (S15). $\mathrm{H}_{2} \mathrm{O} \quad(8.7 \mathrm{mg}$, $0.484 \mathrm{mmol}$ ) was weighted in a microwave vial, and a solution of $\mathbf{2 g}$ (59 $\mathrm{mg}, 0.202$ mmol) in DMF (2 mL) was next added. The mixture was stirred at $200{ }^{\circ} \mathrm{C}$ for 45 minutes under microwave irradiation, and then brought back to ambient temperature. $\mathrm{Et}_{2} \mathrm{O}$ and water were added, and the layers were separated. The ethereal layer was washed with water $(1 \mathrm{x})$, brine $(1 \mathrm{x})$, dried over $\mathrm{Na}_{2} \mathrm{SO}_{4}$ and concentrated in vacuo. The desired product (40 mg, 84\%) was isolated as a colorless oil by flash chromatography using 15\% $\mathrm{Et}_{2} \mathrm{O} /$ hexanes. IR (ATR, ZnSe) $v=2950,2848,1734,1681,1436,1366,1149,1036,908$, $729 \mathrm{~cm}^{-1} ;{ }^{1} \mathrm{H}$ NMR (500 MHz, $\left.\mathrm{CDCl}_{3}\right) \delta$ 7.22-7.18 (m, 2H), 7.12-7.09 (m, 2H), $3.68(\mathrm{~s}$, $3 \mathrm{H}), 2.94(\mathrm{td}, 2 \mathrm{H}, J=8.2,2.5 \mathrm{~Hz}), 2.85(\mathrm{t}, 2 \mathrm{H}, J=7.8 \mathrm{~Hz}), 2.55-2.49(\mathrm{~m}, 4 \mathrm{H}) ;{ }^{19} \mathrm{~F} \mathrm{NMR}$ $\left(470 \mathrm{MHz}, \mathrm{CDCl}_{3}\right) \delta-102.8(\mathrm{~s}, 1 \mathrm{~F}) ;{ }^{13} \mathrm{C} \mathrm{NMR}\left(126 \mathrm{MHz}, \mathrm{CDCl}_{3}\right) \delta 173.6,159.4\left(\mathrm{~d}, J_{\mathrm{C}-\mathrm{F}}\right.$ $=267 \mathrm{~Hz}), 133.7\left(\mathrm{~d}, J_{\mathrm{C}-\mathrm{F}}=6.9 \mathrm{~Hz}\right), 133.3,127.7,126.9,126.2\left(\mathrm{~d}, J_{\mathrm{C}-\mathrm{F}}=2.0 \mathrm{~Hz}\right), 122.6$ $\left(\mathrm{d}, J_{\mathrm{C}-\mathrm{F}}=6.7 \mathrm{~Hz}\right), 112.9\left(\mathrm{~d}, J_{\mathrm{C}-\mathrm{F}}=13.7 \mathrm{~Hz}\right), 51.8,33.1\left(\mathrm{~d}, J_{\mathrm{C}-\mathrm{F}}=2.1 \mathrm{~Hz}\right), 28.9\left(\mathrm{~d}, J_{\mathrm{C}-\mathrm{F}}=\right.$ $7.3 \mathrm{~Hz}), 24.9\left(\mathrm{~d}, J_{\mathrm{C}-\mathrm{F}}=24.8 \mathrm{~Hz}\right), 19.2\left(\mathrm{~d}, J_{\mathrm{C}-\mathrm{F}}=5.3 \mathrm{~Hz}\right)$; HRMS-ESI calcd for $\mathrm{C}_{14} \mathrm{H}_{16} \mathrm{FO}_{2}$ $[\mathrm{M}+\mathrm{H}]^{+}$235.1129; found 235.1129.

3-(2-Fluoro-3,4-dihydronaphthalen-1-yl)propanoic acid (16). To a solution of S15 (39 $\mathrm{mg}, 0.167 \mathrm{mmol})$ in $\mathrm{EtOH}(0.7 \mathrm{~mL})$ was added aqueous $\mathrm{NaOH}(0.5 \mathrm{M}, 0.7 \mathrm{~mL}, 0.333$ $\mathrm{mmol})$. The resulting mixture was stirred at ambient temperature for 18 hours. $\mathrm{HCl}(3 \mathrm{M})$ was added, and the aqueous mixture was extracted with EtOAc (3x). The organic layers were combined, washed with brine $(1 \mathrm{x})$, dried over $\mathrm{Na}_{2} \mathrm{SO}_{4}$ and concentrated in vacuo. The desired product (33 mg, 89\%) was isolated as an amber oil by flash chromatography using $2 \% \mathrm{MeOH} / \mathrm{CH}_{2} \mathrm{Cl}_{2}$. IR (ATR, ZnSe) $v=2939,2836,1702,1680,1410,1296$, 1225, 1148, 924, $729 \mathrm{~cm}^{-1} ;{ }^{1} \mathrm{H}$ NMR $\left(500 \mathrm{MHz}, \mathrm{CDCl}_{3}\right) \delta 11.39$ (bs, 1H), 7.23-7.18 (m, 2H), 7.13-7.10 (m, 2H), $2.95(\mathrm{td}, 2 \mathrm{H}, J=8.2,2.2 \mathrm{~Hz}), 2.87$ (t, 2H, $J=7.7 \mathrm{~Hz}), 2.58-2.52$ $(\mathrm{m}, 2 \mathrm{H}) ;{ }^{19} \mathrm{~F}$ NMR $\left(470 \mathrm{MHz}, \mathrm{CDCl}_{3}\right) \delta-102.6(\mathrm{~s}, 1 \mathrm{~F}) ;{ }^{13} \mathrm{C} \mathrm{NMR}\left(126 \mathrm{MHz}, \mathrm{CDCl}_{3}\right) \delta$ $179.2,159.5\left(\mathrm{~d}, J_{\mathrm{C}-\mathrm{F}}=267 \mathrm{~Hz}\right), 133.6\left(\mathrm{~d}, J_{\mathrm{C}-\mathrm{F}}=6.5 \mathrm{~Hz}\right), 133.3,127.8,127.0,126.3\left(\mathrm{~d}, J_{\mathrm{C}-}\right.$ $\mathrm{F}=2.1 \mathrm{~Hz}), 122.5\left(\mathrm{~d}, J_{\mathrm{C}-\mathrm{F}}=6.7 \mathrm{~Hz}\right), 112.6\left(\mathrm{~d}, J_{\mathrm{C}-\mathrm{F}}=13.6 \mathrm{~Hz}\right), 32.9\left(\mathrm{~d}, J_{\mathrm{C}-\mathrm{F}}=2.1 \mathrm{~Hz}\right)$, $28.8\left(\mathrm{~d}, J_{\mathrm{C}-\mathrm{F}}=7.3 \mathrm{~Hz}\right), 24.9\left(\mathrm{~d}, J_{\mathrm{C}-\mathrm{F}}=24.7 \mathrm{~Hz}\right), 18.9\left(\mathrm{~d}, J_{\mathrm{C}-\mathrm{F}}=5.4 \mathrm{~Hz}\right)$; HRMS-ESI calcd for $\mathrm{C}_{13} \mathrm{H}_{13} \mathrm{FNaO}_{2}$ 243.0792; found 243.0793. 


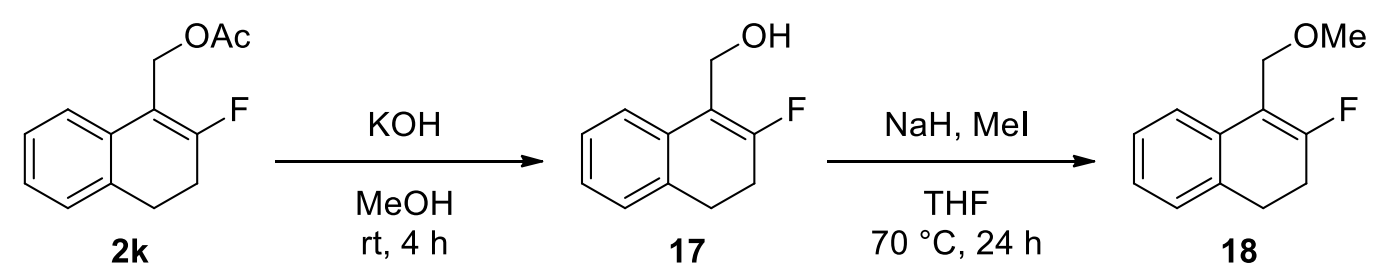

(2-Fluoro-3,4-dihydronaphthalen-1-yl)methanol (17). KOH (33 mg, $0.584 \mathrm{mmol}$ ) was added to a solution of $2 \mathbf{k}$ (46 mg, $0.195 \mathrm{mmol})$ in $\mathrm{MeOH}(1 \mathrm{~mL})$ and the resulting mixture was stirred at ambient temperature for 4 hours. Water was added, and the mixture was extracted with $\mathrm{CH}_{2} \mathrm{Cl}_{2}$ (3x). The organic layers were combined, washed with water (1x), dried over $\mathrm{Na}_{2} \mathrm{SO}_{4}$ and concentrated in vacuo. The desired product (36 mg, 93\%) was isolated as a colorless oil by flash chromatography using $\mathrm{CH}_{2} \mathrm{Cl}_{2}$. IR (ATR, $\mathrm{ZnSe}$ ) $v$ $=3324,2942,2890,1679,1488,1365,1226,1151,997,759 \mathrm{~cm}^{-1} ;{ }^{1} \mathrm{H}$ NMR $(500 \mathrm{MHz}$, $\left.\mathrm{CDCl}_{3}\right) \delta 7.43(\mathrm{~d}, 1 \mathrm{H}, J=7.7 \mathrm{~Hz}), 7.23(\mathrm{~m}, 1 \mathrm{H}), 7.15-7.13(\mathrm{~m}, 2 \mathrm{H}), 4.65(\mathrm{~s}, 2 \mathrm{H}), 2.98$ $(\mathrm{td}, 2 \mathrm{H}, J=8.2,2.4 \mathrm{~Hz}), 2.58(\mathrm{~m}, 2 \mathrm{H}), 1.50(\mathrm{bs}, 1 \mathrm{H}) ;{ }^{19} \mathrm{~F} \mathrm{NMR}\left(470 \mathrm{MHz}, \mathrm{CDCl}_{3}\right)$ $\delta$-102.5 (s, 1F); ${ }^{13} \mathrm{C}$ NMR $\left(126 \mathrm{MHz}, \mathrm{CDCl}_{3}\right) \delta 160.7\left(\mathrm{~d}, J_{\mathrm{C}-\mathrm{F}}=271 \mathrm{~Hz}\right), 133.0\left(\mathrm{~d}, J_{\mathrm{C}-\mathrm{F}}=\right.$ $5.8 \mathrm{~Hz}), 132.9,127.7,127.0,126.6\left(\mathrm{~d}, J_{\mathrm{C}-\mathrm{F}}=2.0 \mathrm{~Hz}\right), 123.5\left(\mathrm{~d}, J_{\mathrm{C}-\mathrm{F}}=6.6 \mathrm{~Hz}\right), 114.2(\mathrm{~d}$, $\left.J_{\mathrm{C}-\mathrm{F}}=11.6 \mathrm{~Hz}\right), 54.8\left(\mathrm{~d}, J_{\mathrm{C}-\mathrm{F}}=7.4 \mathrm{~Hz}\right), 28.5\left(\mathrm{~d}, J_{\mathrm{C}-\mathrm{F}}=7.2 \mathrm{~Hz}\right), 24.9\left(\mathrm{~d}, J_{\mathrm{C}-\mathrm{F}}=24.4 \mathrm{~Hz}\right)$; HRMS-ESI calcd for $\mathrm{C}_{11} \mathrm{H}_{10} \mathrm{~F}[\mathrm{M}+\mathrm{H}]^{+}-\left[\mathrm{H}_{2} \mathrm{O}\right]$ 161.0761; found 161.0775.

3-Fluoro-4-(methoxymethyl)-1,2-dihydronaphthalene (18). An argon-flushed vial was charged with $\mathrm{NaH}(60 \%$ in mineral oil, $16 \mathrm{mg}, 0.416 \mathrm{mmol})$ and THF $(2 \mathrm{~mL})$. A solution of $17(37 \mathrm{mg}, 0.208 \mathrm{mmol})$ in THF ( $2 \mathrm{~mL}$ ) was added slowly, and the resulting mixture was stirred at ambient temperature for 10 minutes. MeI ( $26 \mu \mathrm{L}, 0.416 \mathrm{mmol})$ was added, after which the solution was stirred at $70{ }^{\circ} \mathrm{C}$ for 24 hours. Water was added and the mixture was extracted with $\mathrm{Et}_{2} \mathrm{O}(3 \mathrm{x})$. The organic layers were combined, washed with brine (1x), dried over $\mathrm{Na}_{2} \mathrm{SO}_{4}$ and concentrated in vacuo. The desired product (36 mg, 91\%) was isolated as a colorless oil by flash chromatography using $5 \% \mathrm{Et}_{2} \mathrm{O} /$ hexanes. IR (ATR, ZnSe) $v=2926,2891,1680,1488,1370,1151,1092,1016,907,758,723 \mathrm{~cm}^{-1}$; ${ }^{1} \mathrm{H}$ NMR $\left(500 \mathrm{MHz}, \mathrm{CDCl}_{3}\right) \delta 7.38(\mathrm{~d}, 1 \mathrm{H}, J=7.7 \mathrm{~Hz}), 7.21(\mathrm{~m}, 1 \mathrm{H}), 7.14-7.11(\mathrm{~m}, 2 \mathrm{H})$, $4.43(\mathrm{~d}, J=2.1 \mathrm{~Hz}), 3.38$ (s, 3H), 2.99 (td, $2 \mathrm{H}, J=8.2,2.3 \mathrm{~Hz}), 2.60$ (m, 2H); ${ }^{19} \mathrm{~F}$ NMR $\left(470 \mathrm{MHz}, \mathrm{CDCl}_{3}\right) \delta-101.6(\mathrm{~s}, 1 \mathrm{~F}) ;{ }^{13} \mathrm{C} \mathrm{NMR}\left(126 \mathrm{MHz}, \mathrm{CDCl}_{3}\right) \delta 161.5\left(\mathrm{~d}, J_{\mathrm{C}-\mathrm{F}}=271\right.$ $\mathrm{Hz}), 133.4\left(\mathrm{~d}, J_{\mathrm{C}-\mathrm{F}}=5.8 \mathrm{~Hz}\right), 132.7,127.4,127.0,126.4\left(\mathrm{~d}, J_{\mathrm{C}-\mathrm{F}}=2.0 \mathrm{~Hz}\right), 123.9\left(\mathrm{~d}, J_{\mathrm{C}-\mathrm{F}}\right.$ 
$=6.6 \mathrm{~Hz}), 111.3\left(\mathrm{~d}, J_{\mathrm{C}-\mathrm{F}}=11.4 \mathrm{~Hz}\right), 63.8\left(\mathrm{~d}, J_{\mathrm{C}-\mathrm{F}}=6.2 \mathrm{~Hz}\right), 57.7,28.6\left(\mathrm{~d}, J_{\mathrm{C}-\mathrm{F}}=7.2 \mathrm{~Hz}\right)$, $25.0\left(\mathrm{~d}, J_{\mathrm{C}-\mathrm{F}}=24.6 \mathrm{~Hz}\right)$; HRMS-ESI calcd for $[\mathrm{M}+\mathrm{H}]^{+}-[\mathrm{MeOH}]$ 161.0761; found 161.0765 .

\section{References}

${ }^{1}$ Shibatomi, K.; Yamamoto, H. Angew. Chem. Int. Ed. 2008, 47, 5796-5798.

${ }^{2}$ Liu, J.; Chan, J.; Bryant, C. M.; Duspara, P. A.; Lee, E. E.; Powell, D.; Yang, H.; Liu, Z.; Walpole, C.; Roberts, E.; Batey, R. A. Tetrahedron Lett. 2012, 53, 2971-2975.

${ }^{3}$ Bergeron, M.; Johnson, T.; Paquin, J.-F. Angew. Chem. Int. Ed. 2011, 50, 11112-11116.

${ }^{4}$ Stavber, S.; Šket, B.; Zajc, B.; Zupan, M. Tetrahedron 1989, 45, 6003-6010.

${ }^{5}$ Stavber, S.; Jereb, M.; Zupan, M. Synthesis 2002, 17, 2609-2615.

${ }^{6}$ Bélanger, É.; Cantin, K.; Messe, O.; Tremblay, M.; Paquin, J.-F. J. Am. Chem. Soc. 2007, 129, 1034-1035.

${ }^{7}$ Krane Thvedt, T. H.; Kaasa, K.; Sundby, E.; Charnock, C.; Helge Hoff, B. Eur. J. Med. Chem. 2013, 68, 482-496.

${ }^{8}$ Shao, T.; Fang, X.; Yang, X. Synlett 2015, 26, 1835-1840.

${ }^{9}$ Pigeon, X.; Bergeron, M.; Barabé, F.; Dubé, P.; Frost, H. N.; Paquin, J.-F. Angew. Chem. Int. Ed. 2010, 49, 1123-1127.

${ }^{10}$ Bergeron M.; Guyader, D.; Paquin, J.-F. Org. Lett. 2012, 14, 5888-5891. 
6. ${ }^{1} \mathrm{H},{ }^{19} \mathrm{~F}$ and ${ }^{13} \mathrm{C}$ spectra

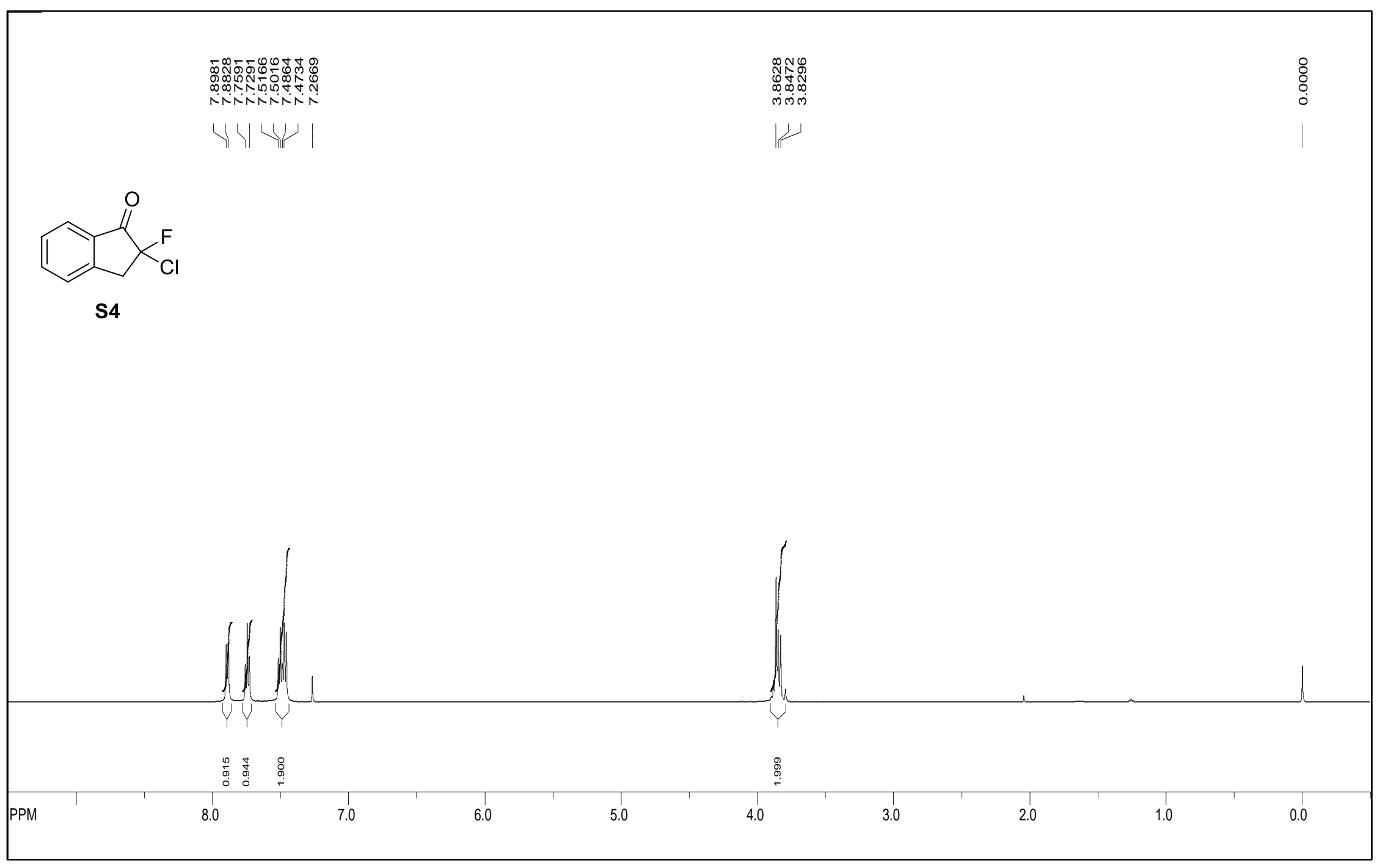


SI-34

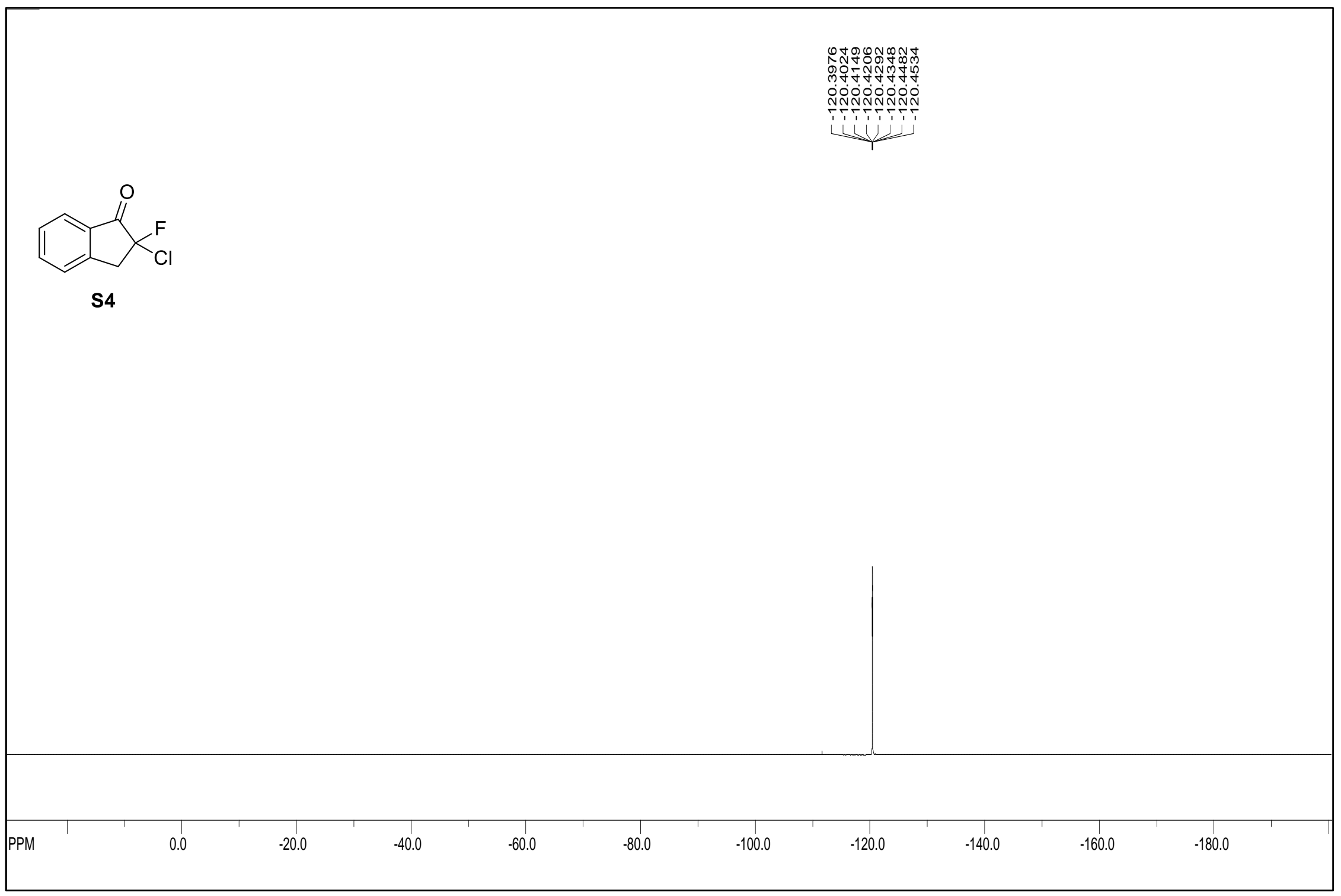




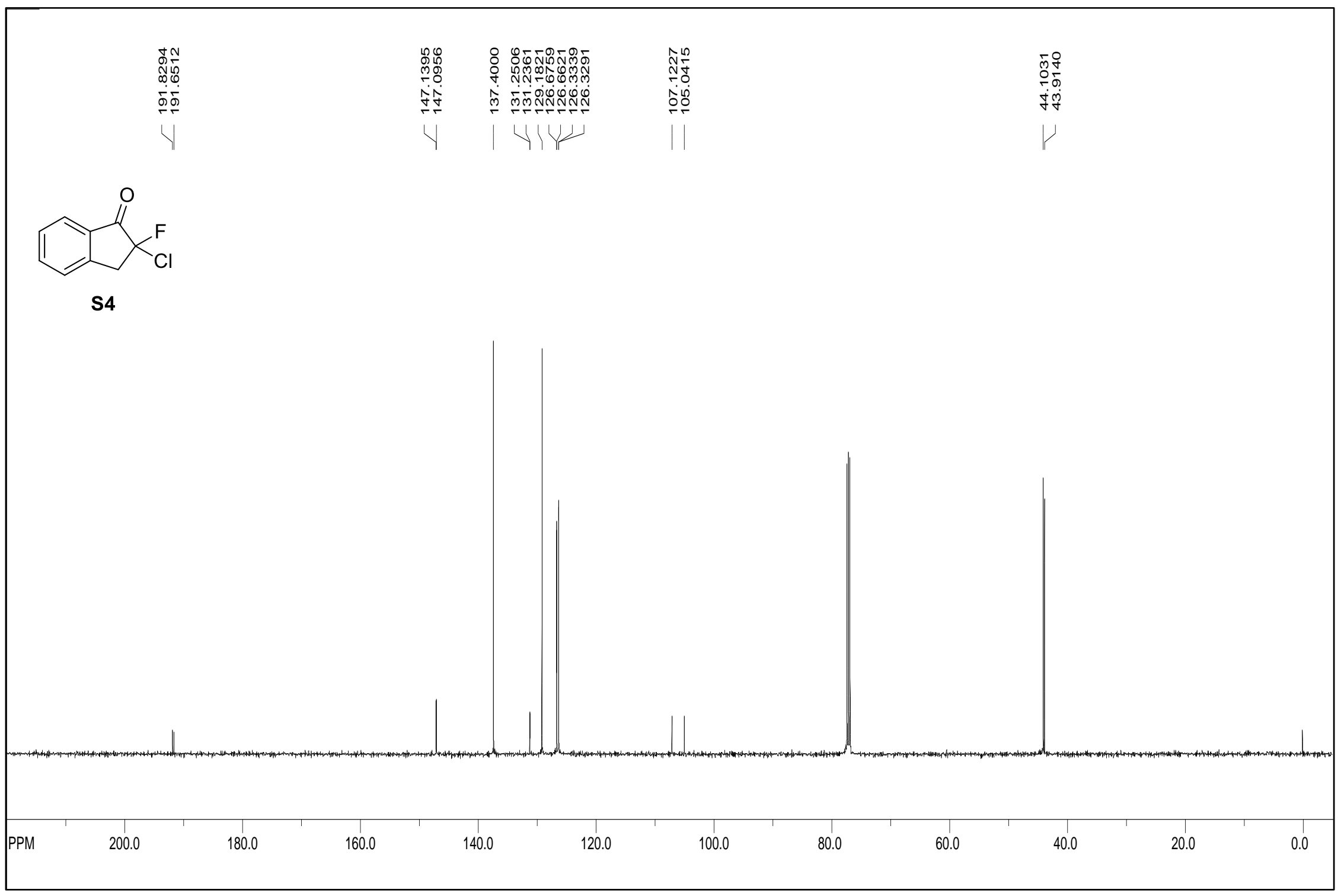




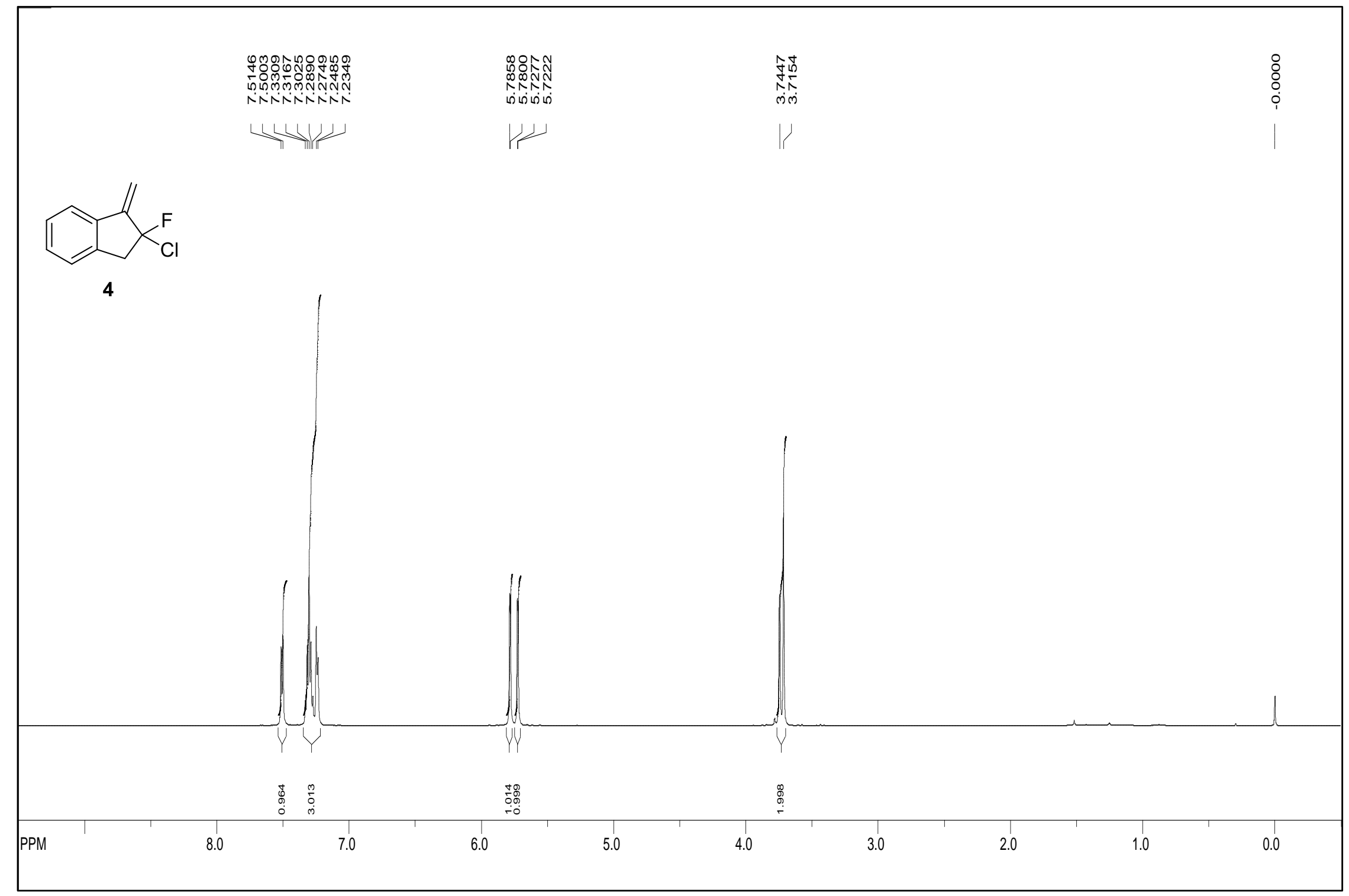




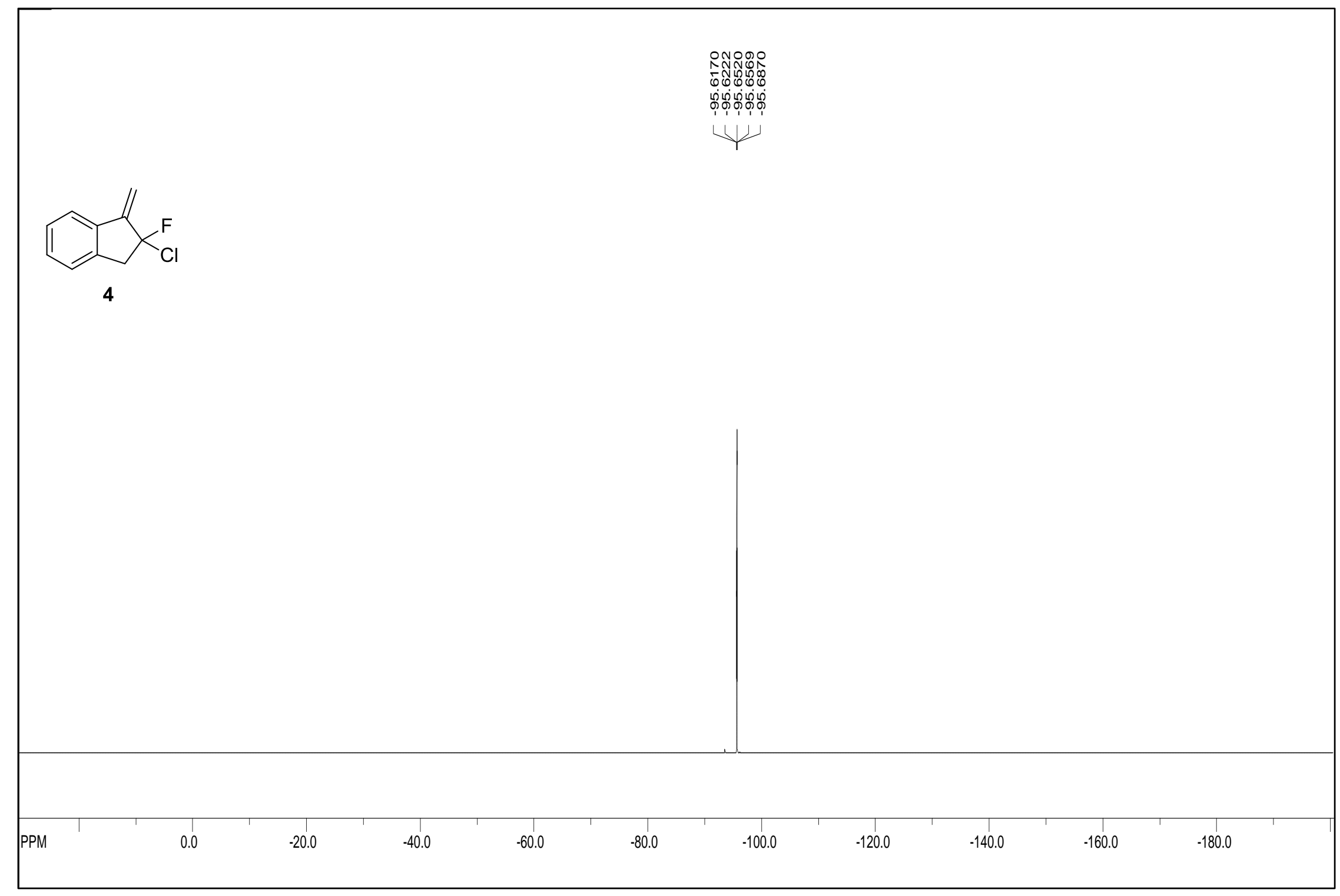




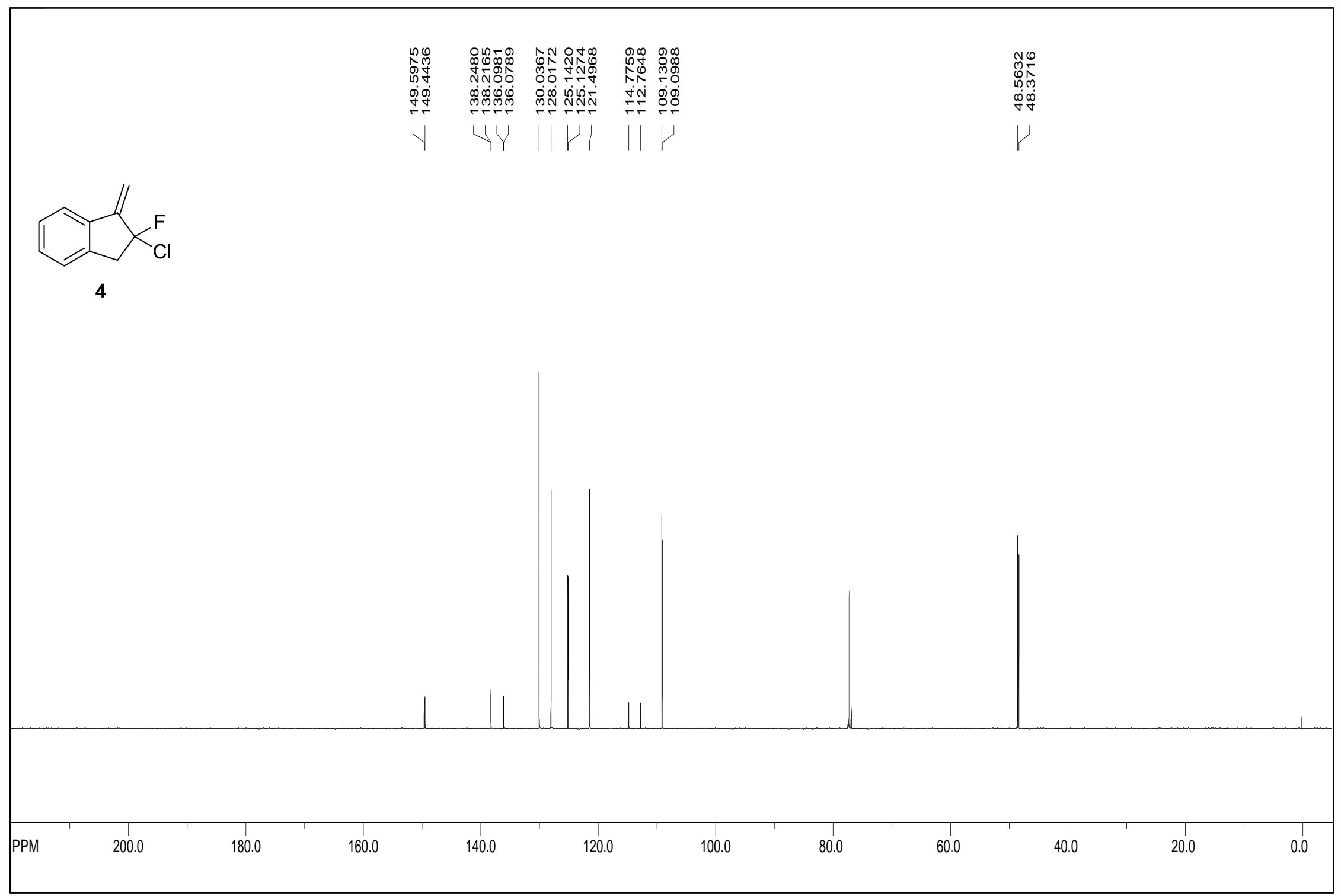




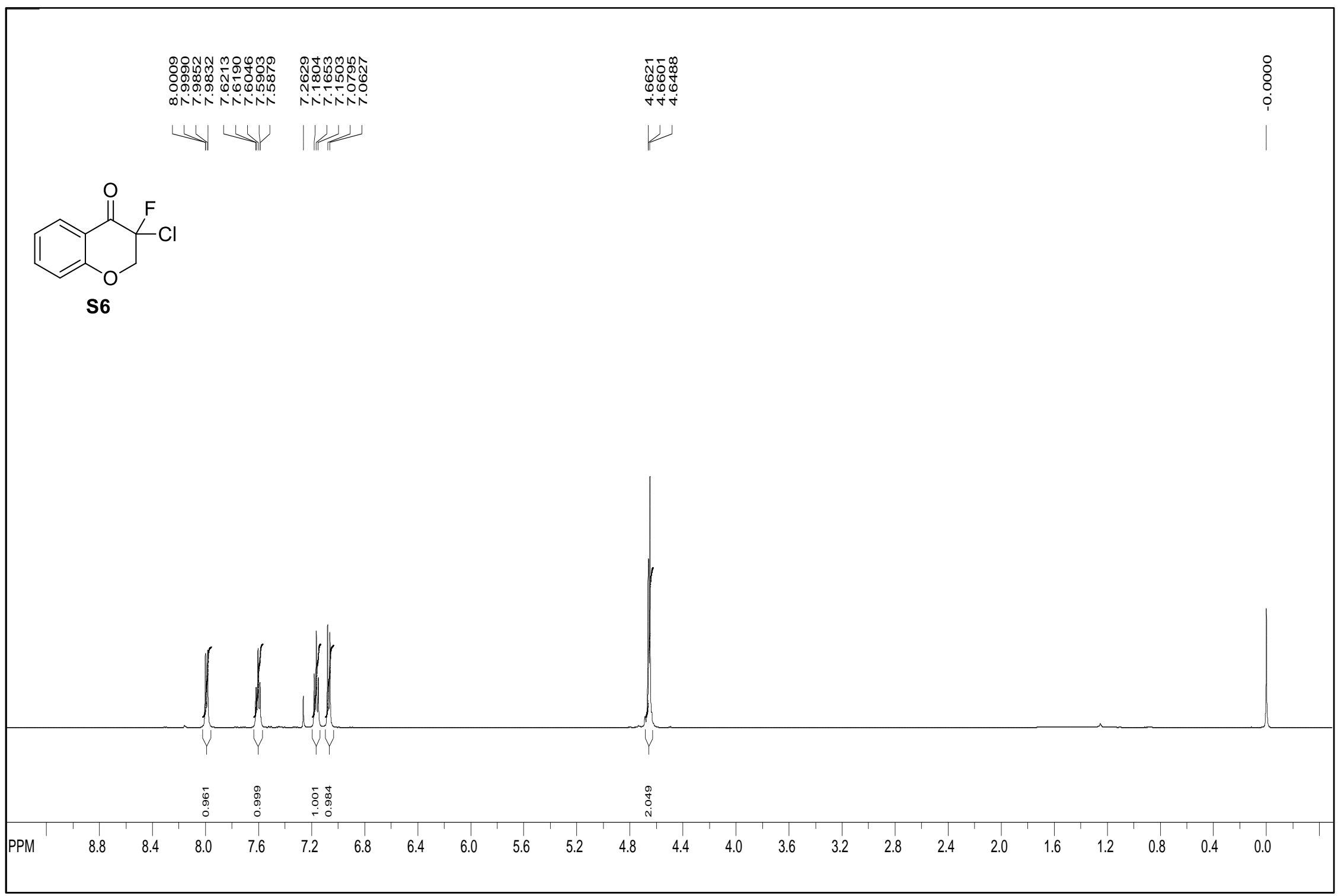




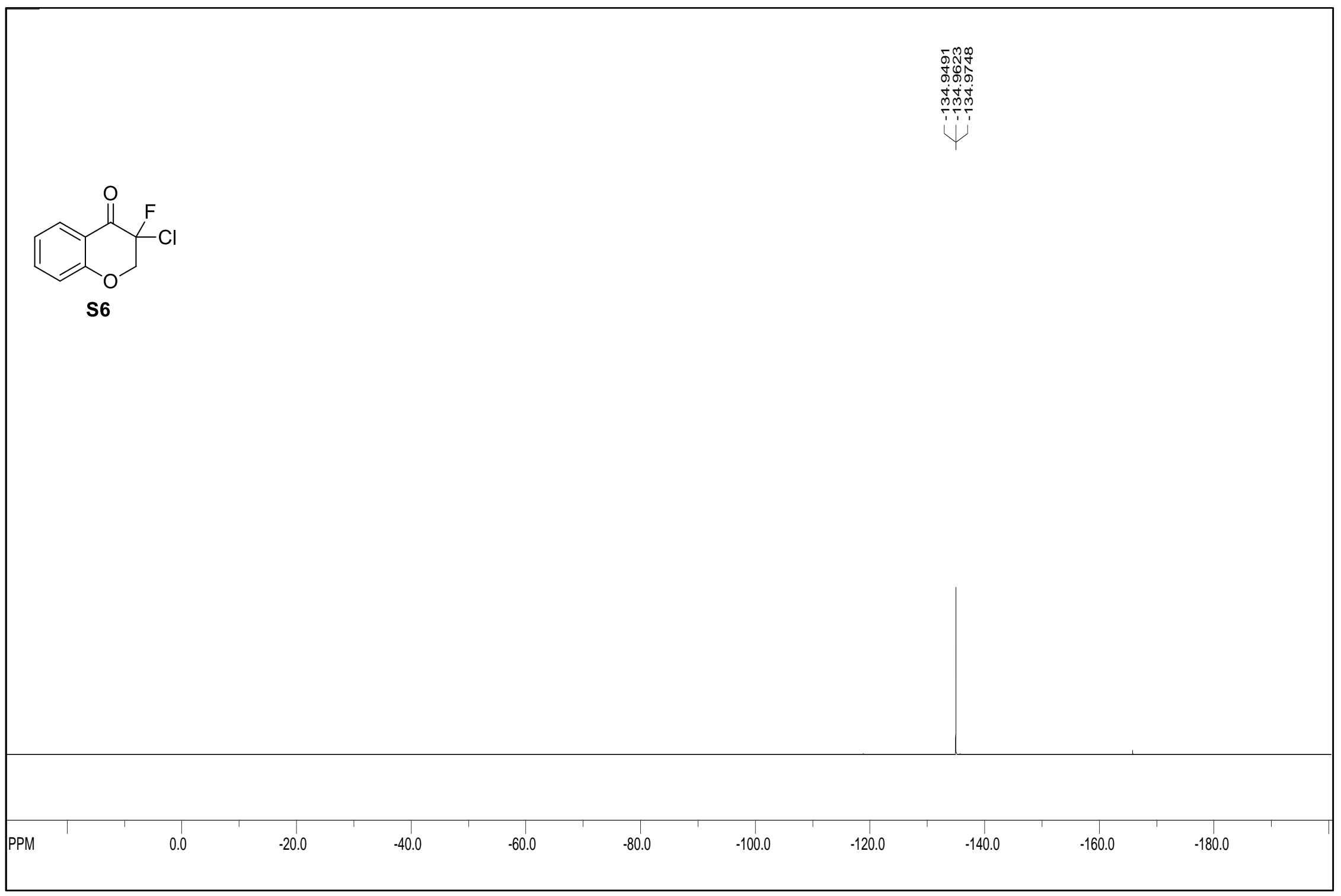




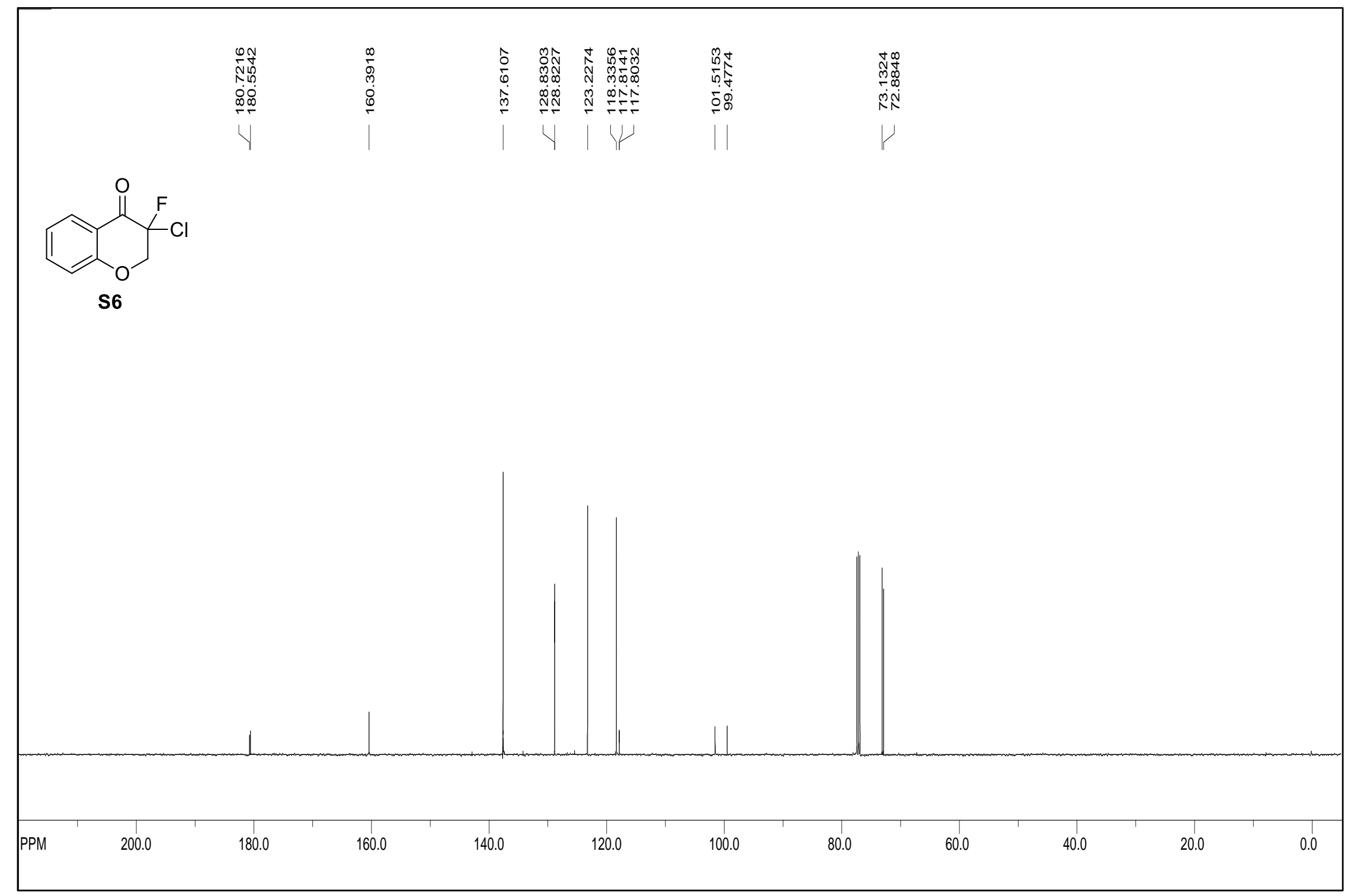




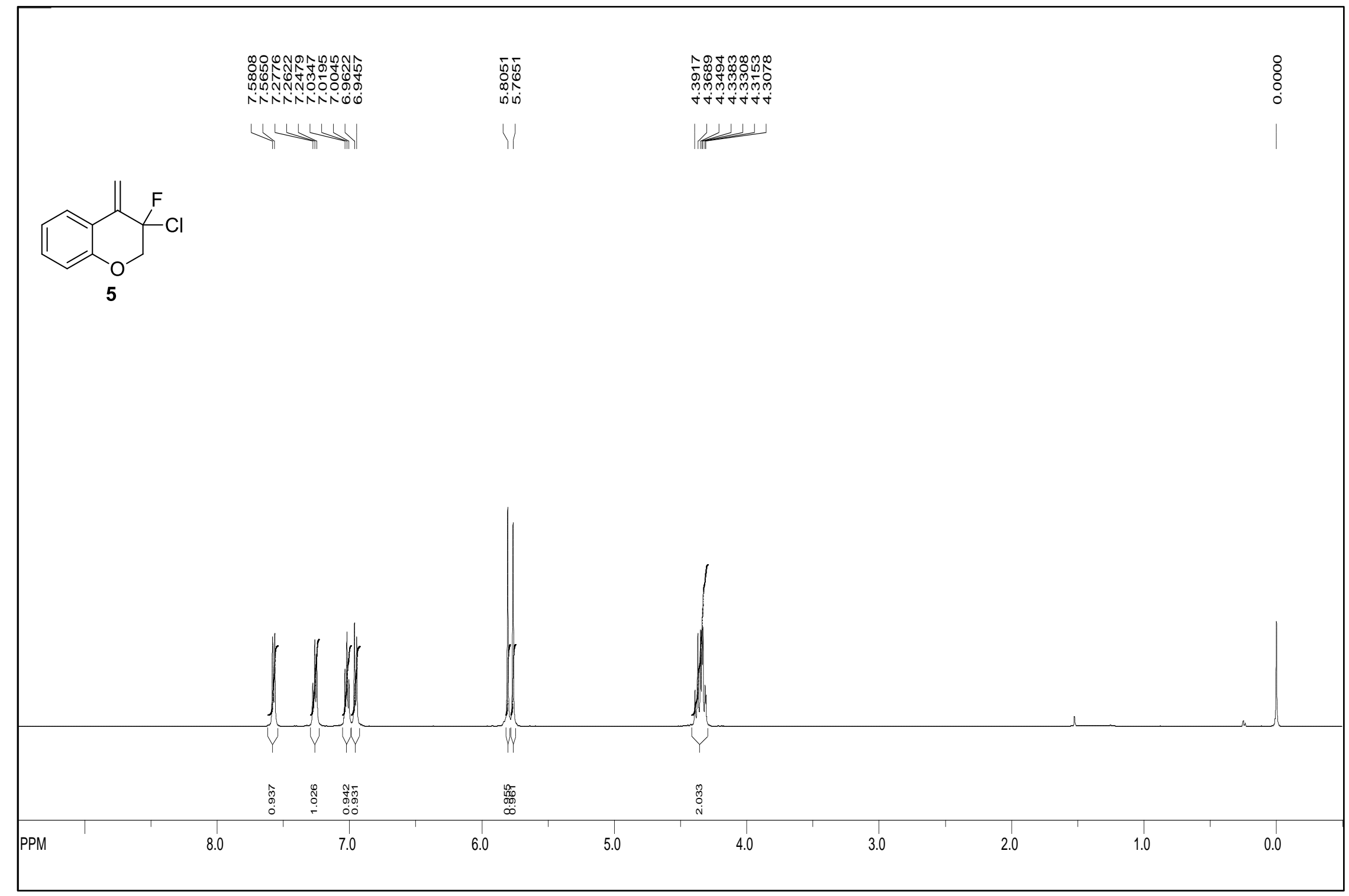




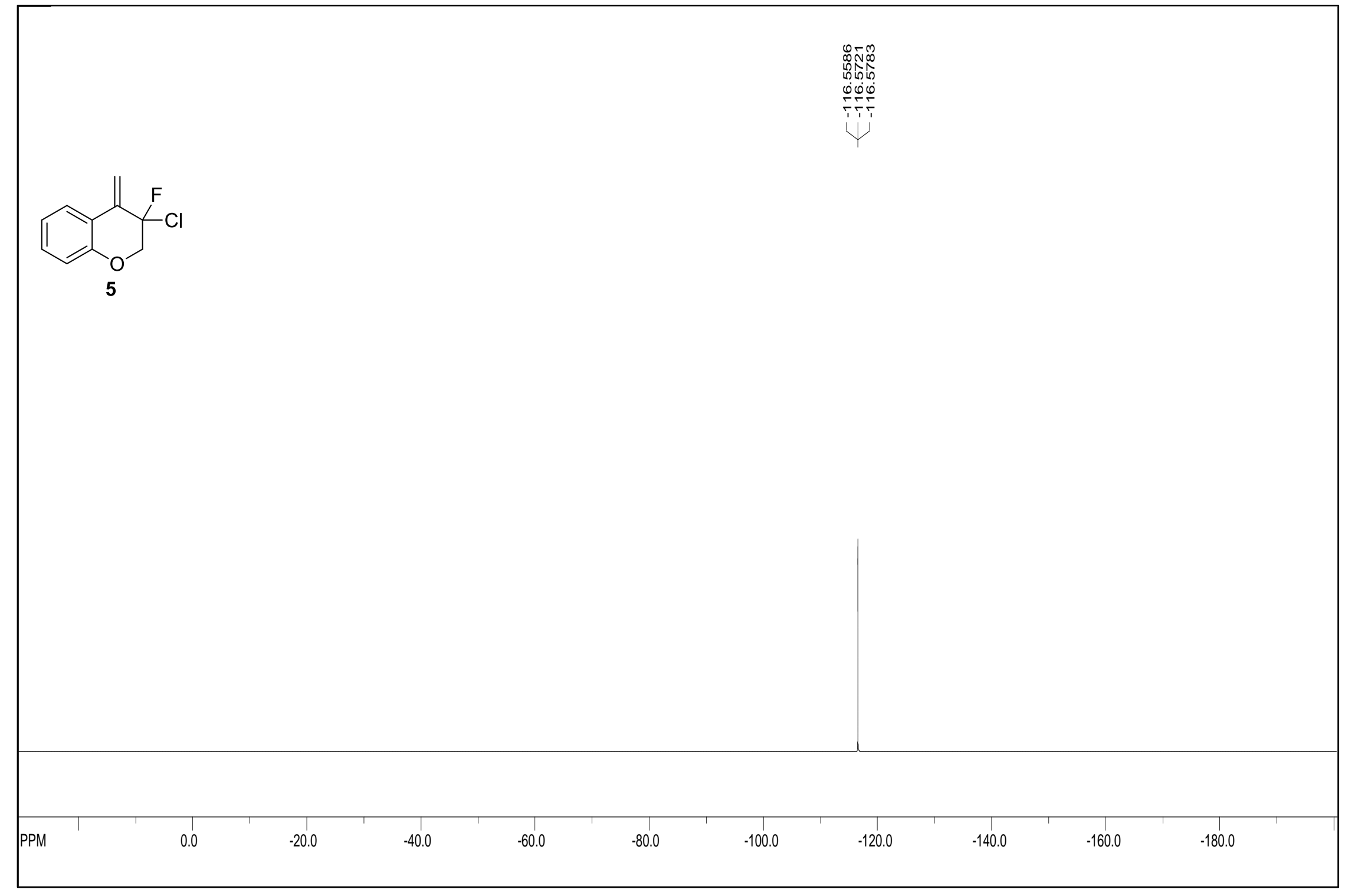




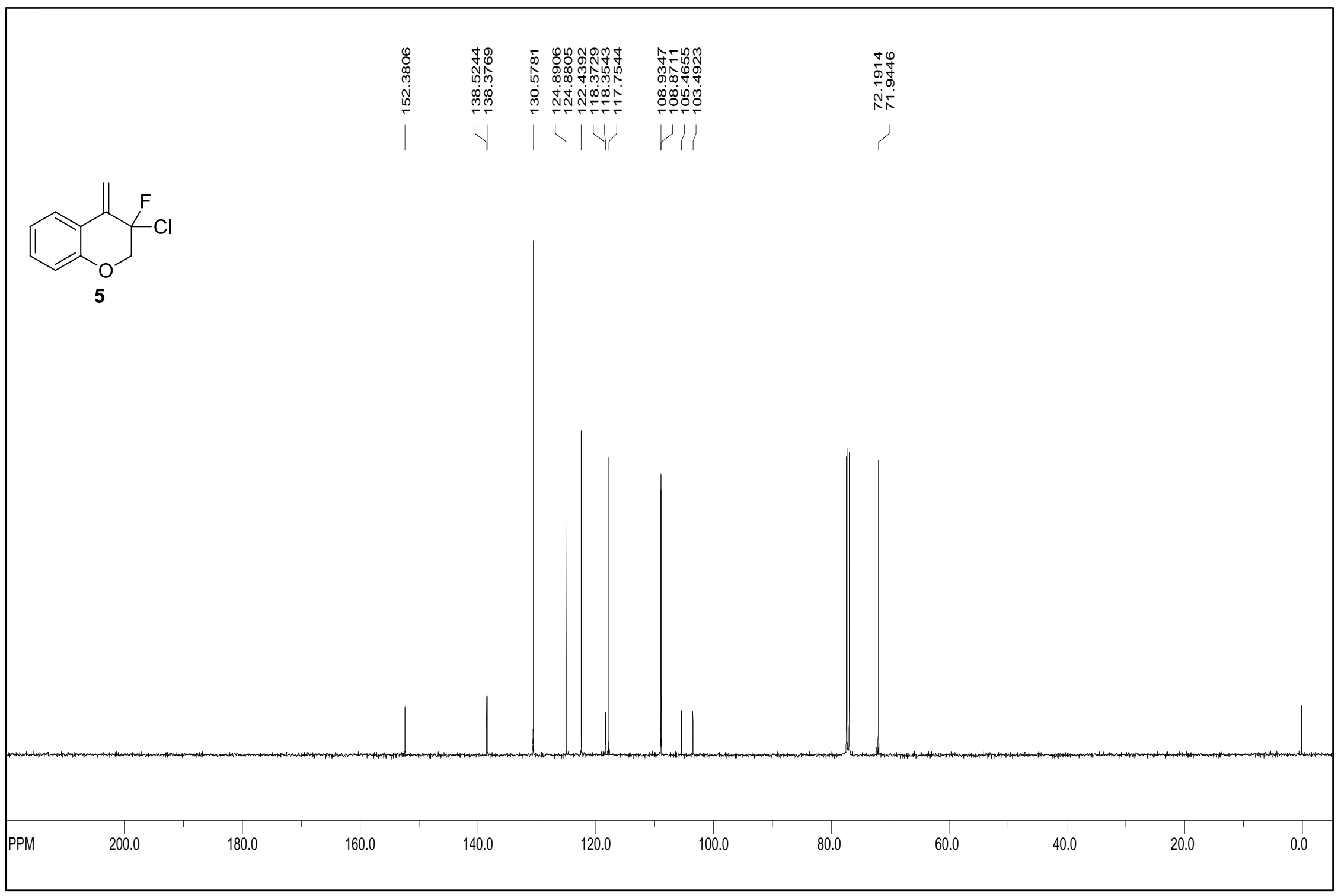




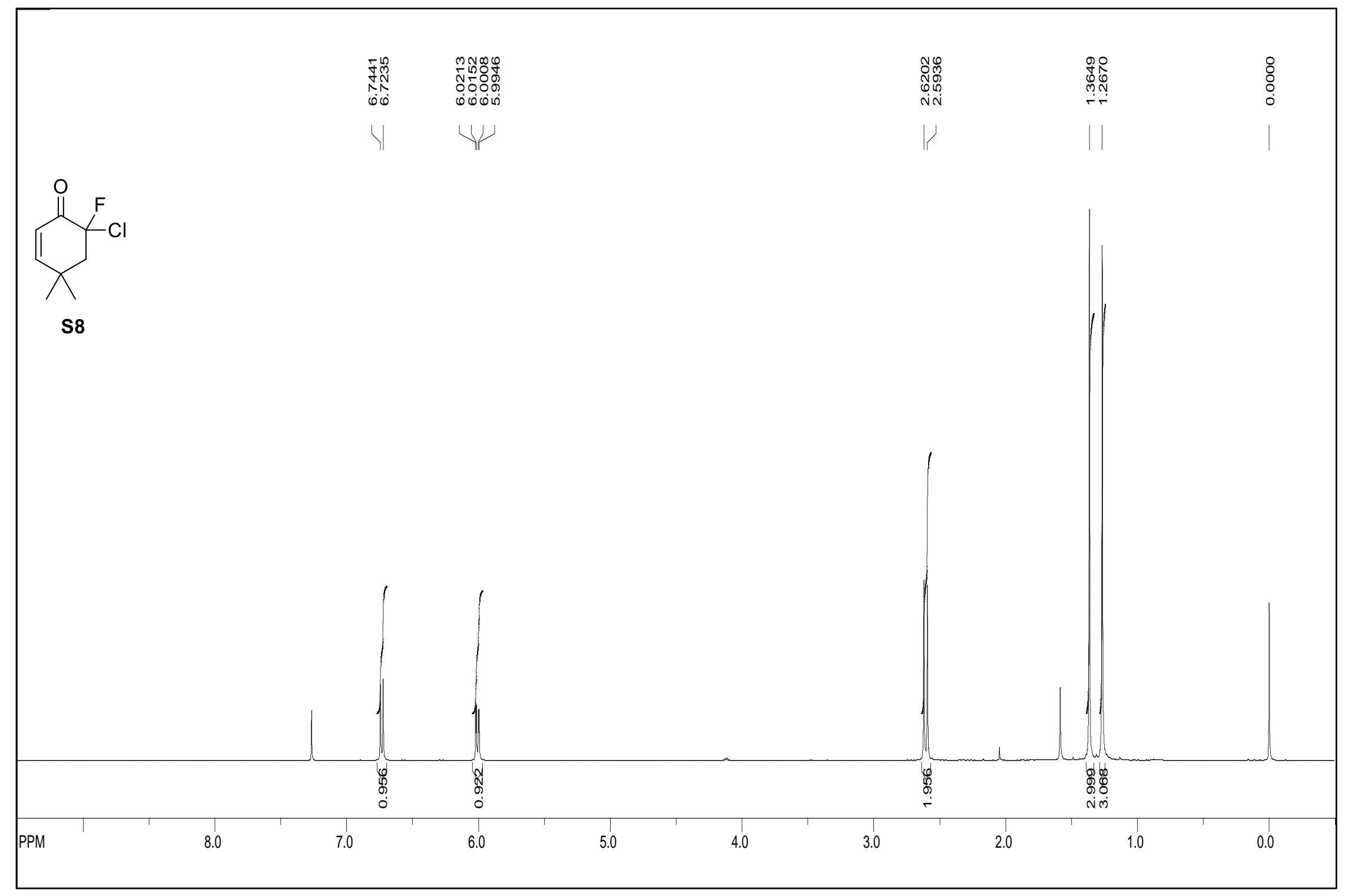




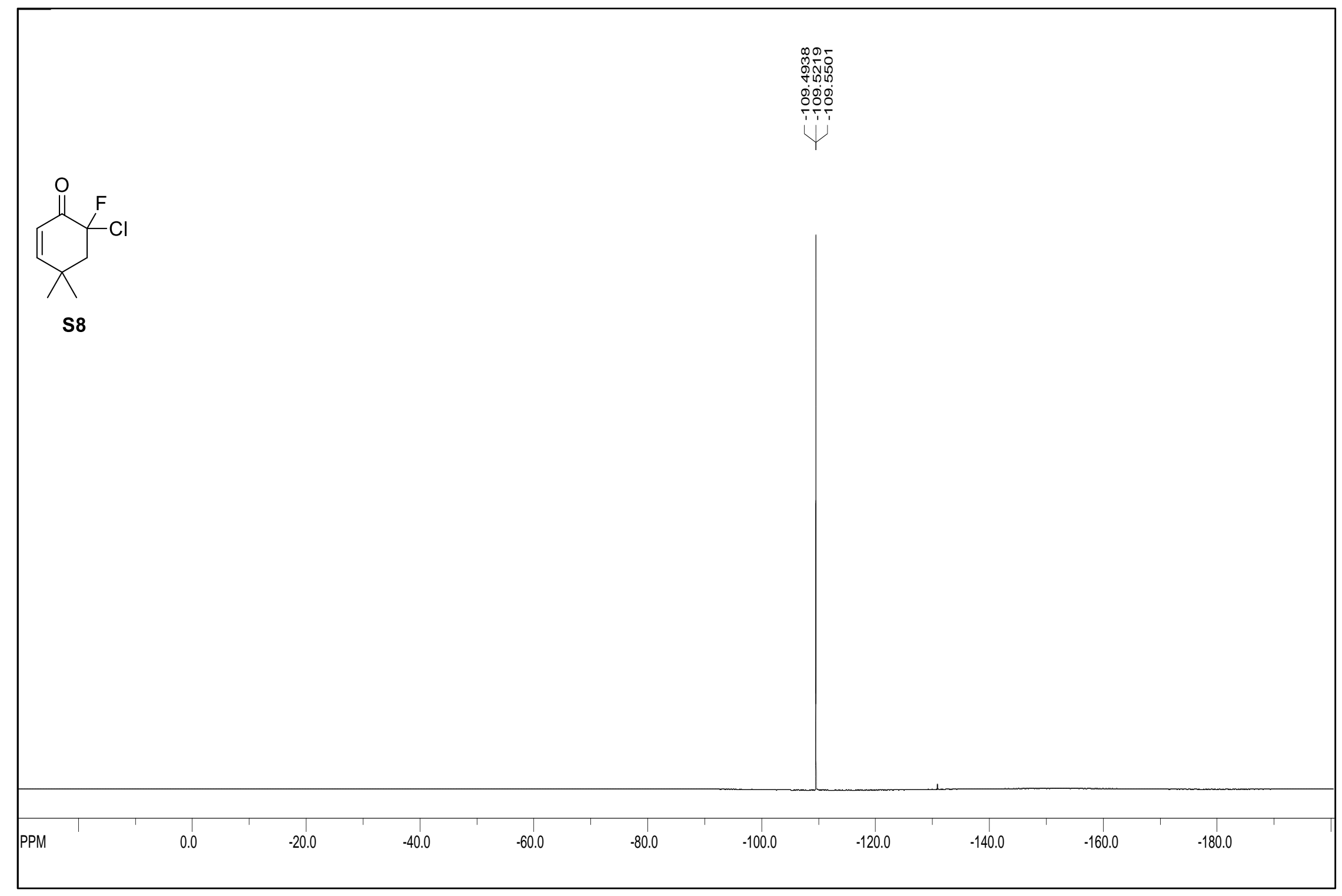




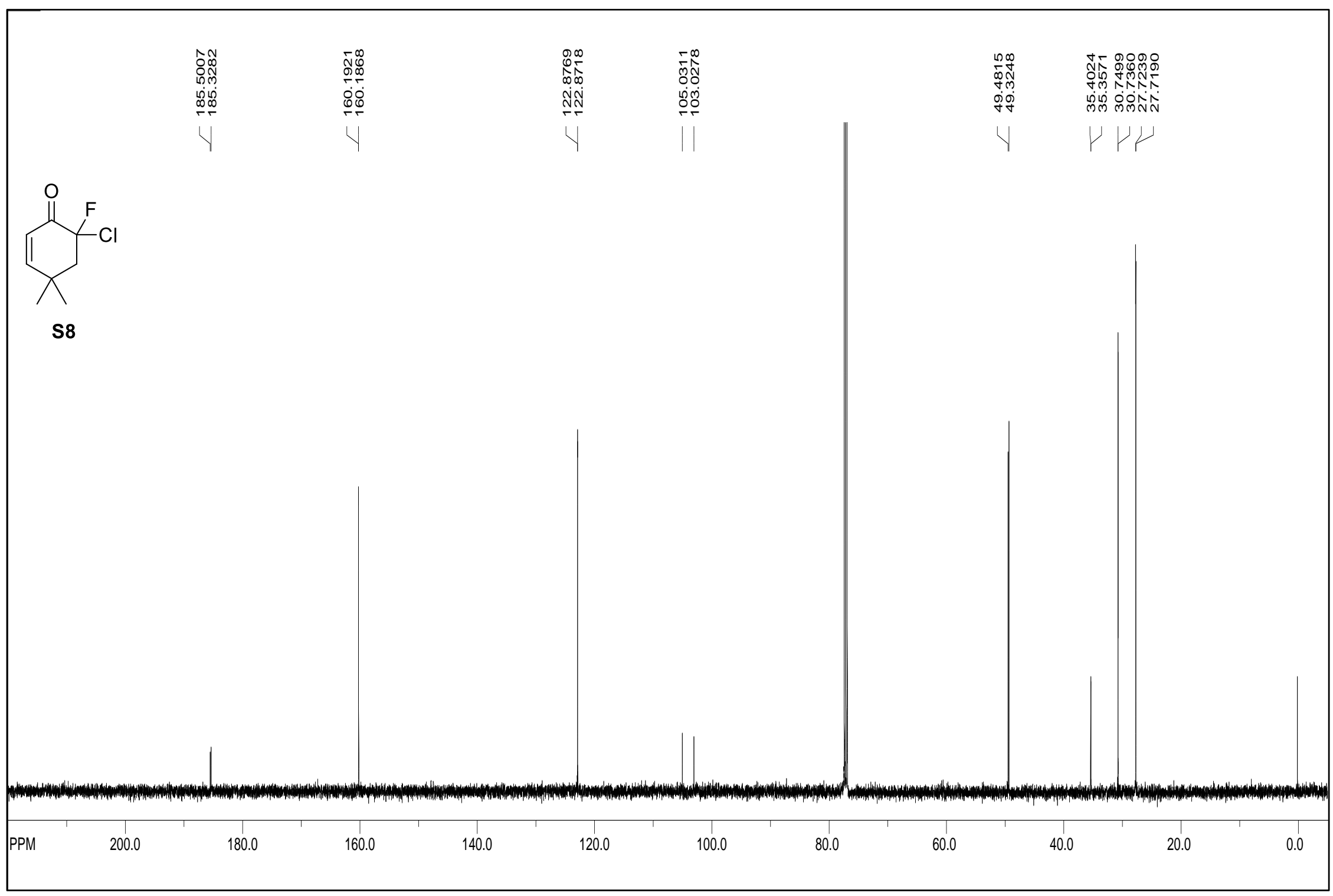




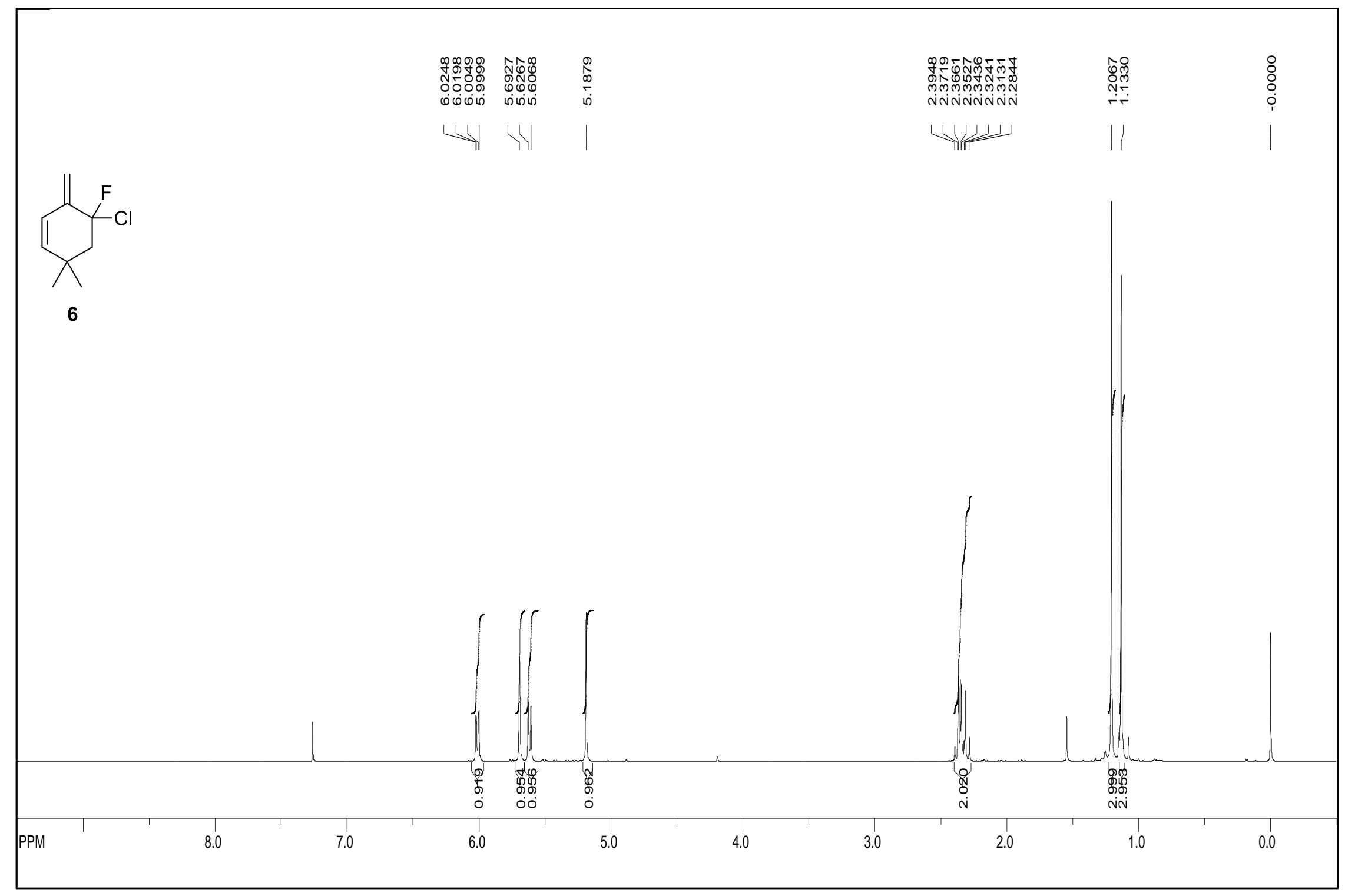




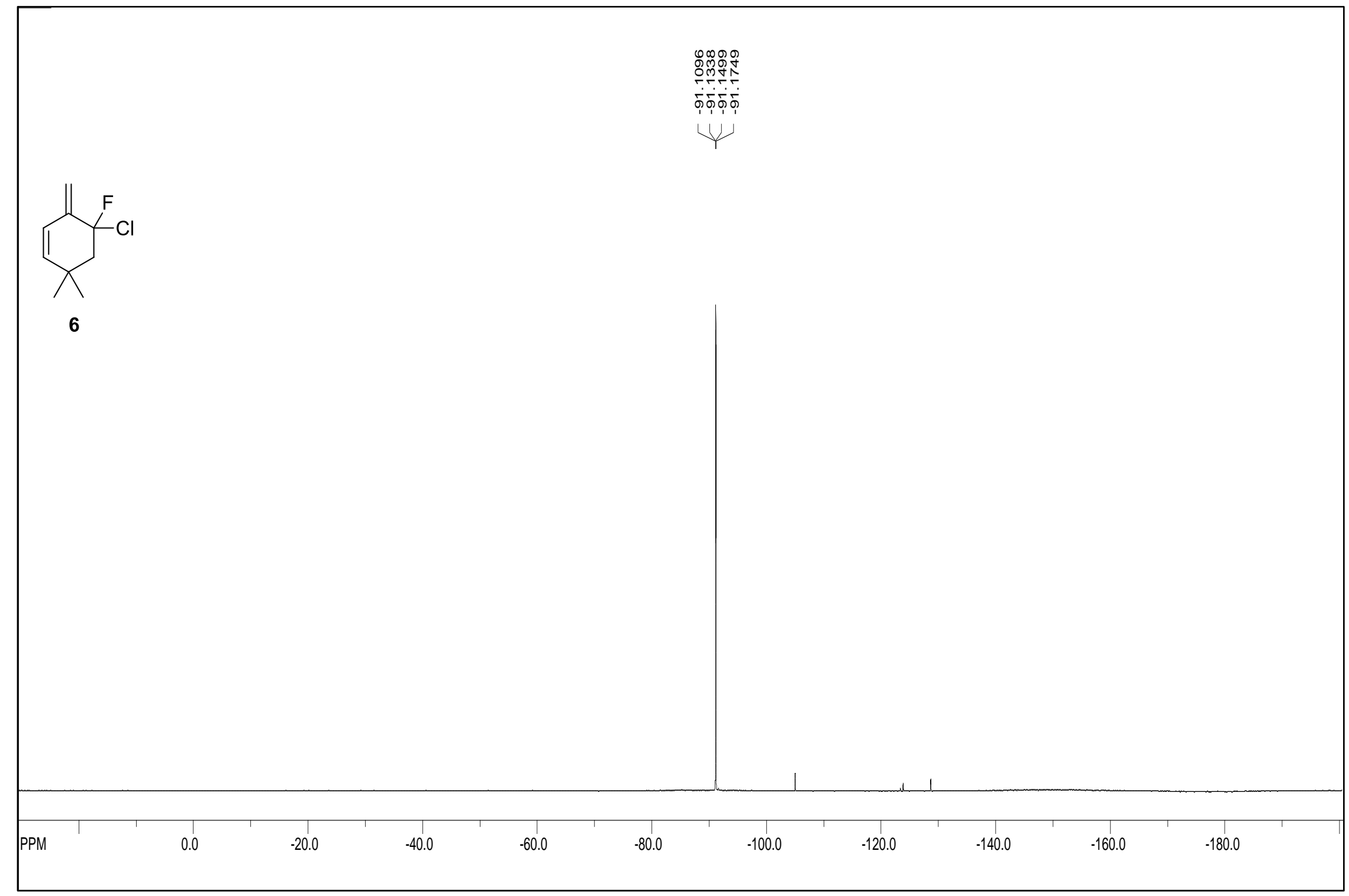




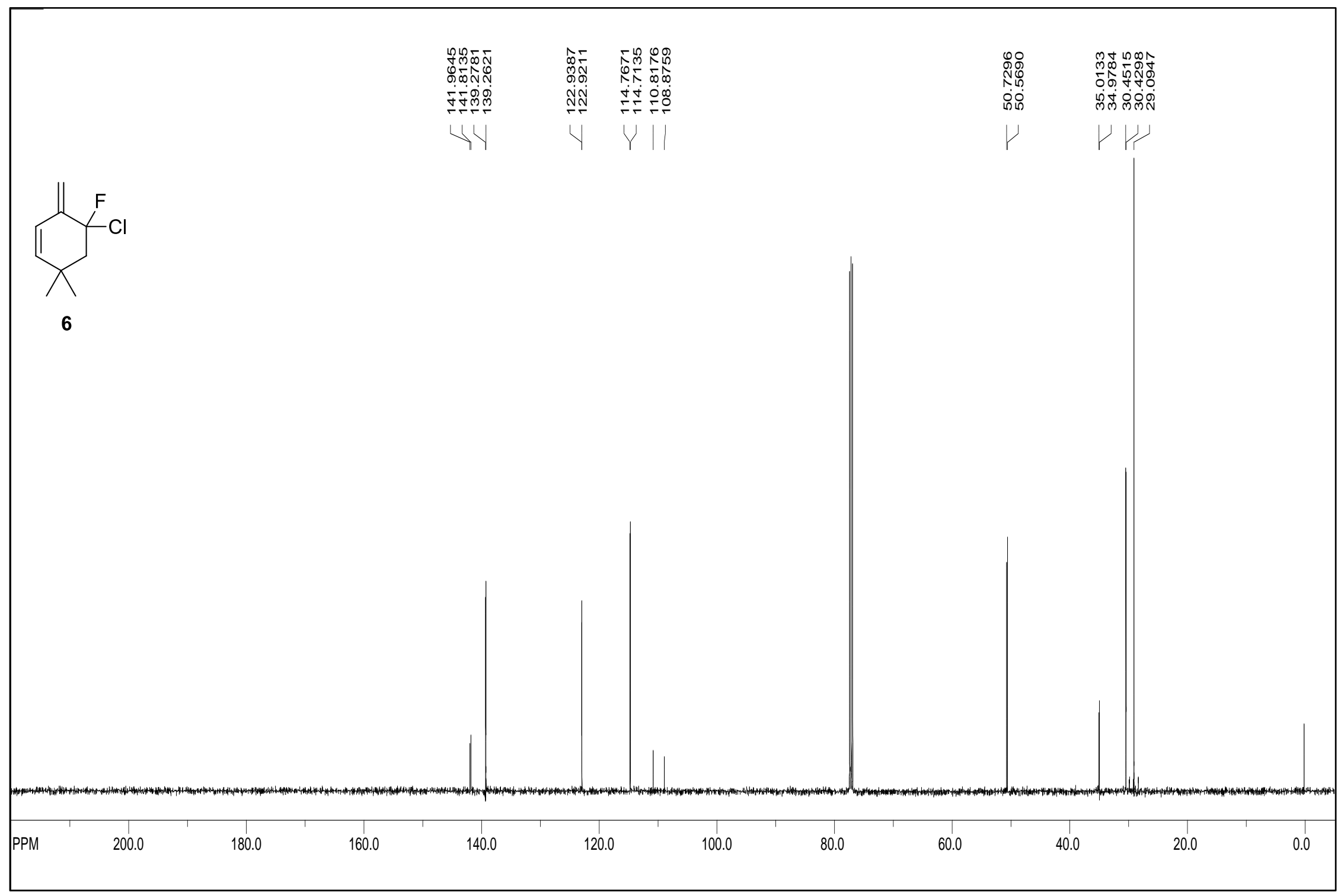




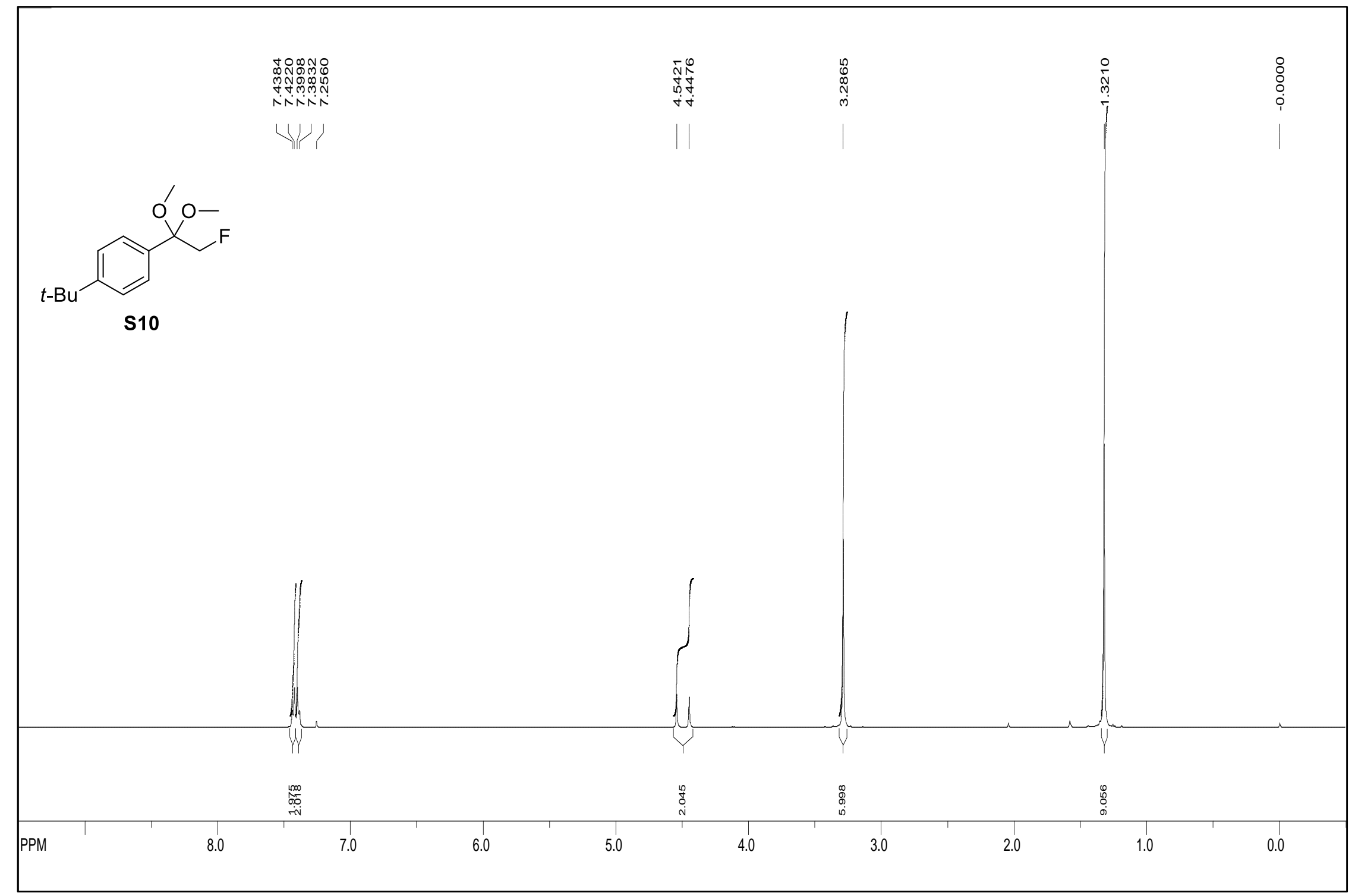




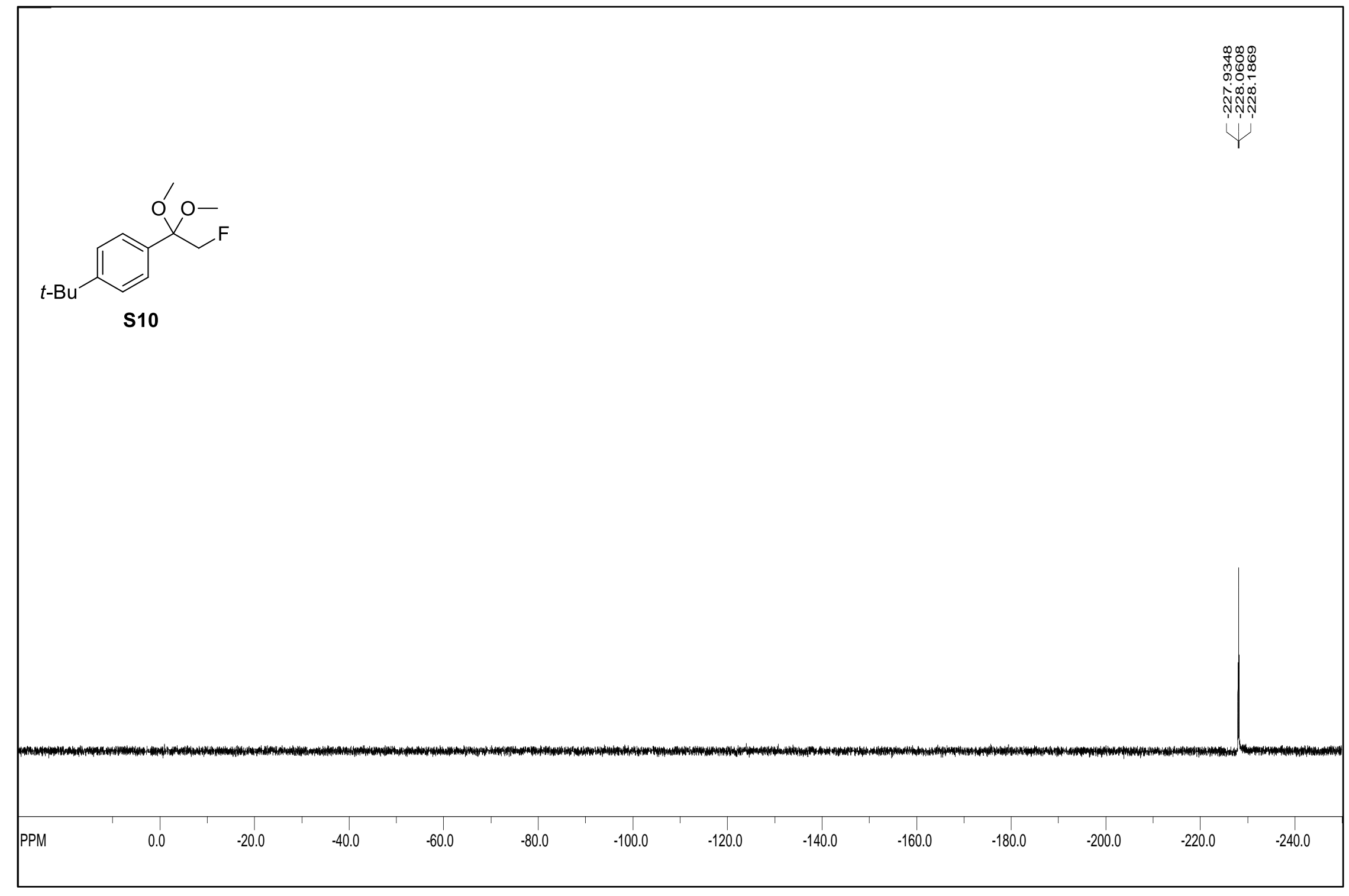




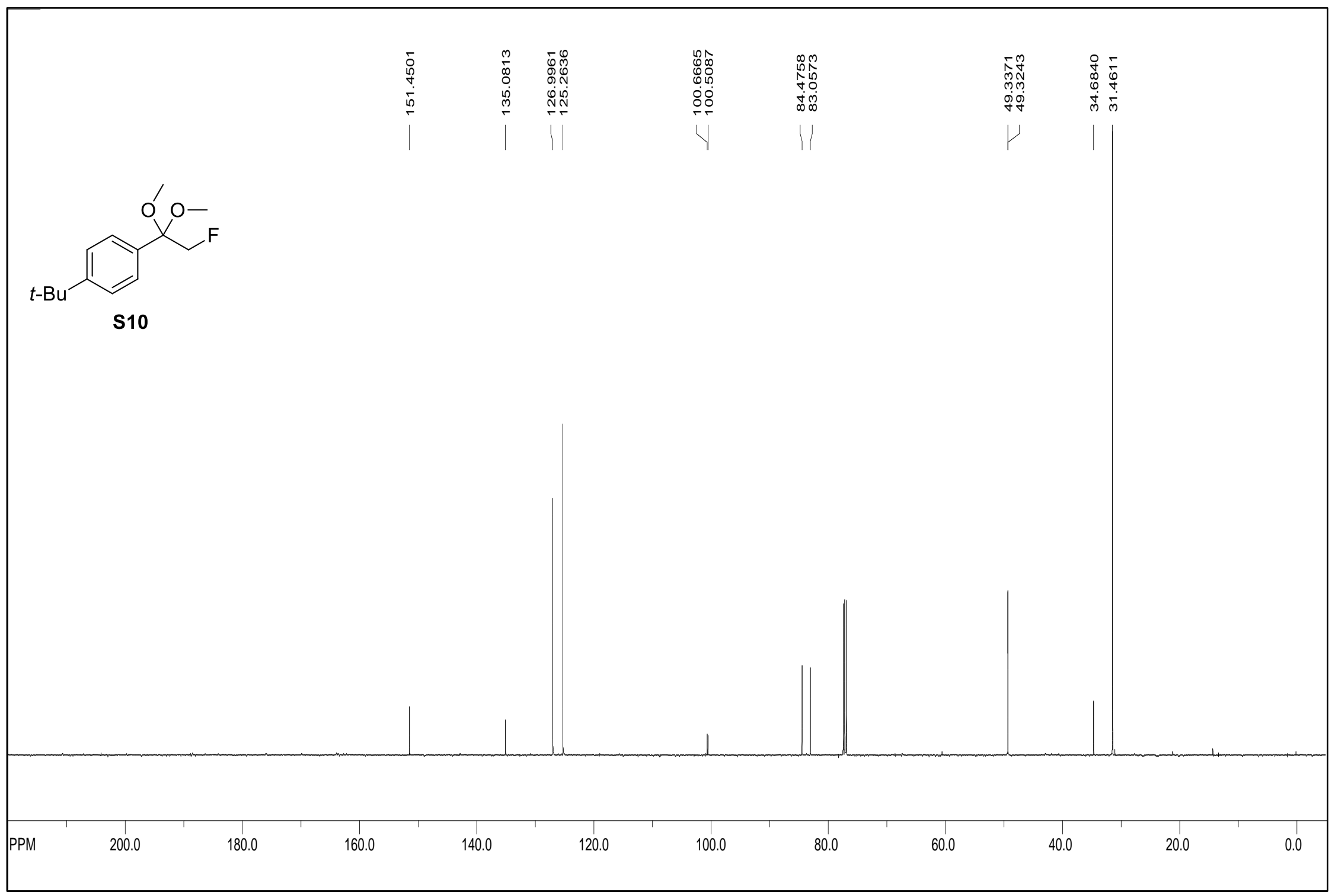




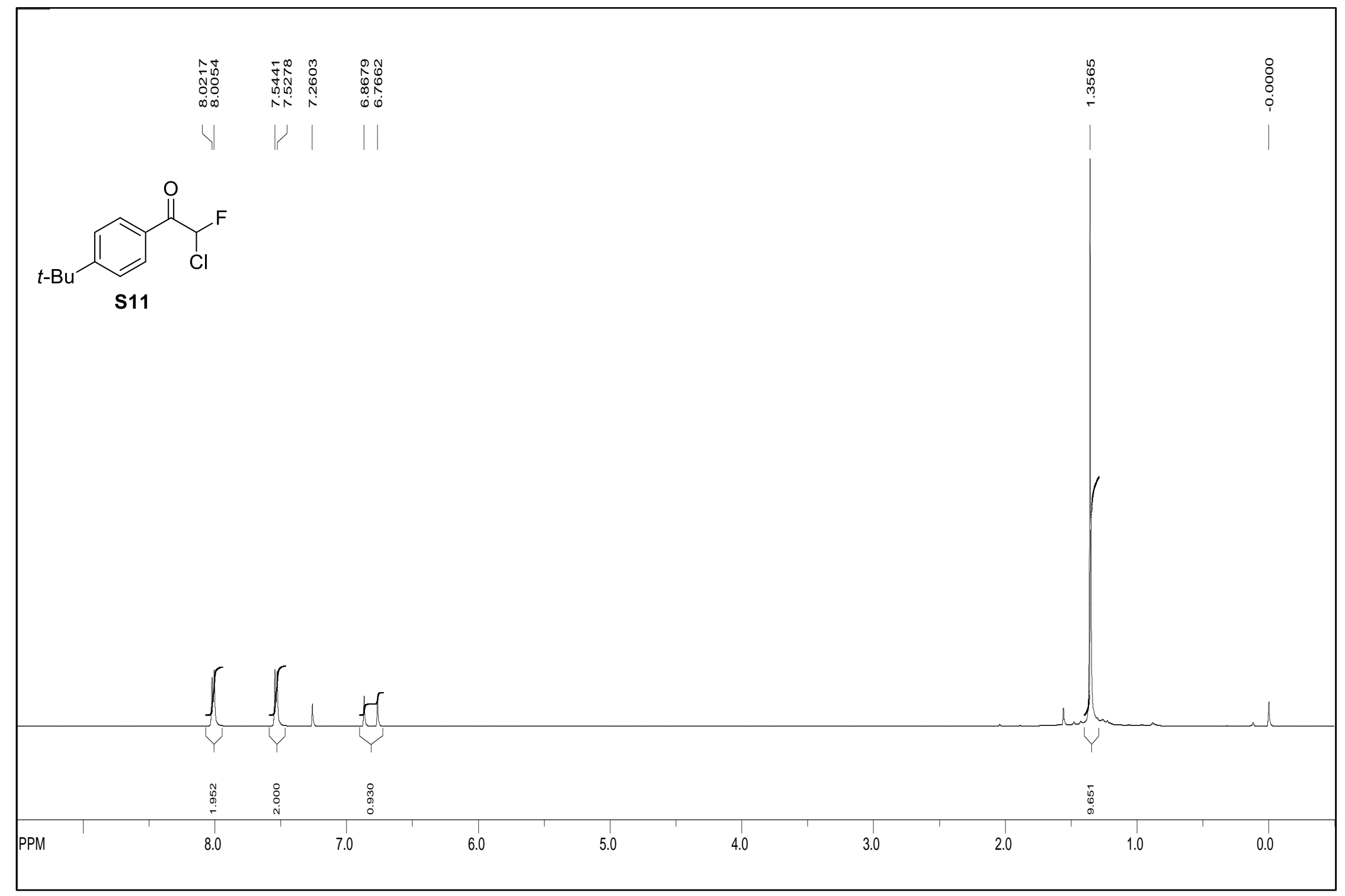




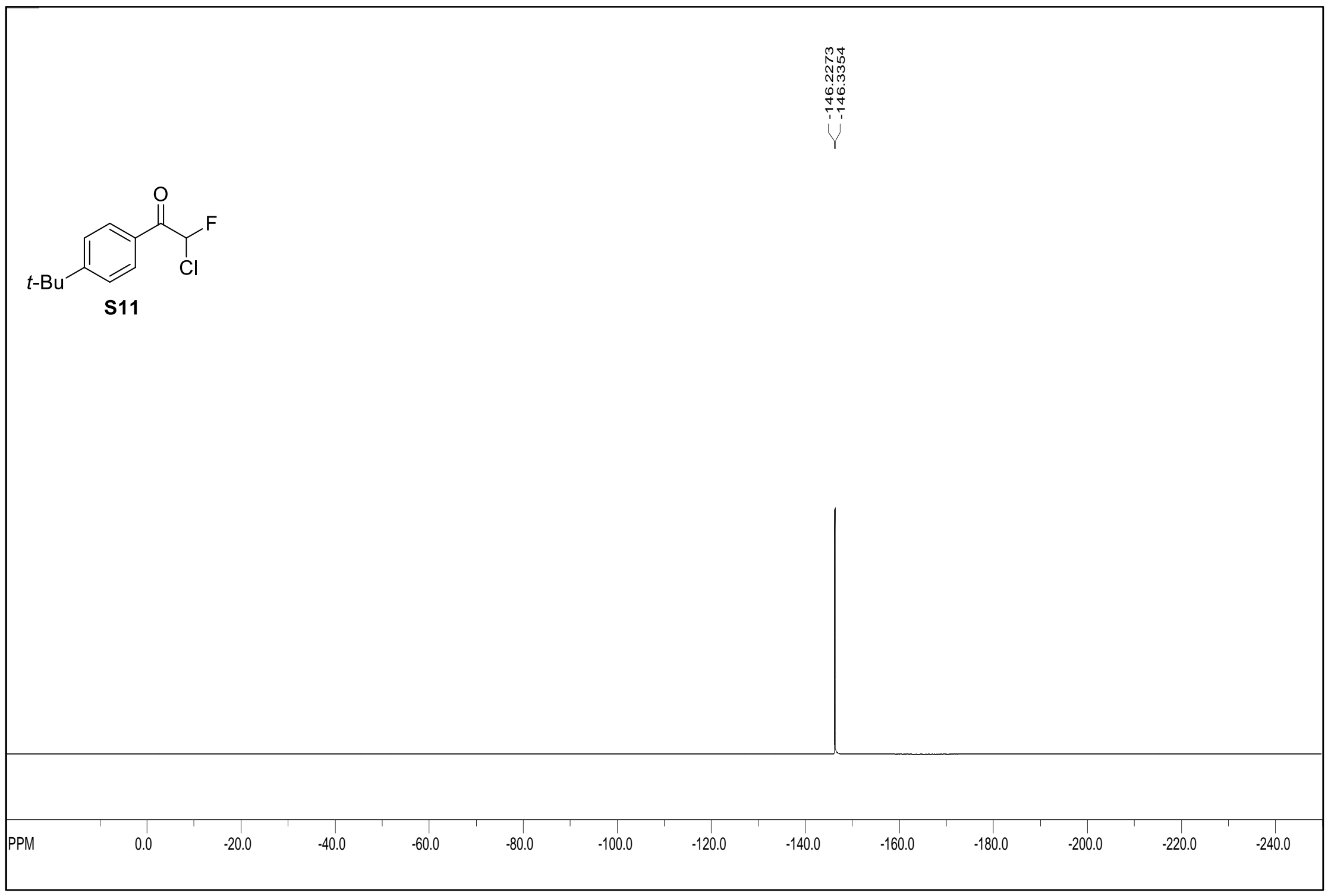




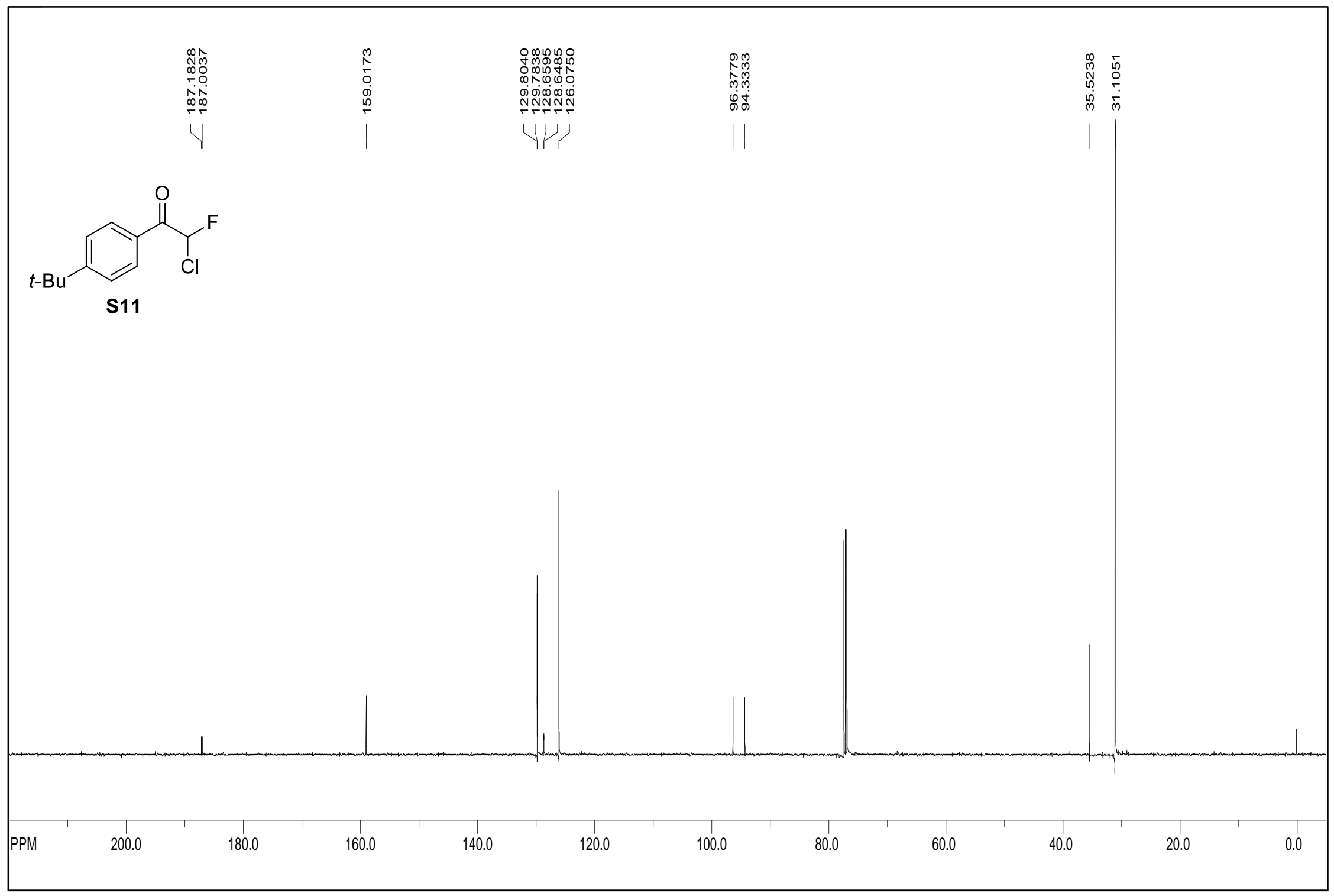




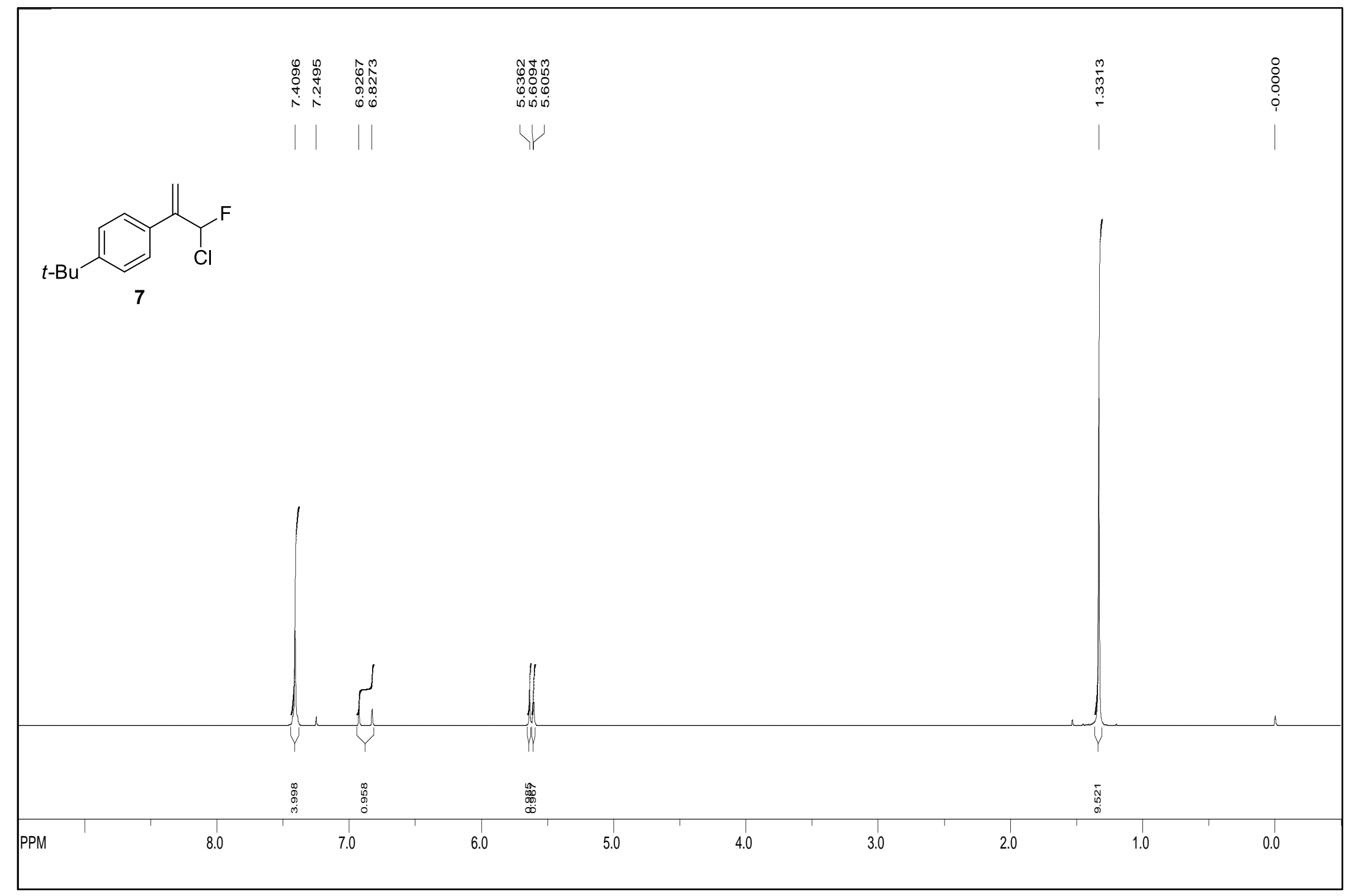




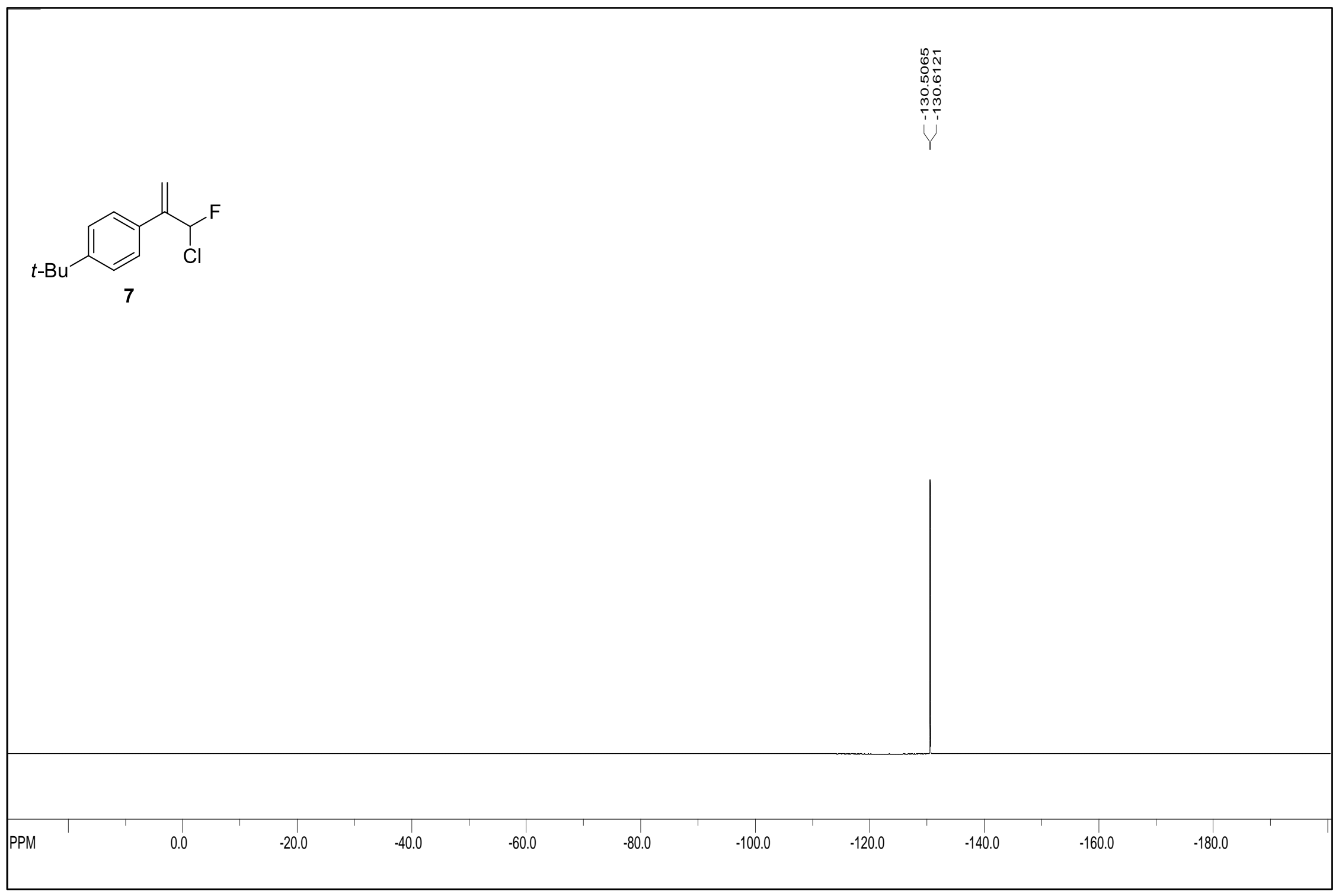




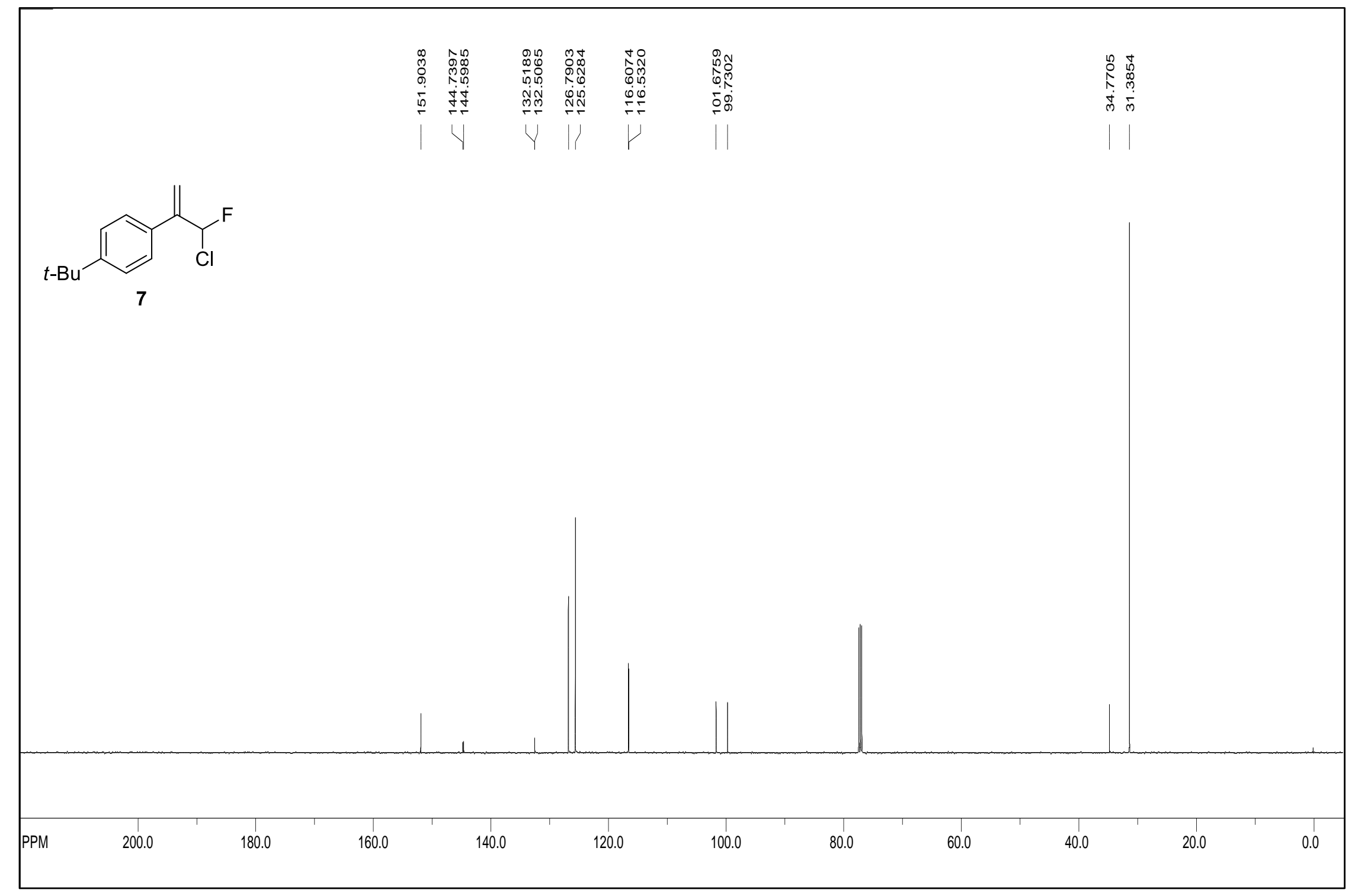




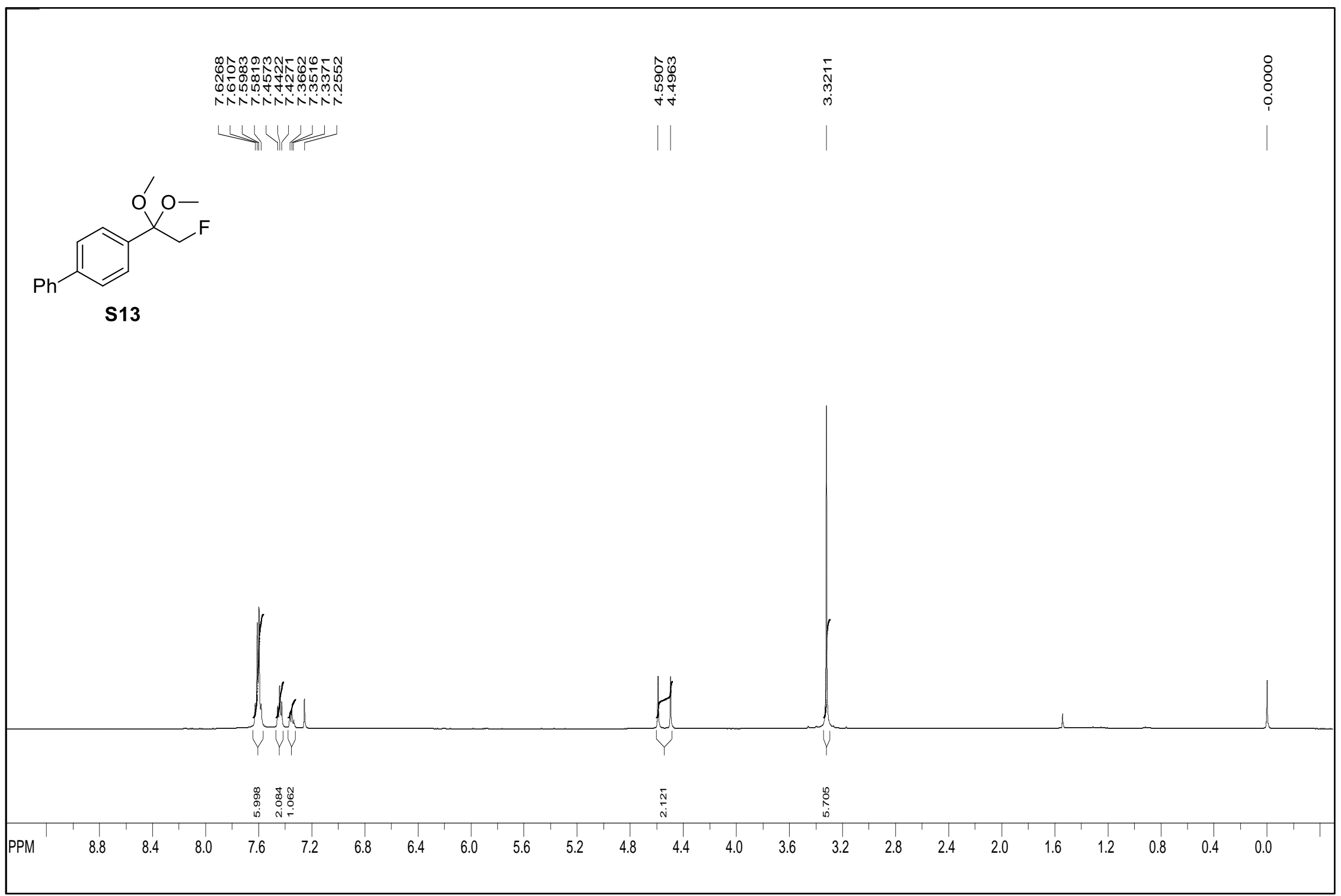




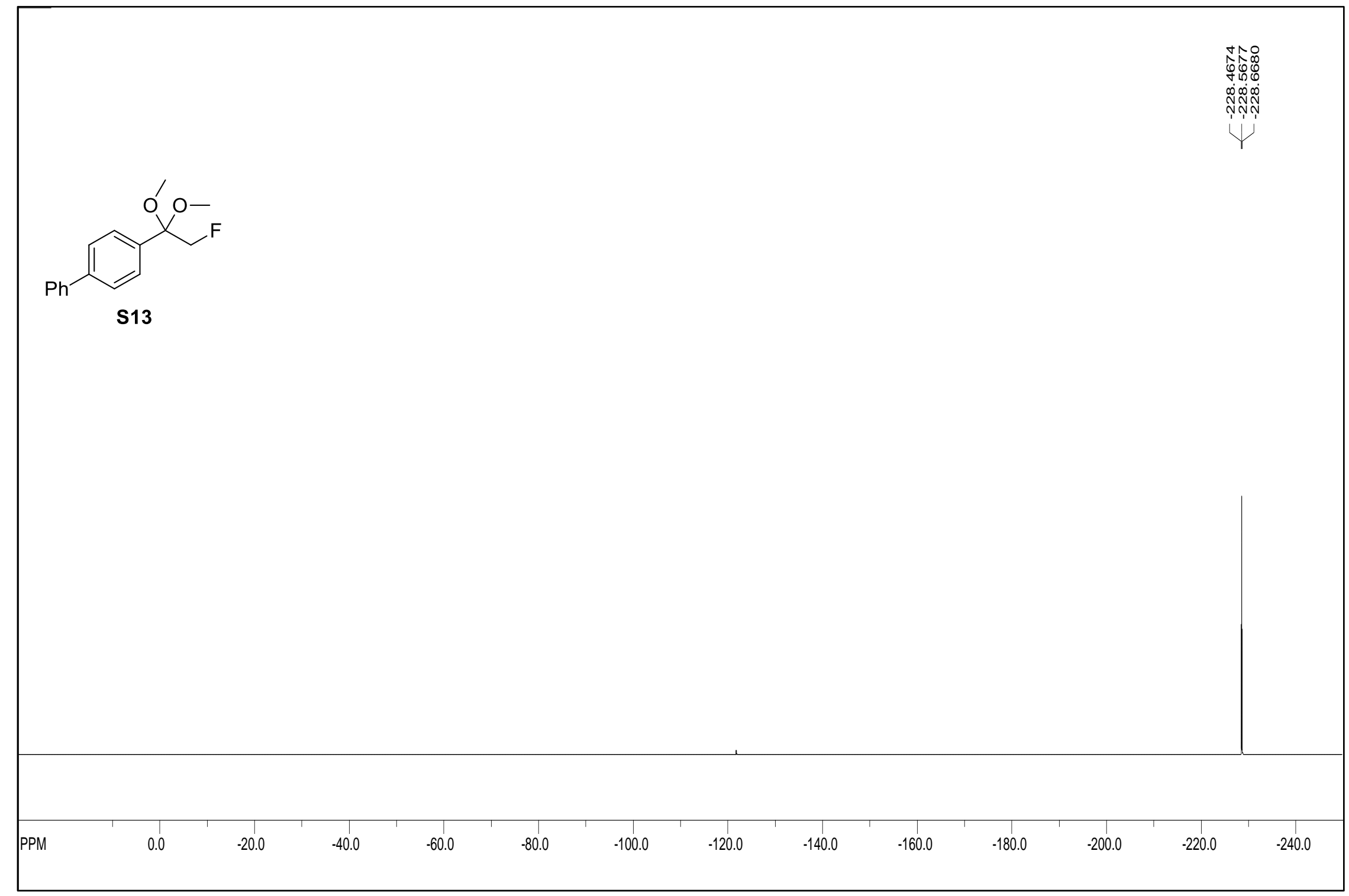




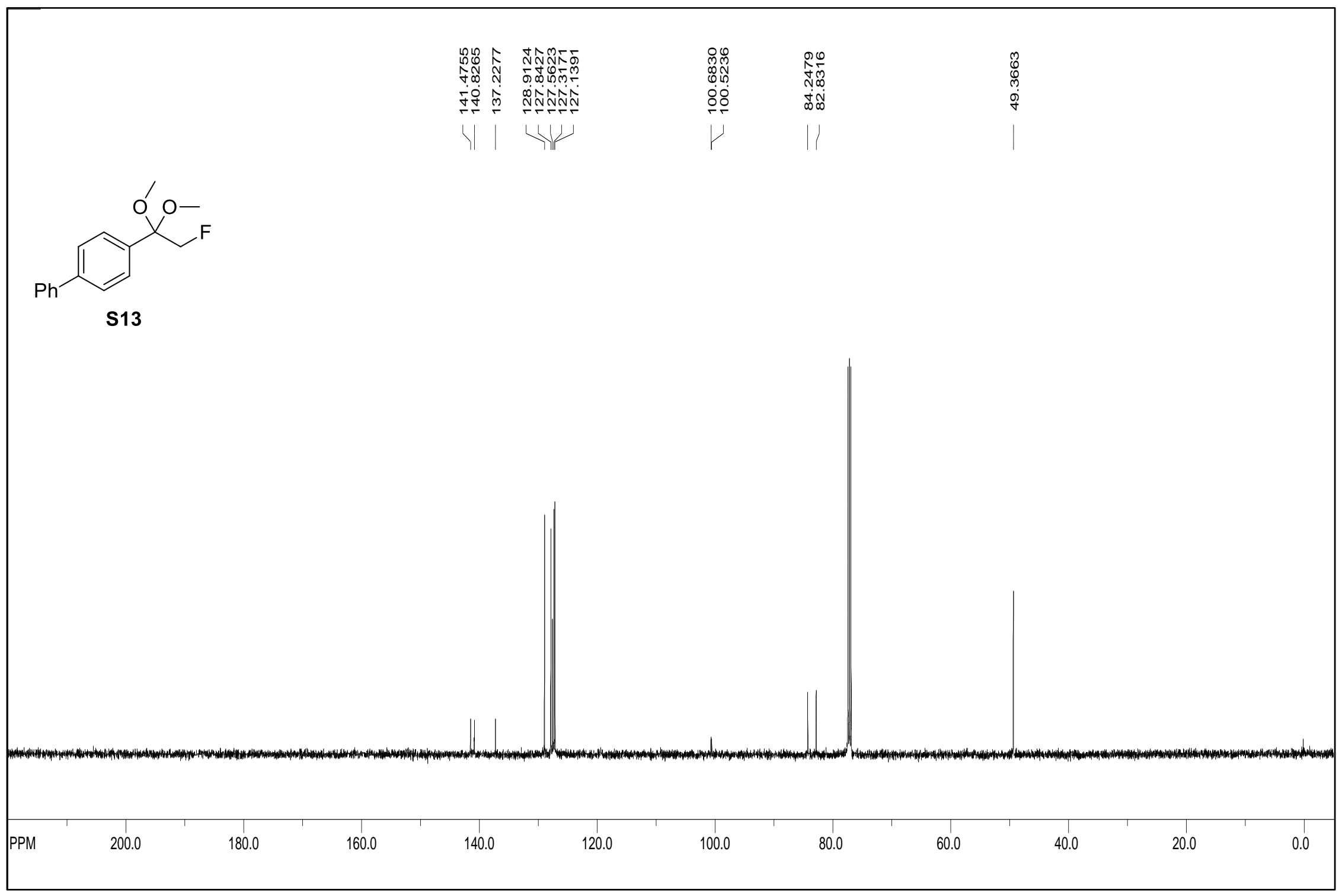




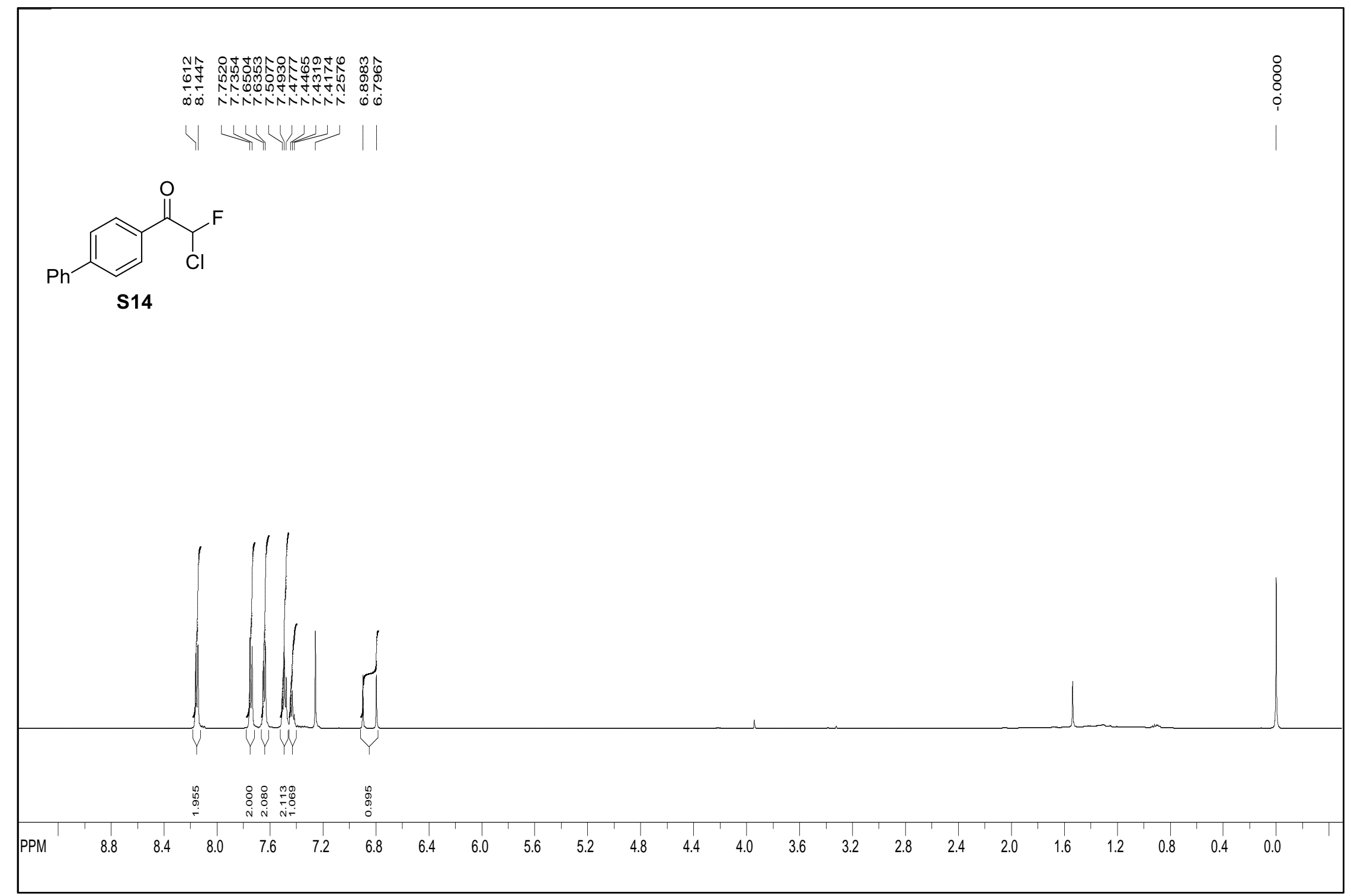




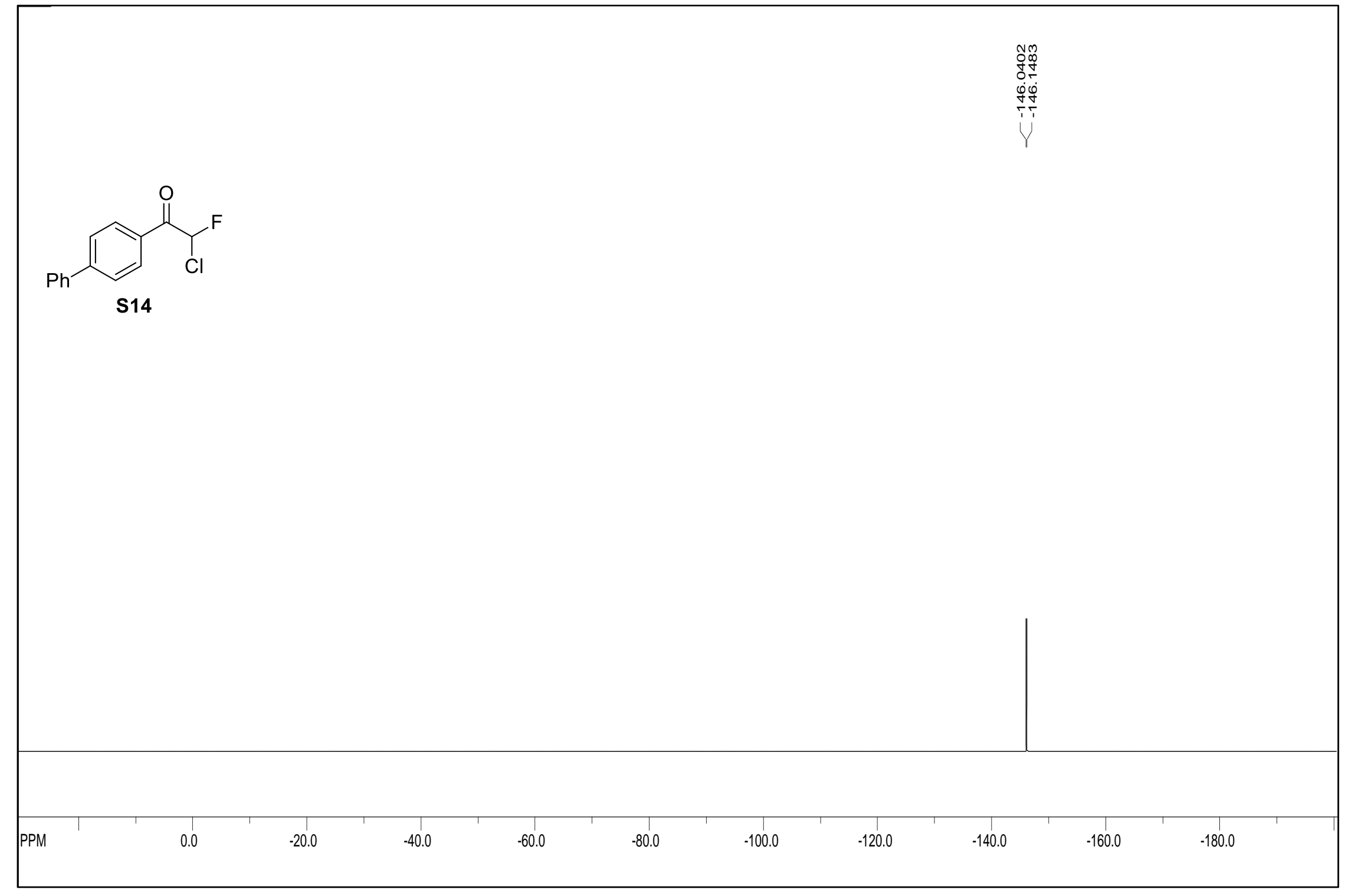




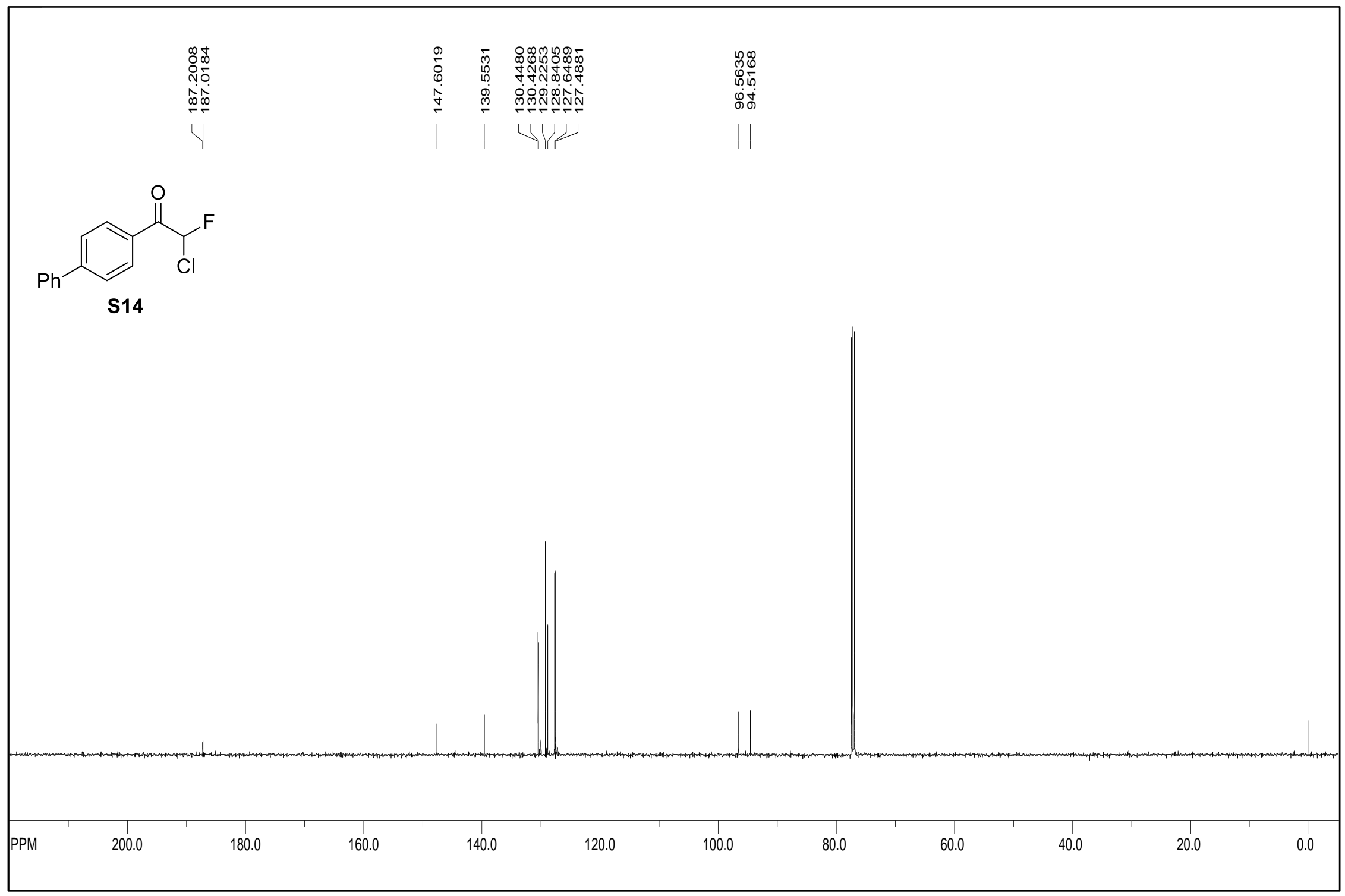




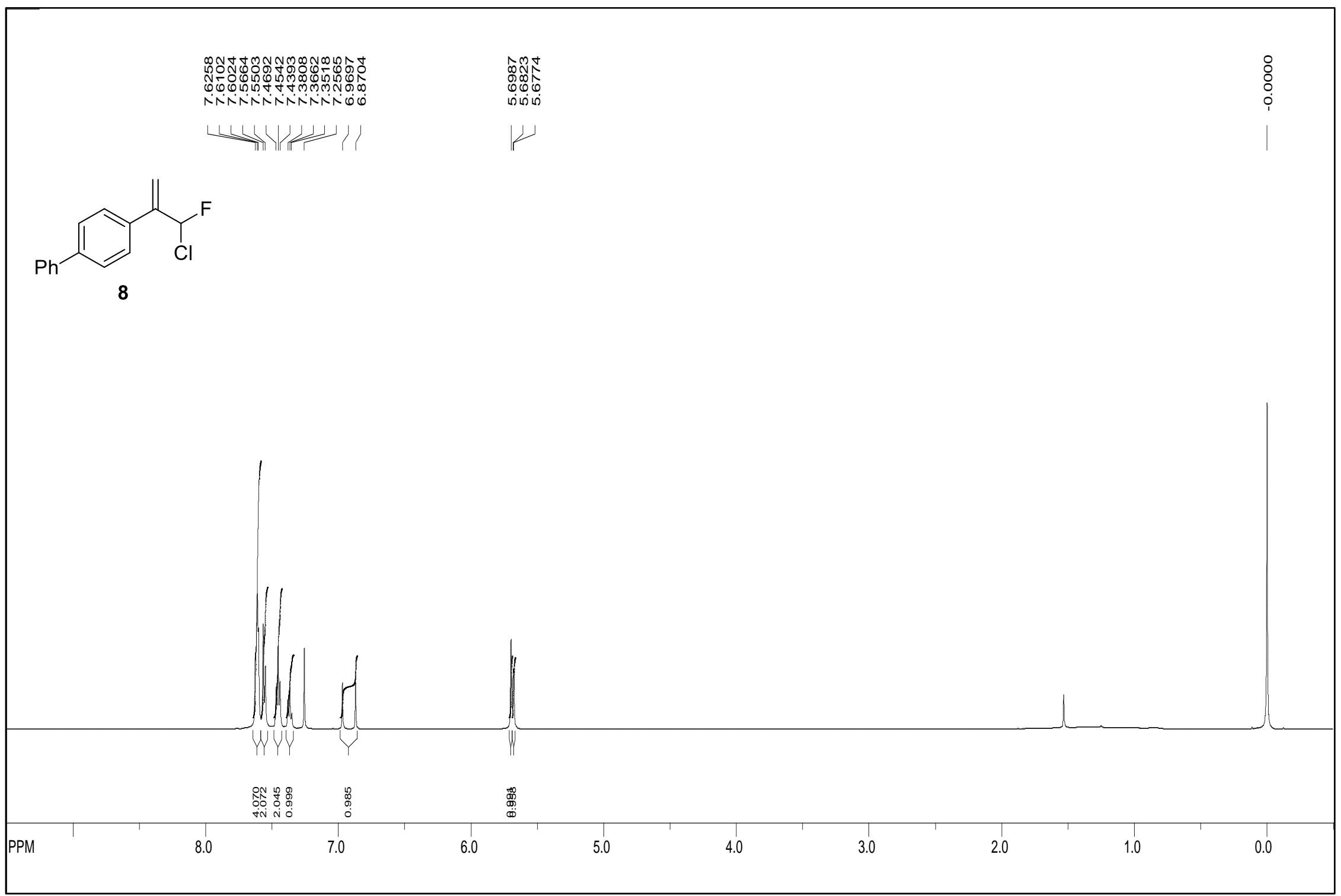




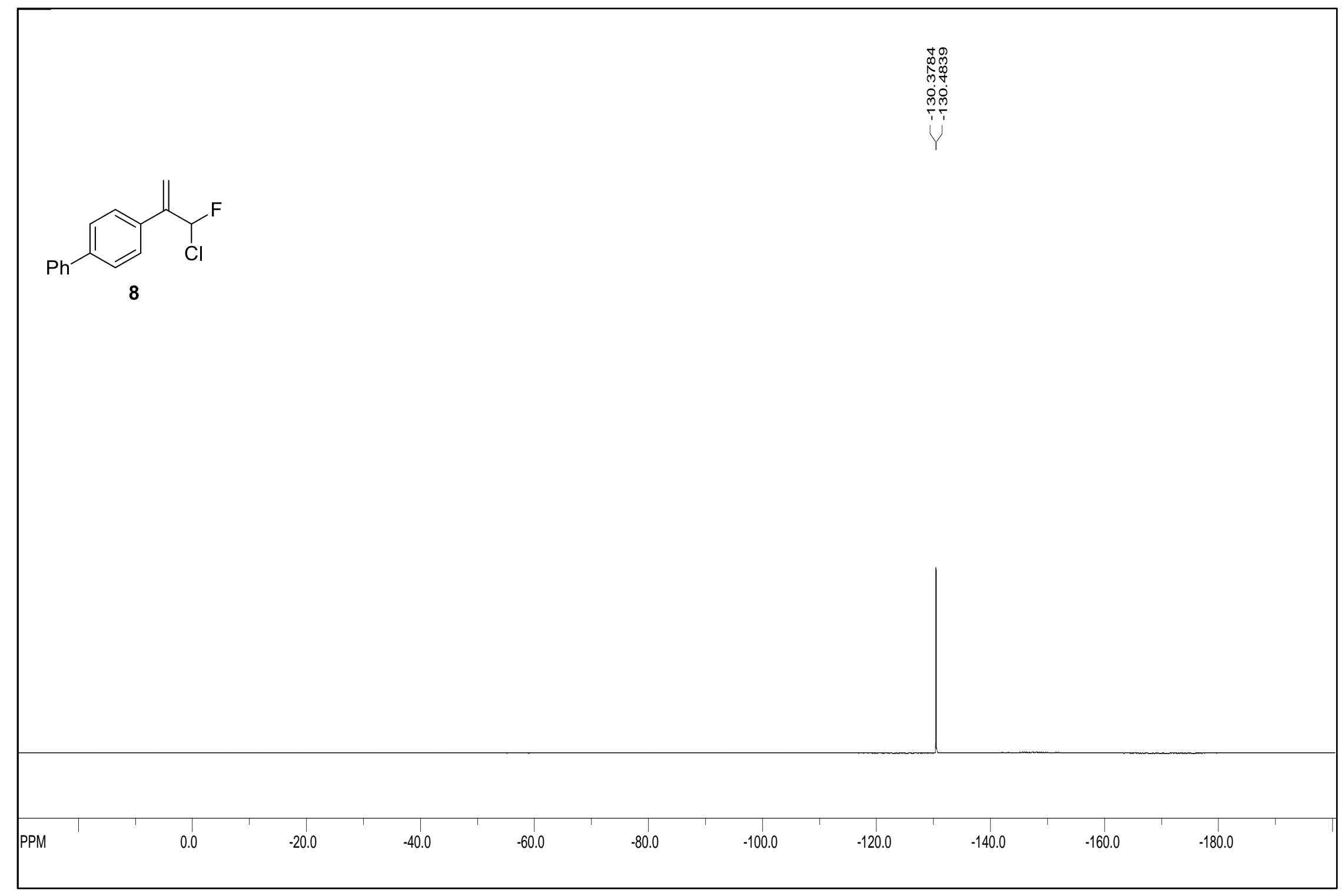




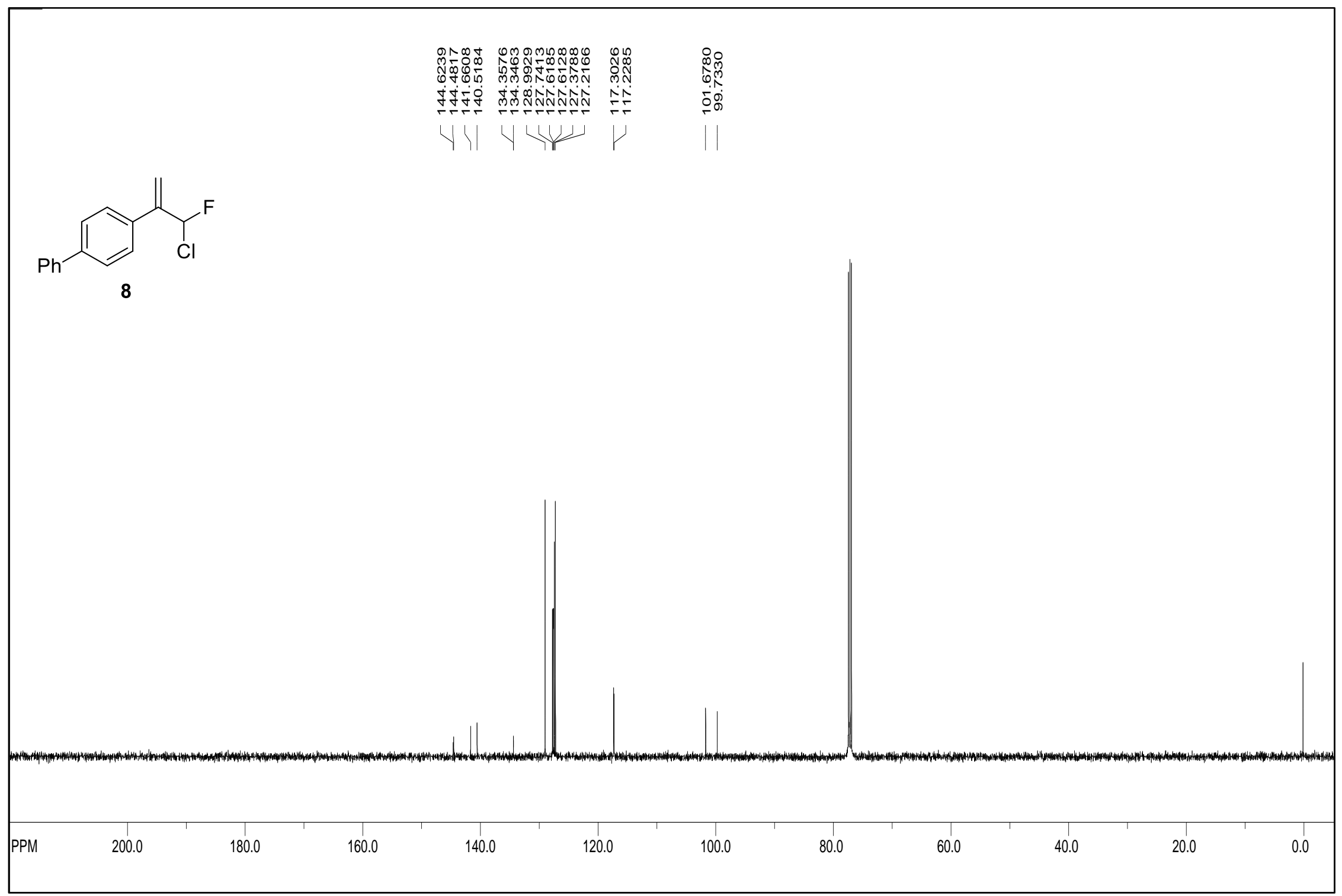




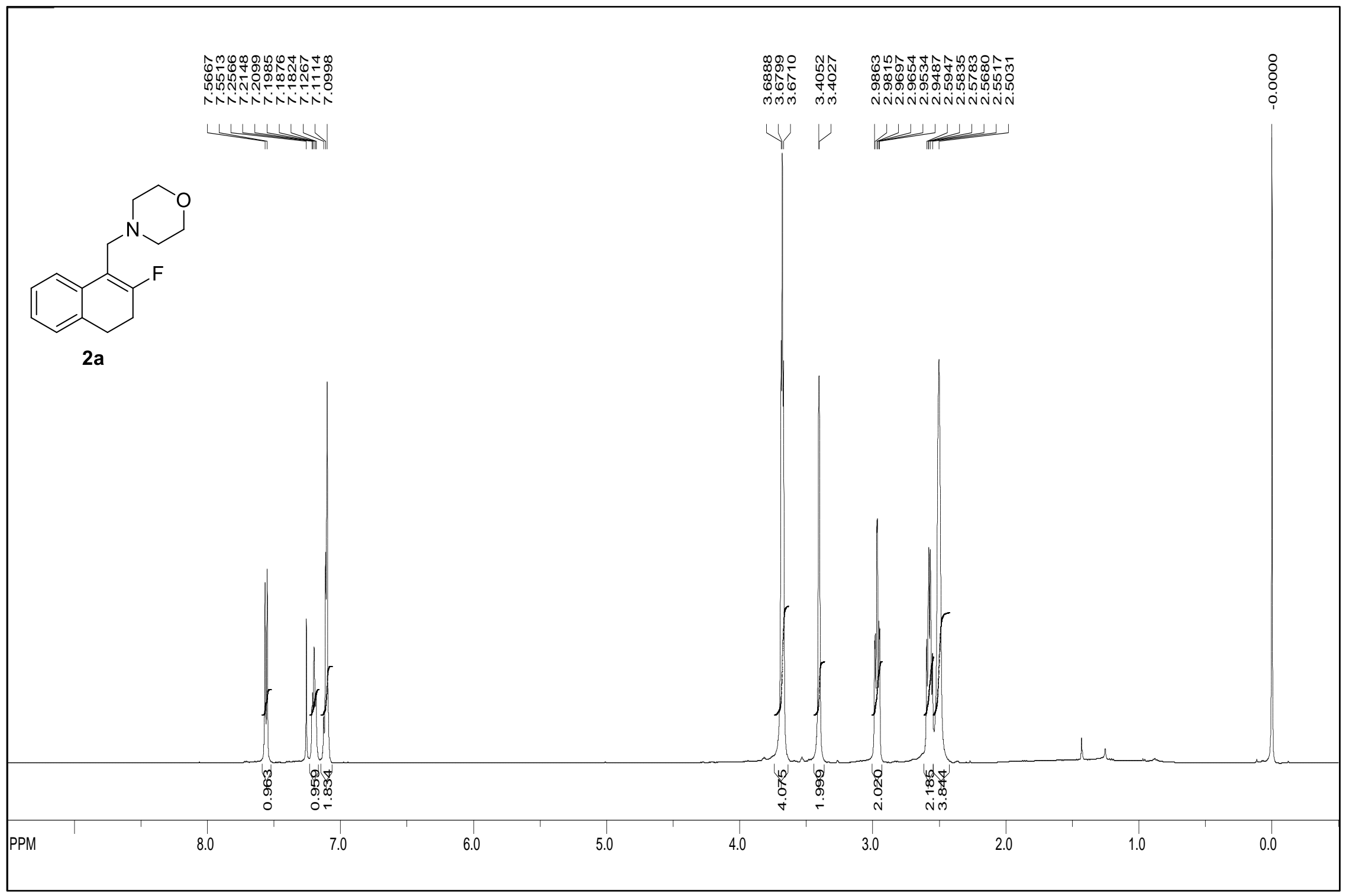




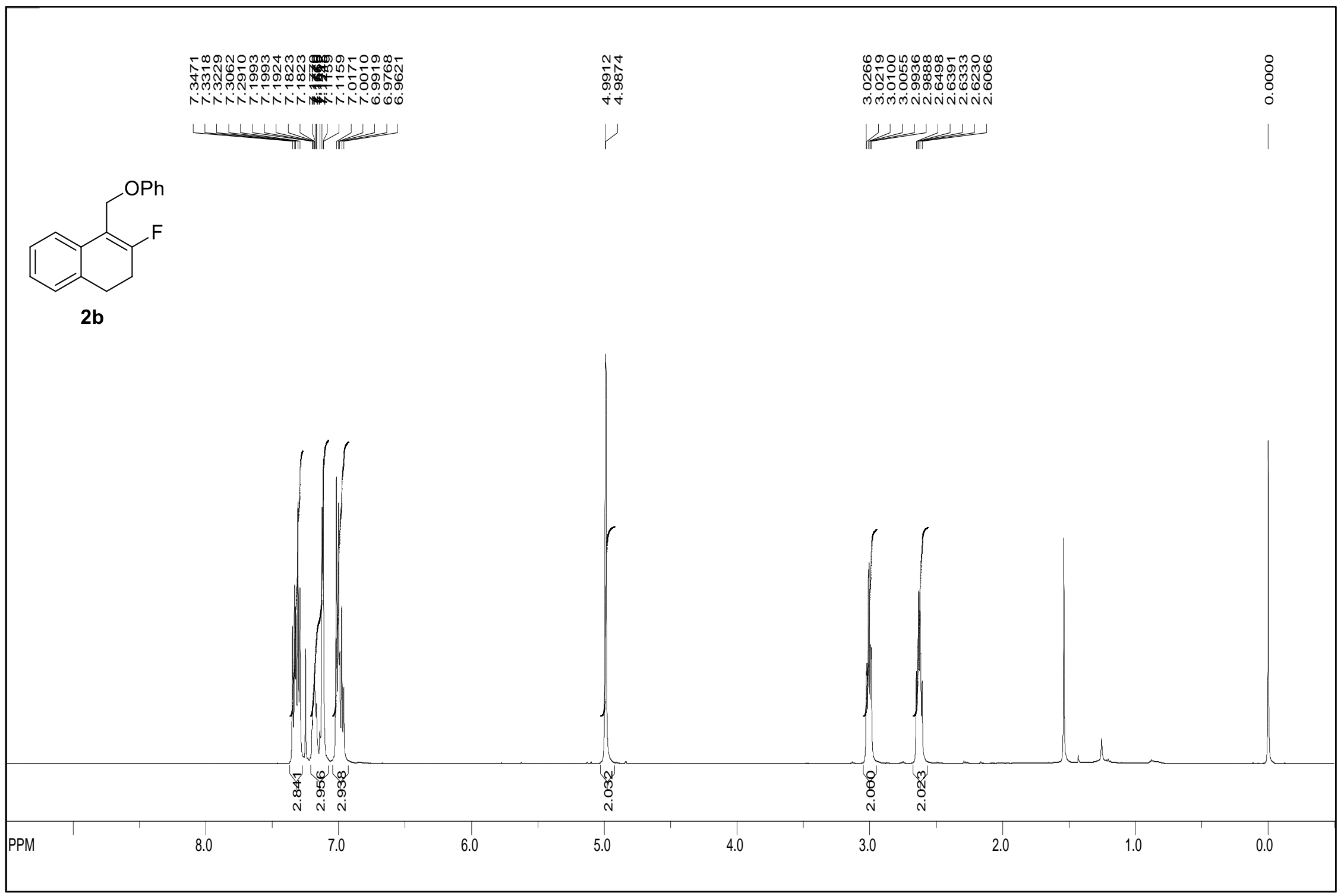




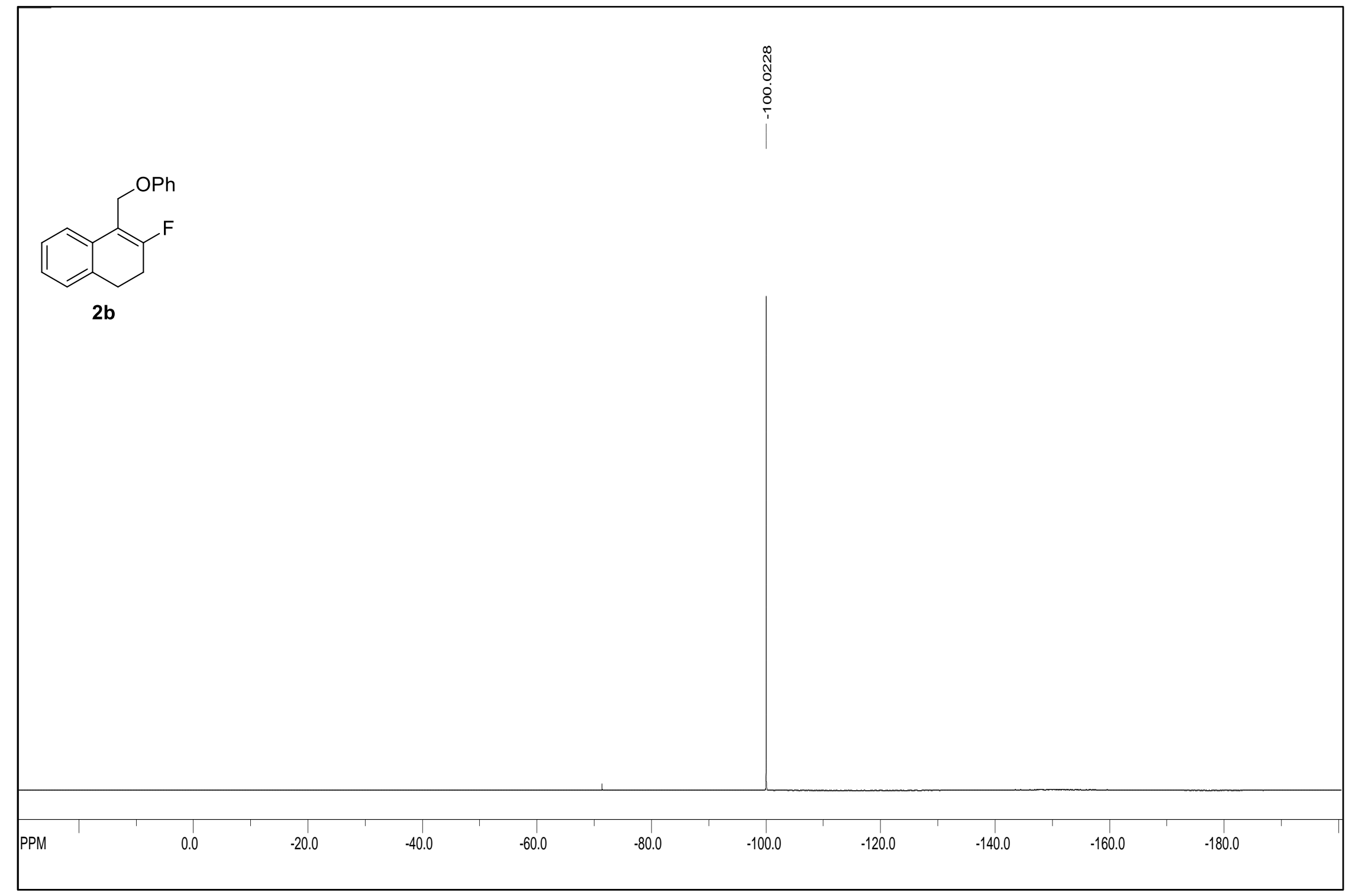




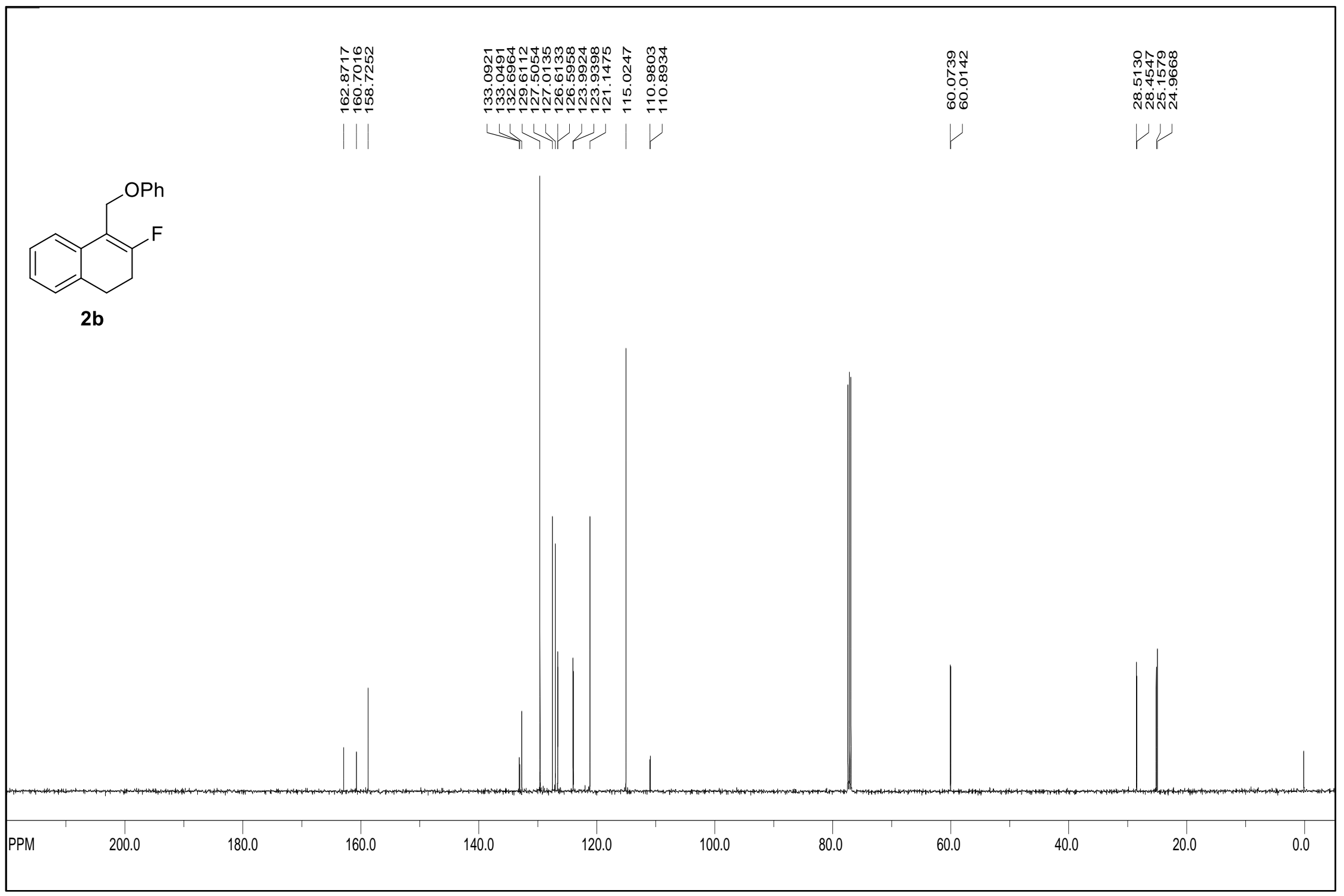




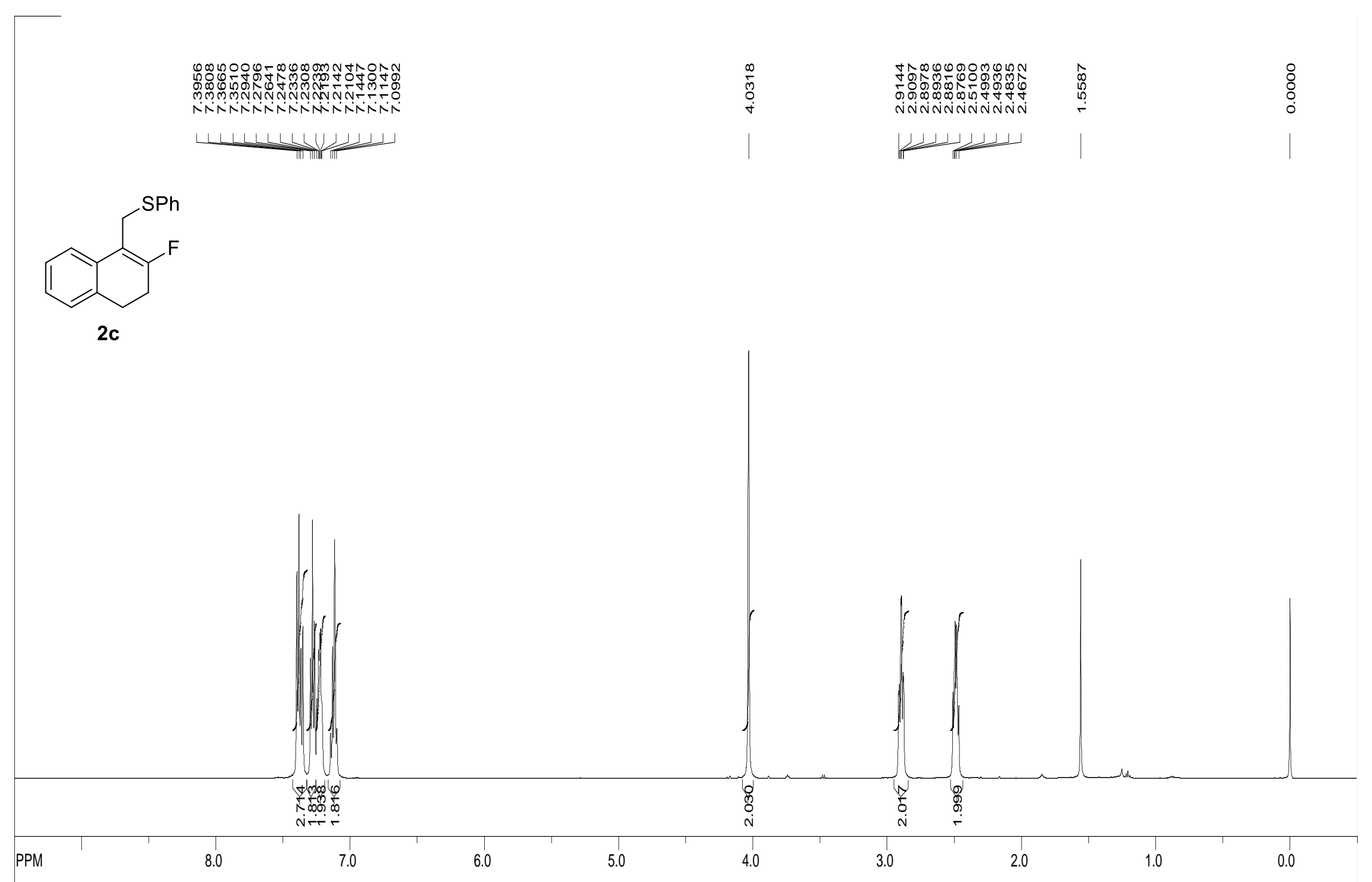




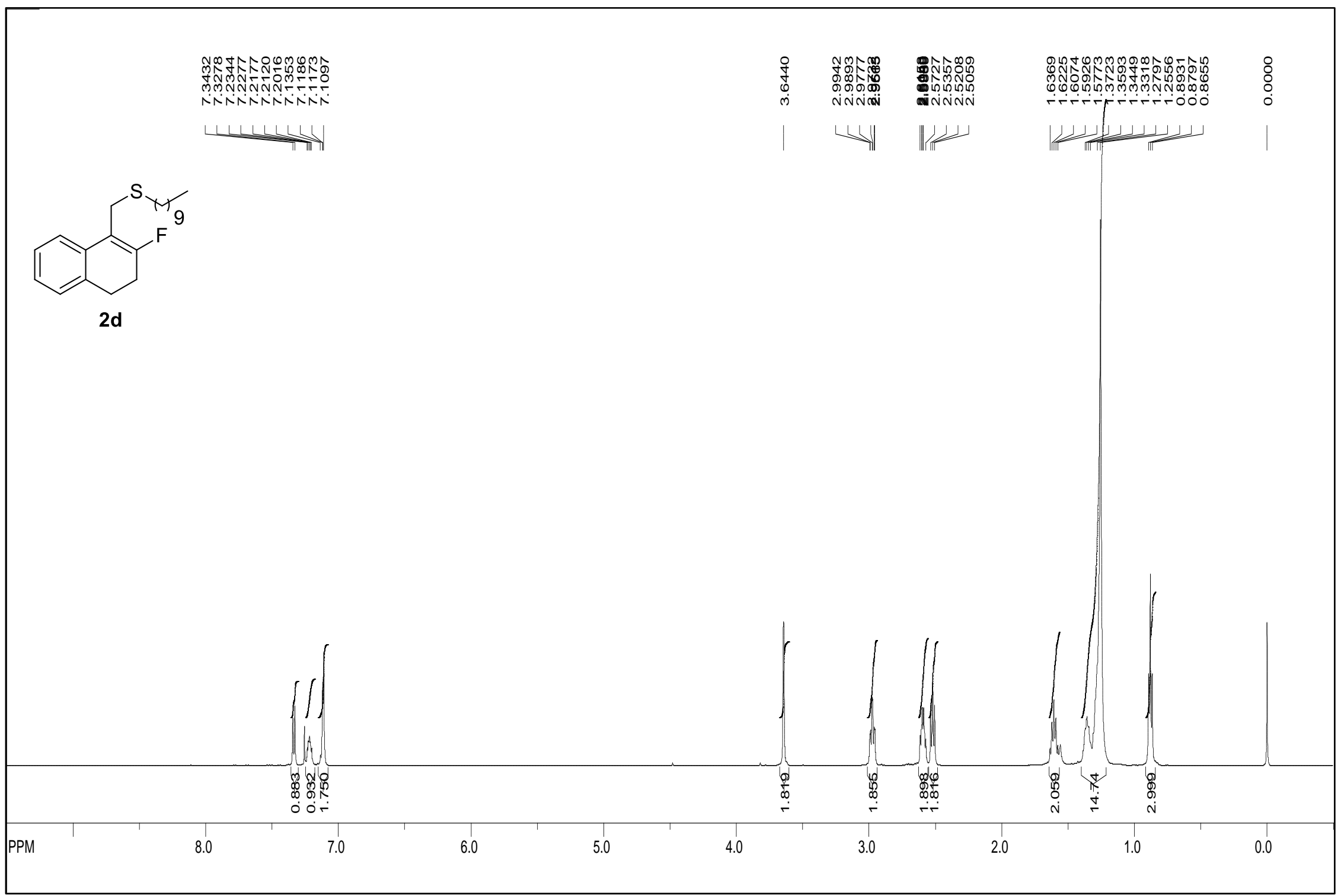




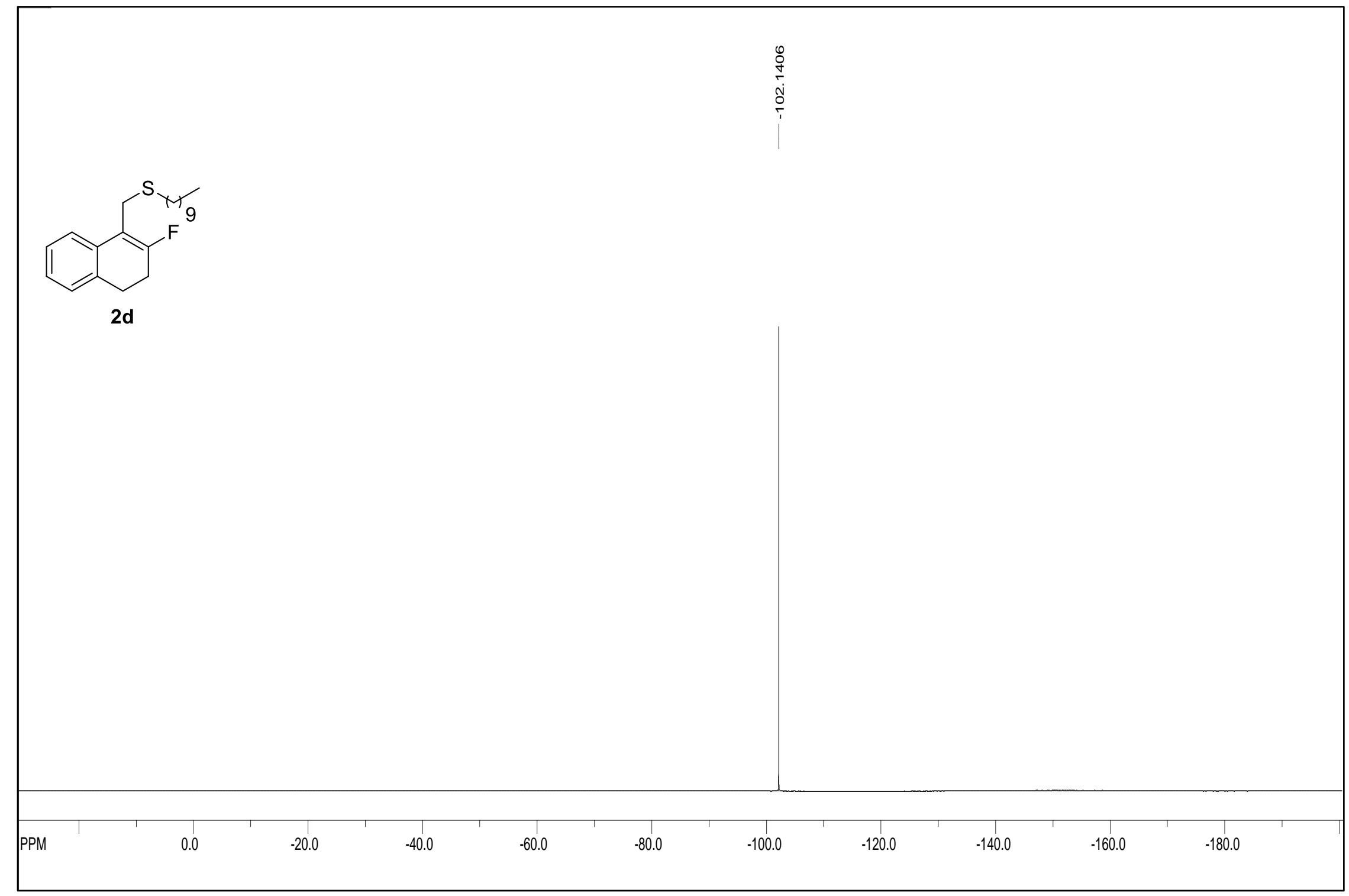




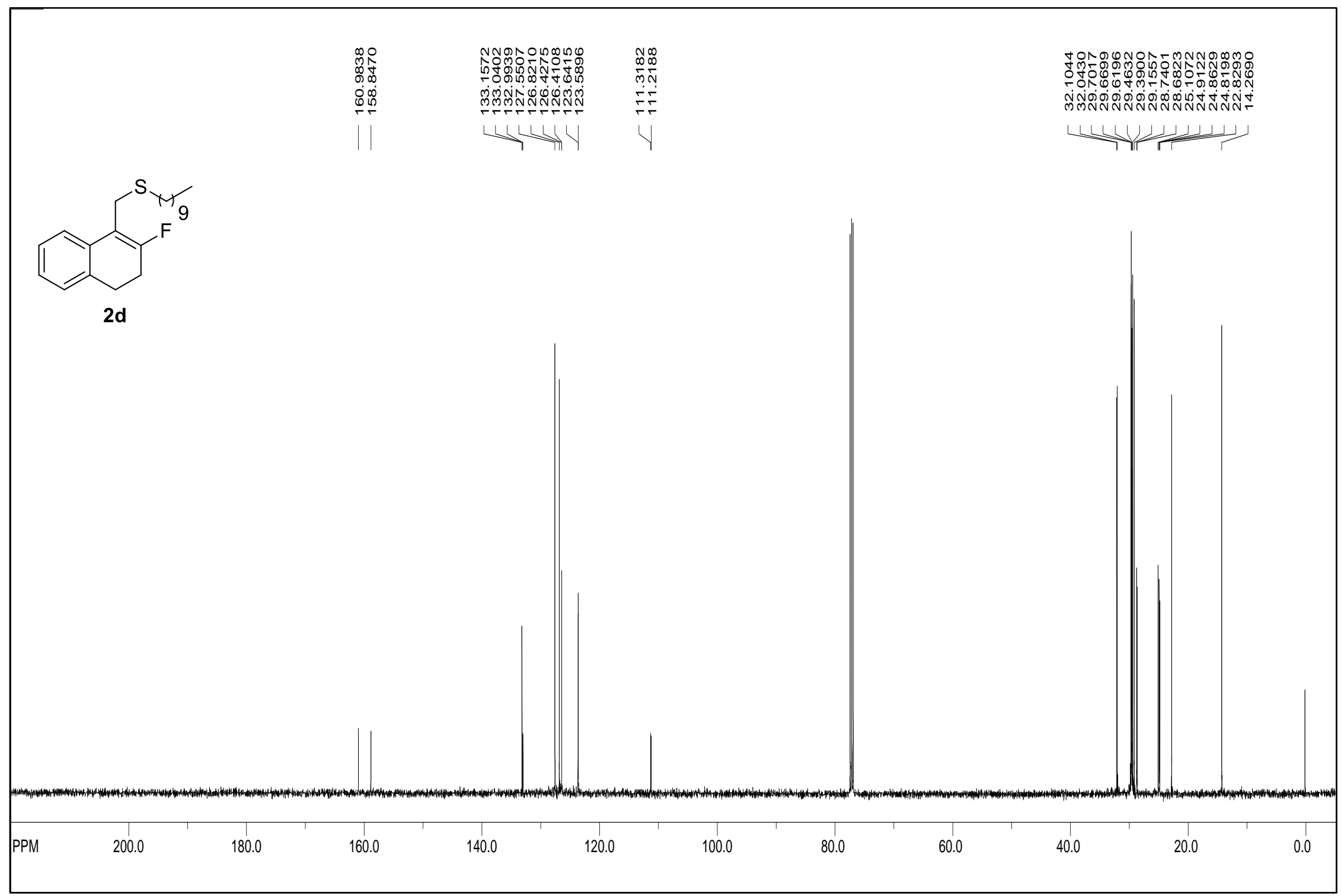




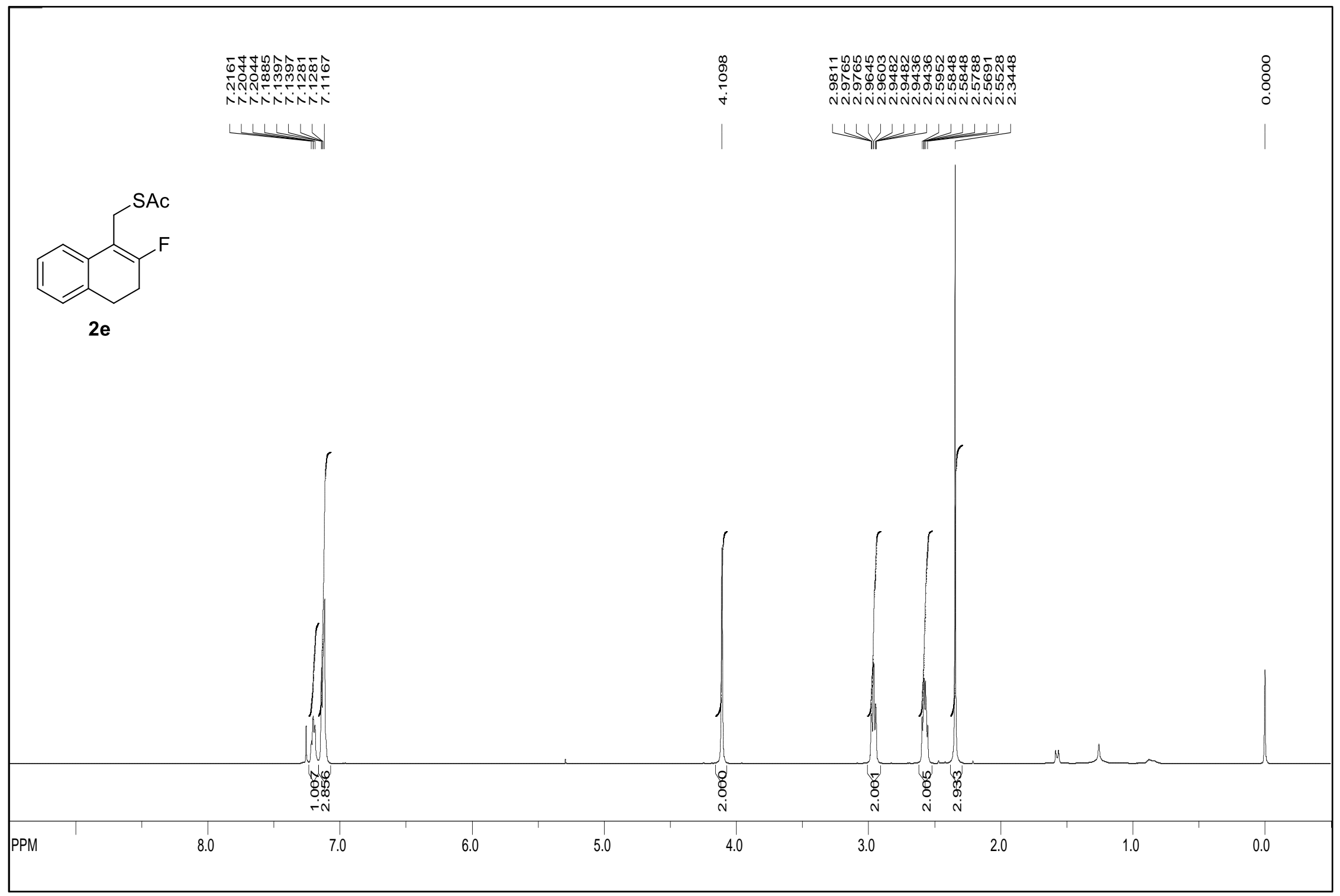




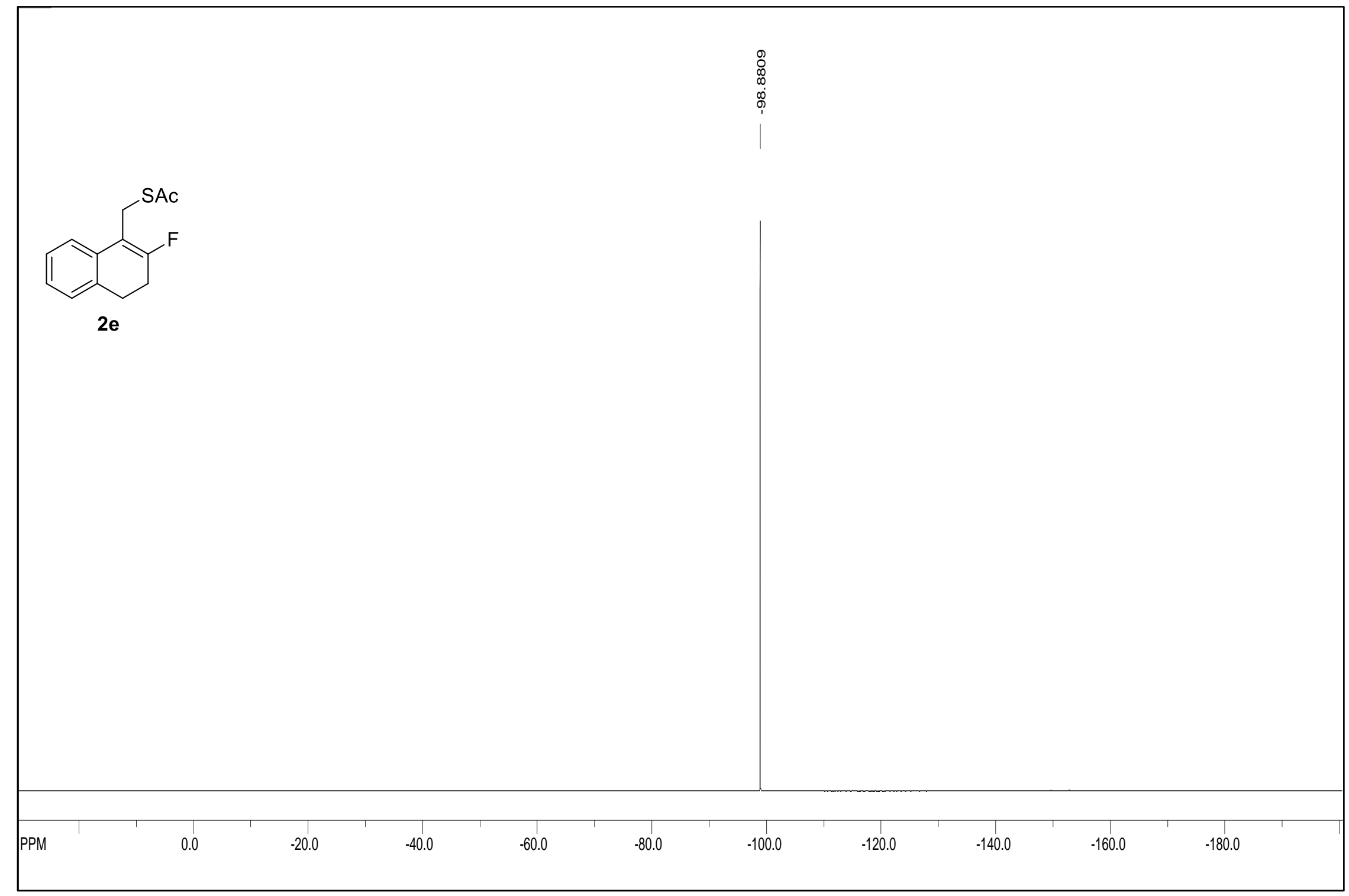




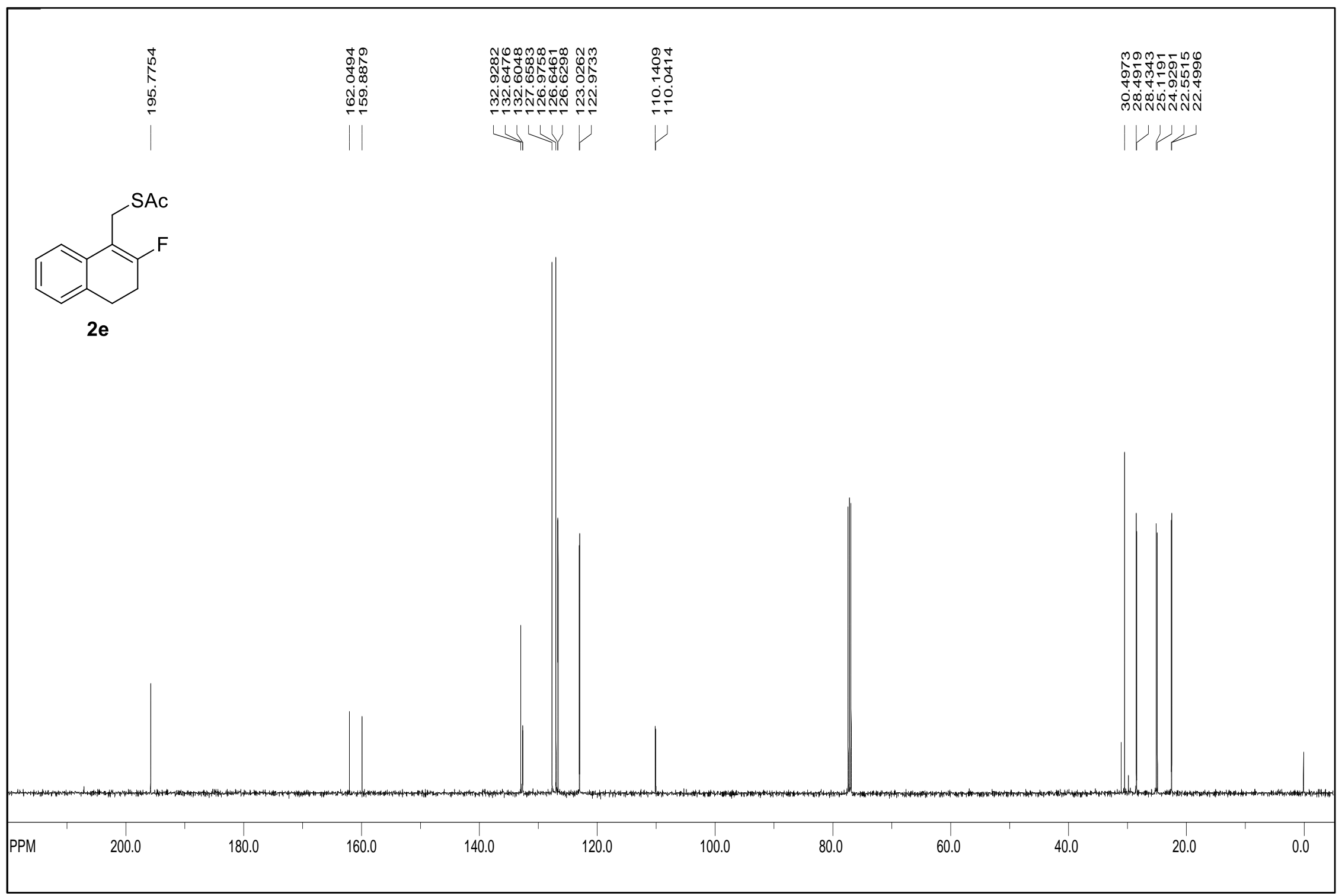




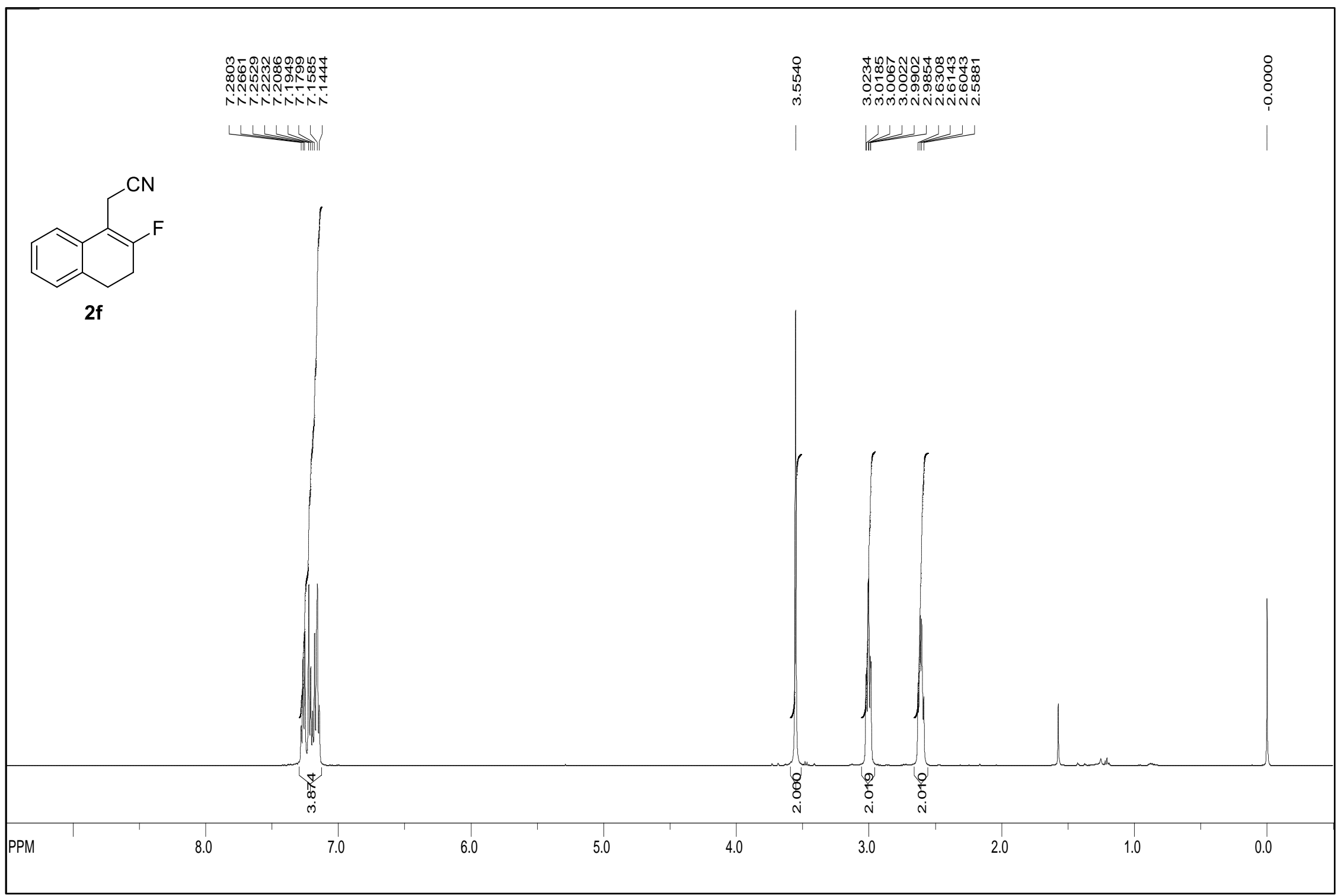




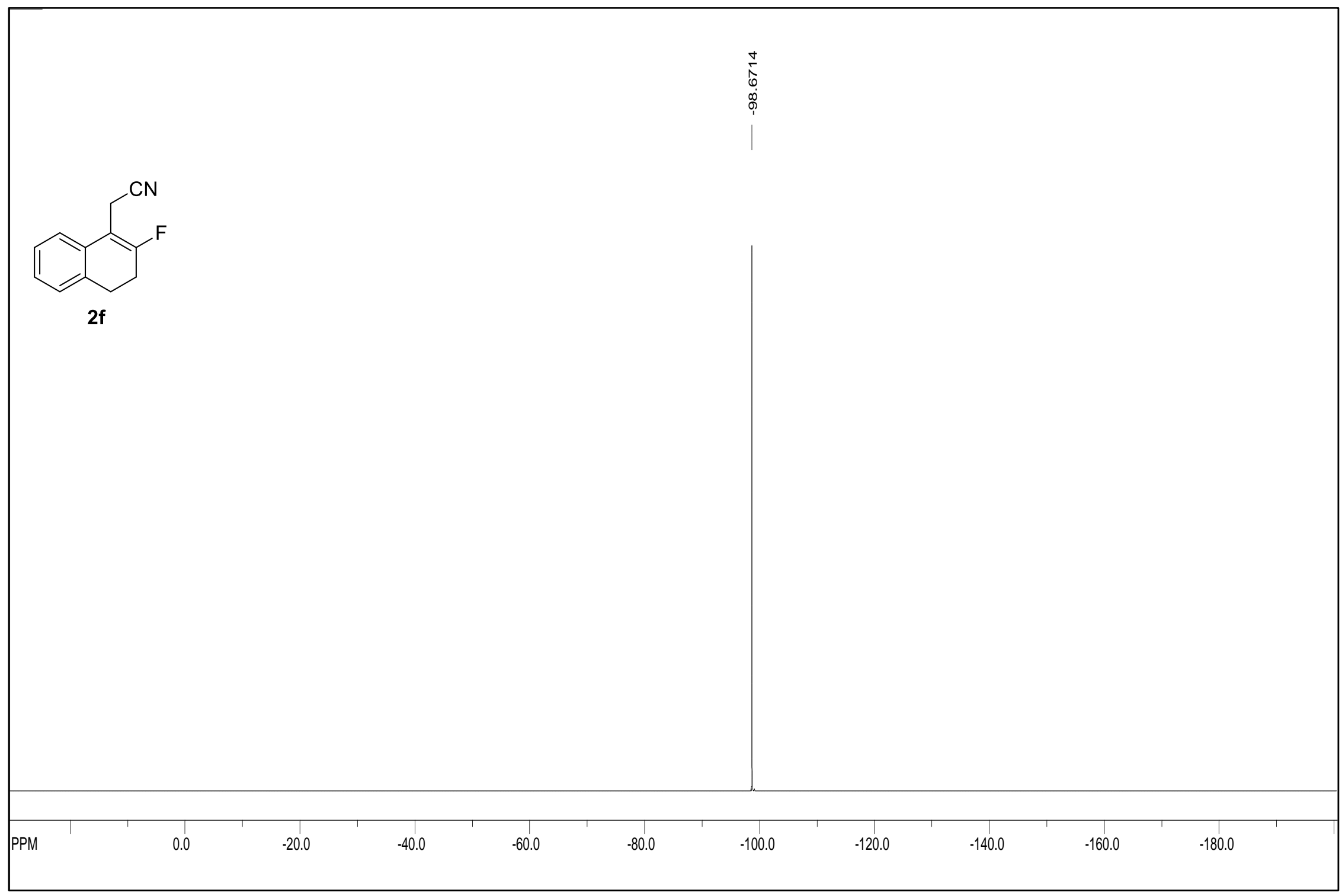




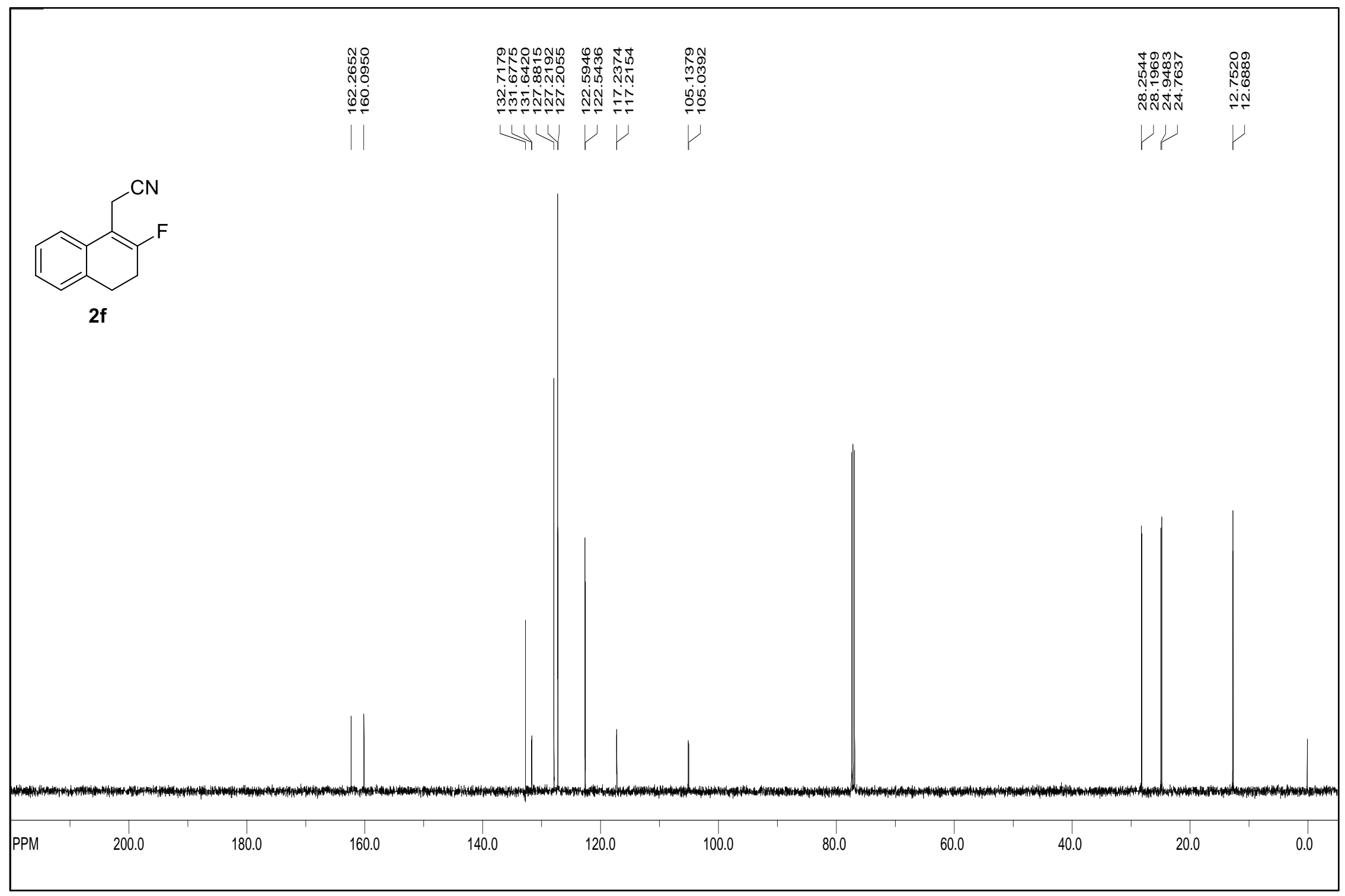




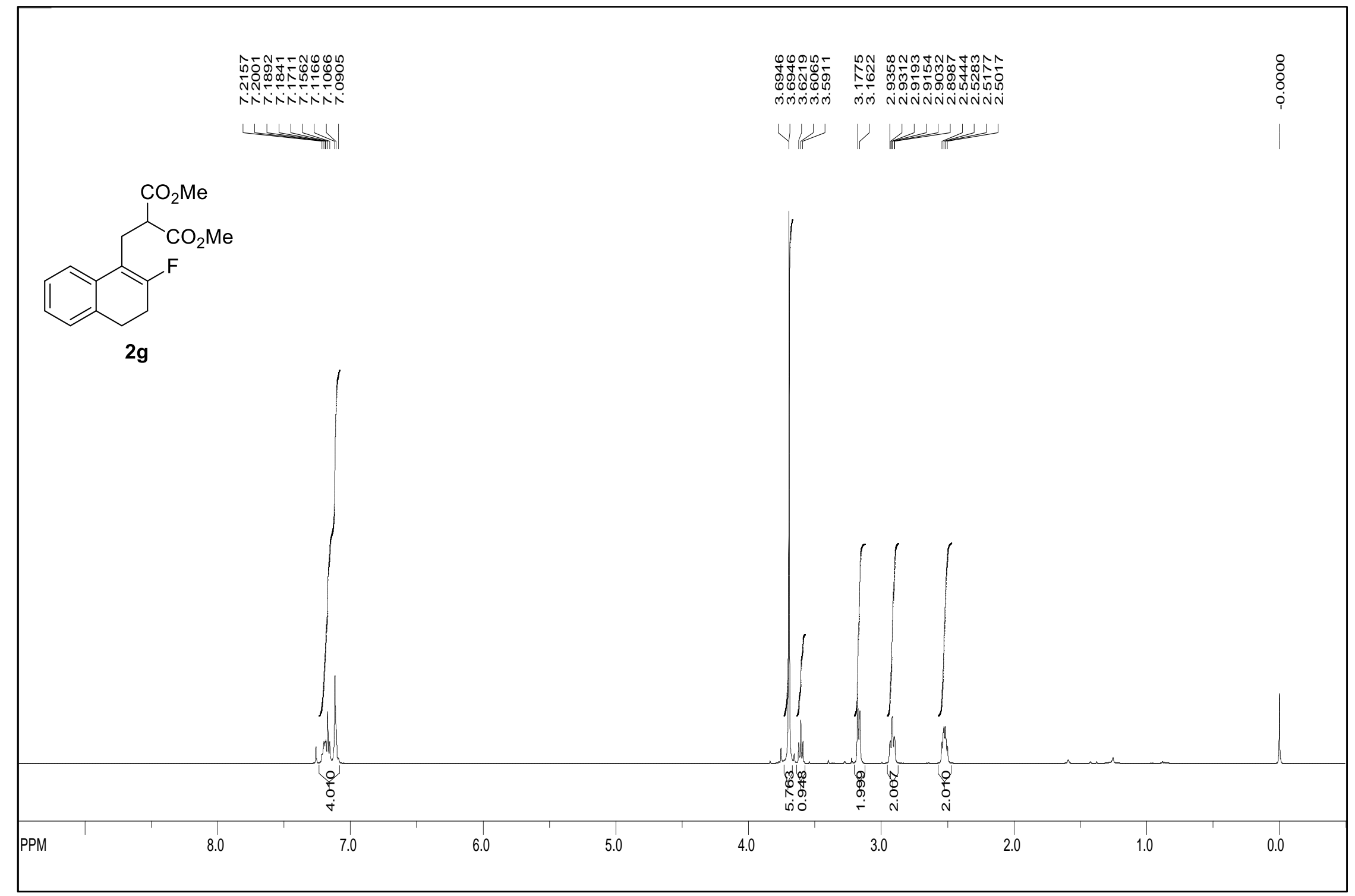




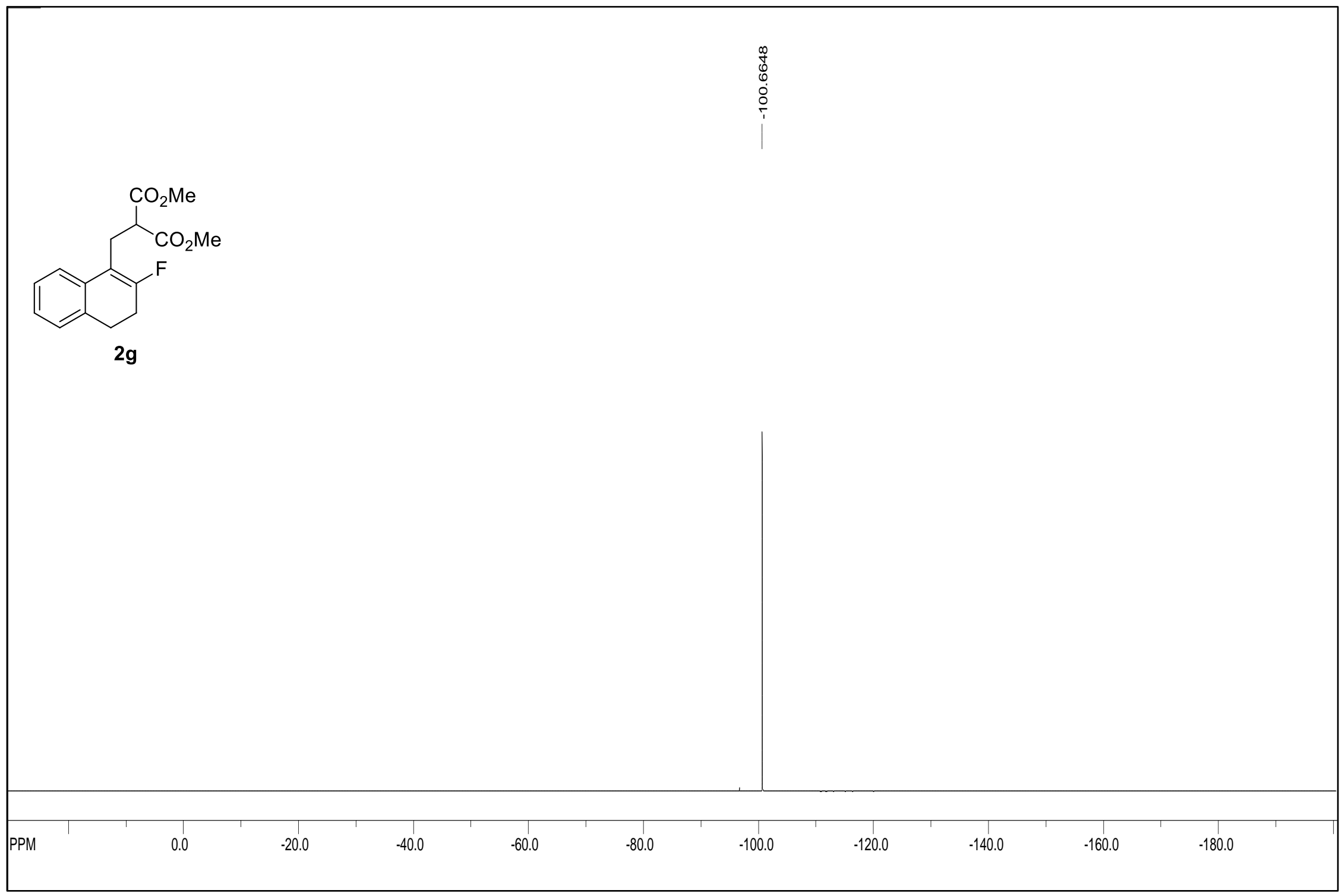




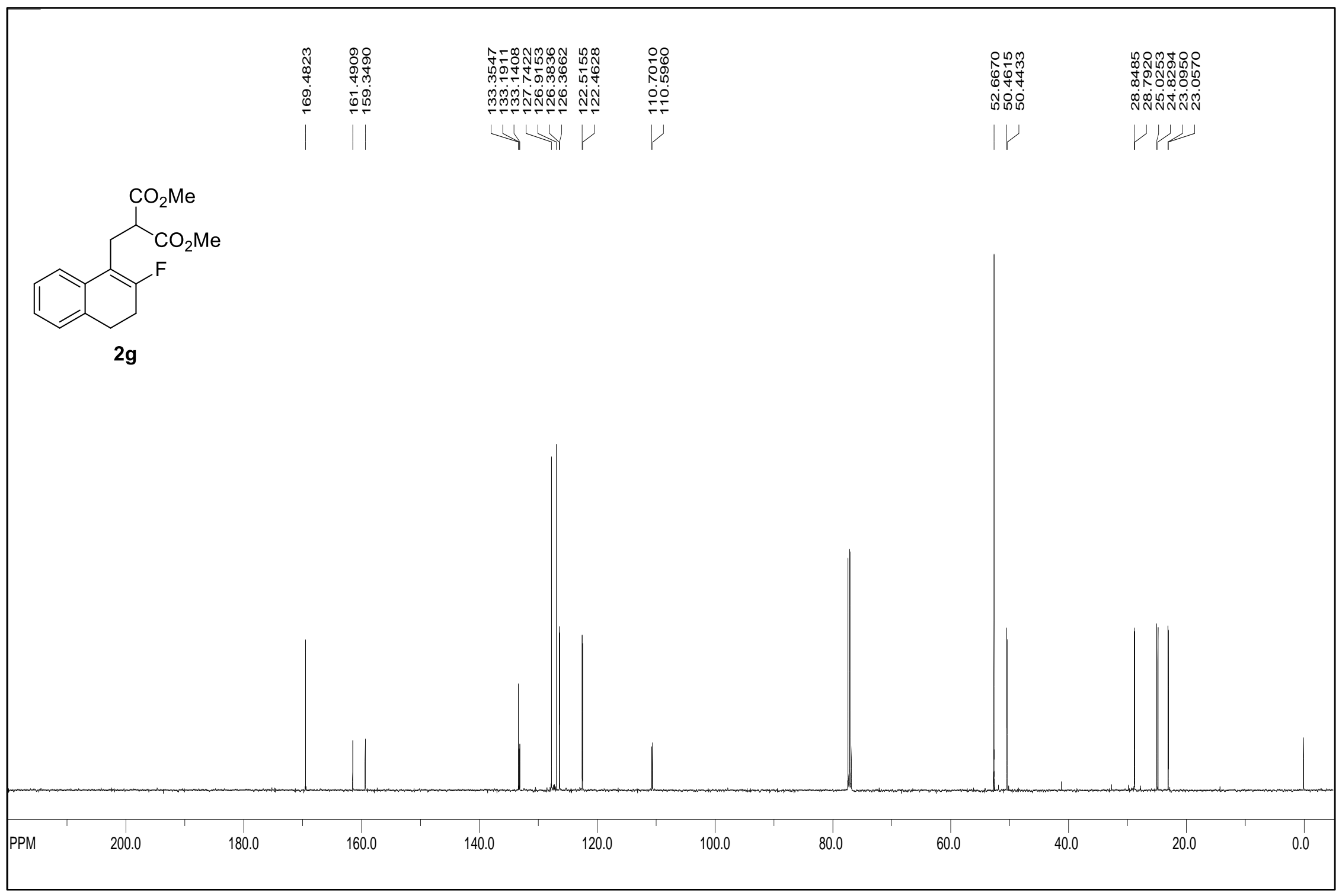




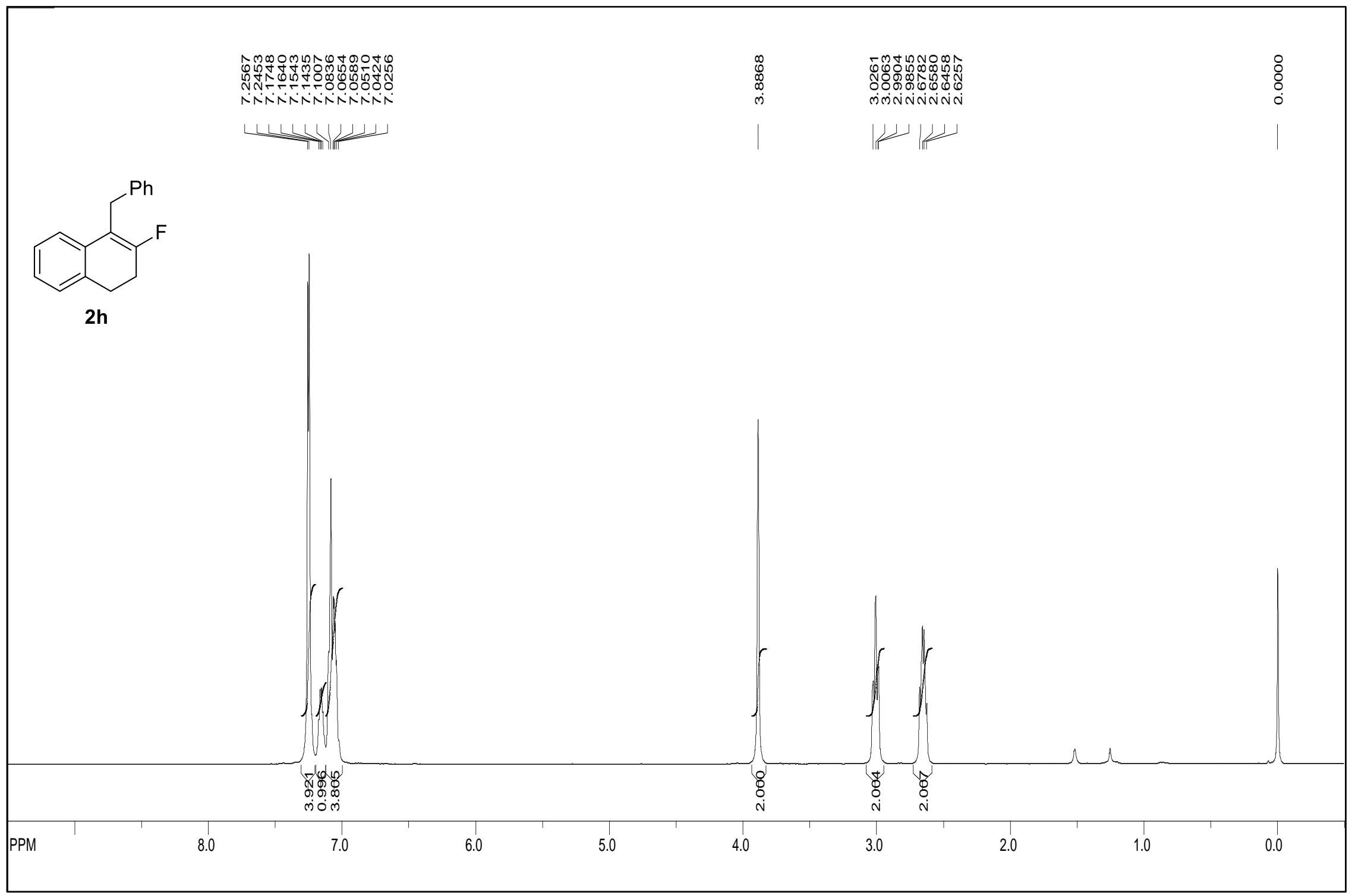




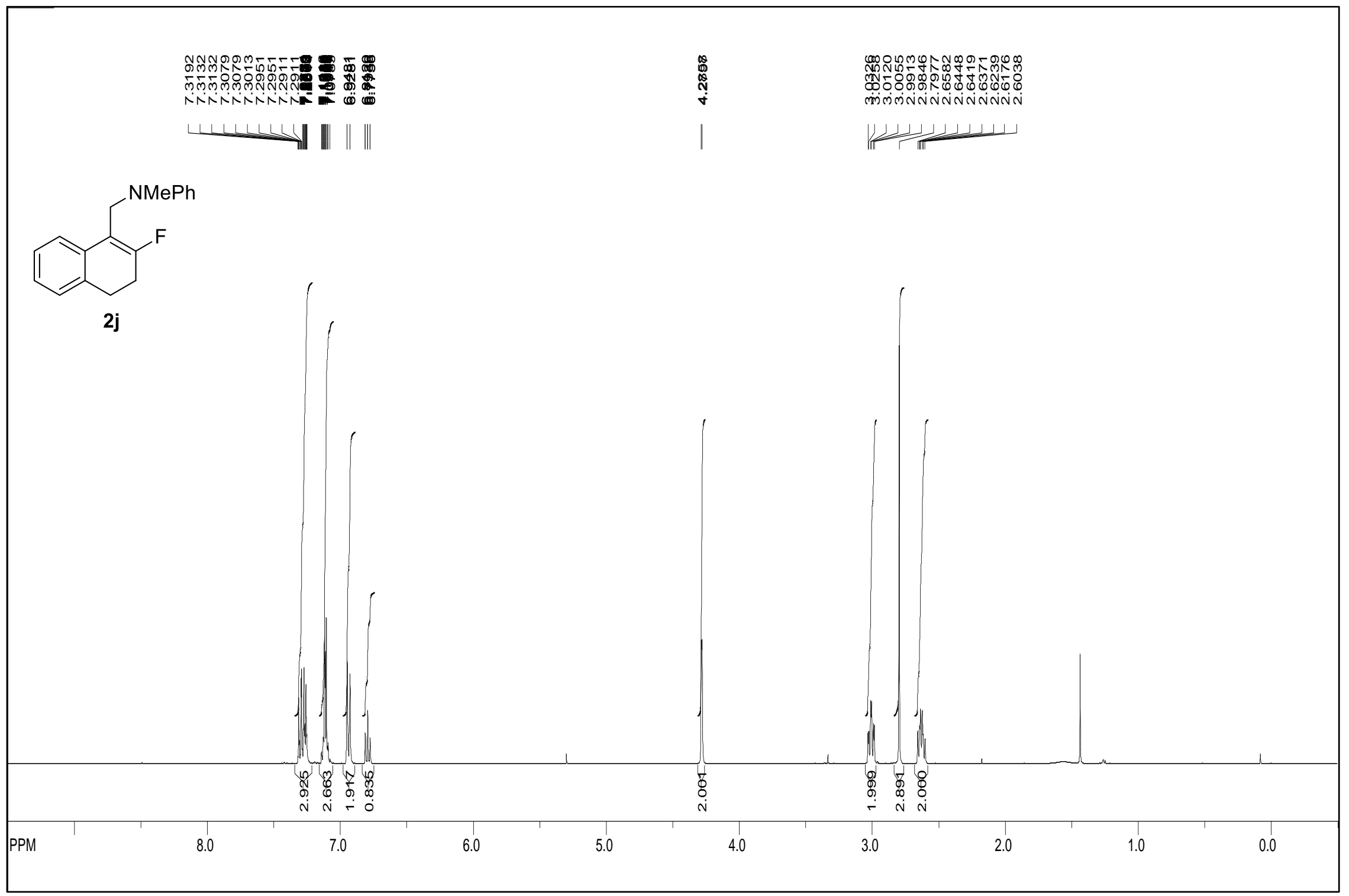




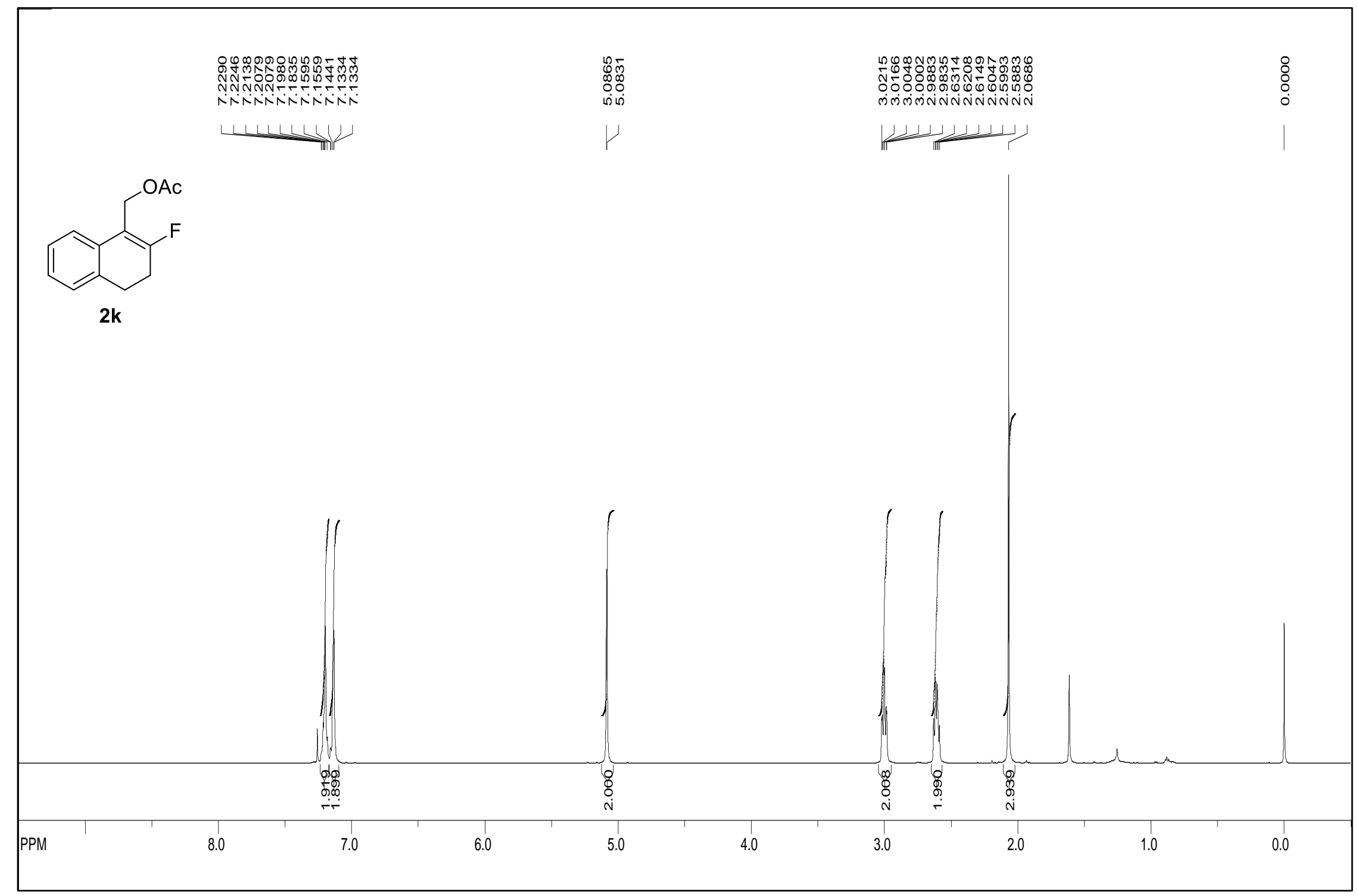




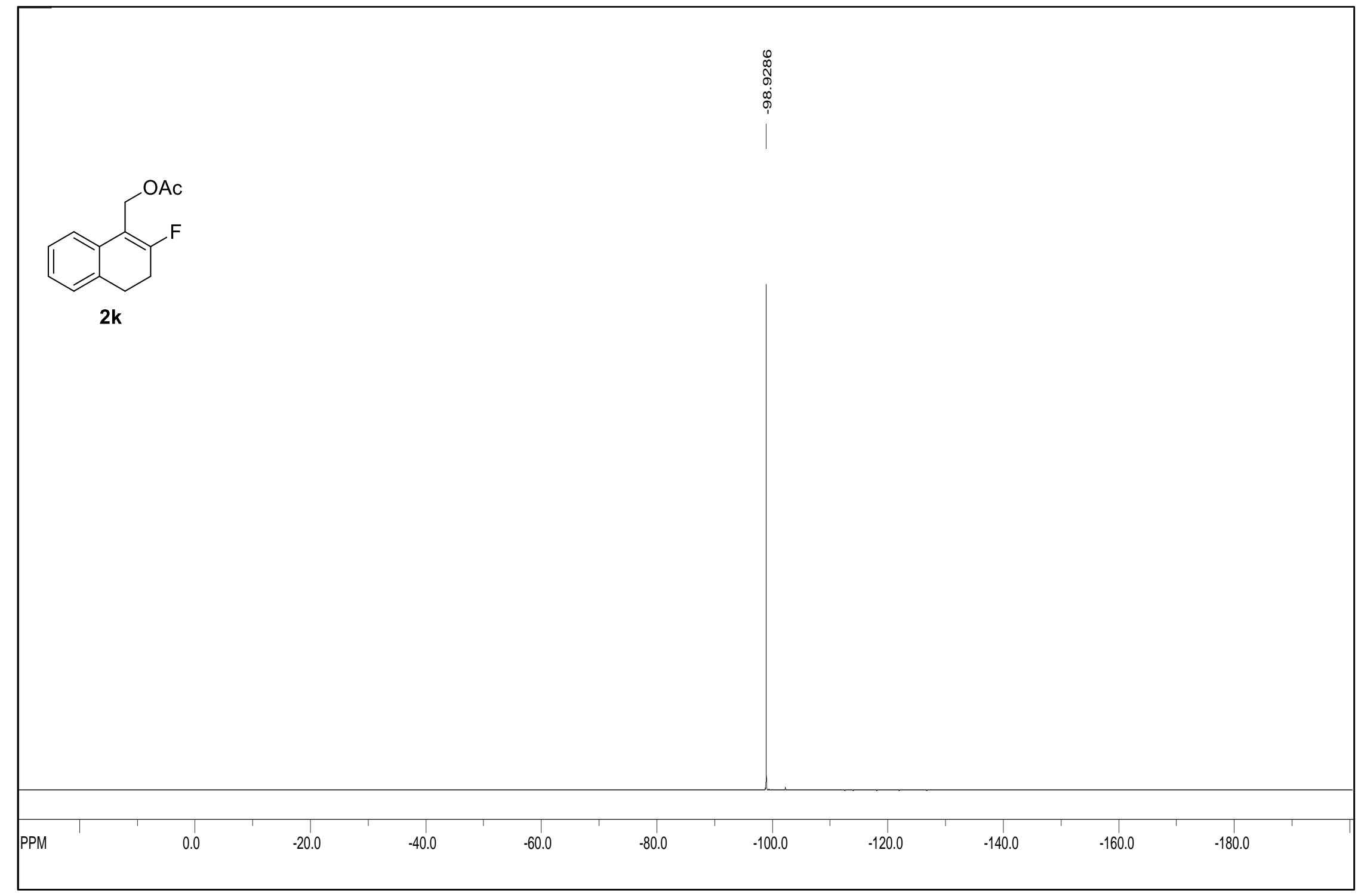




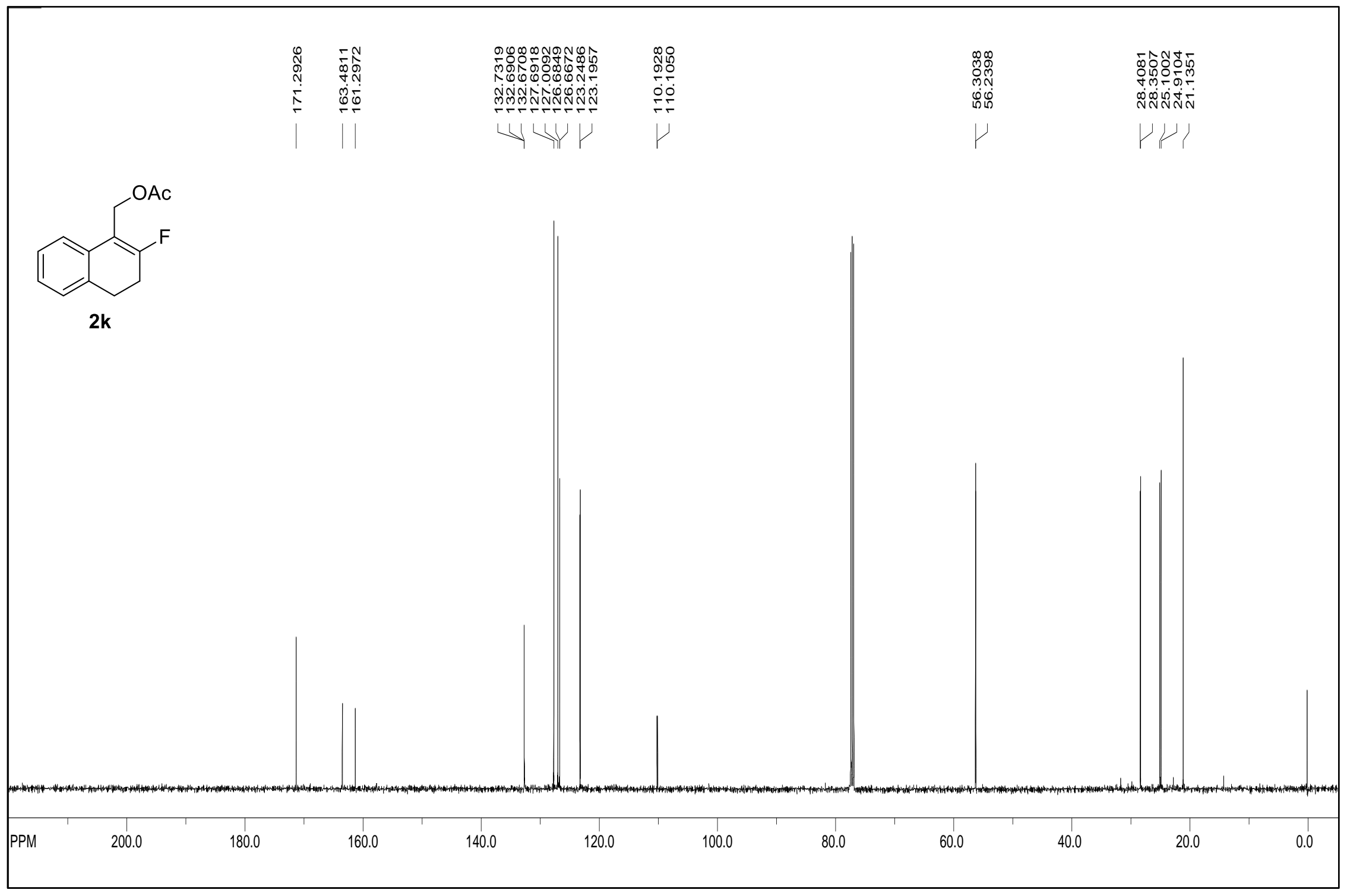




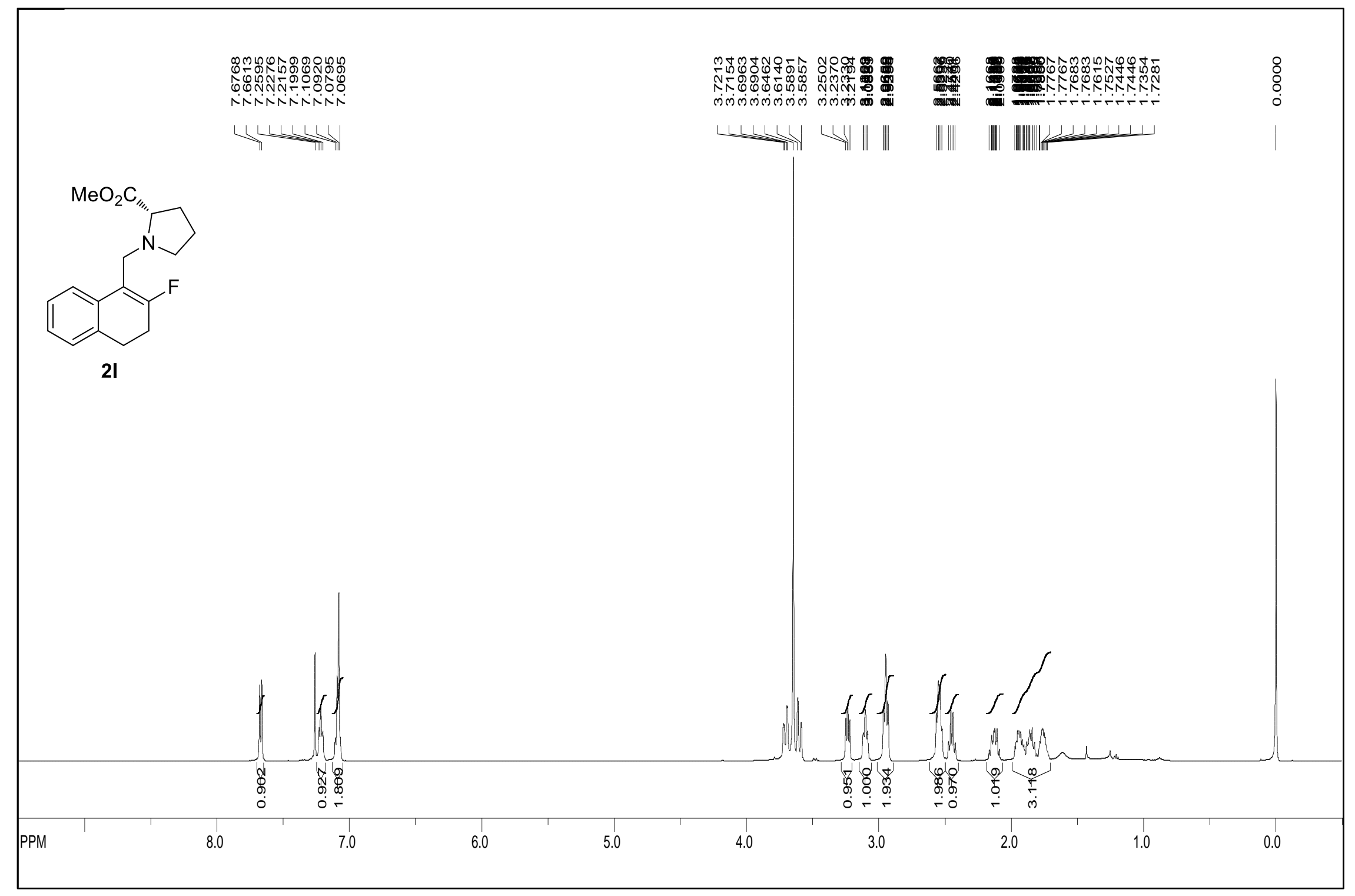




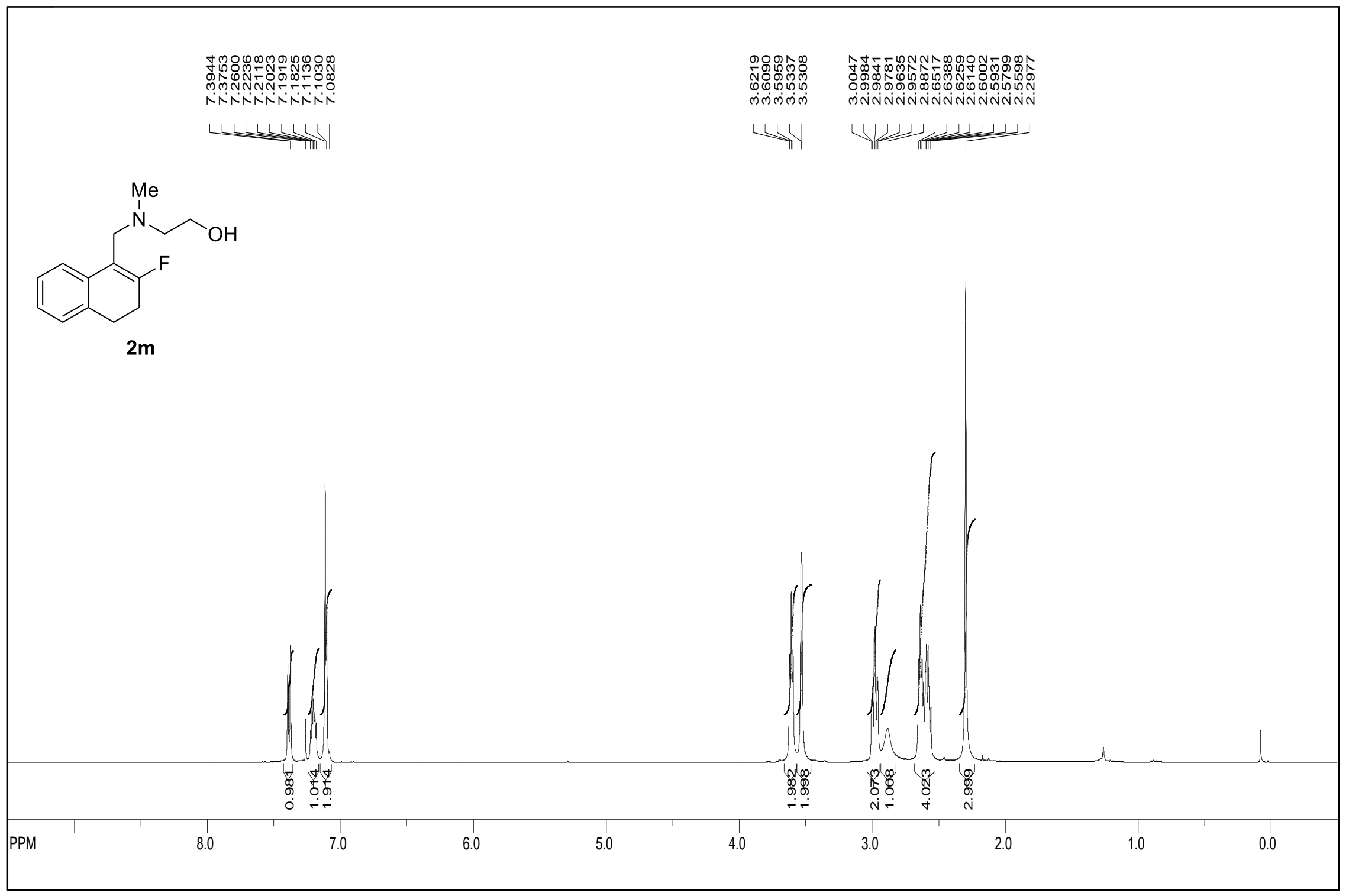




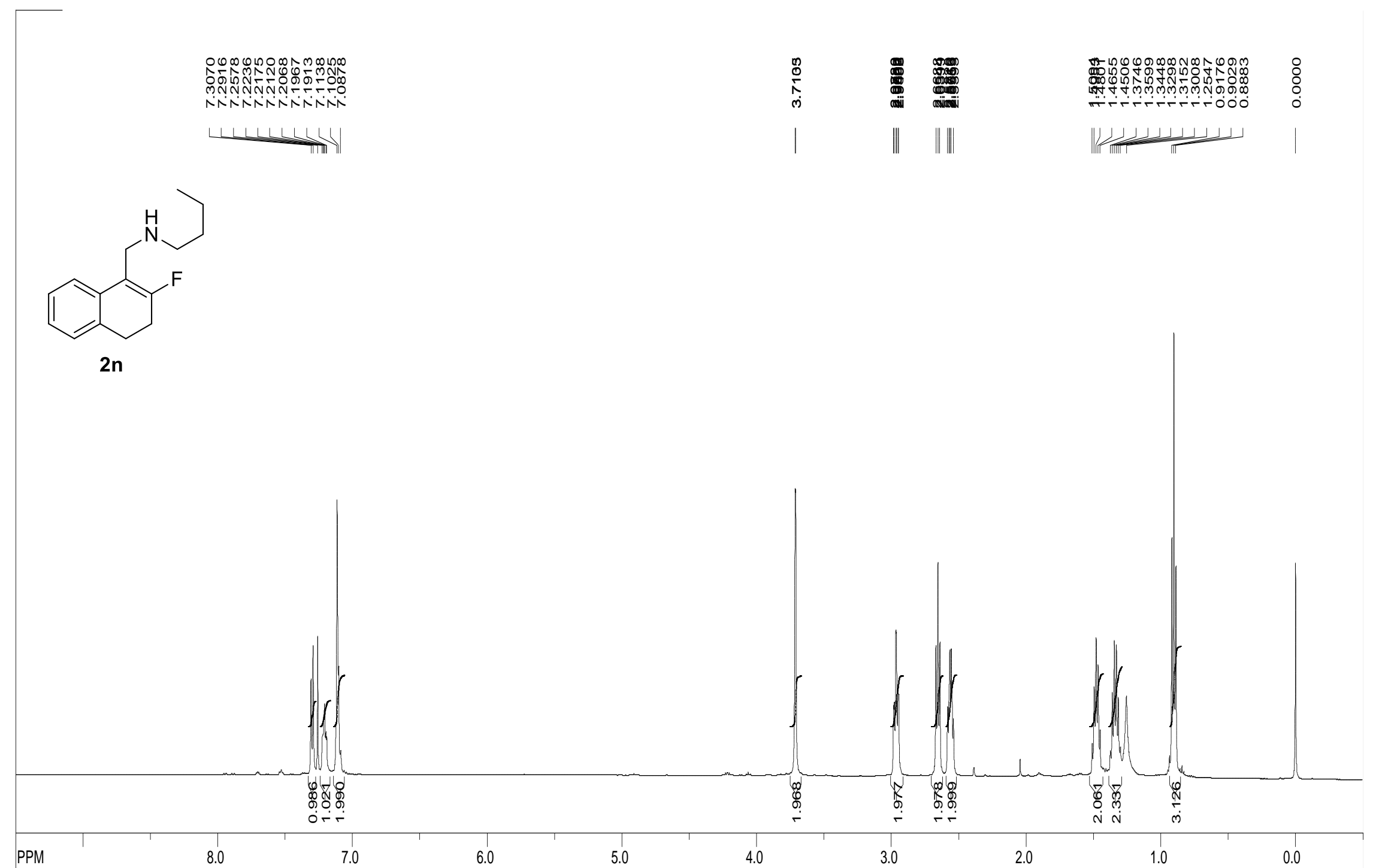




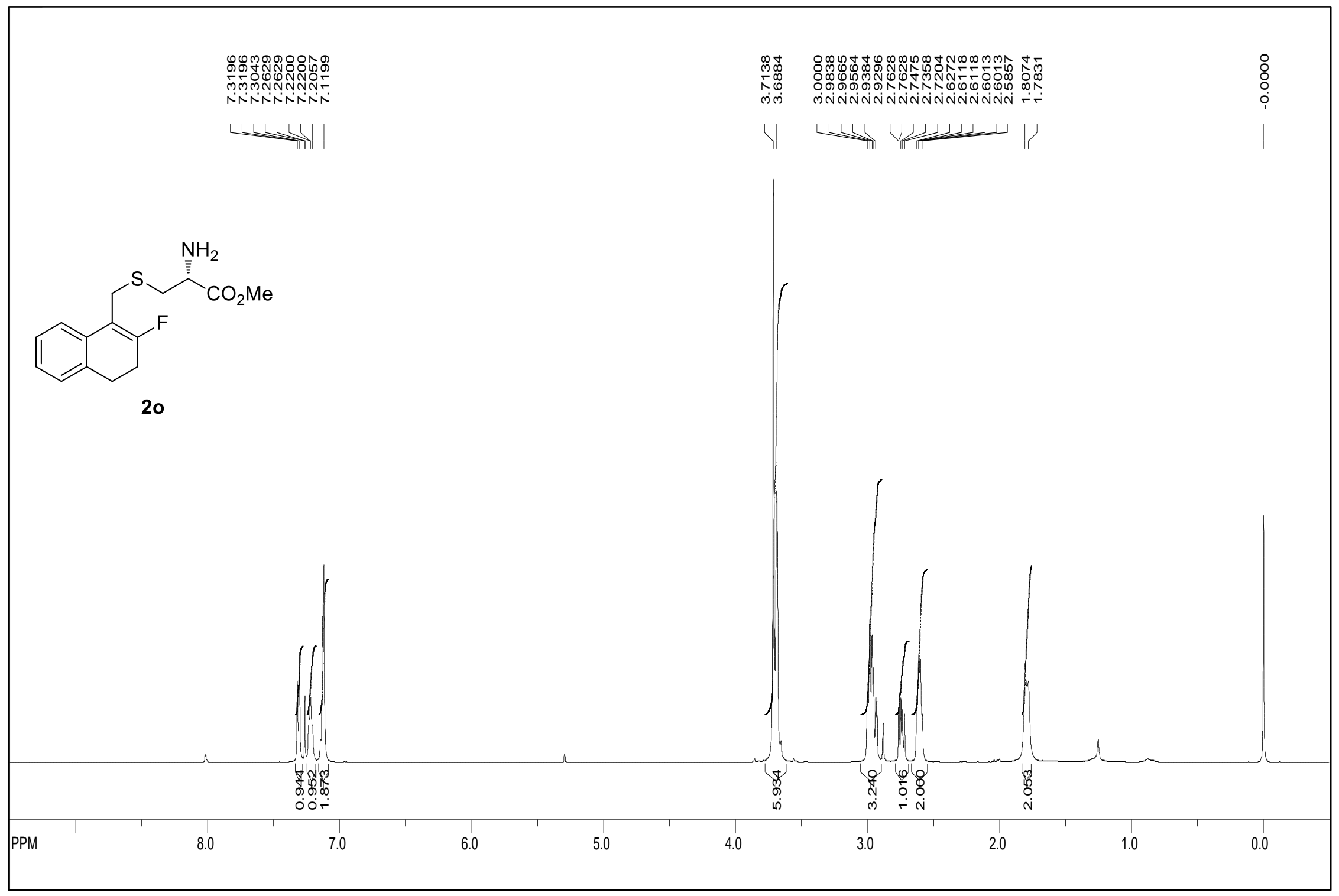




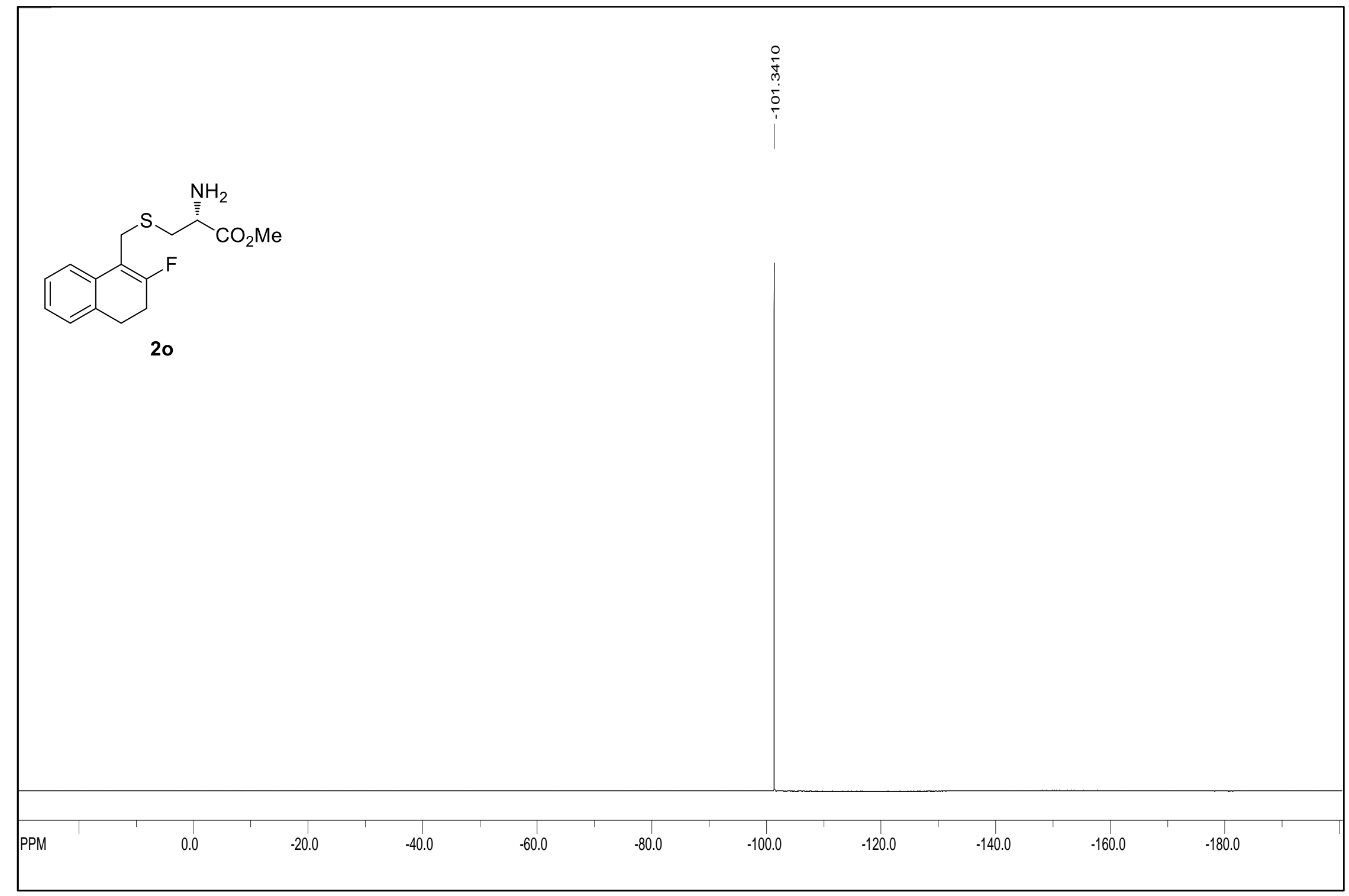




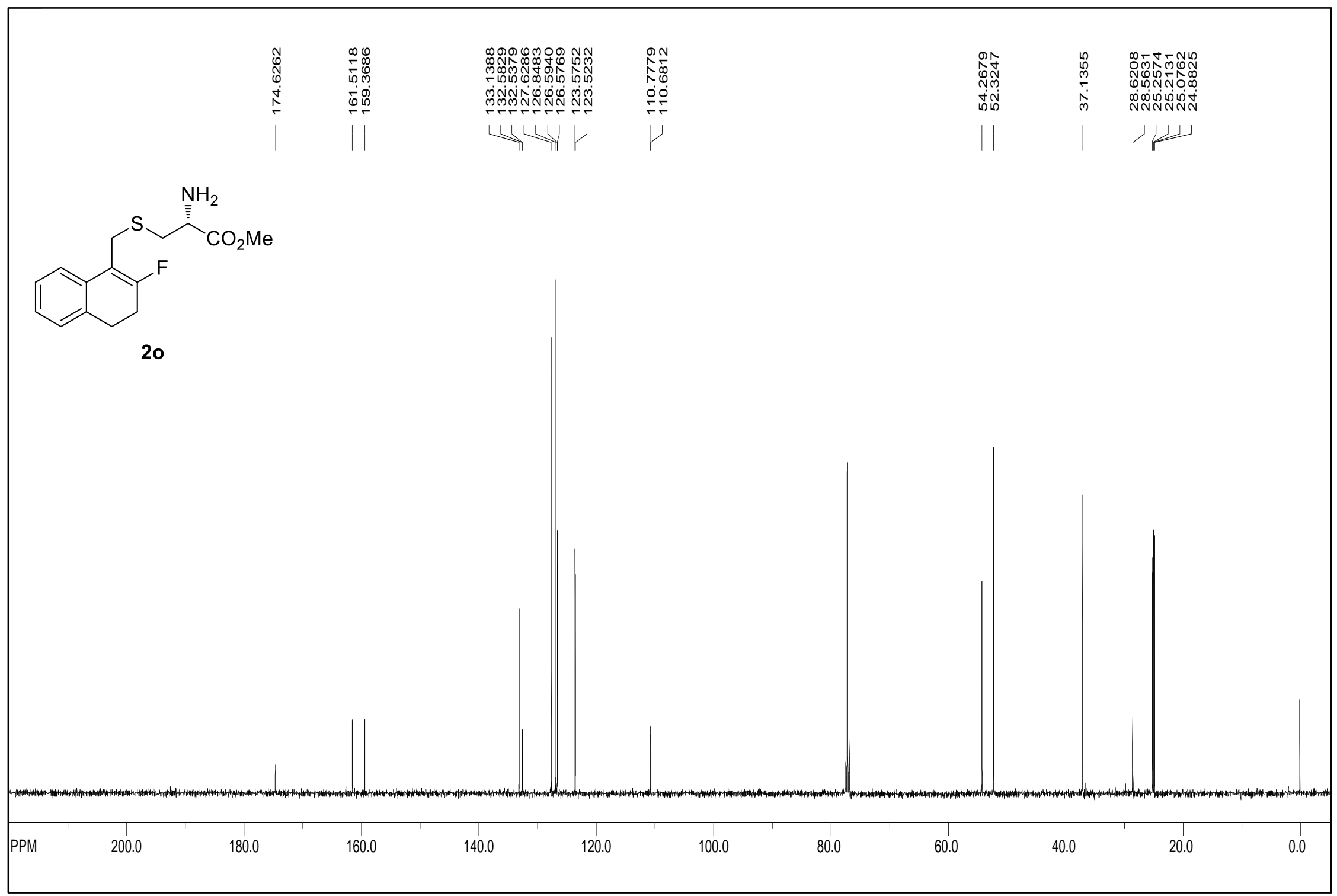




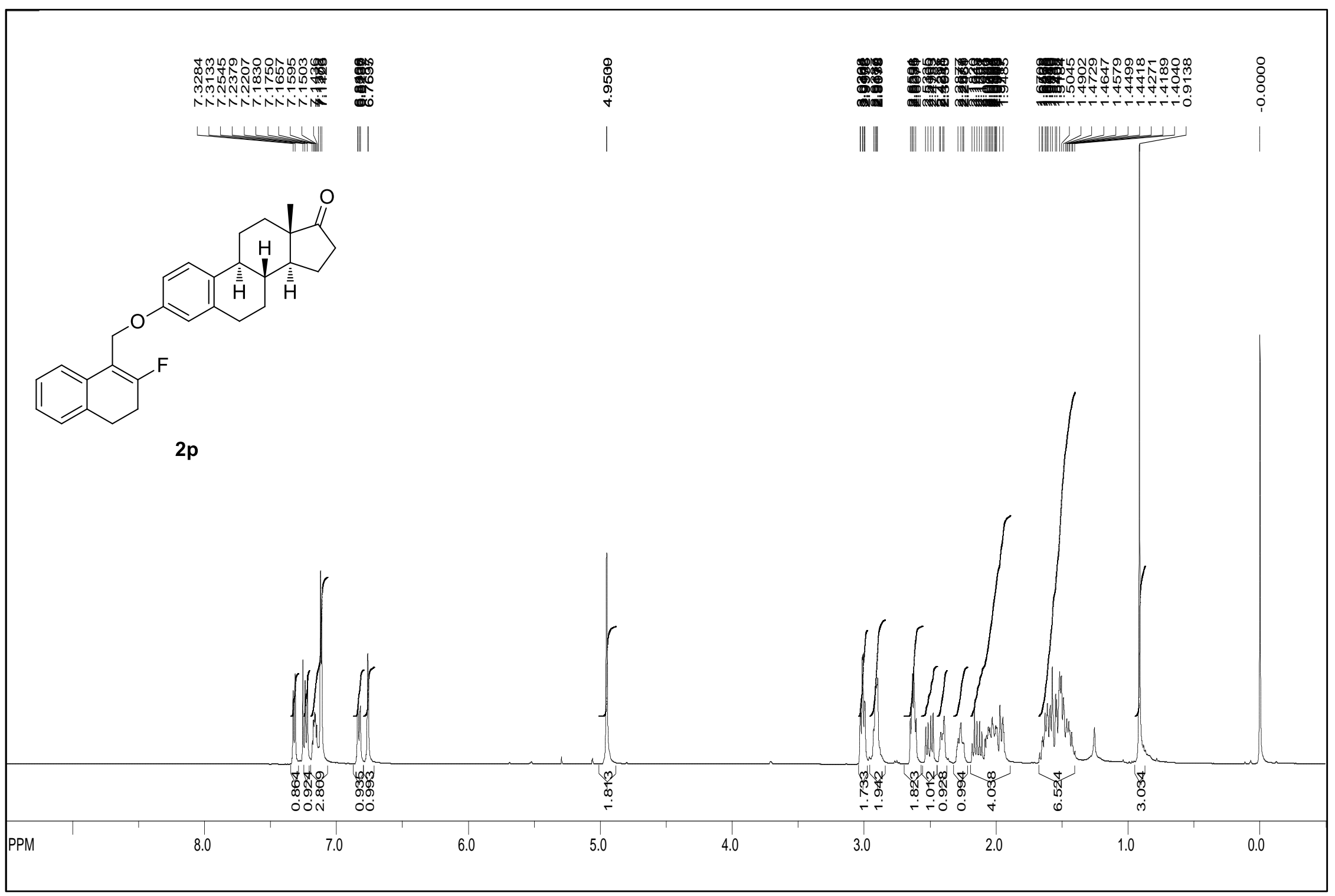




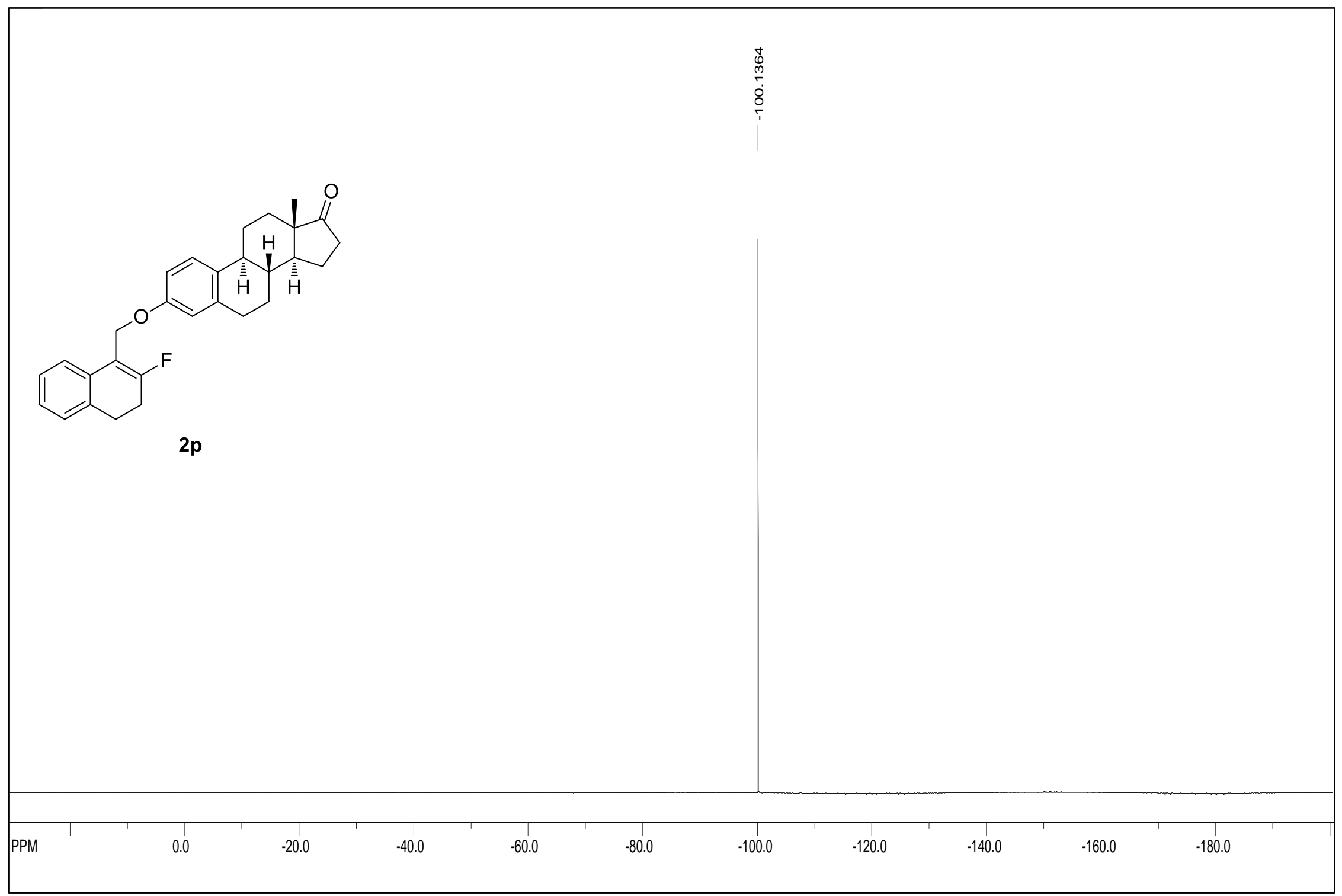




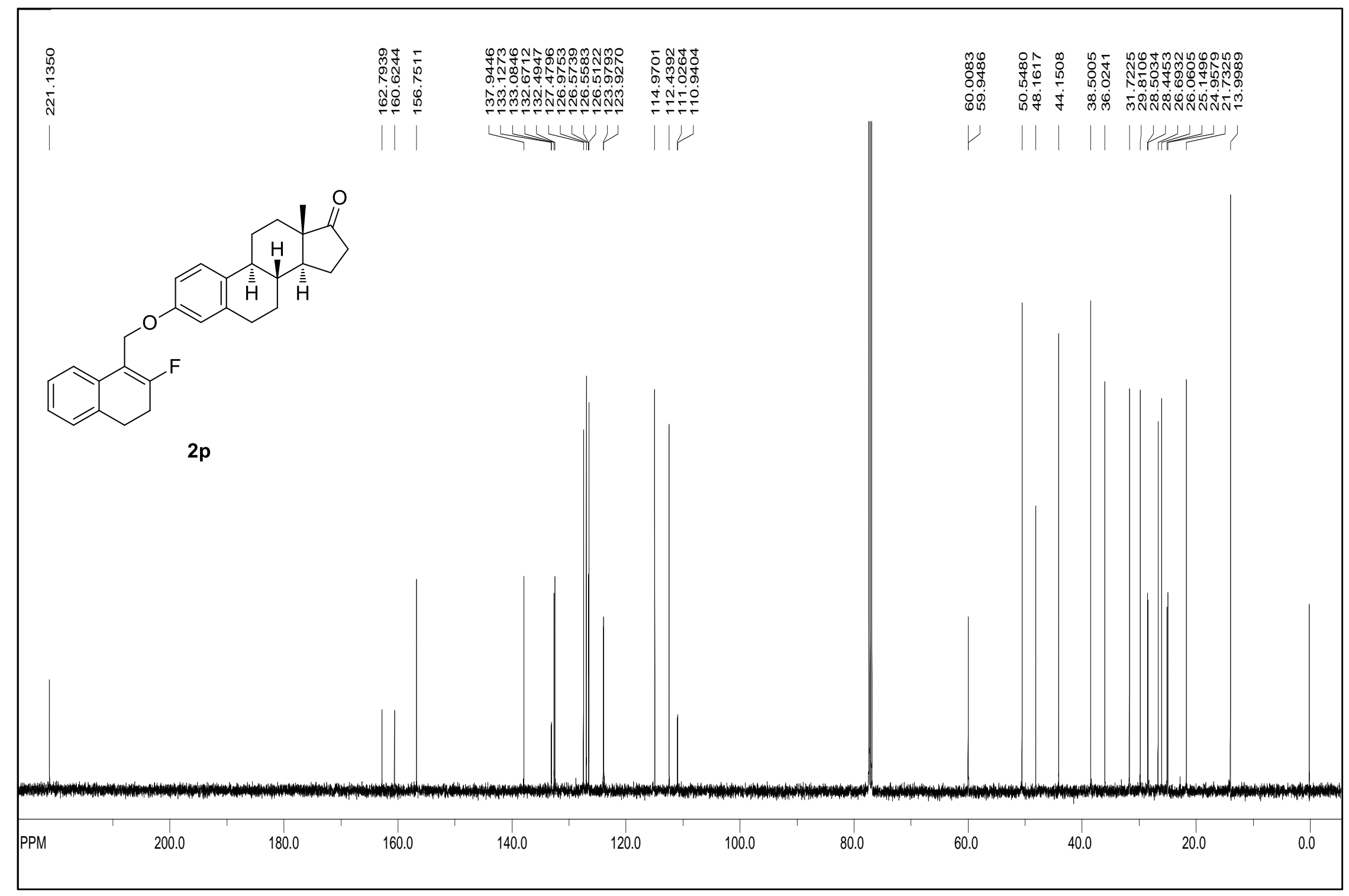




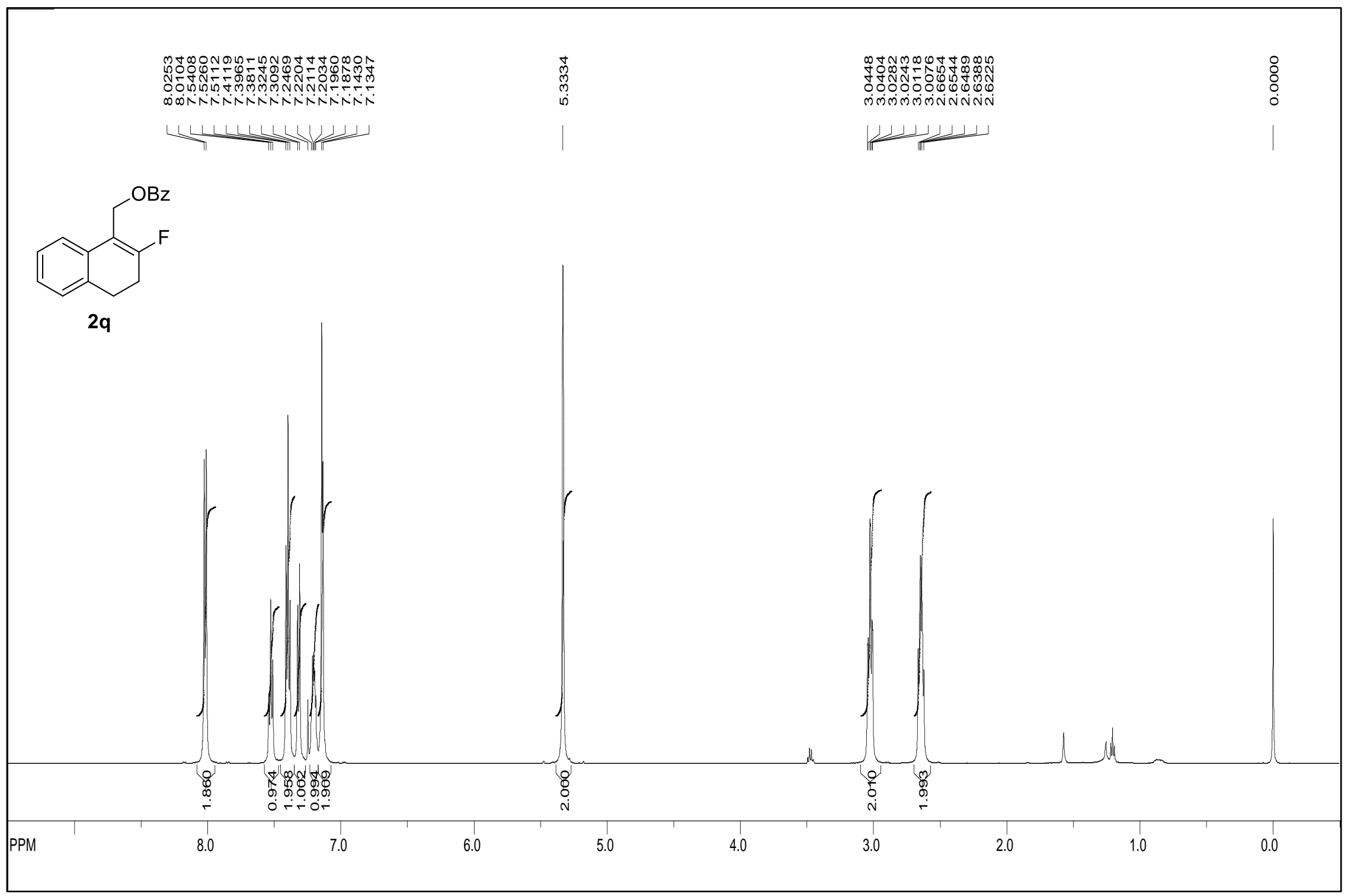




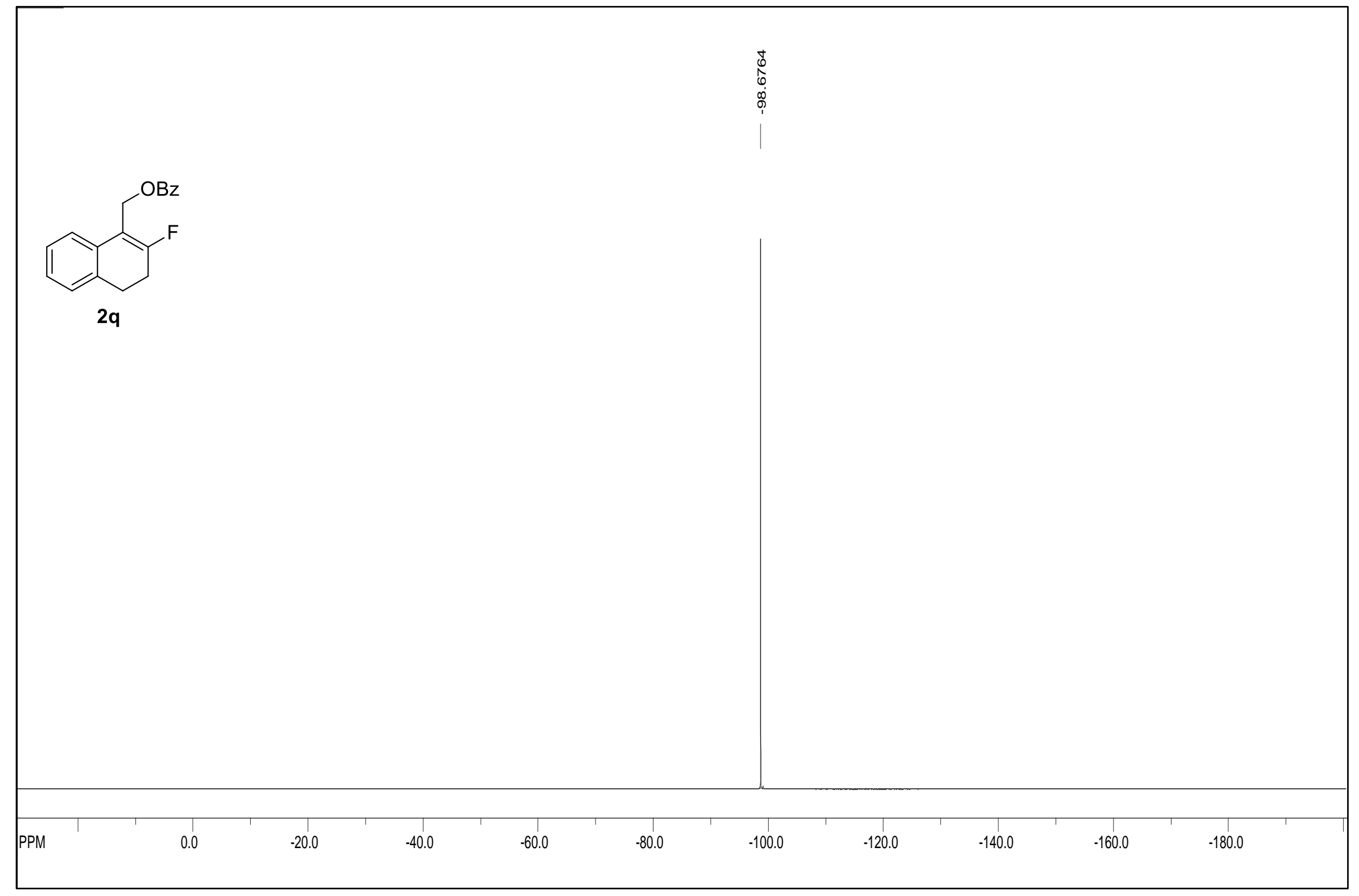




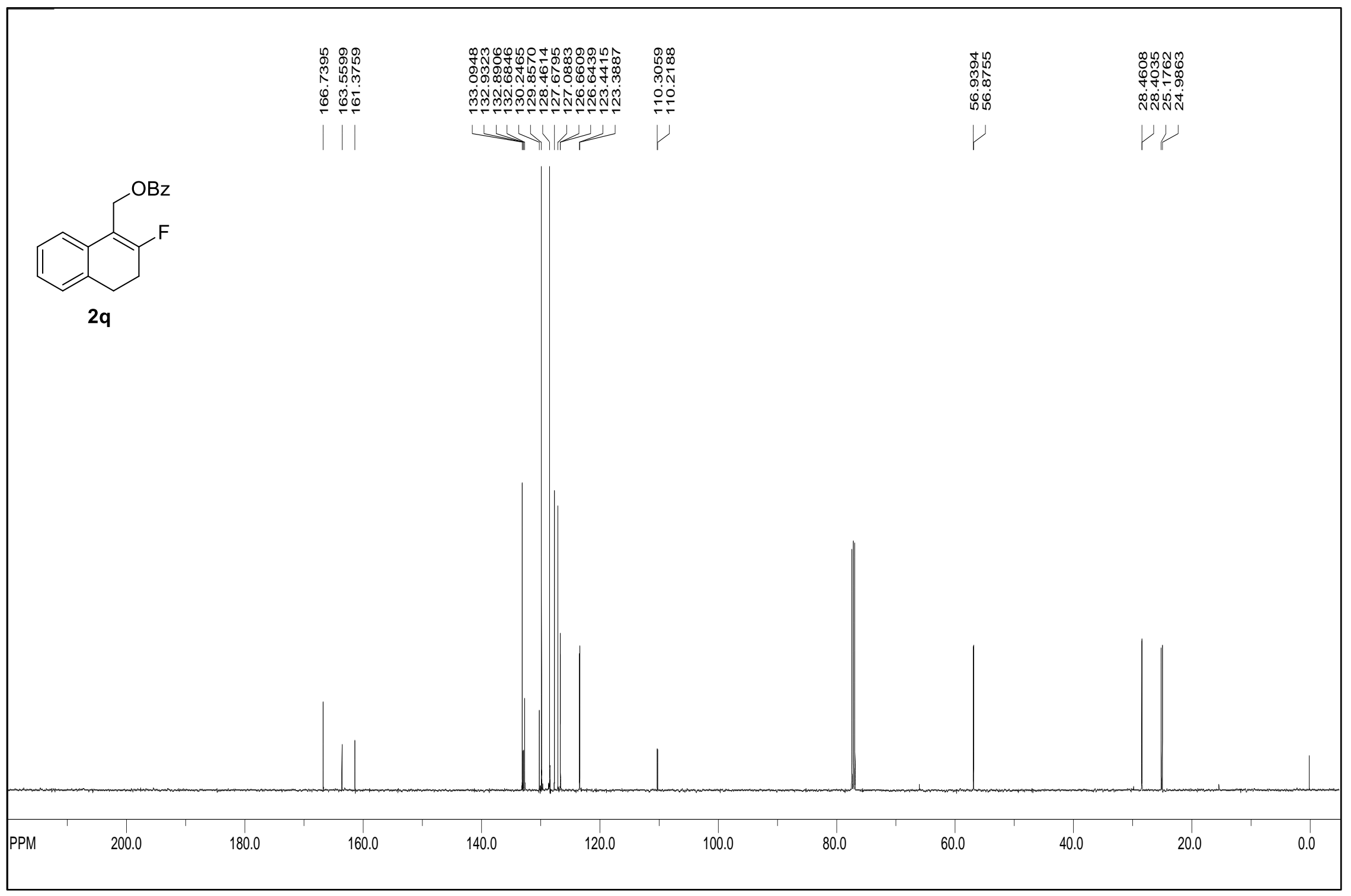




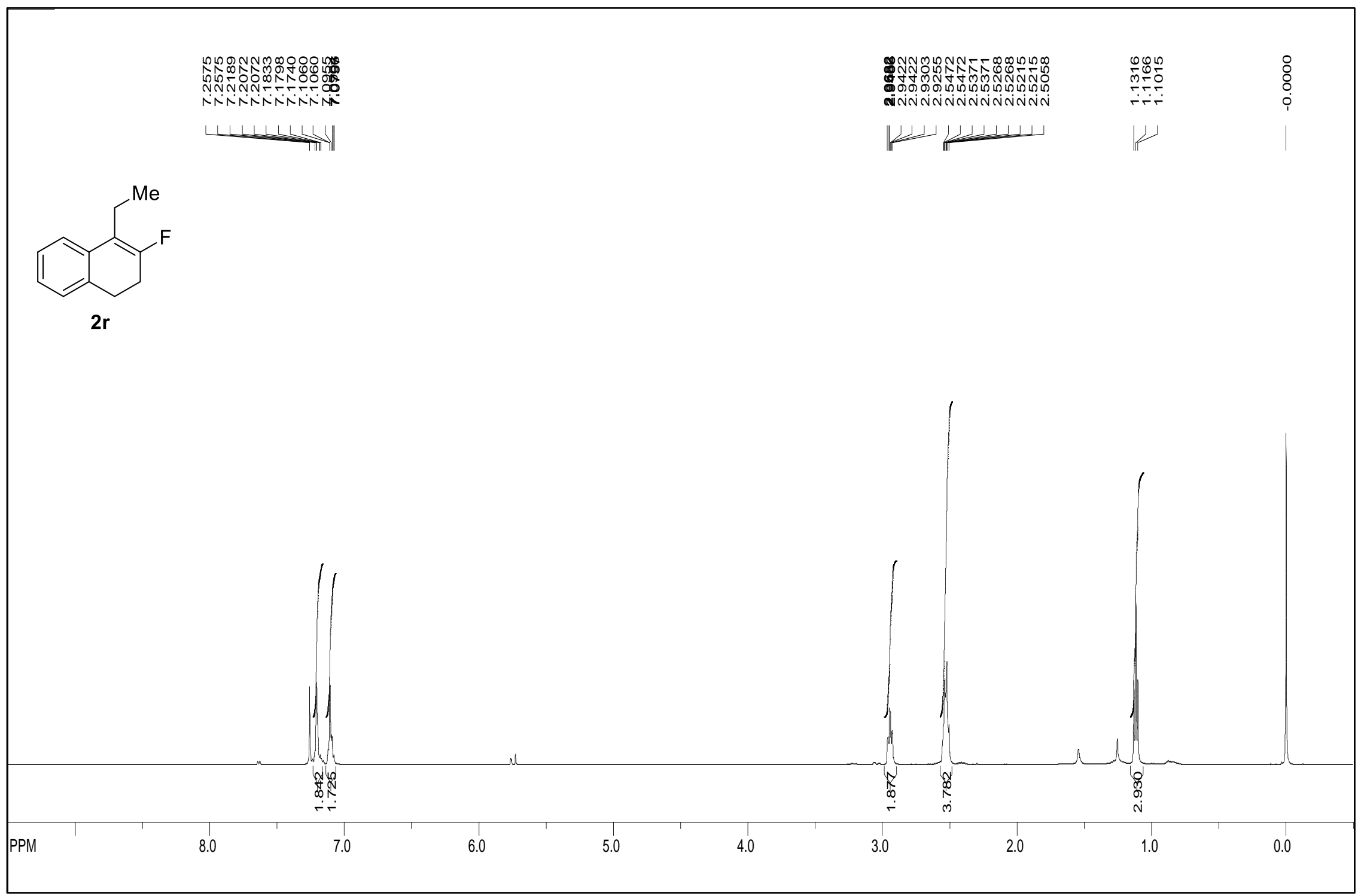




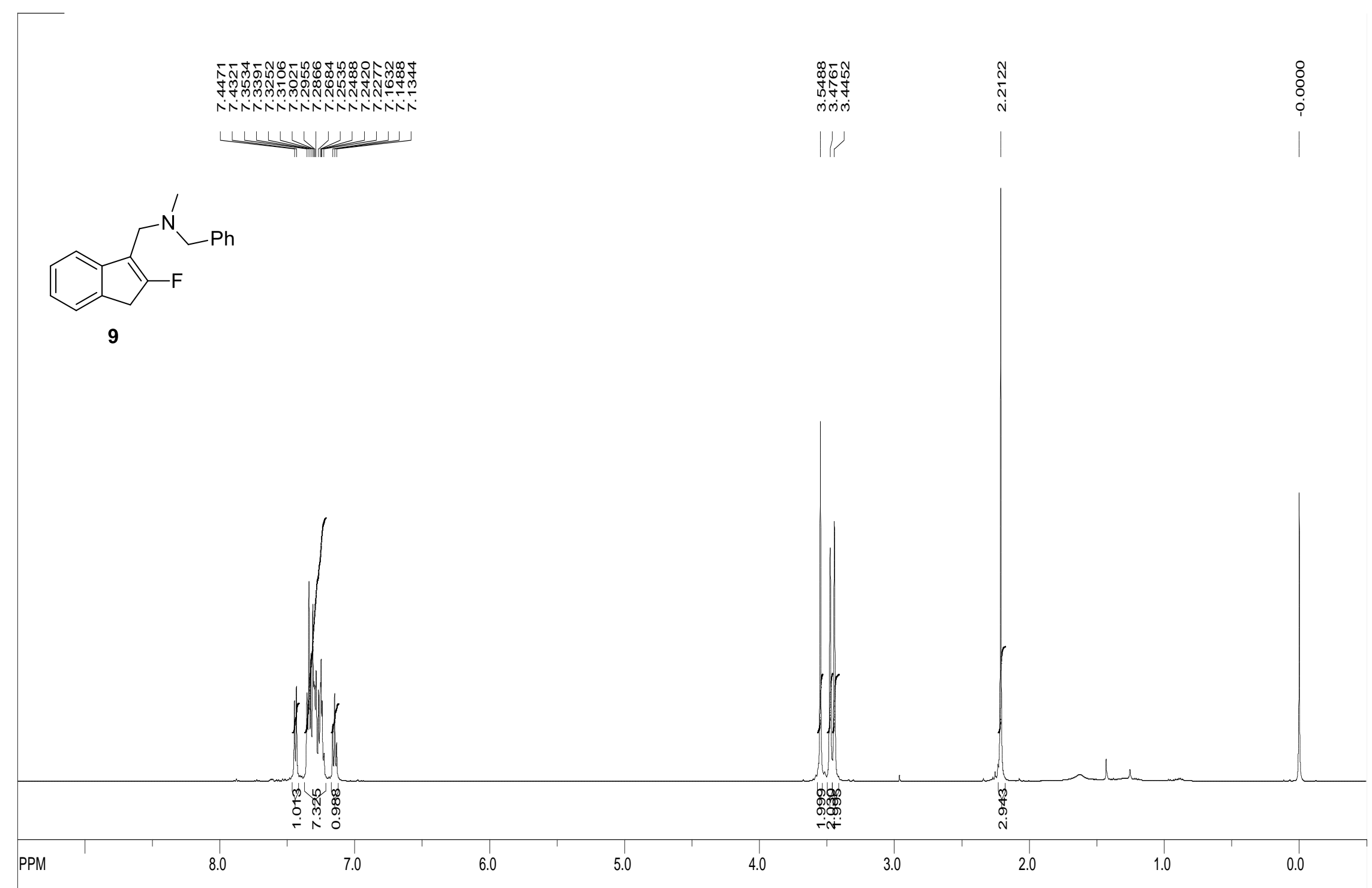




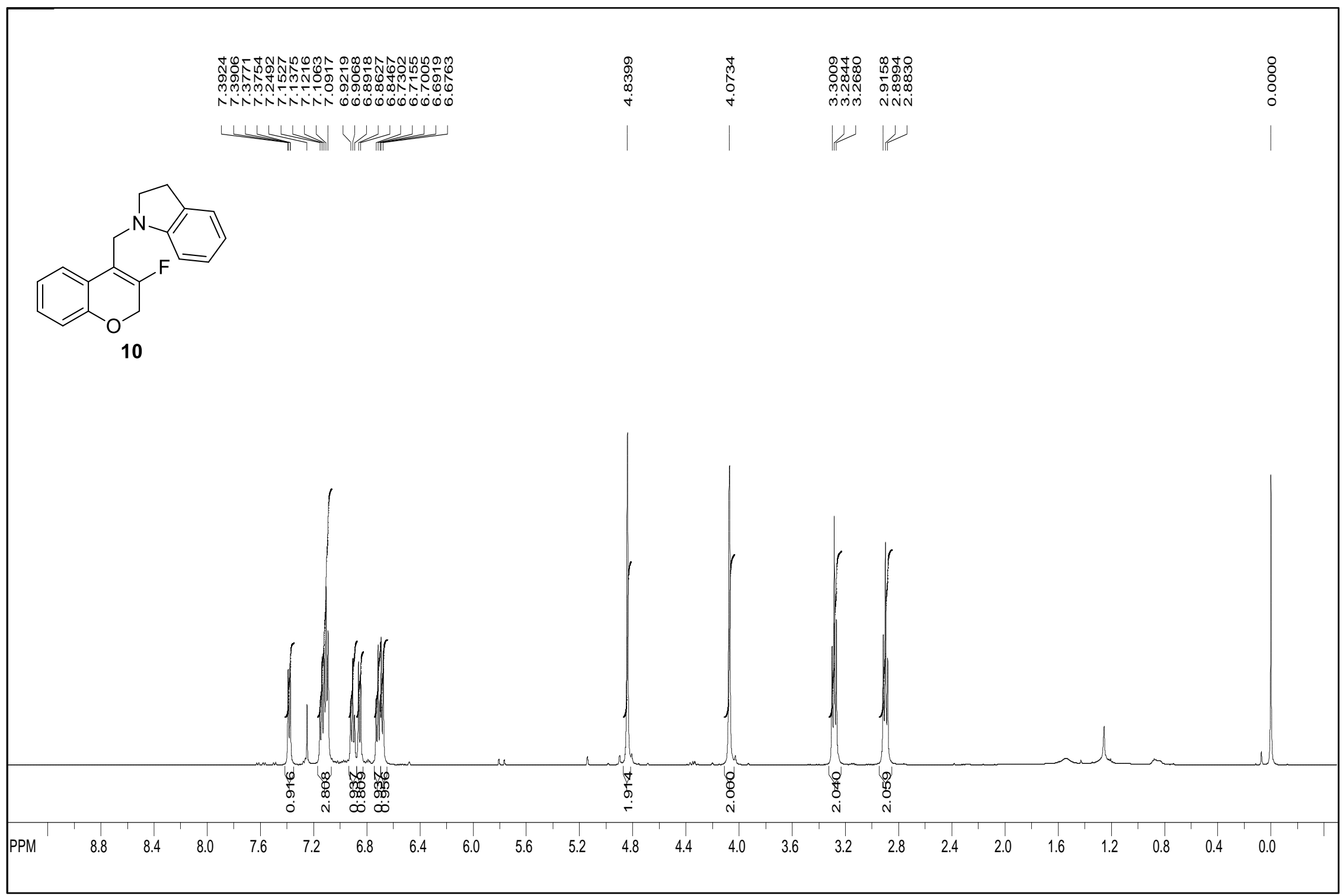




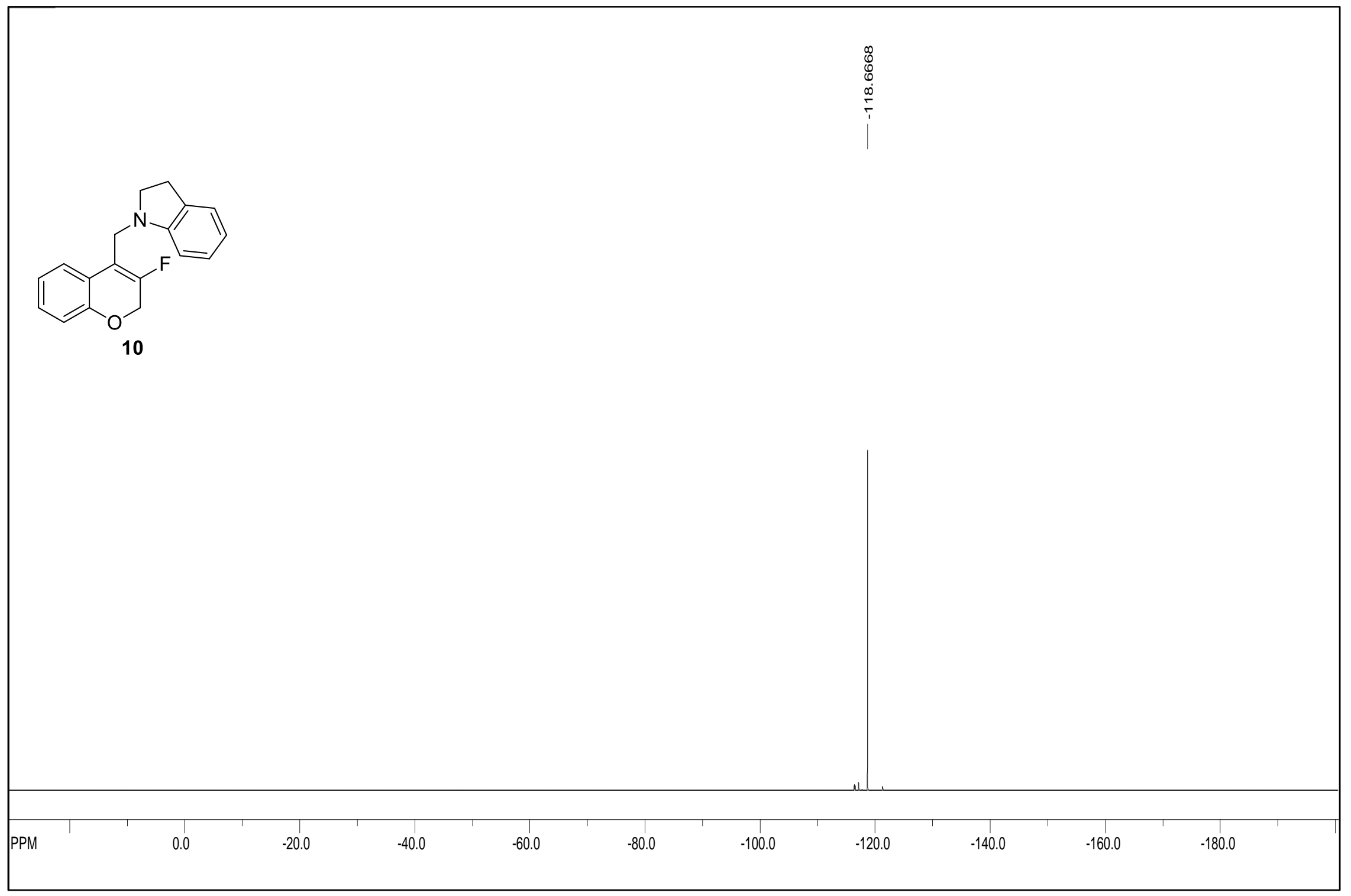




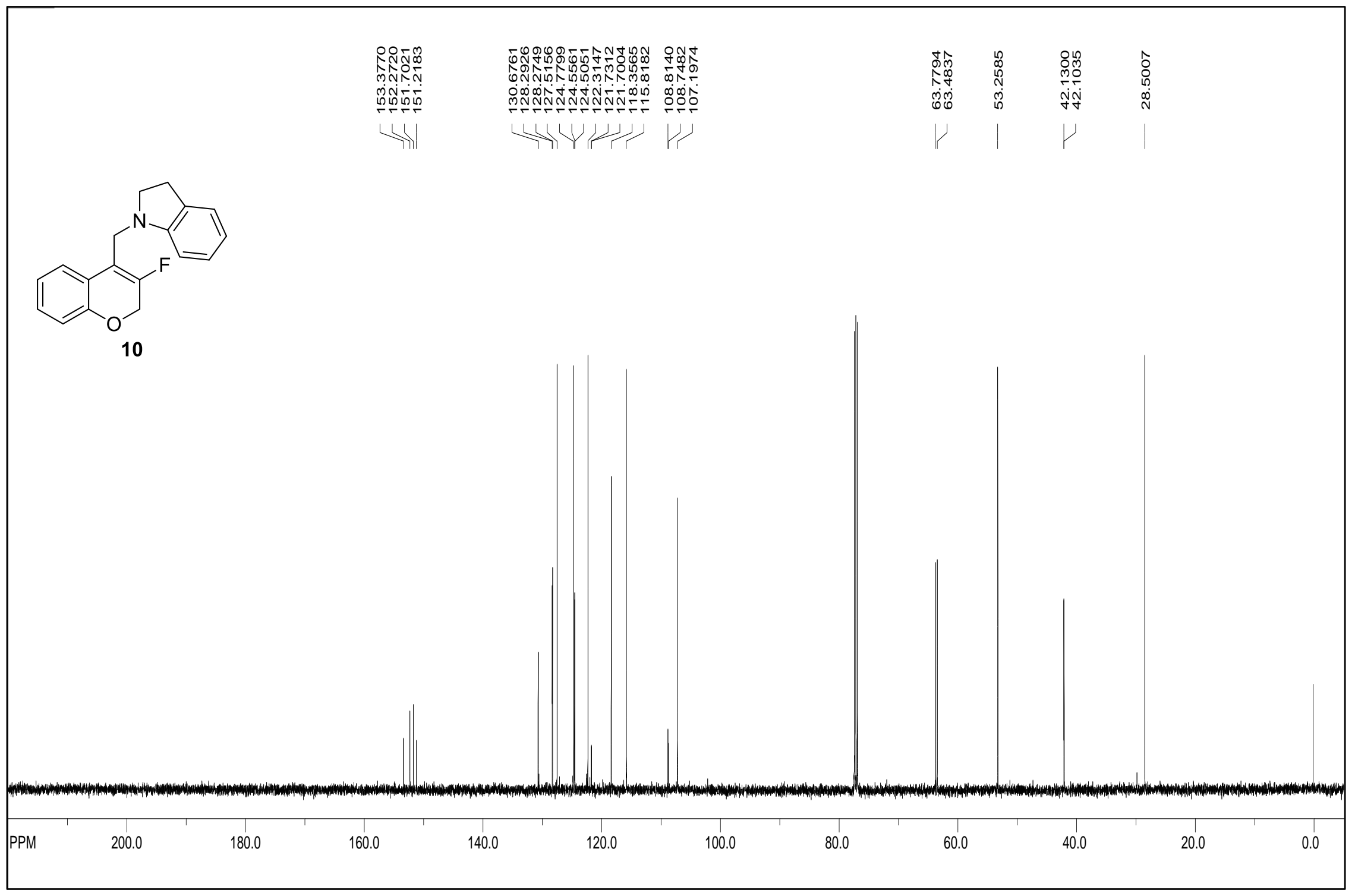




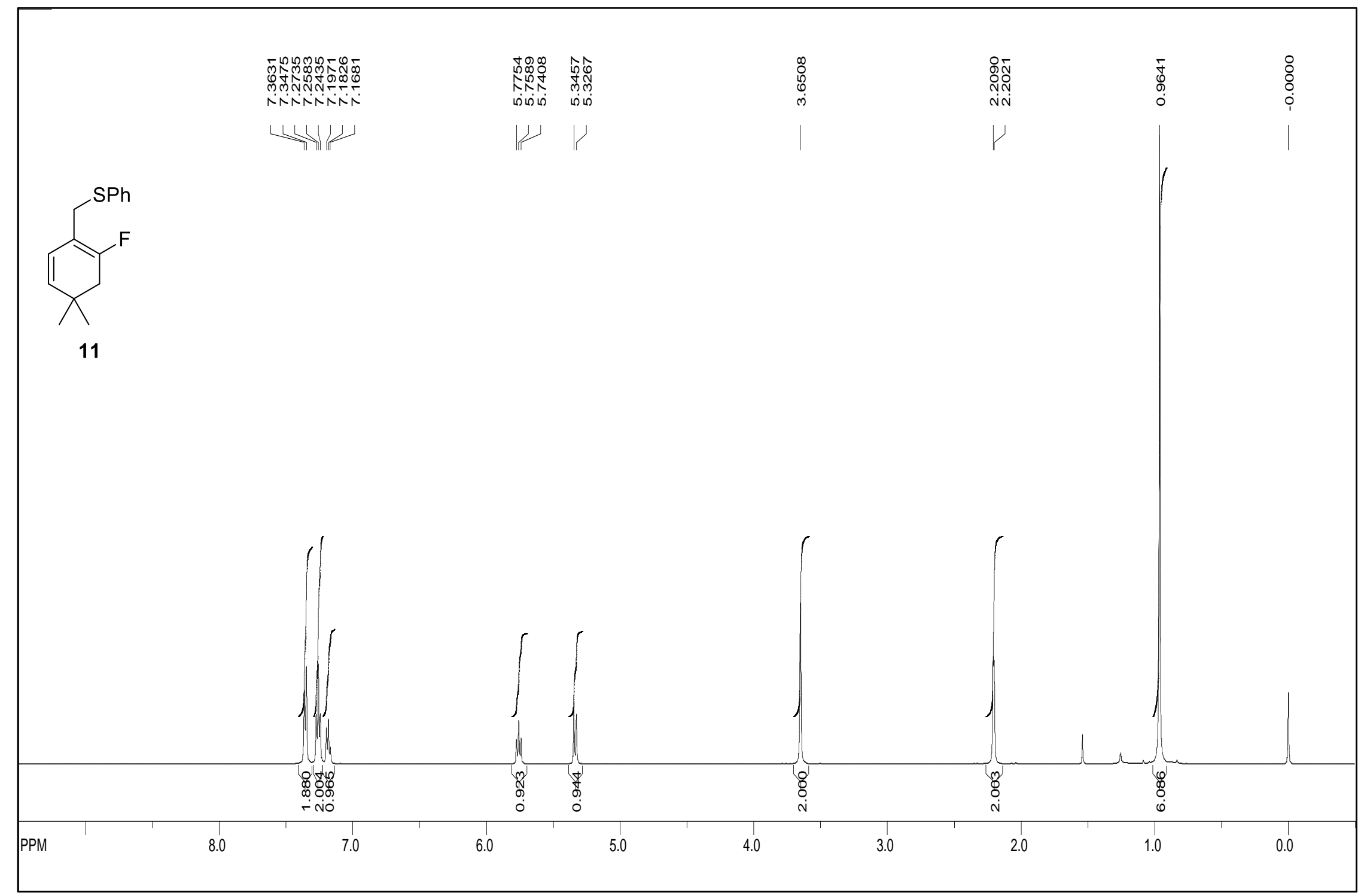




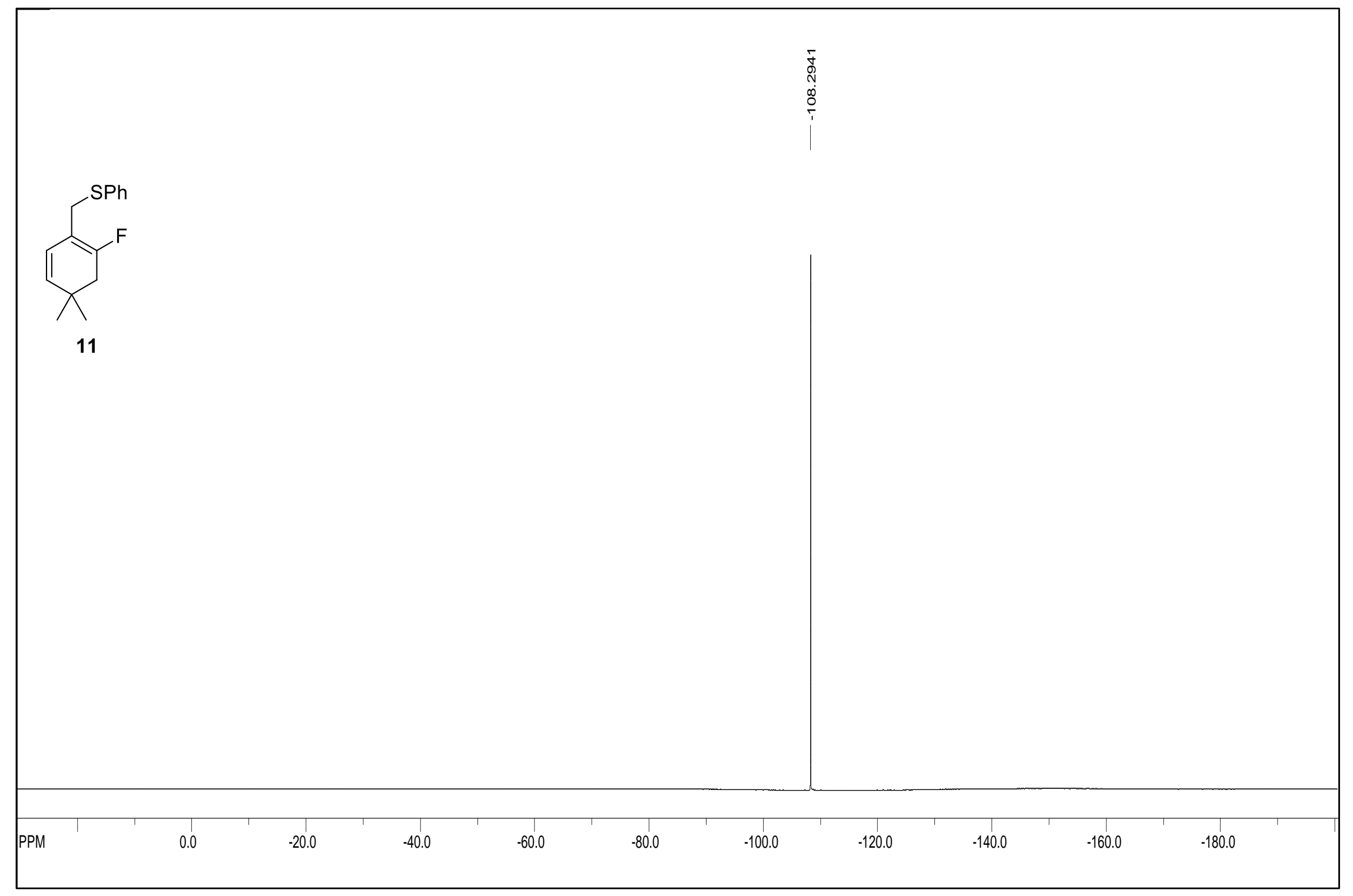




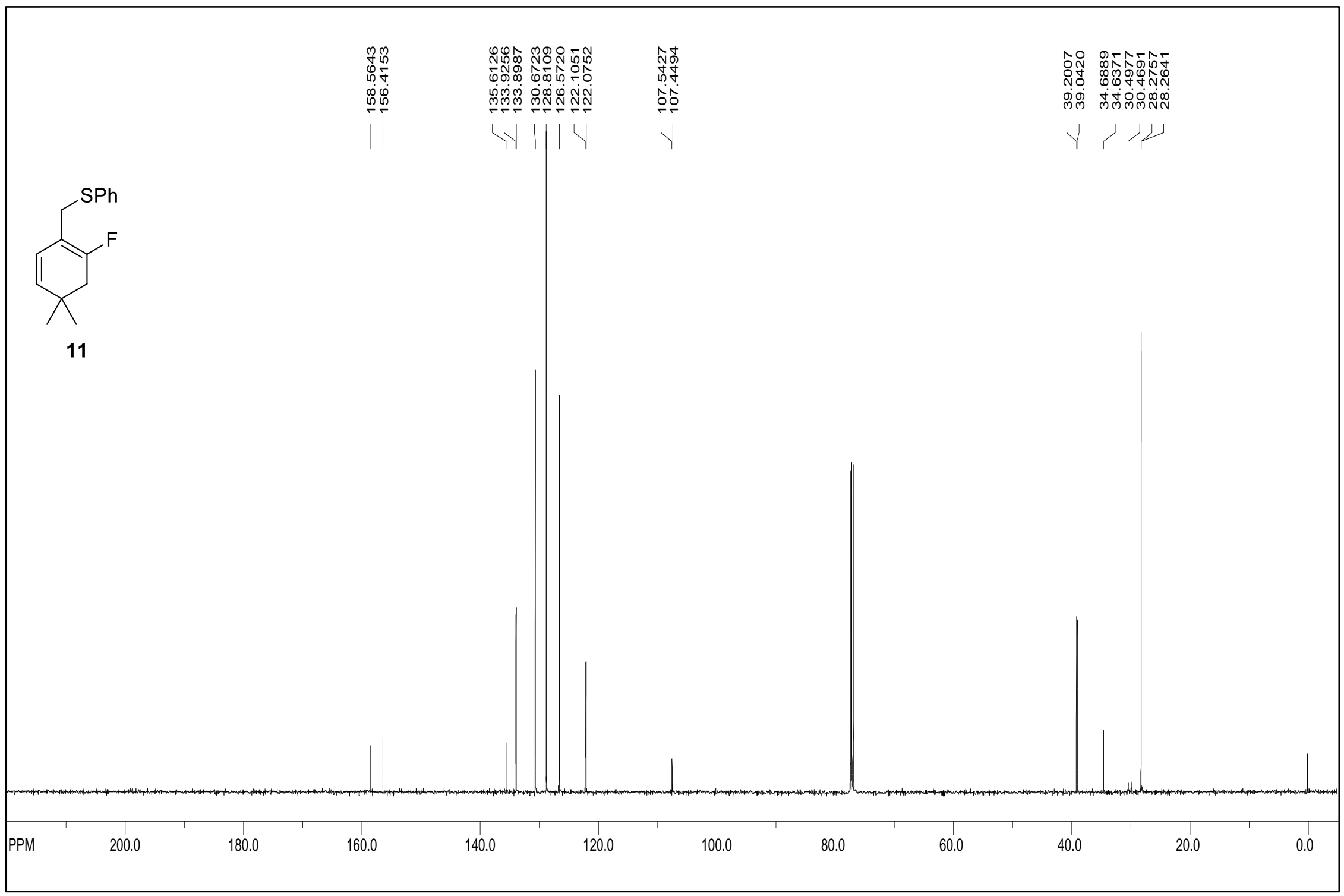




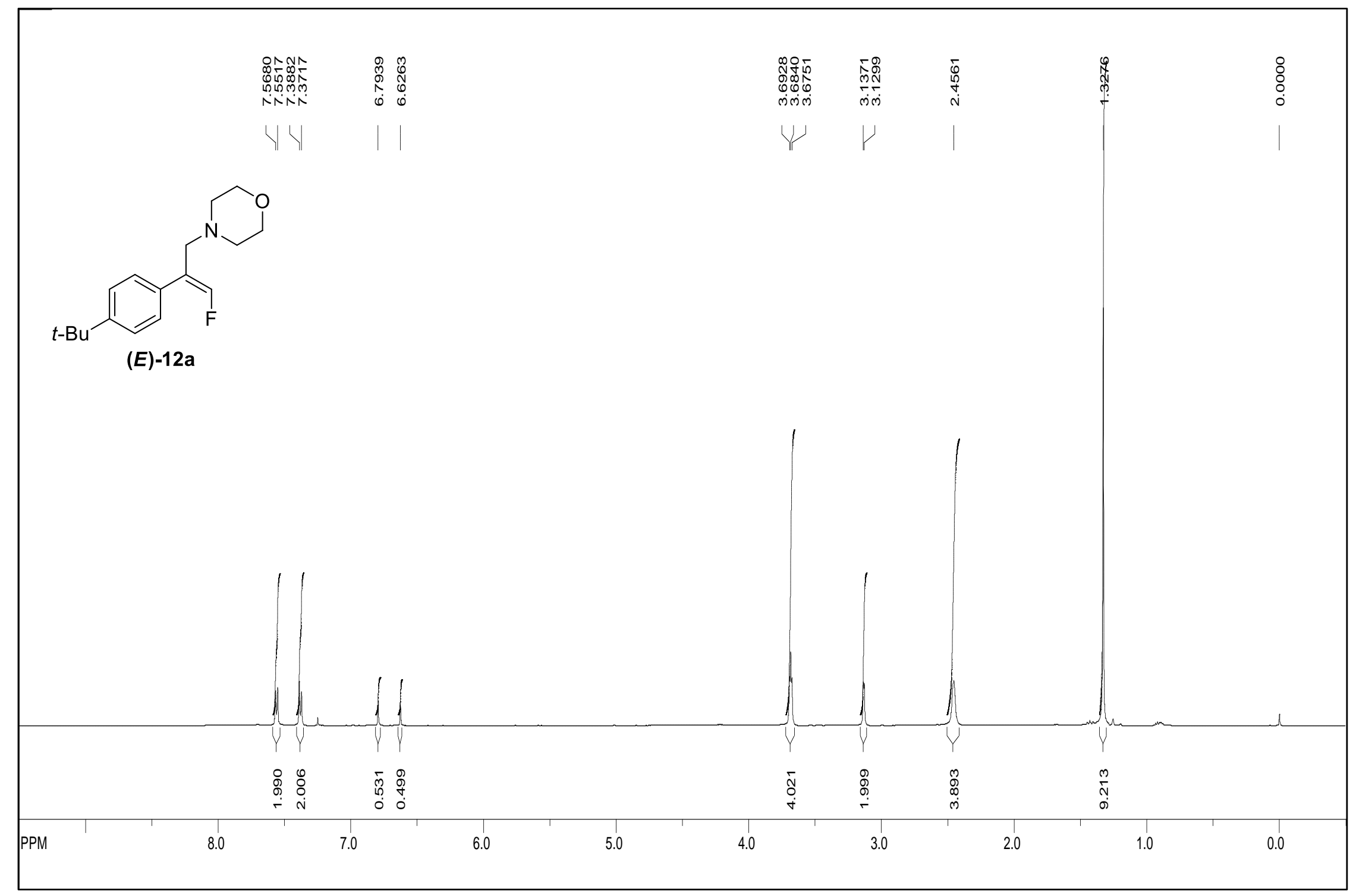




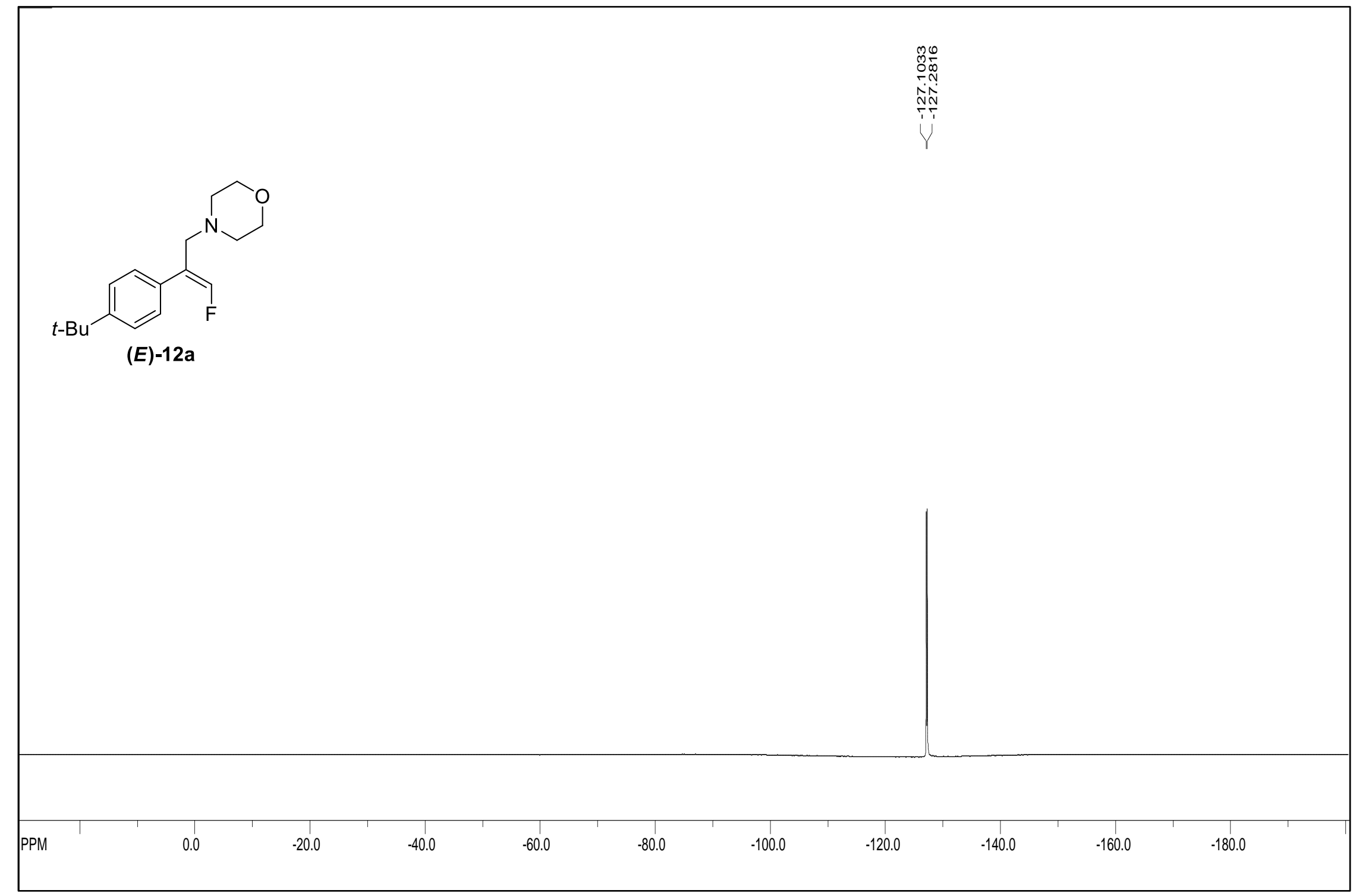




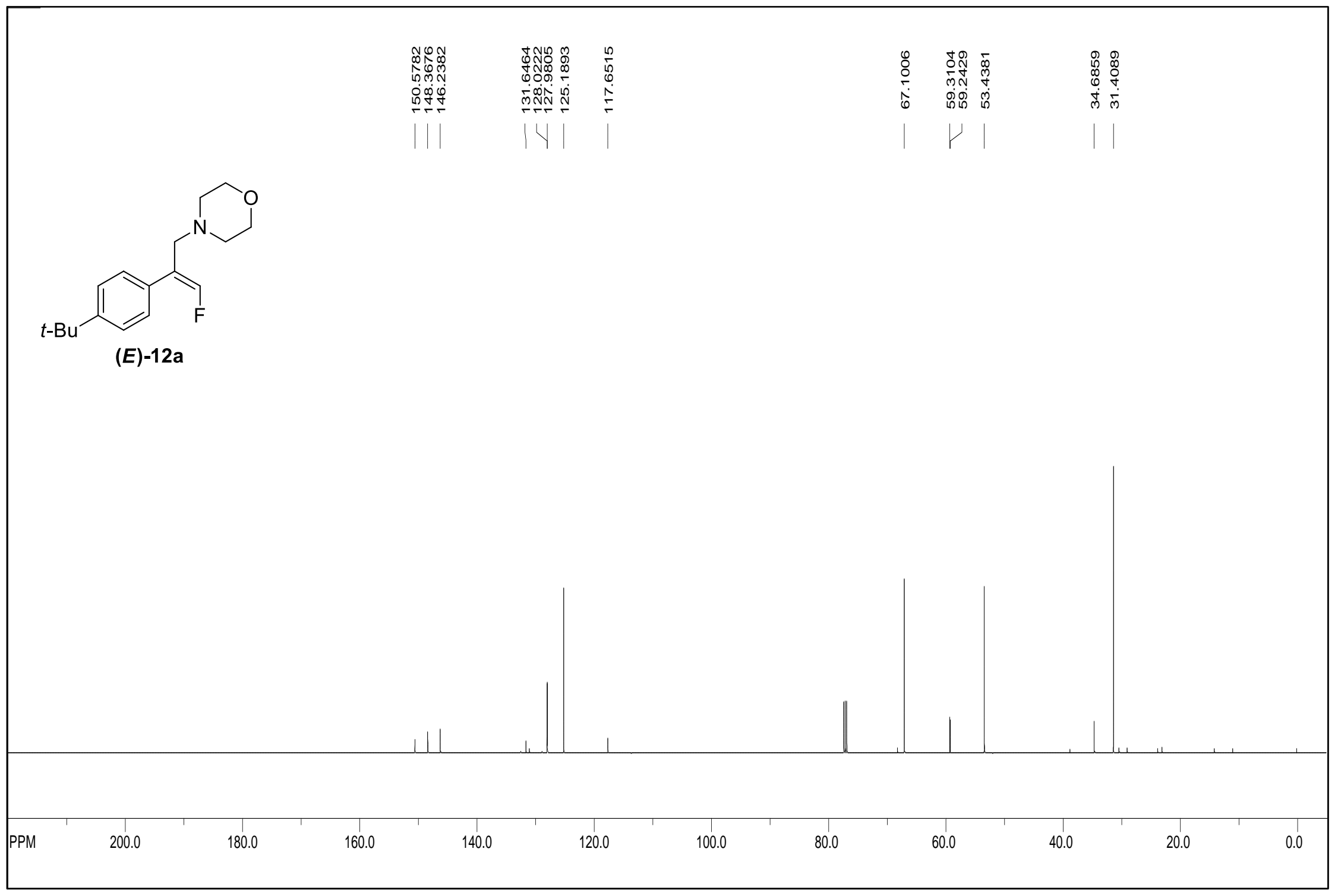




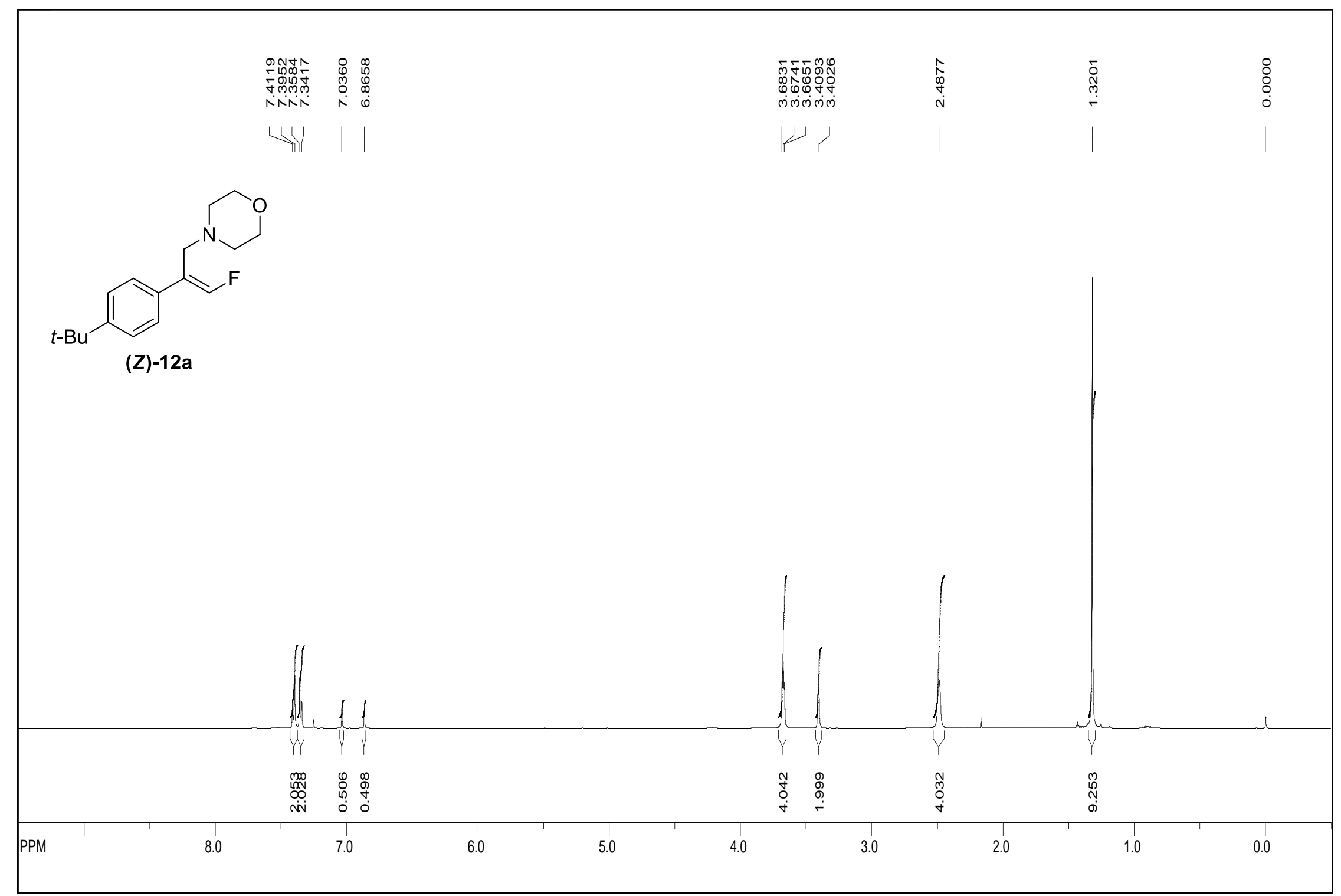




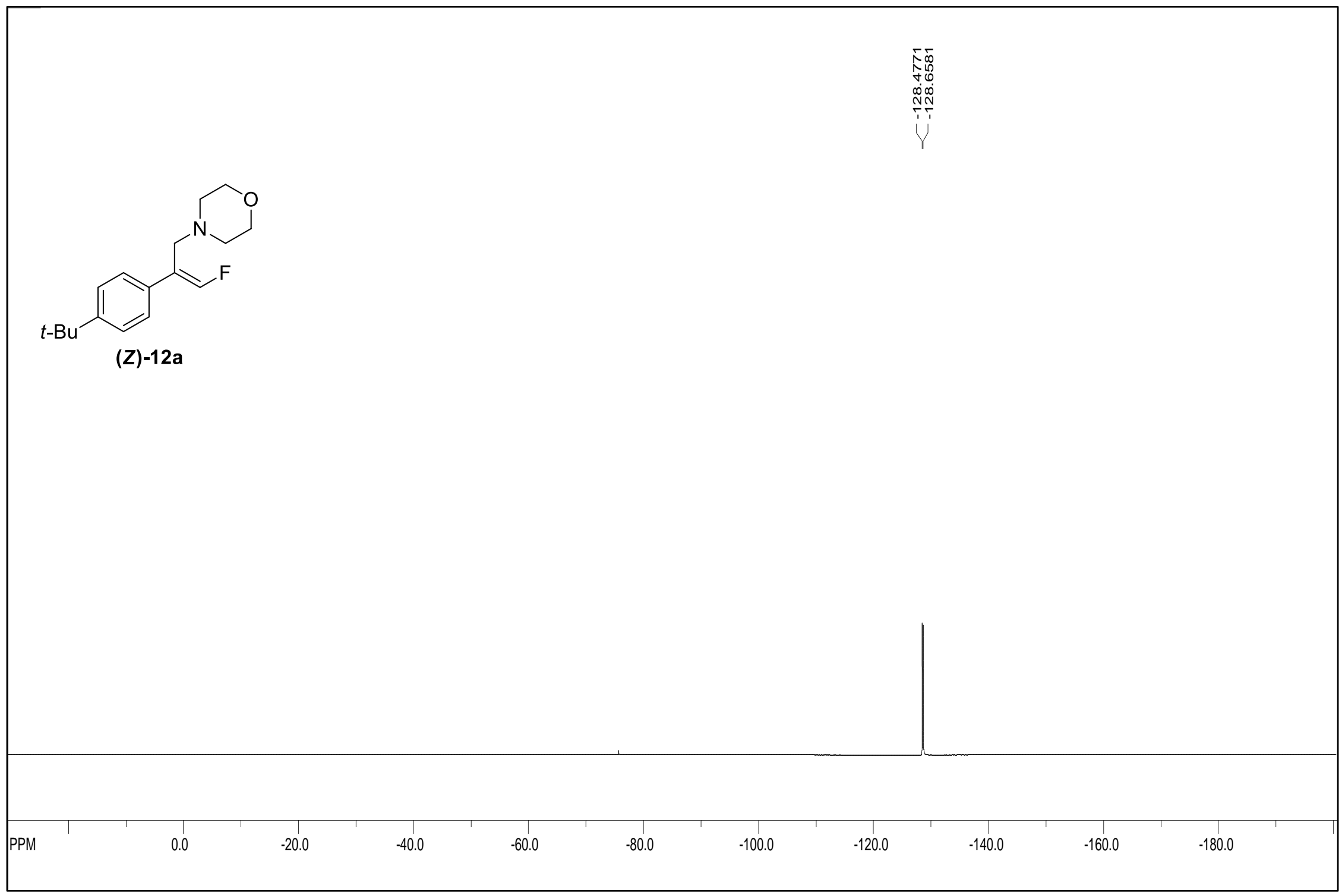




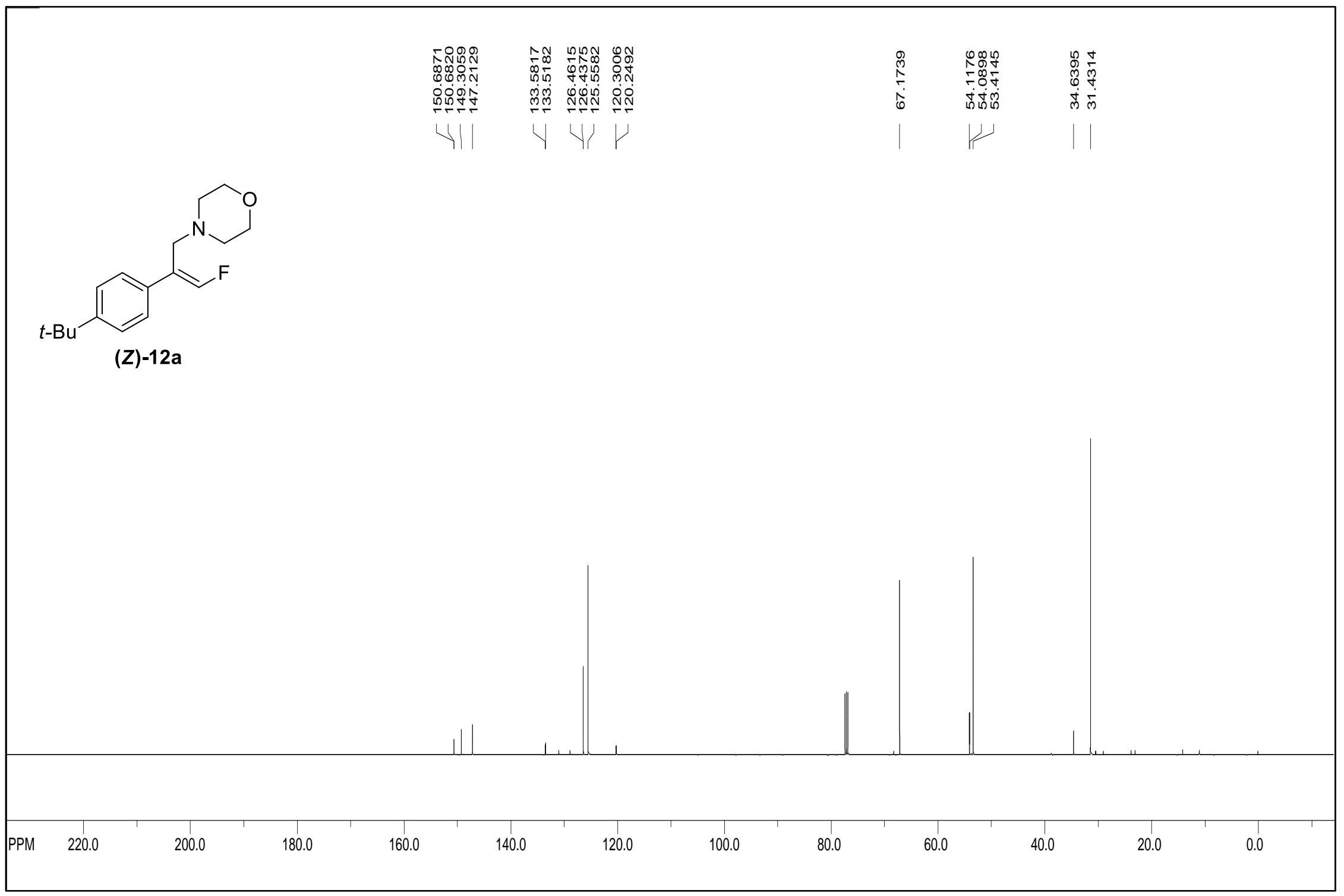




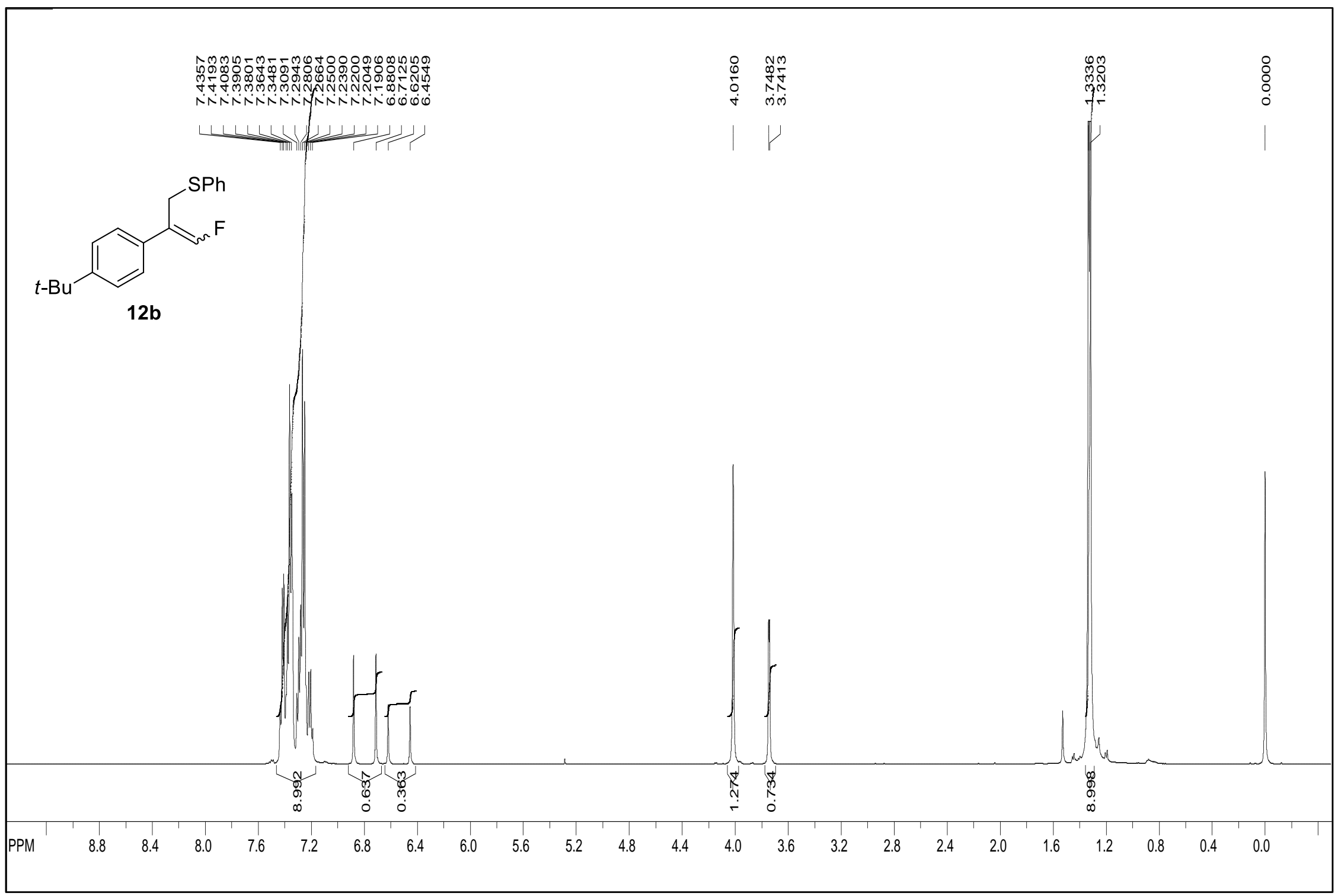




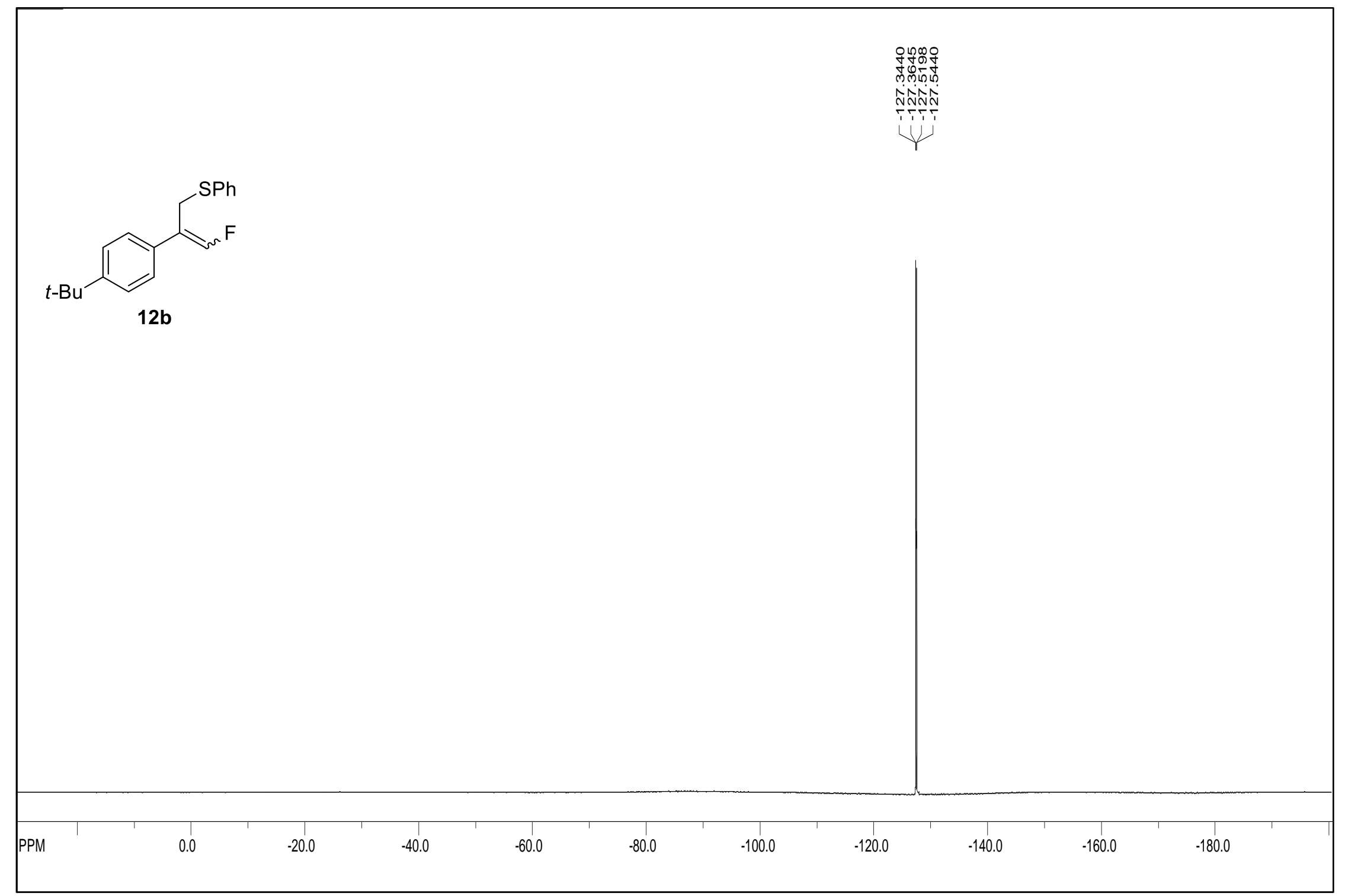




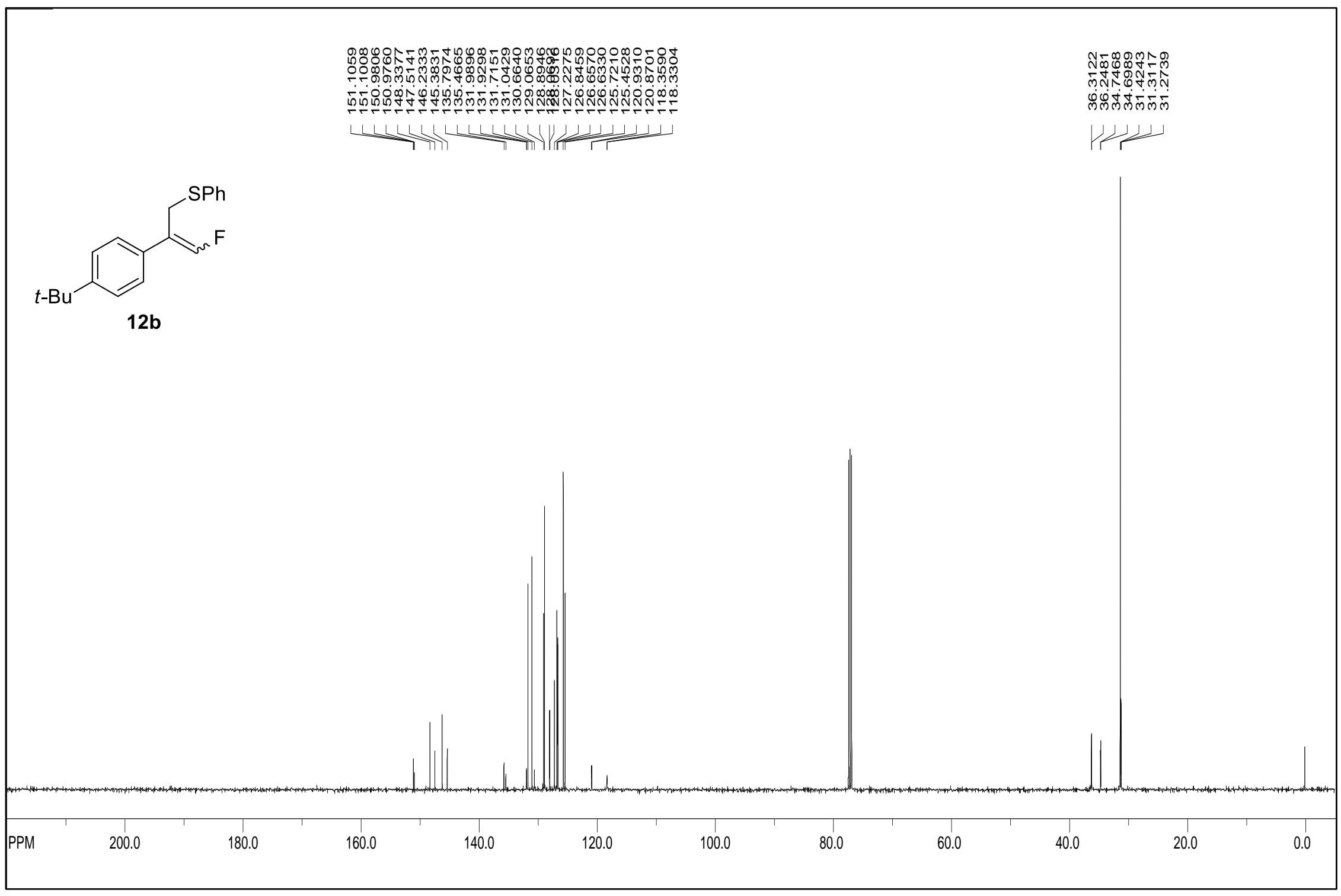




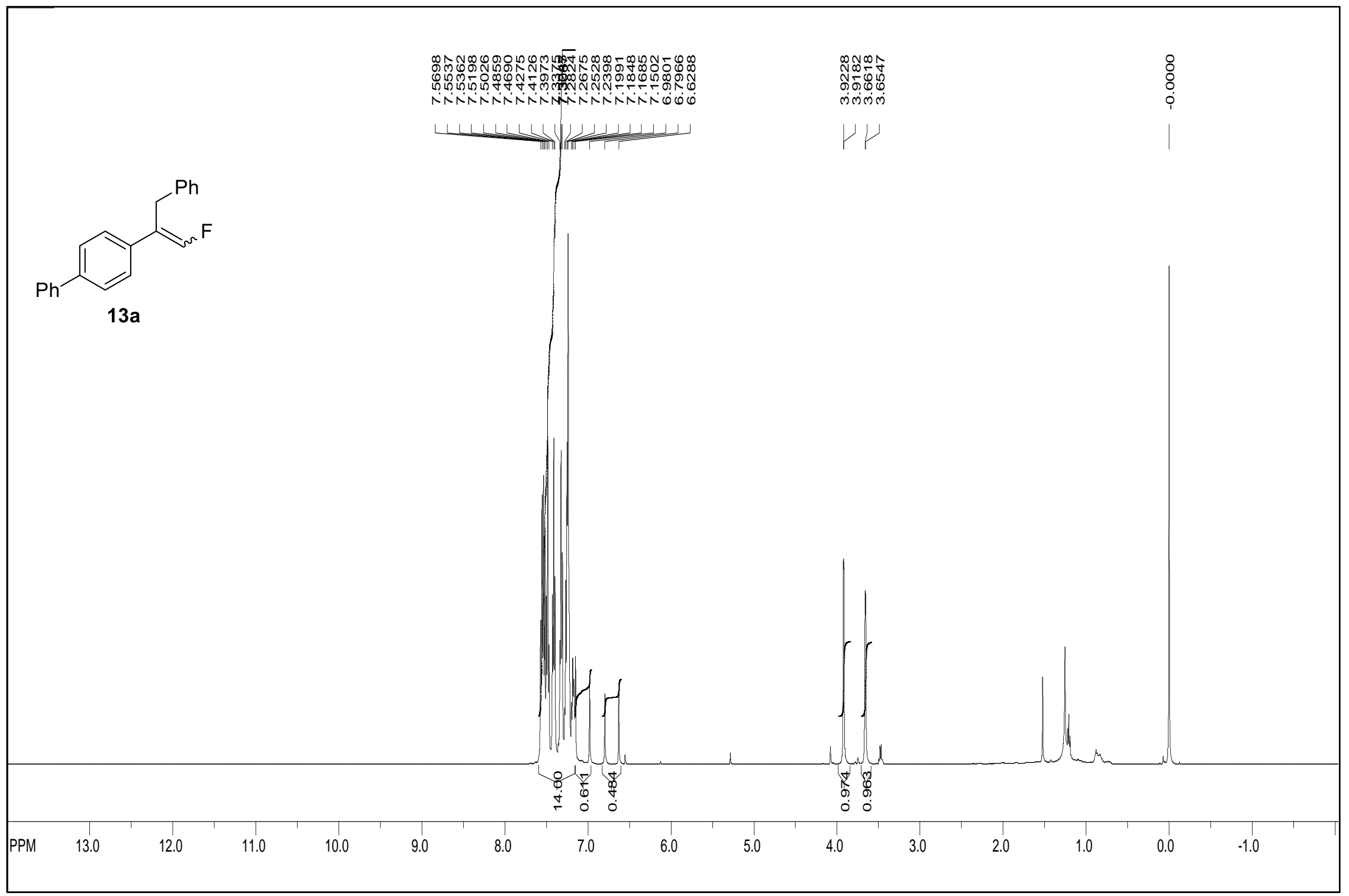




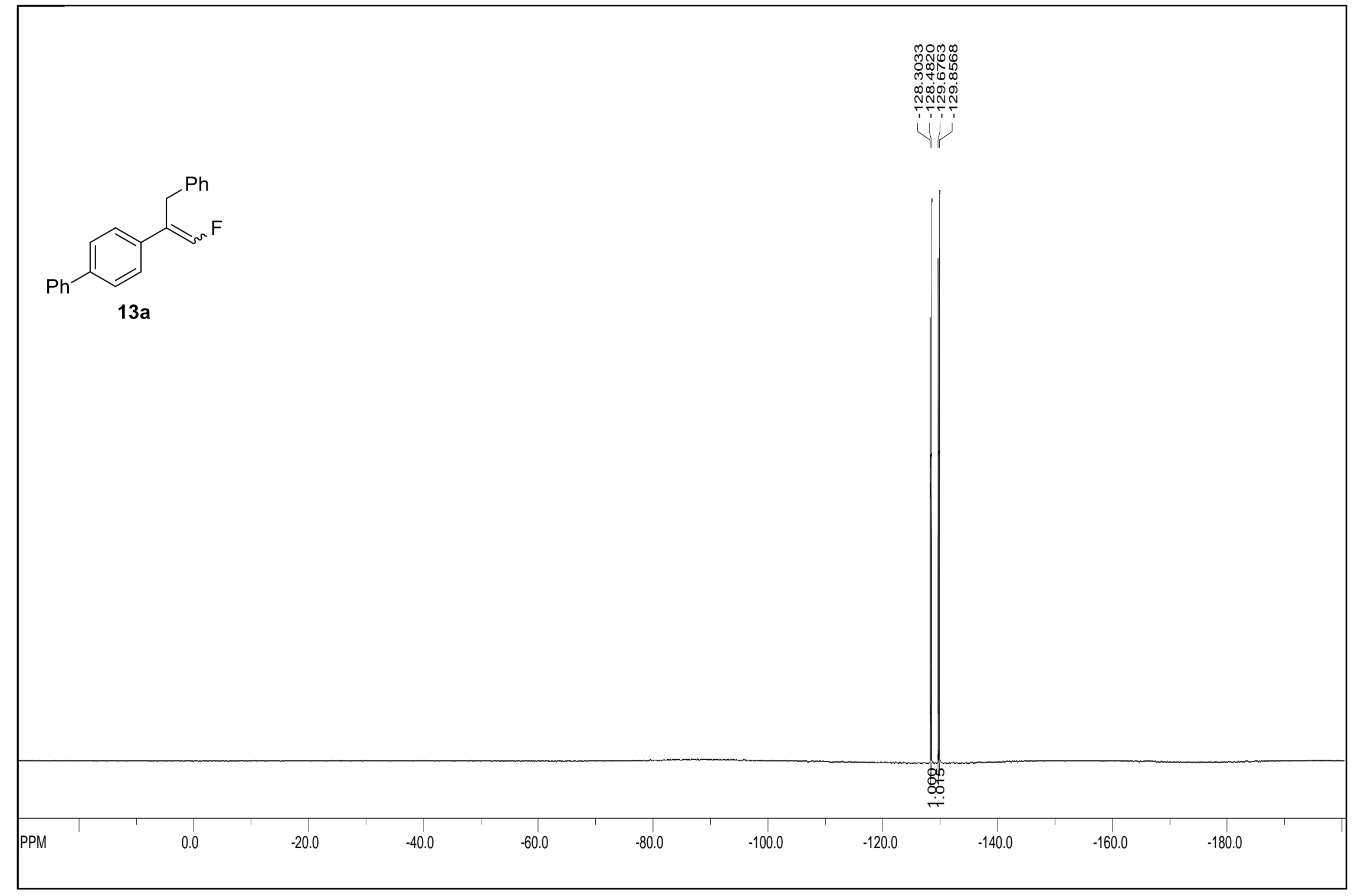




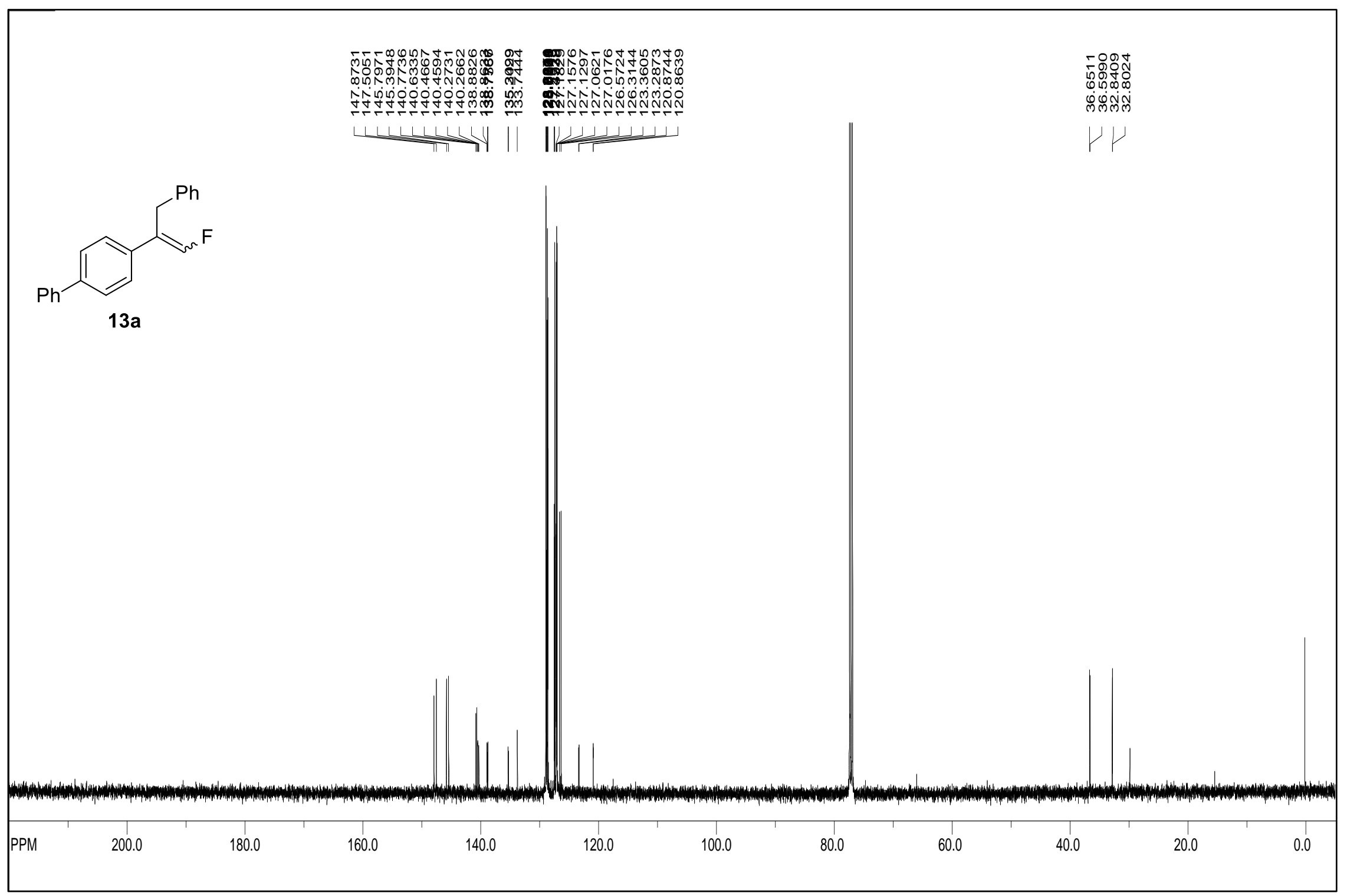




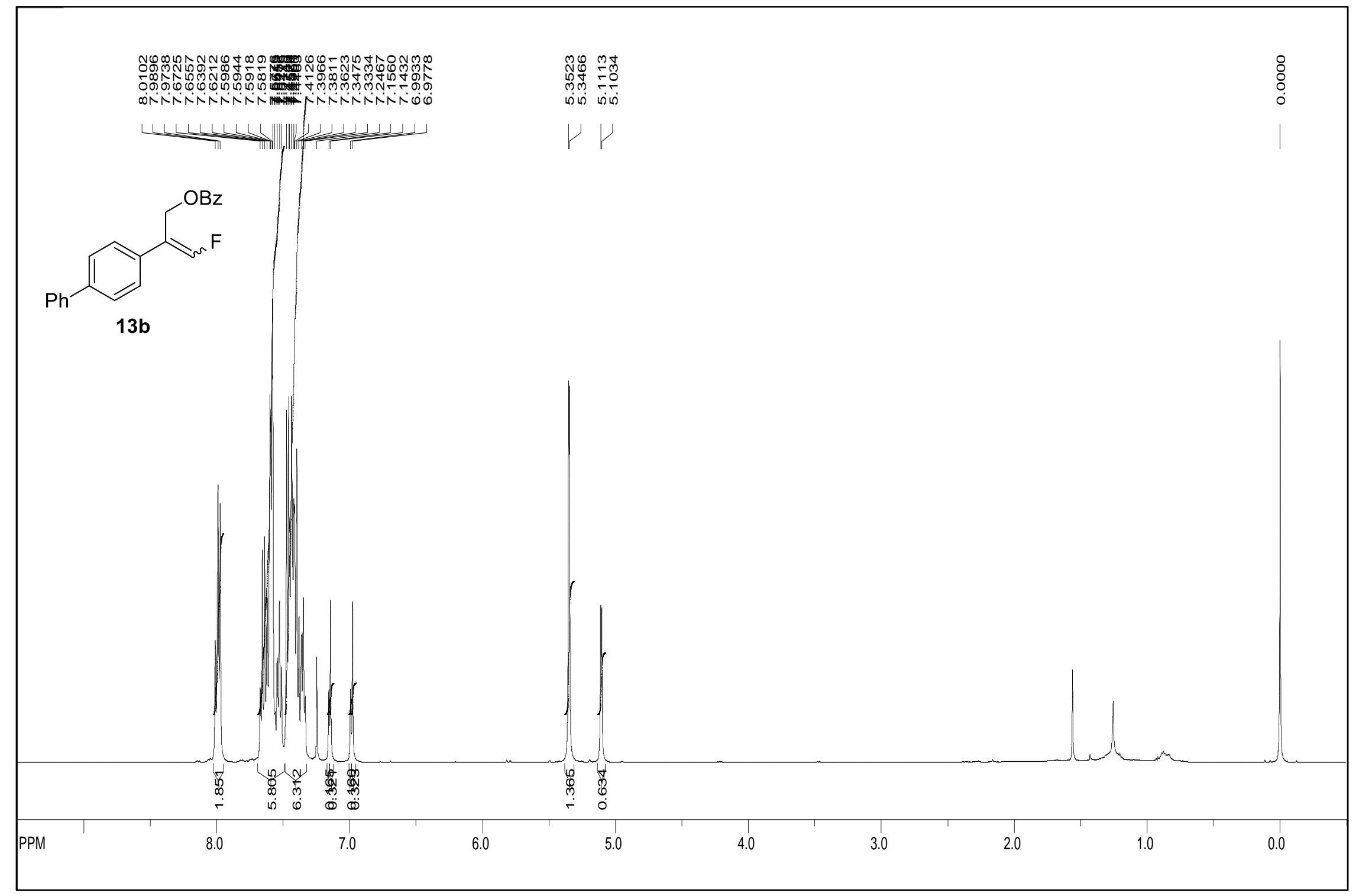




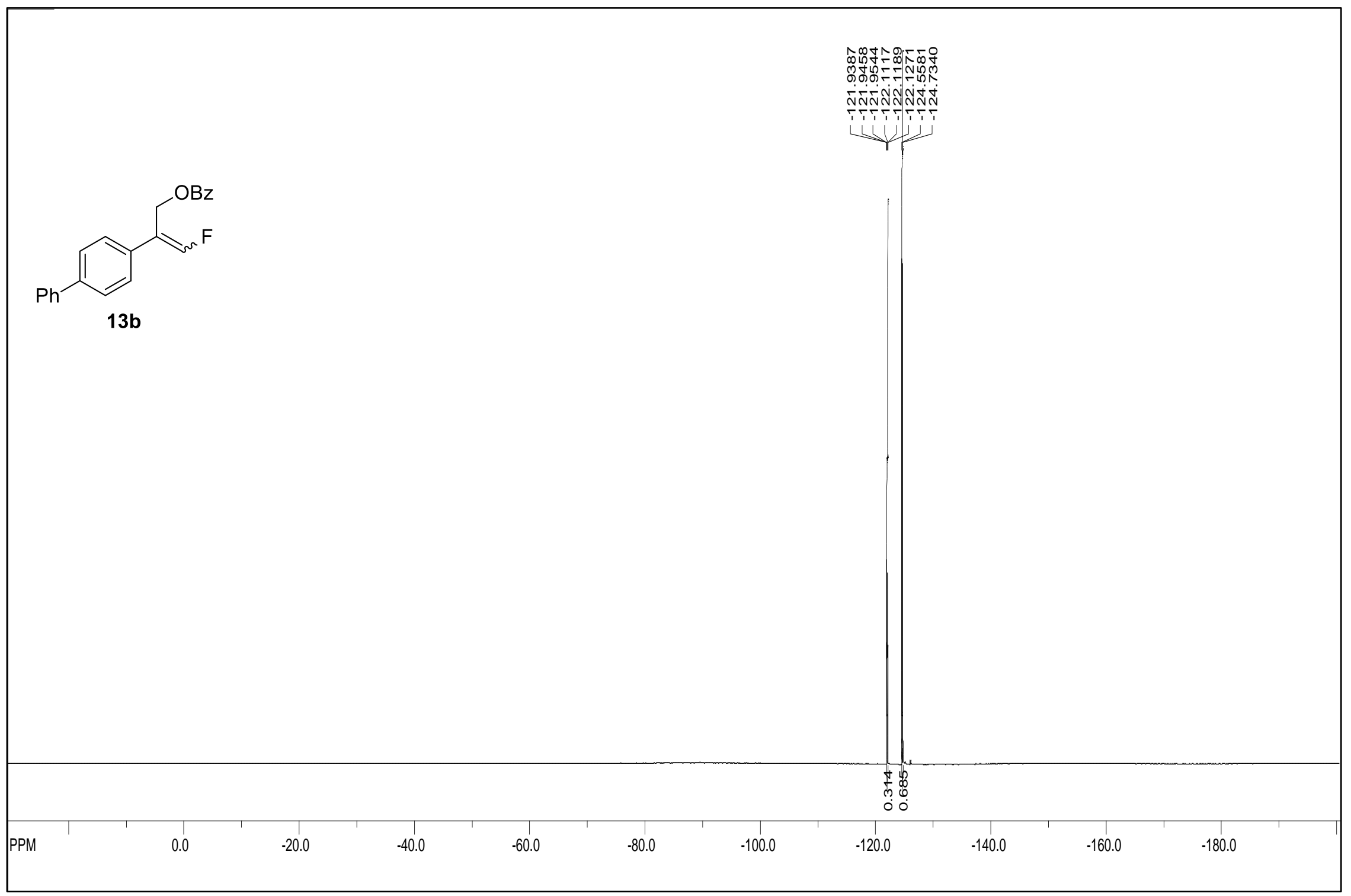




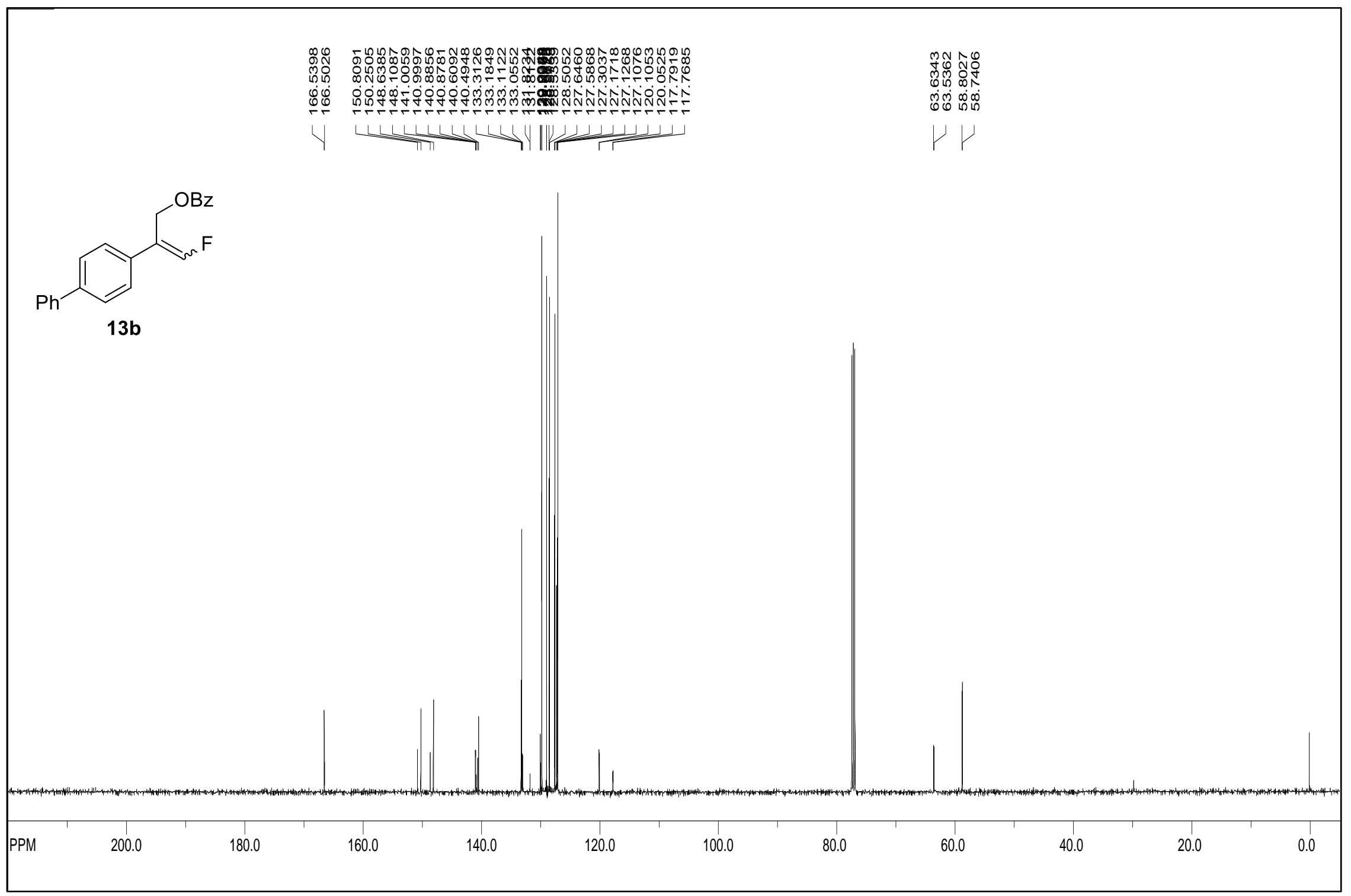




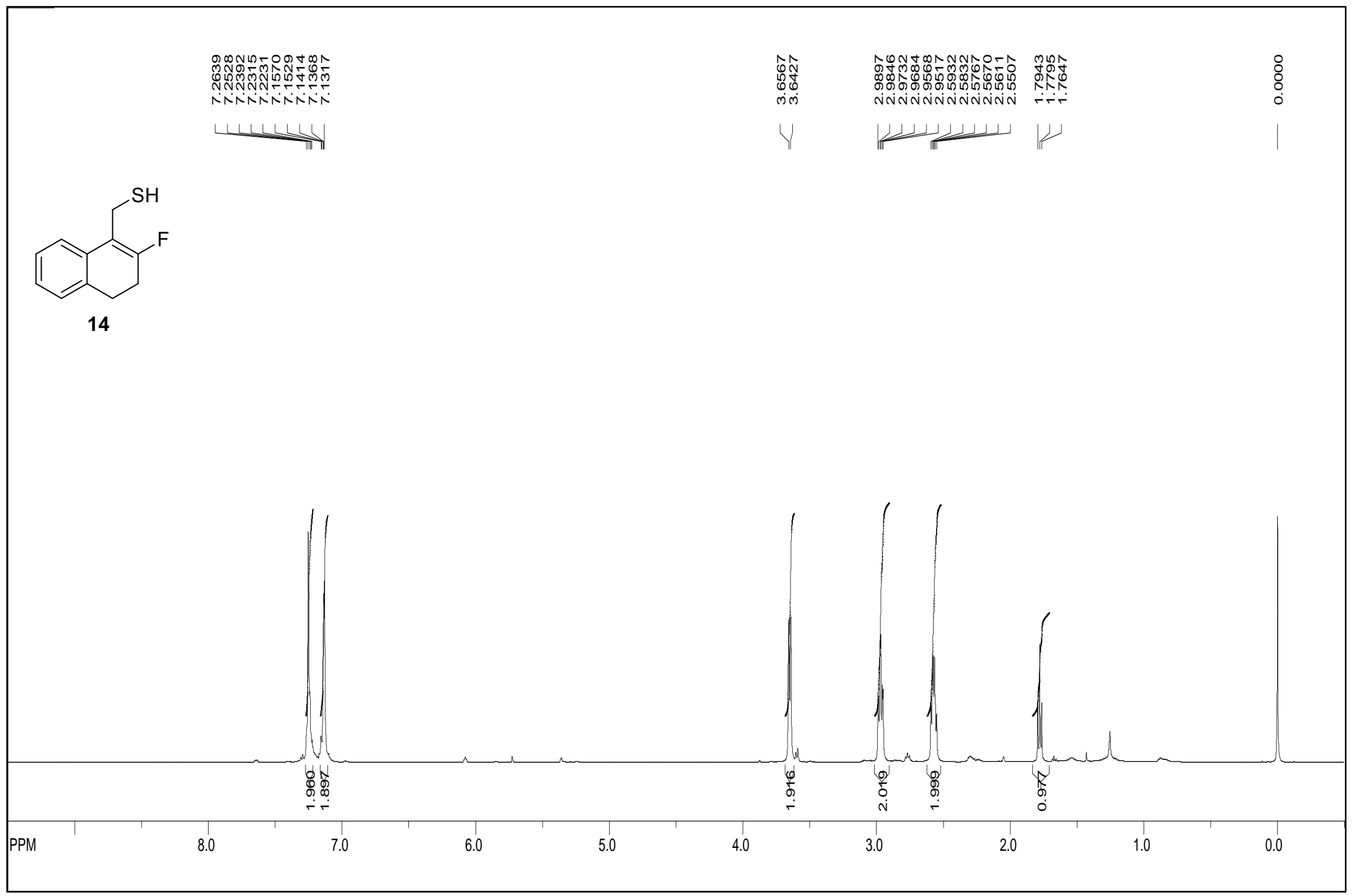




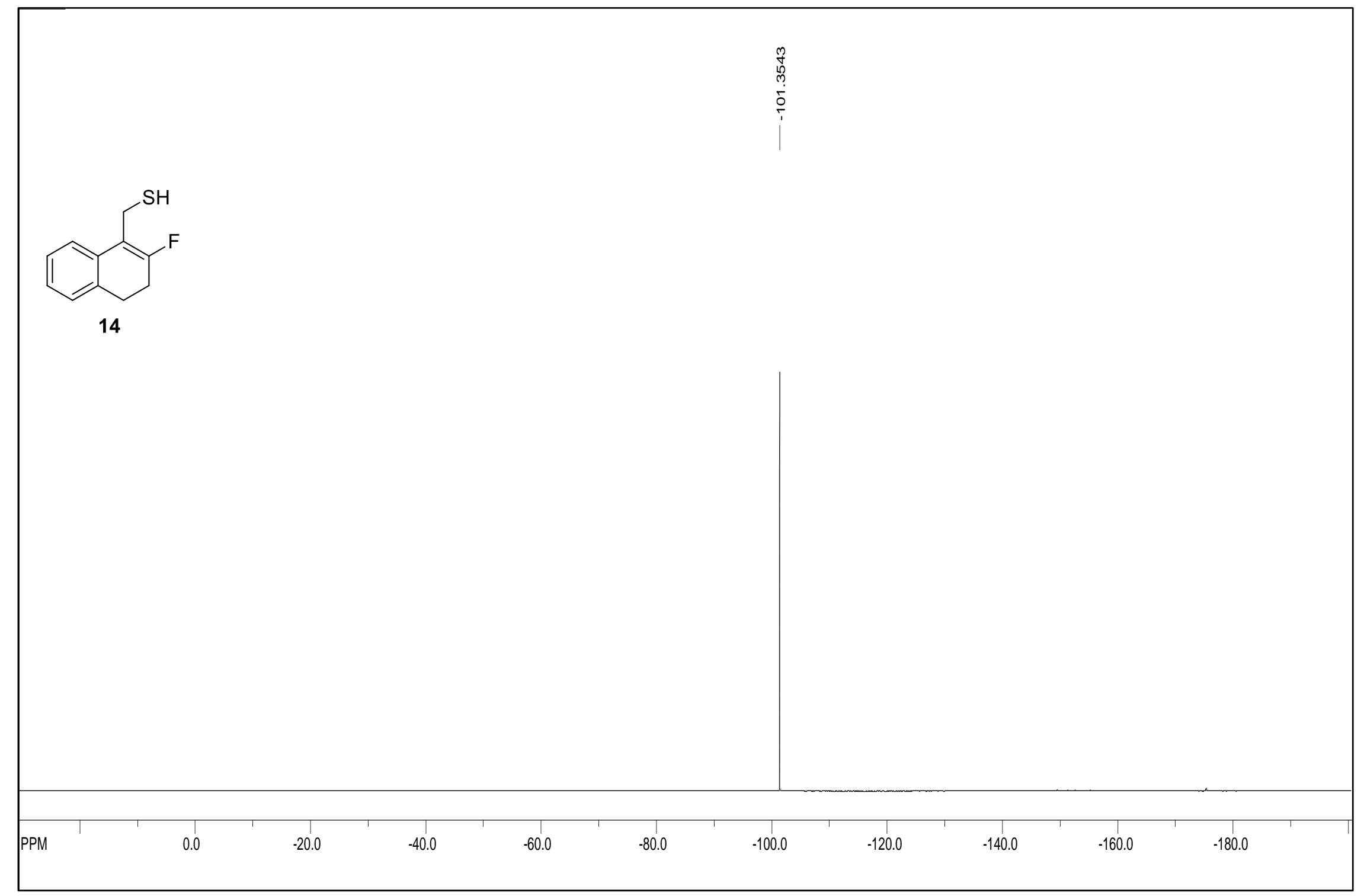




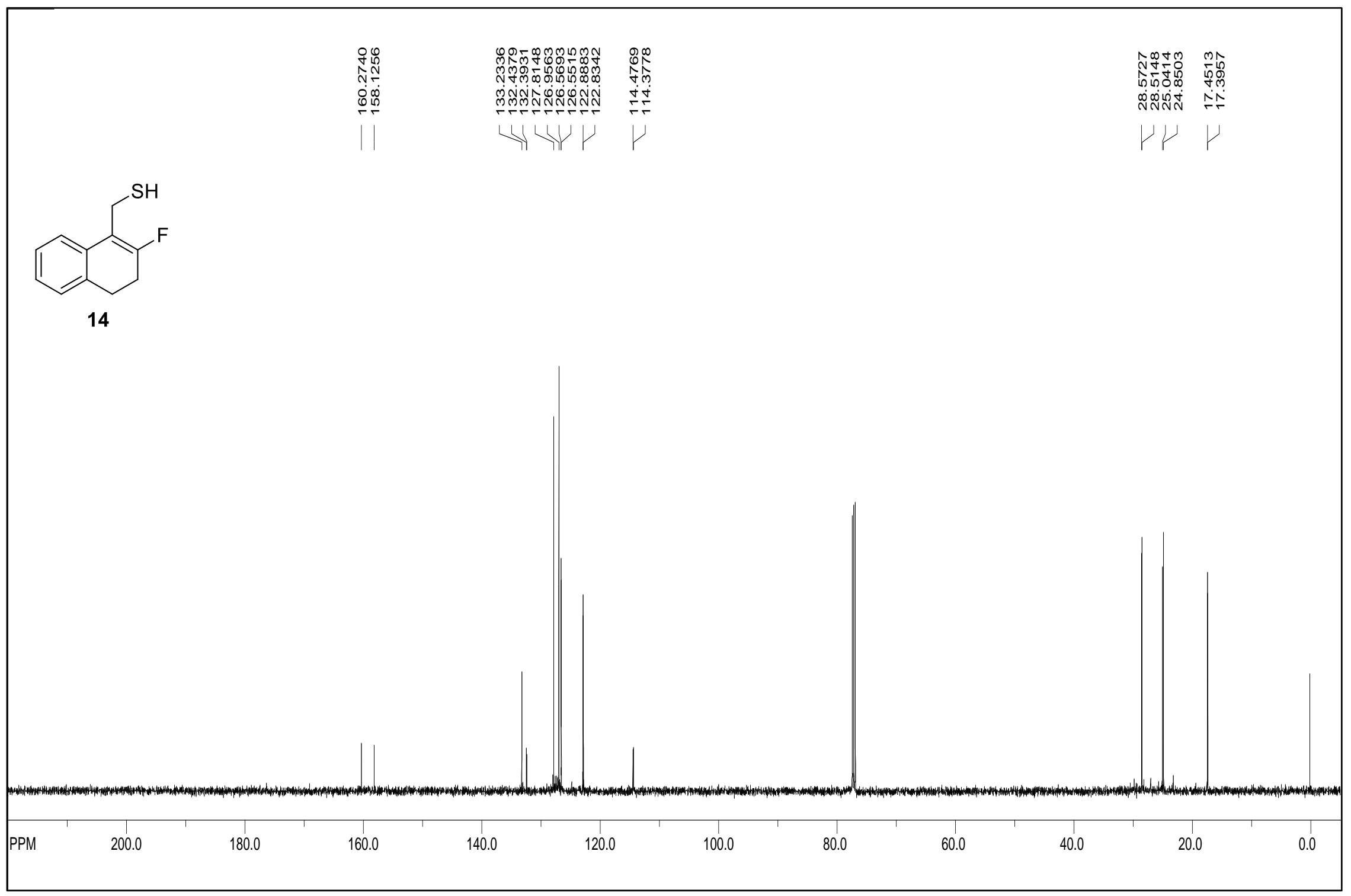




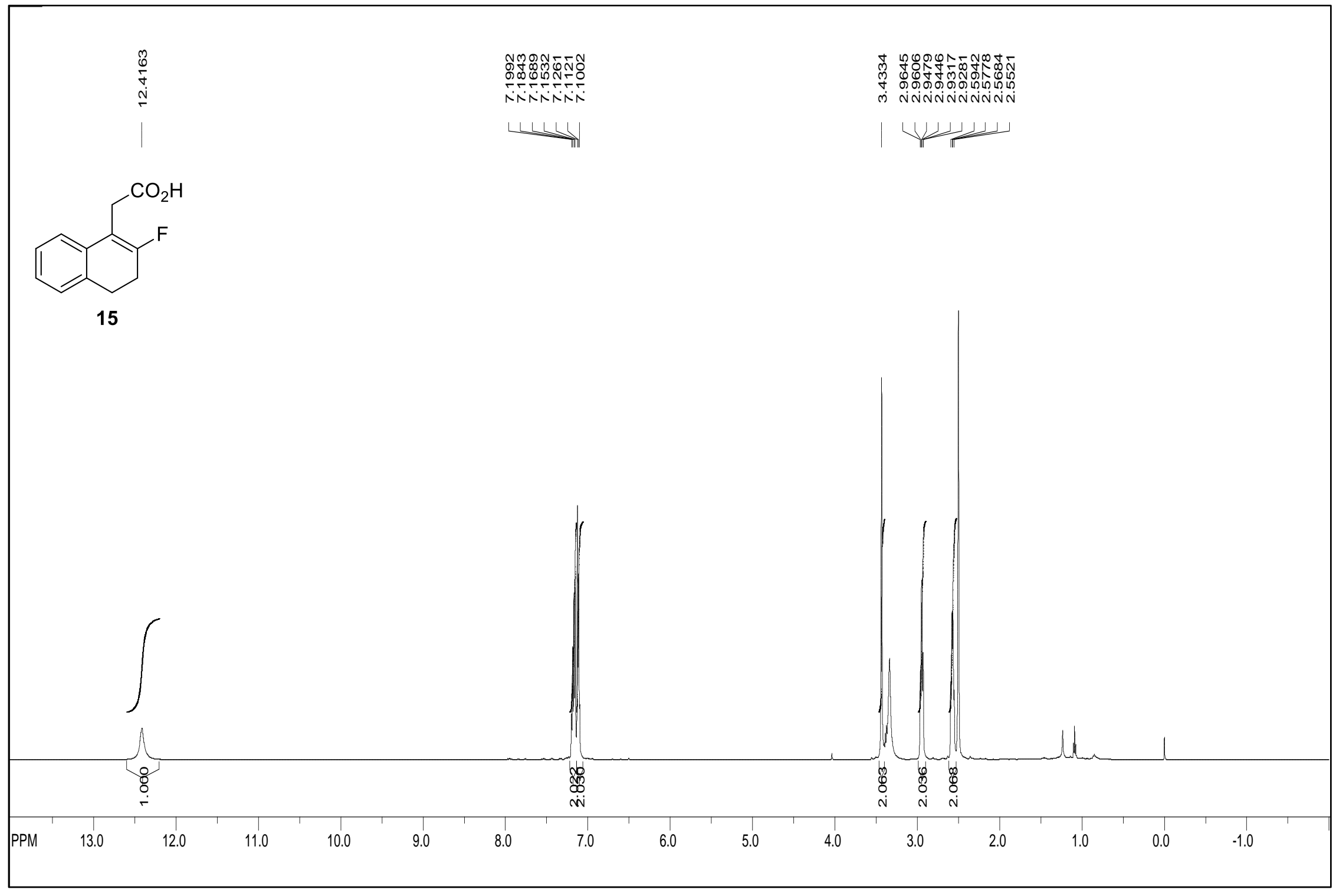




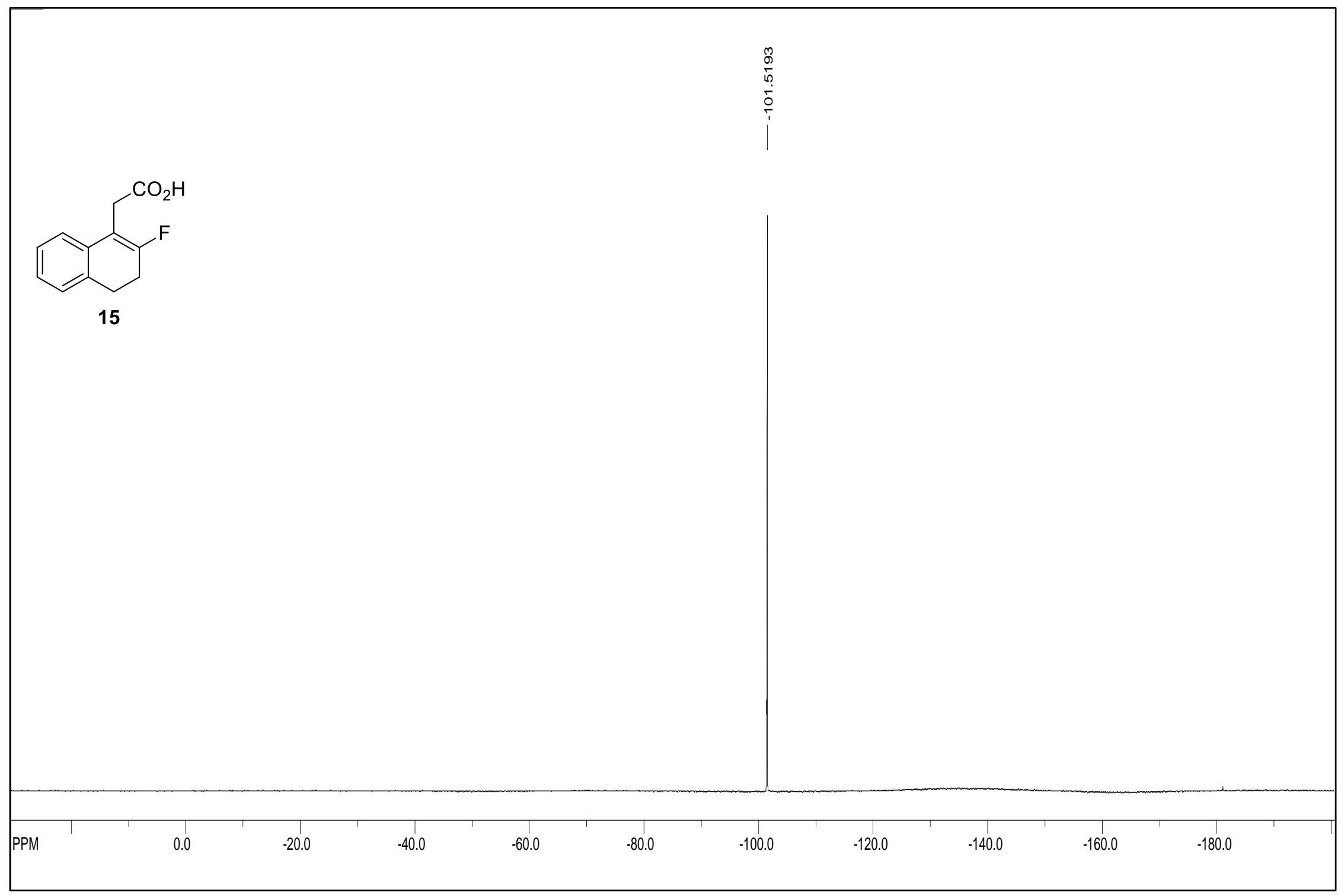




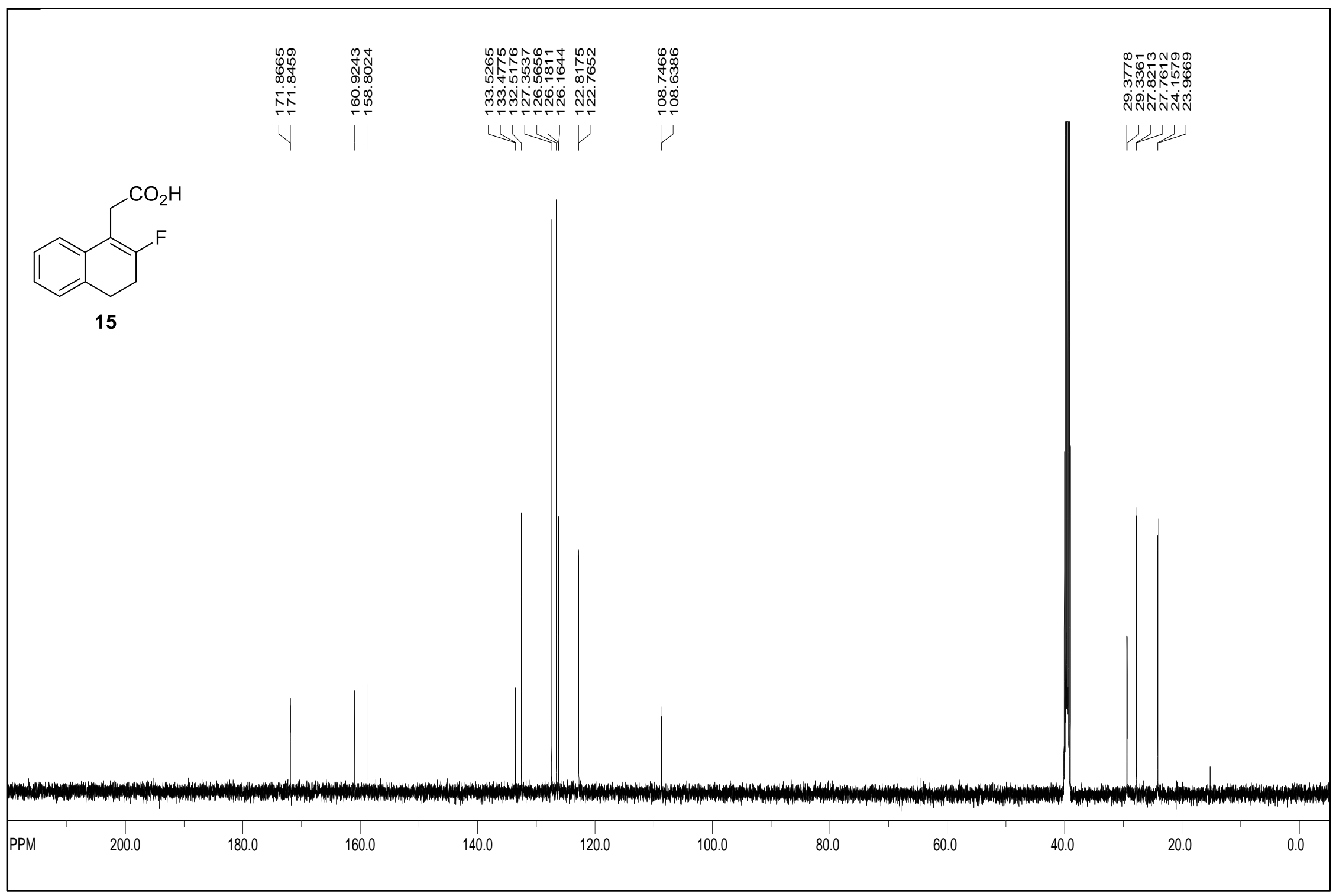




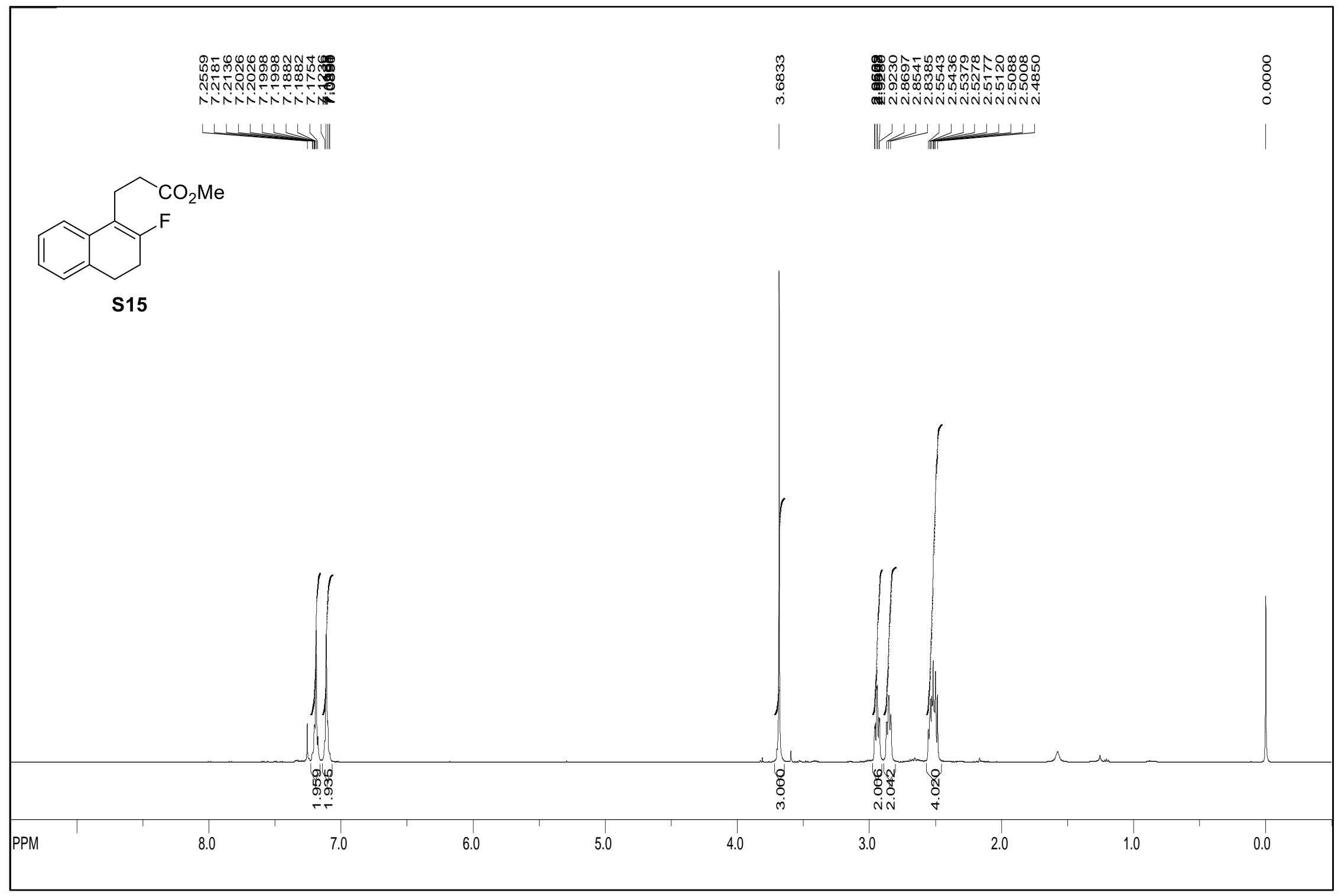




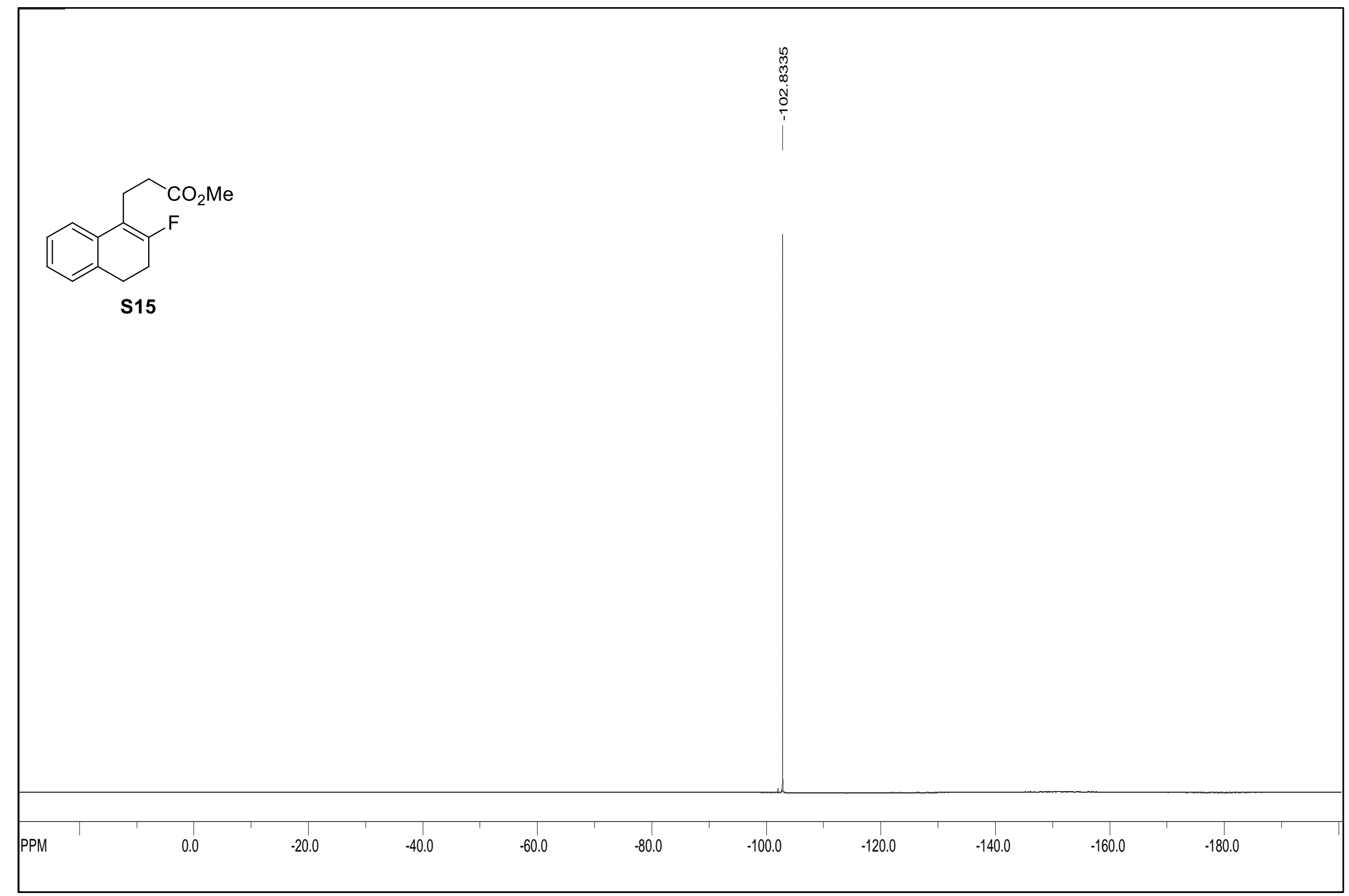




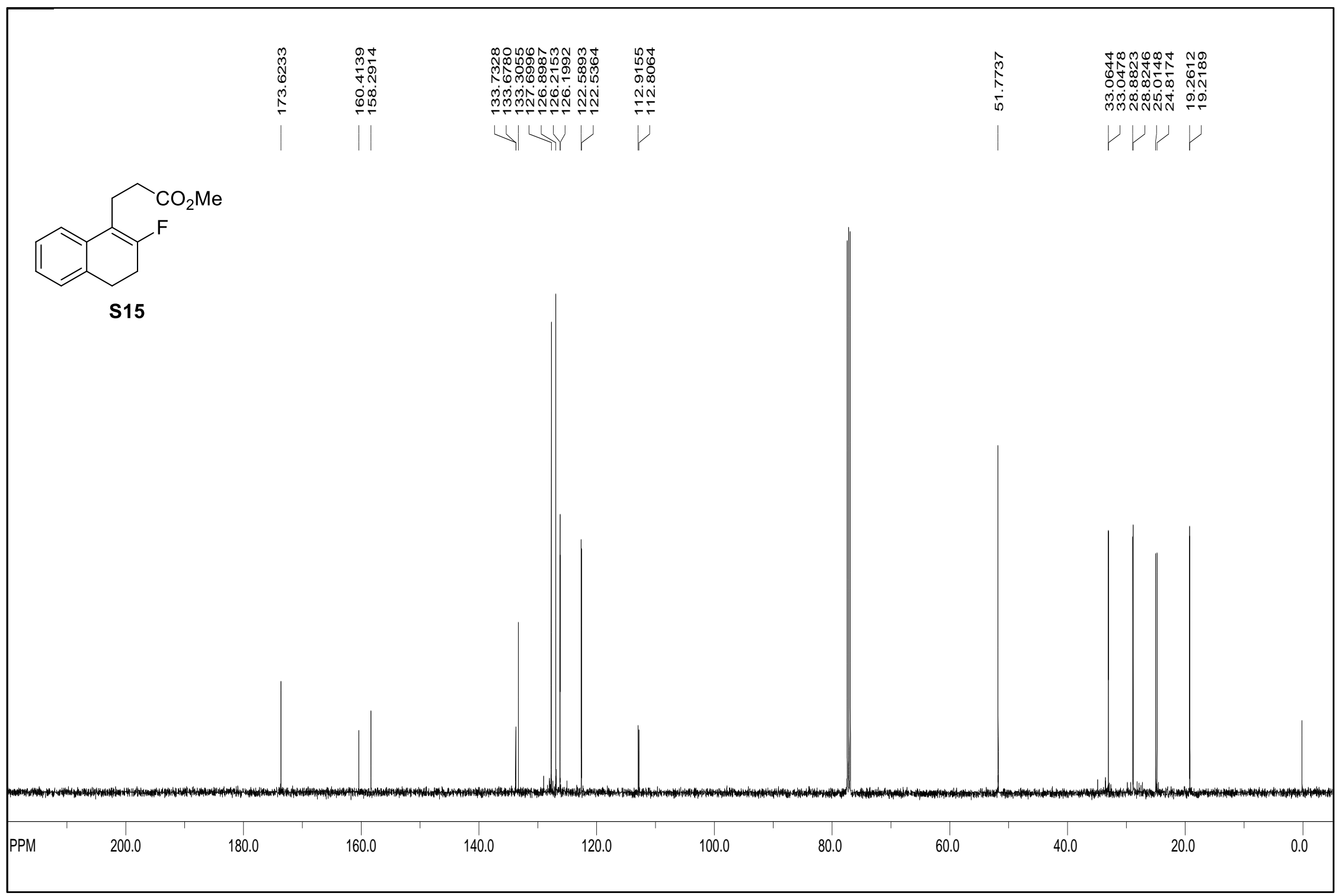




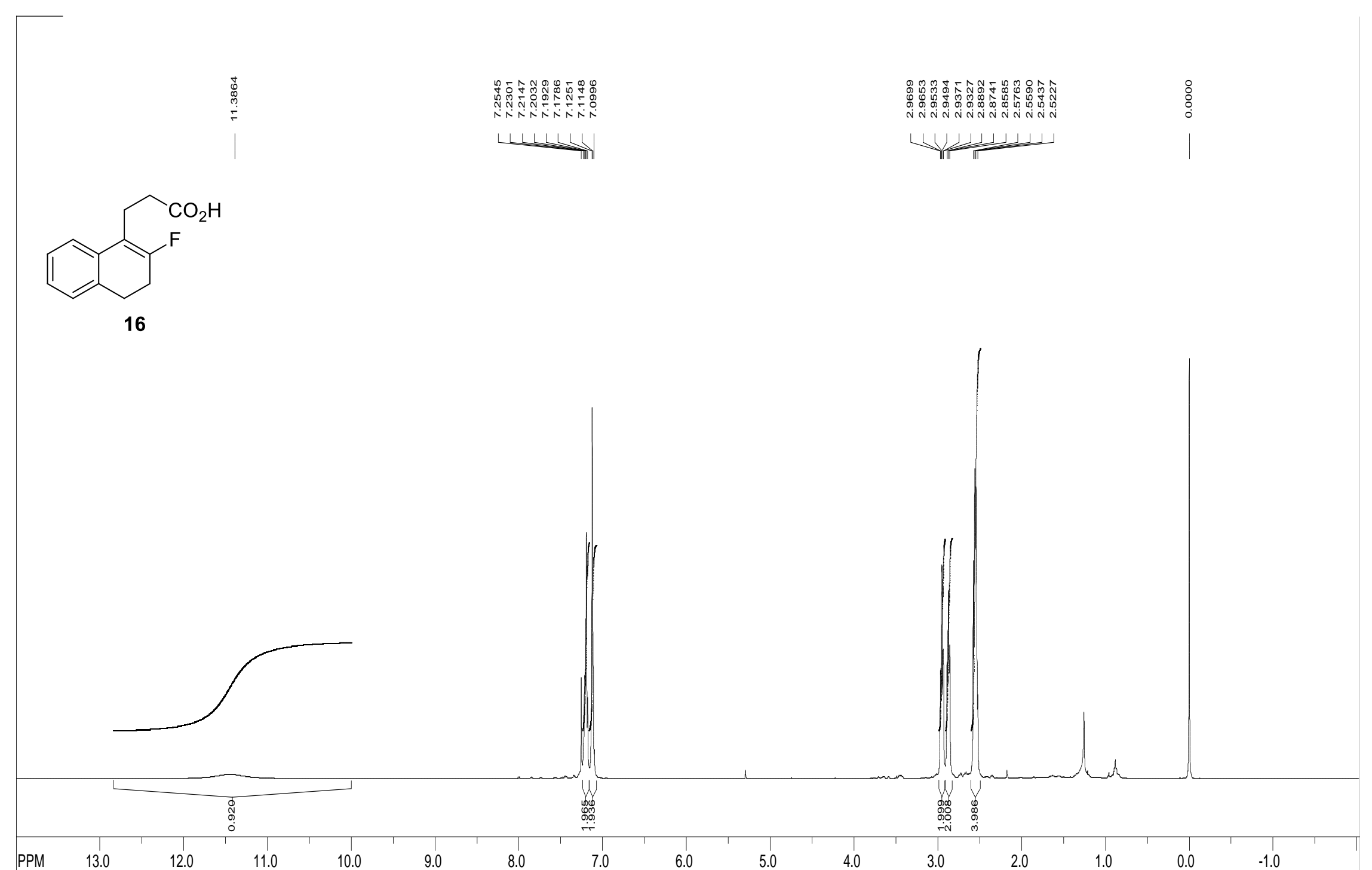




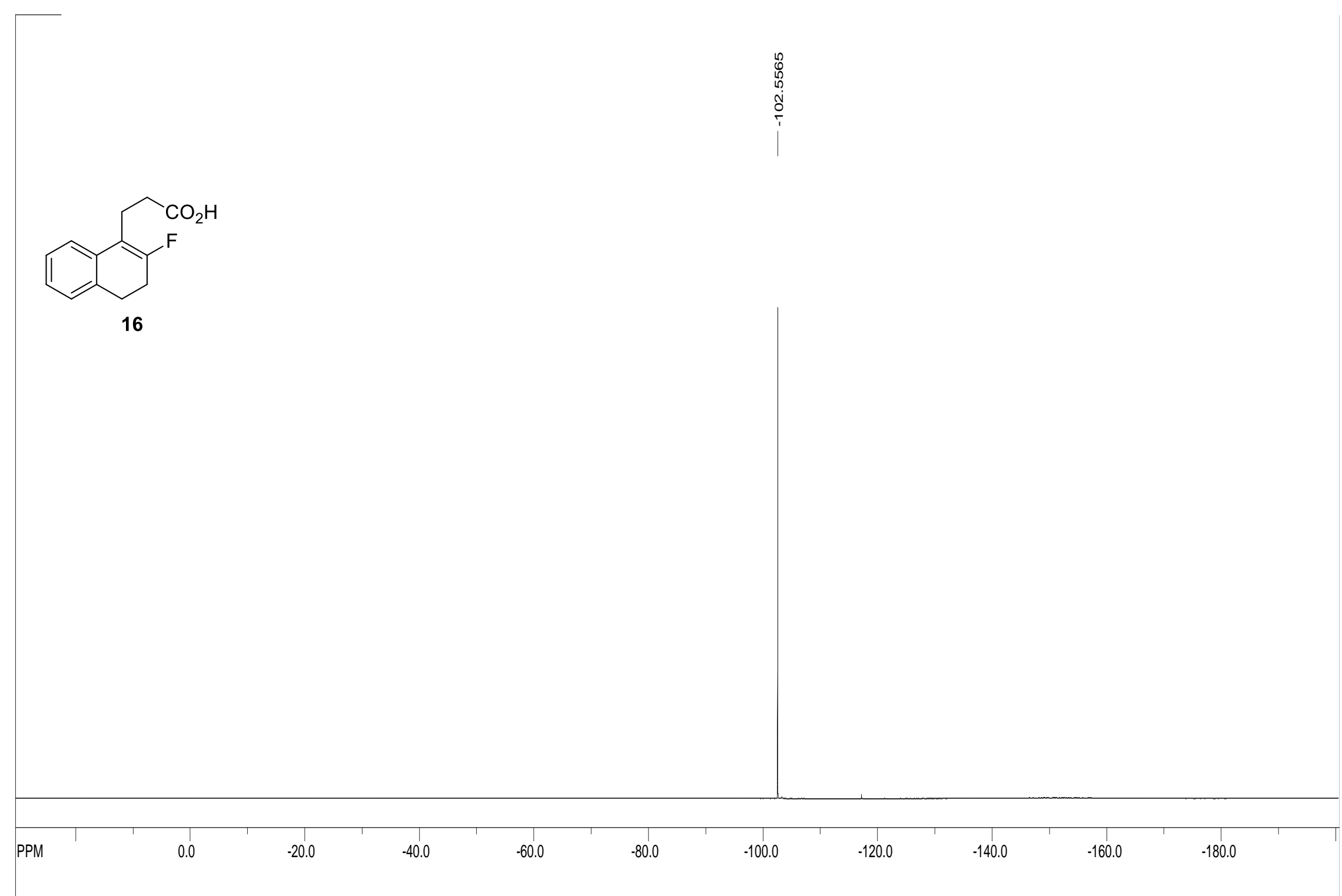




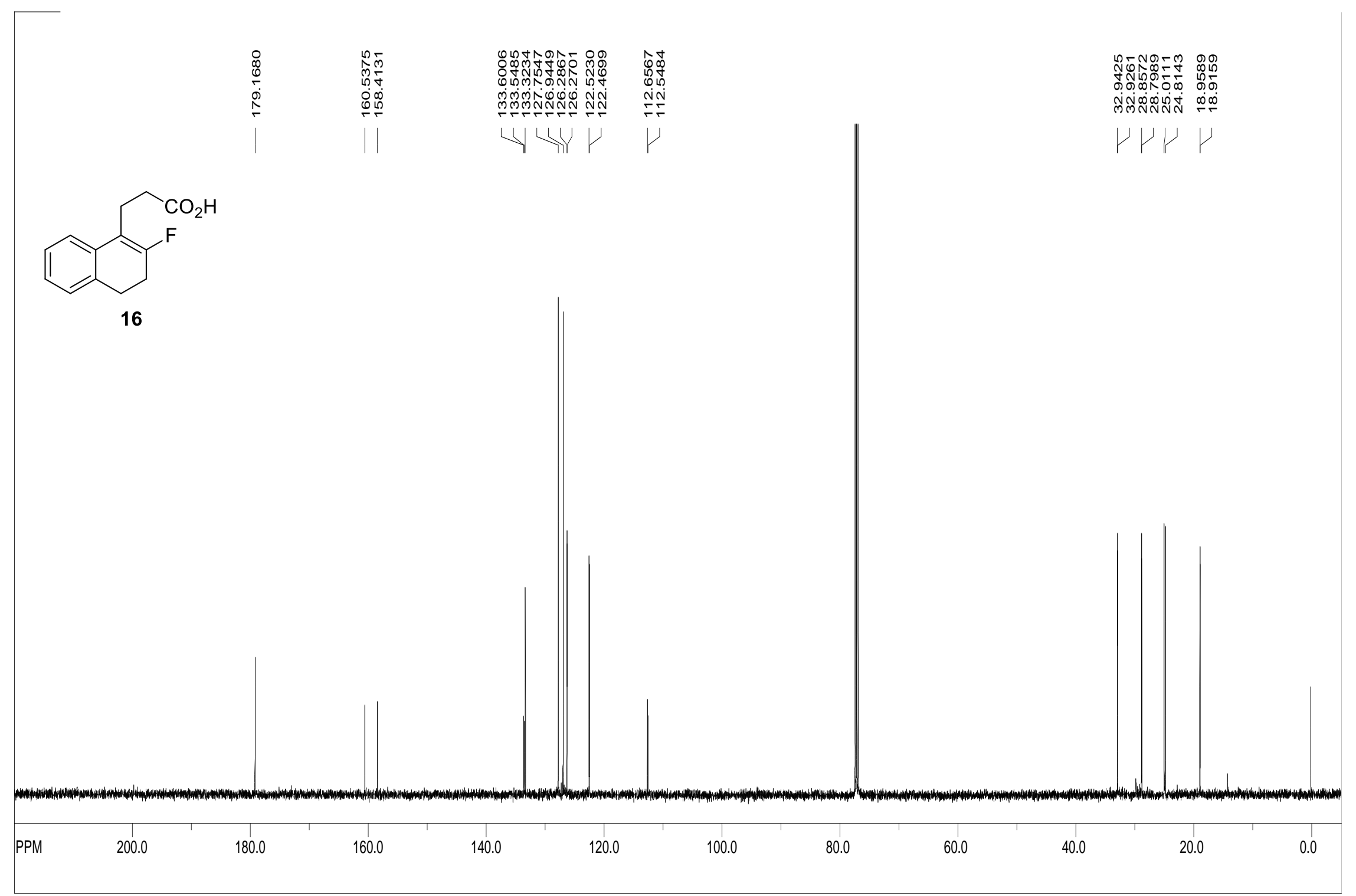




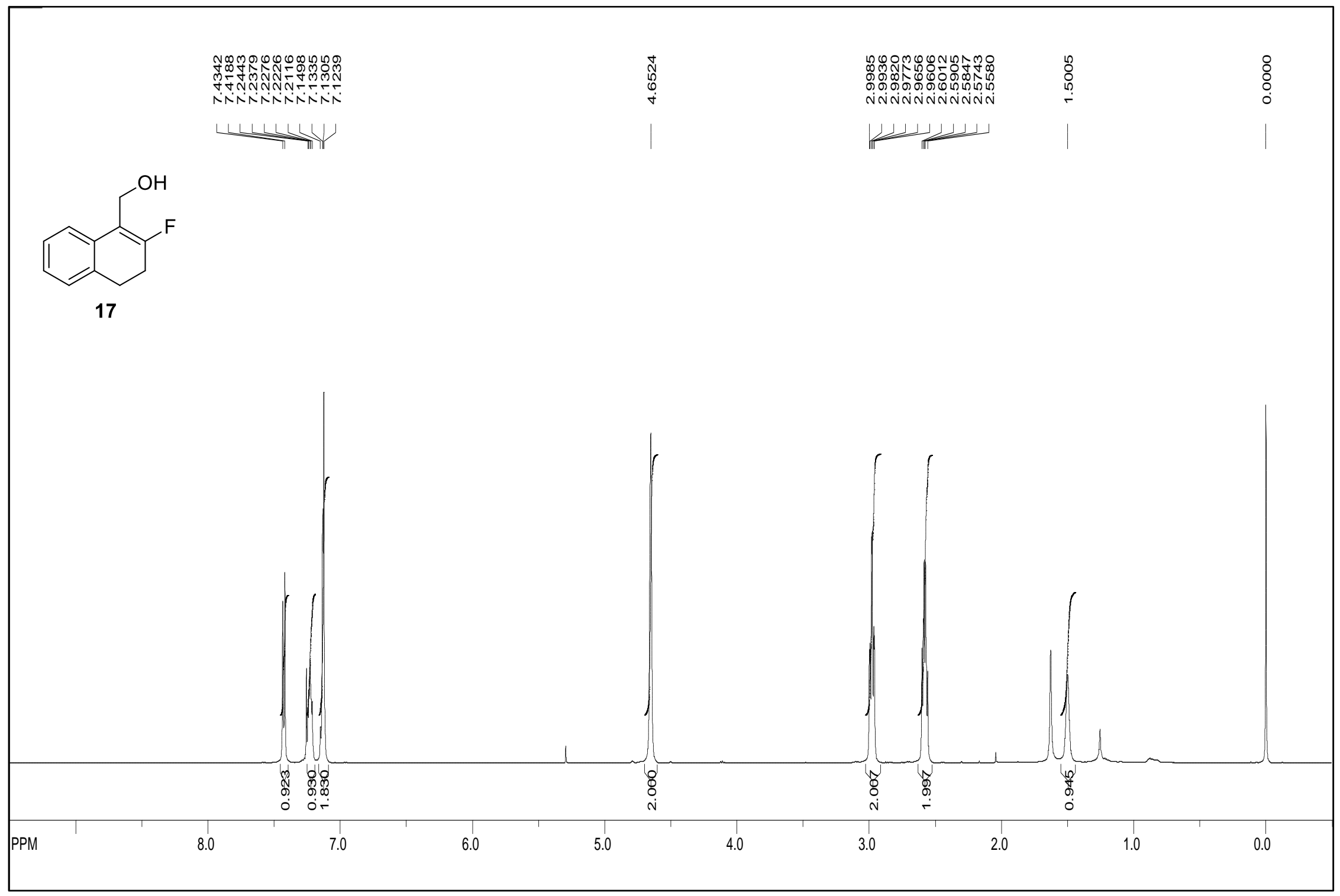




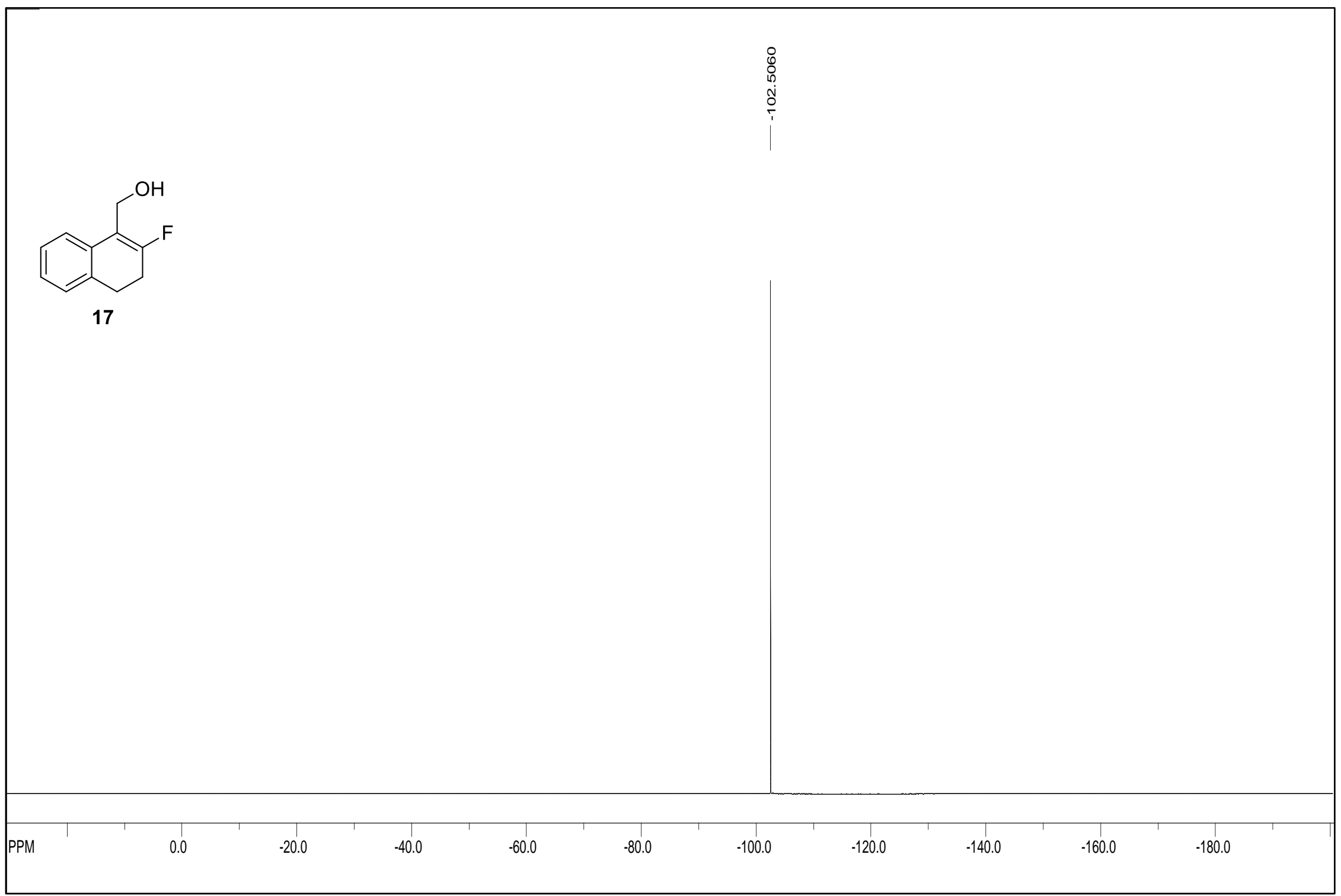




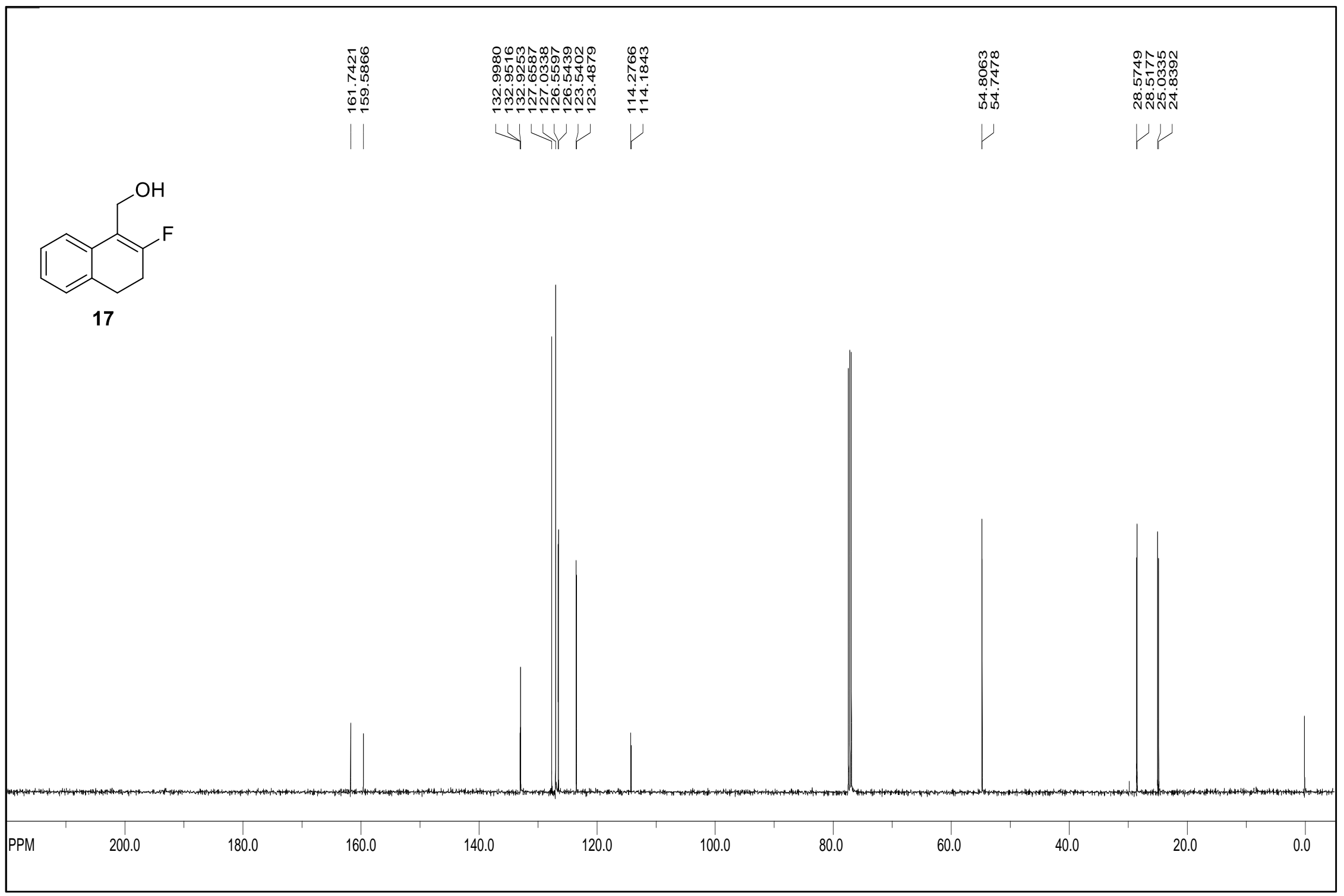




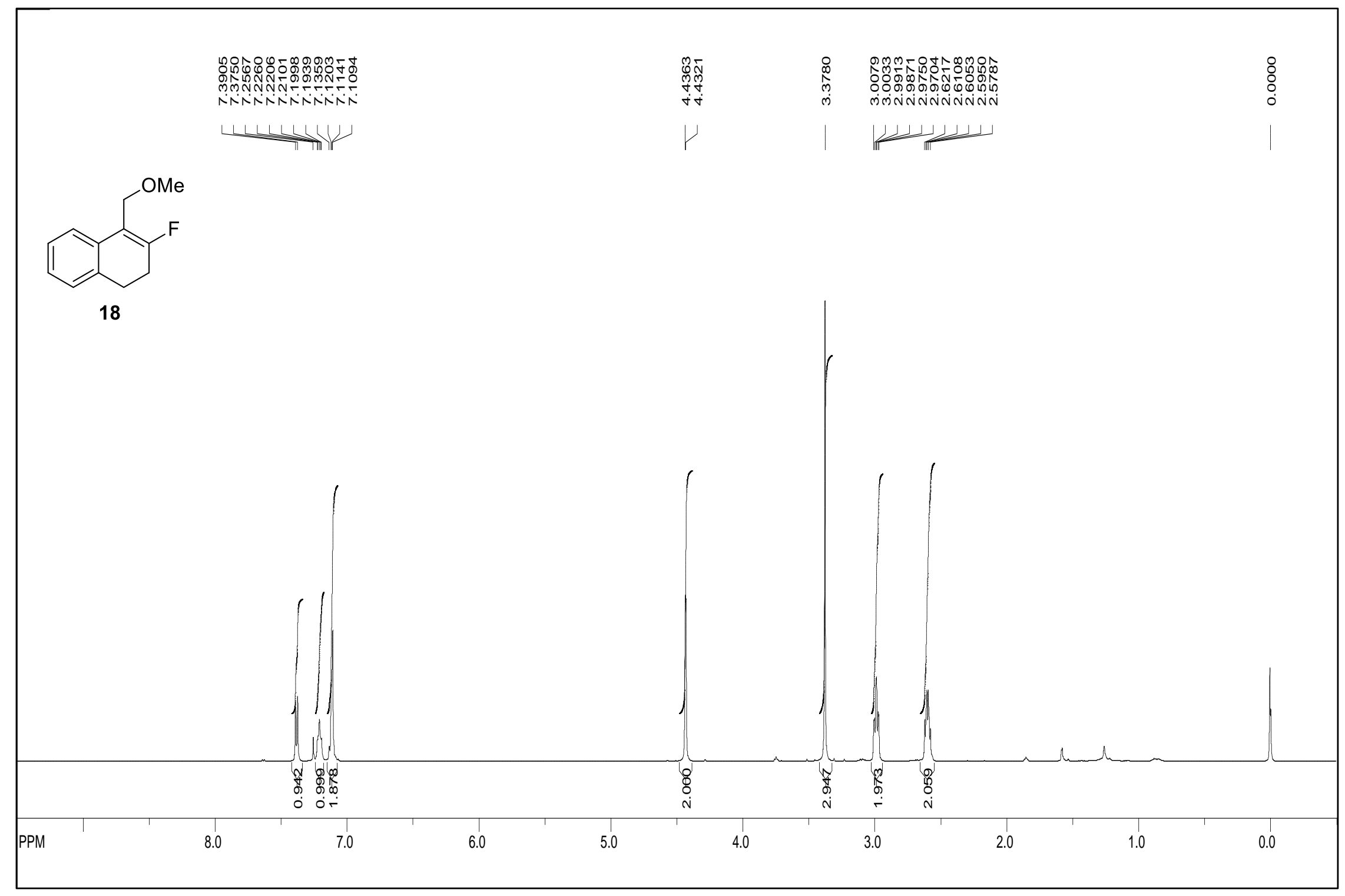


SI-142

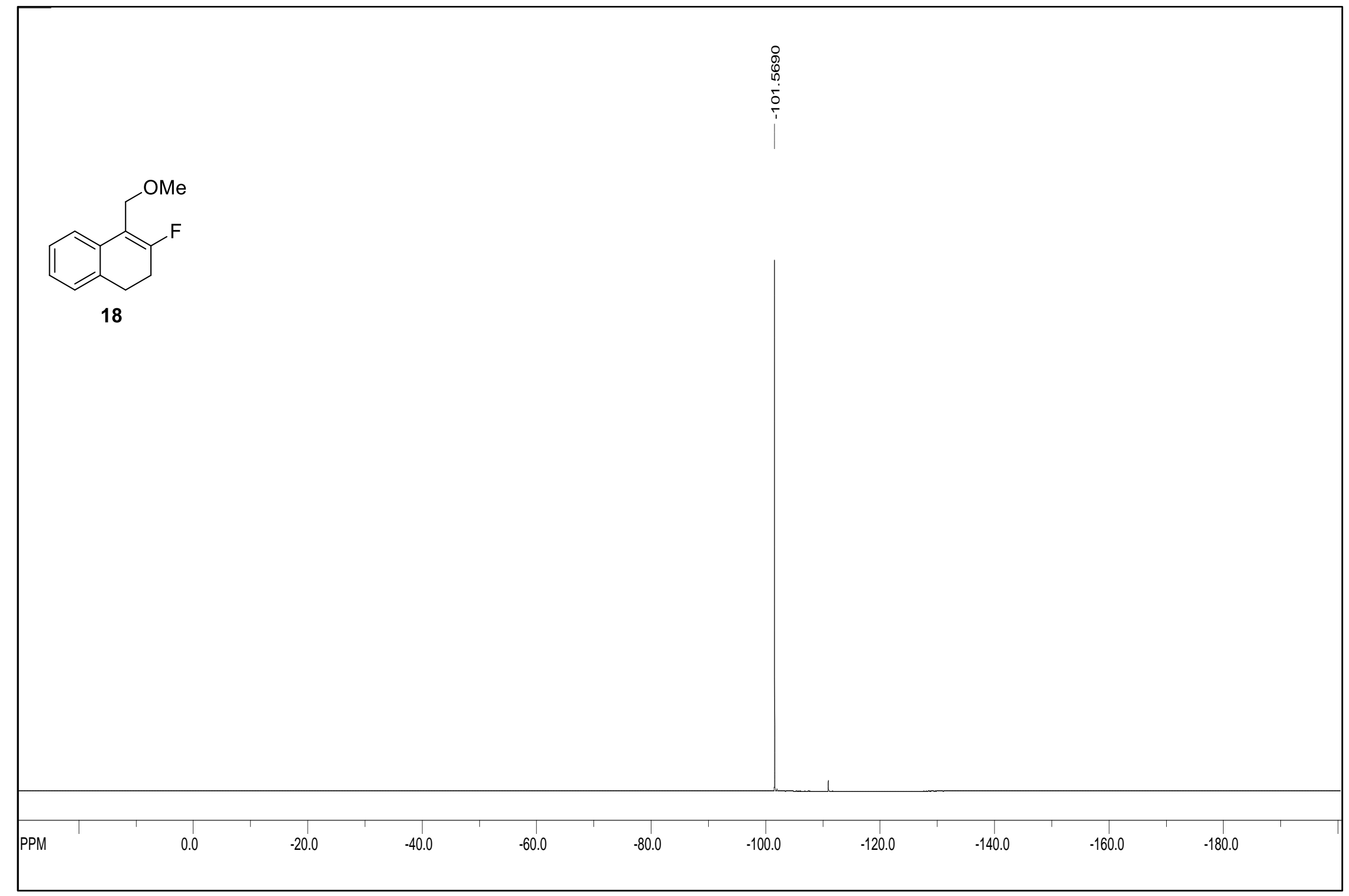




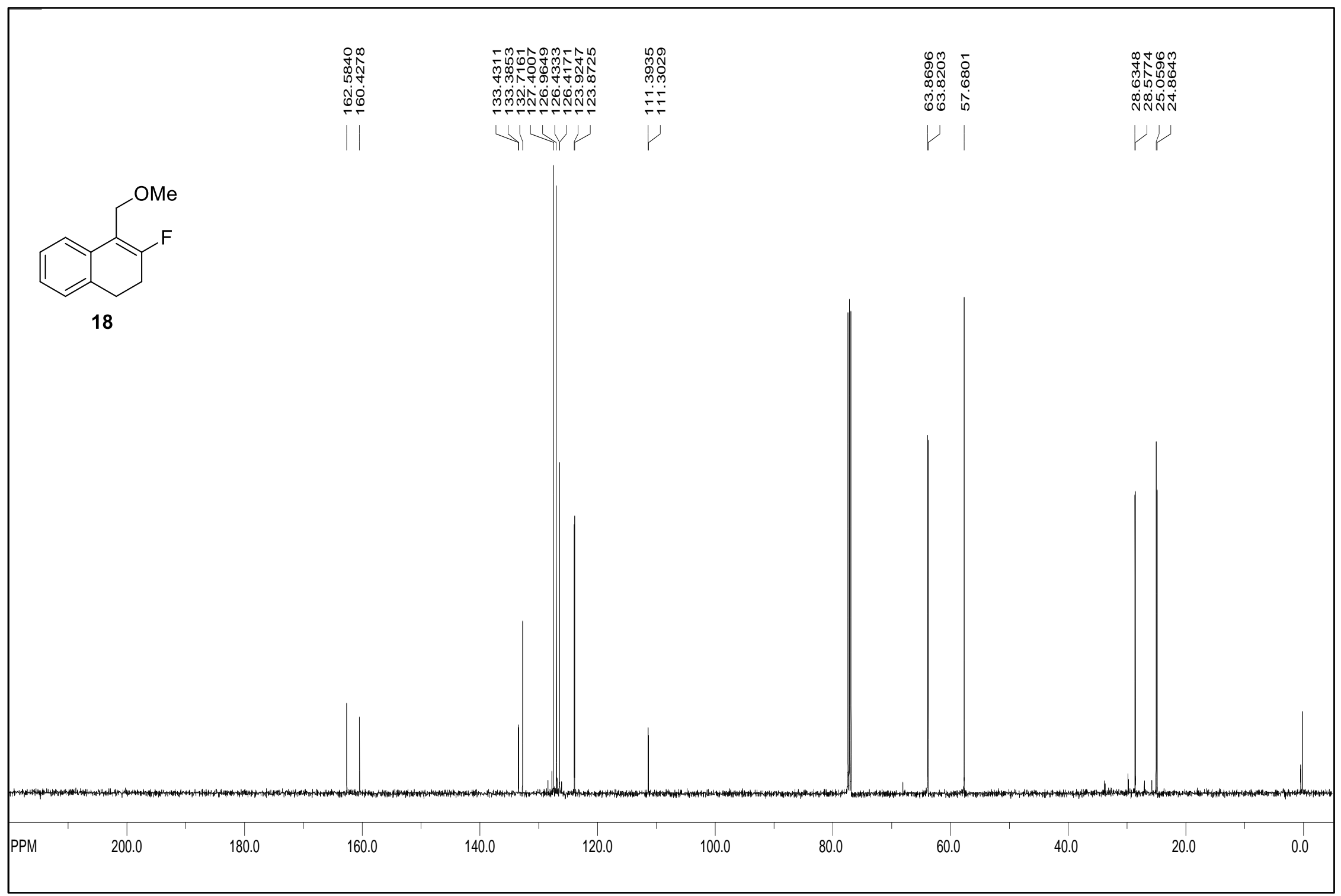




\section{Crystal structure of $(E)-12 a \cdot H C l$}

Procedure for the obtention of a crystal: A sample of $(\boldsymbol{E})-\mathbf{1 2 a}$ was diluted in $\mathrm{Et}_{2} \mathrm{O}$, and $\mathrm{HCl}$ (4 $\mathrm{M}$ solution in 1,4-dioxane) was next added at ambient temperature, resulting in the immediate precipitation of the ammonium salt as a white solid. The solvent and excess reagent were removed in vacuo, and the resulting solid was transferred into a screw-cap vial and dissolved in a minimal amount of EtOAc. The vial was put in the dark at ambient temperature with the cap partially unscrewed to allow slow evaporation of the solvent. After a month, several colorless crystals of $(\boldsymbol{E}) \mathbf{- 1 2 a} \cdot \mathbf{H C l}$ suitable for XRD analysis were obtained.

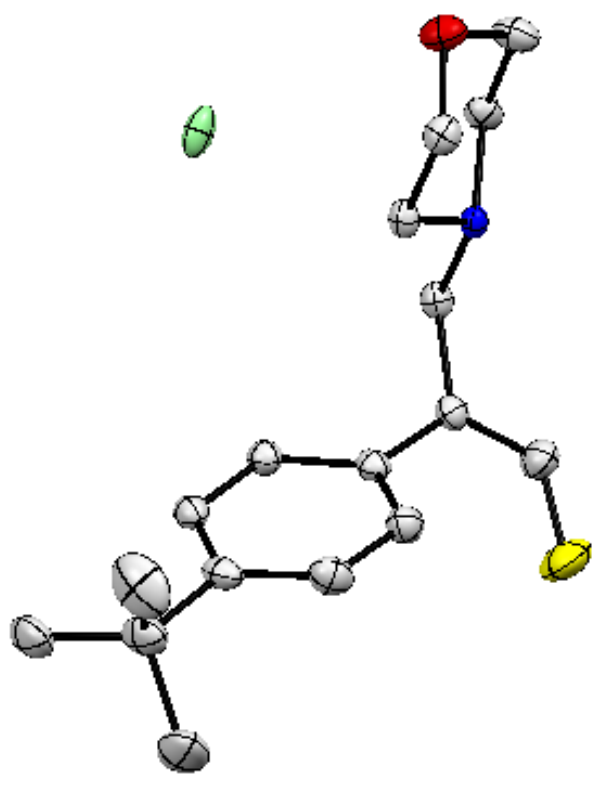

Figure 1. ORTEP diagram of $(\boldsymbol{E})-\mathbf{1 2 a} \cdot \mathbf{H C l}$. Thermal ellipsoids depicted at $50 \%$ probability. Carbon (grey), nitrogen (blue), oxygen (red), fluorine (yellow), and chlorine (green) atoms are shown; hydrogen atoms are omitted for clarity. 